\title{
Sedentary behavior and cardio-metabolic health
}

Citation for published version (APA):

van der Berg, J. D. (2016). Sedentary behavior and cardio-metabolic health: a study into the hazards of sitting too much. [Doctoral Thesis, Maastricht University]. https://doi.org/10.26481/dis.20160706jb

Document status and date:

Published: 01/01/2016

DOI:

10.26481/dis.20160706jb

Document Version:

Publisher's PDF, also known as Version of record

\section{Please check the document version of this publication:}

- A submitted manuscript is the version of the article upon submission and before peer-review. There can be important differences between the submitted version and the official published version of record.

People interested in the research are advised to contact the author for the final version of the publication, or visit the DOI to the publisher's website.

- The final author version and the galley proof are versions of the publication after peer review.

- The final published version features the final layout of the paper including the volume, issue and page numbers.

Link to publication

\footnotetext{
General rights rights.

- You may freely distribute the URL identifying the publication in the public portal. please follow below link for the End User Agreement:

www.umlib.nl/taverne-license

Take down policy

If you believe that this document breaches copyright please contact us at:

repository@maastrichtuniversity.nl

providing details and we will investigate your claim.
}

Copyright and moral rights for the publications made accessible in the public portal are retained by the authors and/or other copyright owners and it is a condition of accessing publications that users recognise and abide by the legal requirements associated with these

- Users may download and print one copy of any publication from the public portal for the purpose of private study or research.

- You may not further distribute the material or use it for any profit-making activity or commercial gain

If the publication is distributed under the terms of Article $25 \mathrm{fa}$ of the Dutch Copyright Act, indicated by the "Taverne" license above, 


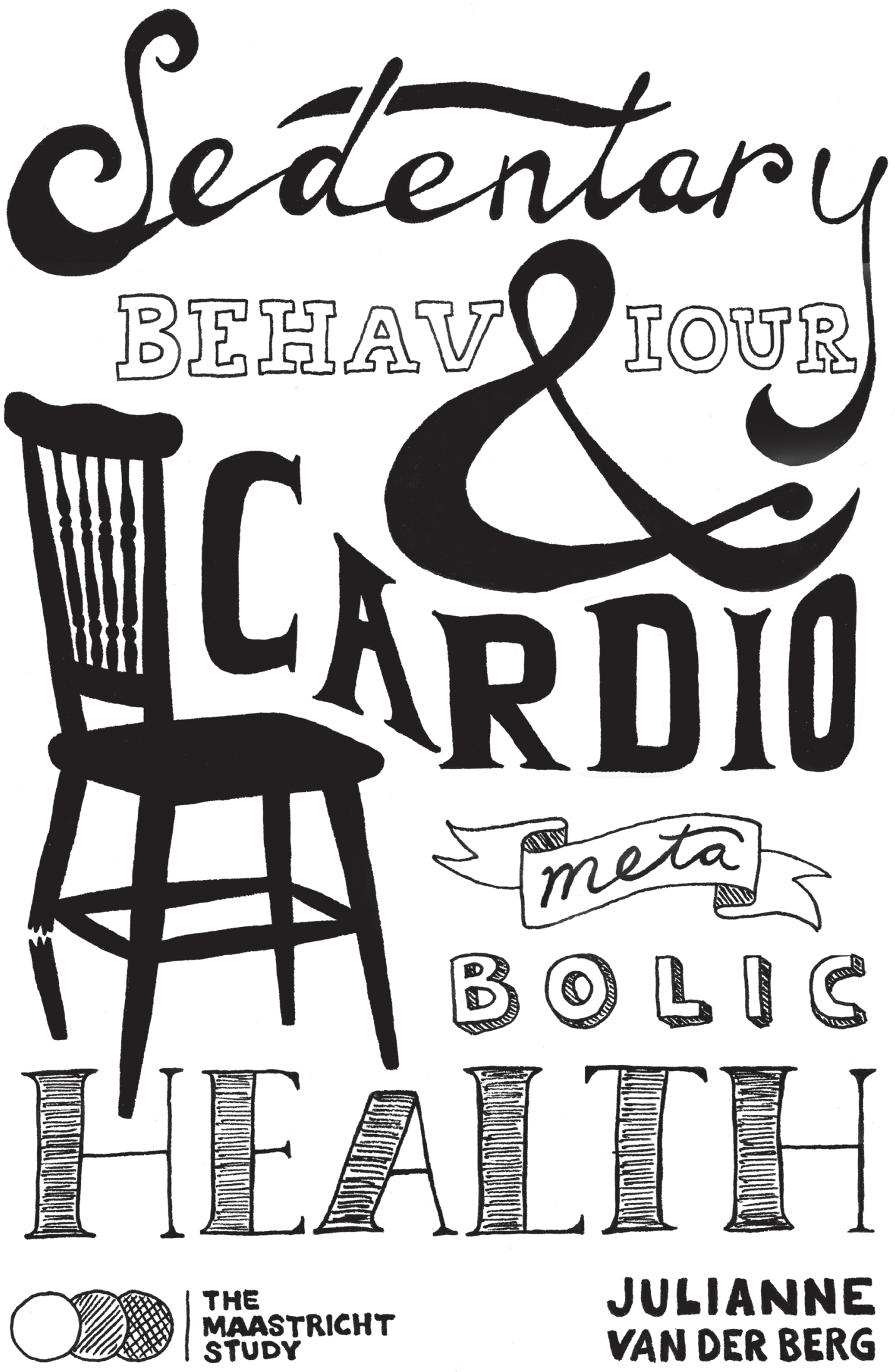




\section{Sedentary behaviour and cardio-metabolic health}

A study into the hazards of sitting too much

Julianne van der Berg 
(C) Julianne van der Berg, Renkum, 2016

Layout: Esther Ris, proefschriftomslag.nl

Cover: $\quad$ Esther Ris, proefschriftomslag.nl

Production: Gildeprint B.V.

ISBN: $\quad$ 978-94-92332-11-0 


\title{
Sedentary behaviour and cardio-metabolic health
}

\author{
A study into the hazards of sitting too much
}

\section{PROEFSCHRIFT}

ter verkriiging van de graad van doctor aan de Universiteit Maastricht, op gezag van de Rector Magnificus, prof. dr. L.L.G. Stoete, volgens het besluit van het College van Decanen, in het openbaar te verdedigen op woensdag 6 juli 2016 om 10.00 uur

door

Julianne Duifje van der Berg 


\section{Promotores:}

Prof. dr. C.D.A. Stehouwer

Prof. dr. H. Bosma

\section{Copromotor:}

Dr. ir. A. Koster

\section{Beoordelingscommissie:}

Prof. dr. M.P.A. Zeegers (voorzitter)

Dr. M. Kars

Prof. dr. L.J.C. van Loon

Prof. dr. M.G.A.A.M. Nijpels (VU medisch centrum)

Dr. H.P. van der Ploeg (VU medisch centrum)

The research presented in this thesis was conducted at the School for Public Health and Primary Care: CAPHRI, Department of Social Medicine, of Maastricht University. CAPHRI participates in the Netherlands School of Primary Care Research CaRe. 


\section{$\underline{\text { TABLE OF CONTENTS }}$}

CHAPTER 1

General introduction

CHAPTER 2

Identifying waking time in $24 \mathrm{~h}$ accelerometry data in adults

using an automated algorithm

CHAPTER 3 .

Measuring back-to-front and side-to-side movement during sedentary activities using a hip-worn tri-axial accelerometer

CHAPTER 4

Midlife determinants associated with sedentary behaviour in old age

CHAPTER 5 .

'Active sedentariness' is associated with cardio-metabolic outcomes

and the metabolic syndrome: The AGES-Reykjavik Study

CHAPTER 6 .

Theoretical effects of replacing sedentary time with standing or stepping

on cardio-metabolic outcomes and type 2 diabetes: The Maastricht Study

CHAPTER 7

Associations of total amount and patterns of sedentary behaviour

with type 2 diabetes and the metabolic syndrome: The Maastricht Study

CHAPTER 8

Associations of total amount and patterns of physical activity

and sedentary behaviour with aortic pulse wave velocity: The Maastricht Study

CHAPTER 9

Associations of total amount and patterns of physical activity and sedentary behaviour with estimated glomerular filtration rate and albuminuria: The Maastricht Study

CHAPTER 10

General discussion

Summary.

Samenvatting....

Valorisation addendum

Dankwoord 
Chapt

照 
General introduction 
Type 2 diabetes mellitus (T2DM) is a chronic, metabolic disease that is characterized by elevated blood glucose levels, which result from defects in insulin action, deficits in insulin secretion, or both. In the long-term, these may lead to the development of diabetes complications such as nephropathy, retinopathy, and neuropathy. In addition, having T2DM increases the risk for cardiovascular disease dramatically, due to the increased occurrence of conditions such as adiposity, dyslipidaemia, and a high blood pressure. ${ }^{1}$

To date, diabetes in all its forms, is one of the most prevalent diseases worldwide. A general estimation is that 415 million individuals are living with this disease, and this number is expected to rise to 642 million in 2040, representing 10\% of the total adult population. ${ }^{2}$ The high prevalence of T2DM $187-91 \%$ of all people with diabetes are estimated to have $\mathrm{T}^{2} \mathrm{DM}^{2}$ ) and its complications and comorbidities impose an enormous burden on patients, healthcare systems, and society, so preventing T2DM is necessary.

For prevention strategies, it is important to focus on targets that 1) are related to factors which play a role in the development of T2DM, its complications and comorbidities such as the metabolic syndrome and dysfunction of micro- or macrovascular systems, and 2) are modifiable and amenable for intervention, for example physical activity.

\section{Physical activity \& sedentary behaviour:}

\section{Too much sitting differs from too little exercise}

From ancient times physical activity has been studied in relation to disease development. Early beliefs about physical activity and health were written 5,000 years ago in India and China, and also ancient Greek physicians including Hippocrates and Galen, prescribed exercise to prevent disease. ${ }^{3}$ The modern era of physical activity epidemiology started in 1953 with The London Bus Study of Jeremy Morris, ${ }^{4}$ who examined whether bus conductors (who walked and climbed stairs in the bus all day to collect tickets) were at lower risk for cardiovascular events than bus drivers (who spent the majority of their day sitting). Indeed, the conductors had a lower incidence of coronary heart-disease than the drivers. ${ }^{4}$ Since then the body of evidence linking physical activity to health has been growing. For example, insufficient levels of physical activity have been associated with T2DM, cardiovascular disease, cancer and even premature mortality. ${ }^{5-8}$ So not surprisingly, public health organisations and governmental health departments have developed recommendations for the daily amount of physical activity that should be attained to remain healthy and prevent disease. 6,9 
Nevertheless, physical activity, especially at a high intensity level (e.g. exercise), is only a small part of all daily activity, even if physical activity guidelines are met (i.e. 30 $\mathrm{min} /$ day). So typically, daytime is spent in regular activities such as walking, standing, and sitting (i.e. sedentary behaviour). Sedentary behaviour is defined as any waking behaviour that is characterized by a low energy expenditure $(\leq 1.5$ metabolic equivalents (METs)) while in a sitting or reclining position, such as watching TV, using the computer or driving. ${ }^{10}$ For most individuals, the majority of their waking hours, up to $80 \%$, is spent in sedentary positions, ${ }^{11-14}$ but it was not until the early 2000 s that researchers started to focus on sedentary behaviour as a risk factor for health. In 2001, Hu et al. were the first to report on effects of sedentary behaviour on type 2 diabetes. ${ }^{15}$ Thereupon, several studies focused on sedentary behaviour, and reported associations between large amounts of sedentary time and detrimental health outcomes including the metabolic syndrome, ${ }^{16-19}$ T2DM, ${ }^{20,21}$ cardiovascular disease, ${ }^{20-22}$ and premature mortality. ${ }^{20,21,23}$ Importantly, since these associations were independent of the amount of high intensity physical activity, sedentary behaviour cannot be viewed as the lack of high intensity physical activity, but should be viewed as a distinct health behaviour, with its own nature and physiology. ${ }^{24-27}$

\section{Measuring sedentary behaviour: Accelerometry}

In early sedentary behaviour studies, measurement of amount of sedentary time was based on self-reporting methods, such as questionnaires. Frequently used questionnaires focus on specific types of sedentary behaviour, most often watching TV. Although TV viewing time can be seen as a relevant part of total daily sedentary time, it is not representative for total sedentary time because other types of sedentary behaviour, for example using the computer or transportation by car, bus or train, should be taken into account as well. ${ }^{28,29}$ Furthermore, questionnaires and other self-reporting methods such as diaries, are limited due to issues of recall and reporting bias. ${ }^{28,29}$

The introduction of accelerometry in sedentary behaviour studies during the late 2000 s provided researchers a measurement tool which overcomes limitations of self-reporting methods. Accelerometers are small, lightweight, portable devices that provide information on the frequency, duration and intensity of activity of an individual. ${ }^{28,30}$ The ActiGraph accelerometer (Figure 1.1) is a commonly used device that is usually worn on

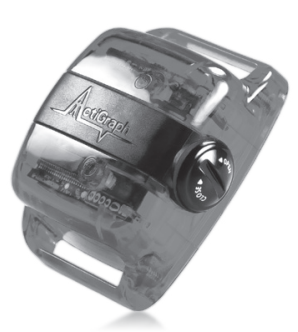

Figure 1.1: ActiGraph GT3X accelerometer. Dimensions: $4.4 \times 3.3 \times$ $1.5 \mathrm{~cm}$. Weight: $19 \mathrm{~g}$. 
the waist or hip for the duration of one week. The device measures motion (acceleration) of the body segment to which it is attached (e.g. waist or hip), usually in the vertical (up-and-down) direction. To determine activity levels, the raw acceleration data are converted into 'counts'. These counts are summed for a specific time period, usually a minute, and these 'counts per minute' (cpm) are used to classify activity; more cpm represents higher intensity activity. For each type of activity, ranging from sedentary behaviour to high intensity physical activity, specific cut-off points have been determined. ${ }^{31}$ Since sedentary behaviour is characterized by low intensity levels, $<100 \mathrm{cpm}$ is usually used to identify sedentary time. ${ }^{31}$ In contrast, moderate to vigorous physical activity (MVPA), such as volleyball or running, ${ }^{32}$ is characterized by high intensity activity levels and identified when $\geq 2,020 \mathrm{cpm}$ are recorded. ${ }^{33}$

\section{Technological developments: New information on sedentary behaviour}

Due to technological developments in the last few years, sedentary behaviour measurement has improved significantly. The first generation of accelerometers measured acceleration in the up-and-down direction, but in time accelerometers became available that recorded data in three directions, i.e. vertical (up-and-down), anteroposterior (back-to-front), and mediolateral (side-to-side). Therefore, tri-axial accelerometers can provide a more comprehensive measurement of activity, in comparison with uni-axial devices. ${ }^{30}$

Sedentary behaviour measurement has been improved further by the introduction of the activPAL physical activity monitor, because this device measures both acceleration and posture (Figure 1.2). The activPAL accelerometer is a small $(5.3 \times 3.5 \times$ $0.7 \mathrm{~cm})$, lightweight $(15 \mathrm{~g})$ tri-axial accelerometer that should be attached, after it has been waterproofed, directly to the skin on the front of the thigh. Thus, it can detect a sitting/lying posture (thigh horizontally)

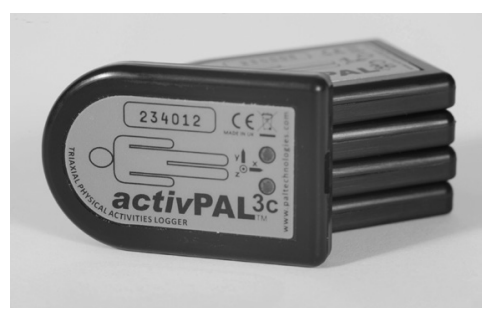

Figure 1.2: activPAL physical activity monitor. Dimensions: $5.3 \times 3.5 \times 0.7$ $\mathrm{cm}$. Weight: $15 \mathrm{~g}$. versus an upright posture (thigh vertically). ${ }^{34}$ The assessment of sedentary time using this technology has shown to be more accurate than acceleration-based assessment of sedentary time, because when using only acceleration data, upright positions can be misclassified as sedentary time when activity counts are low. ${ }^{35,36}$ In addition, a complete assessment of all daily activity (24 hour per day on multiple days) has become feasible due to the waterproof attachment and the small dimensions of the activPAL accelerometer. 
Next to the technological developments of the accelerometer, its data processing and analysing techniques have improved. Until recently, the construct used to quantify sedentary behaviour was the total amount of sedentary time per day. However, since not all sedentary behaviour is bad, and even certain amounts are needed for rest and recovery, the pattern of sedentary behaviour should be taken into consideration, i.e. how sedentary time is accumulated. For example, multiple short sedentary periods versus one prolonged period. Constructs to quantify these patterns are sedentary breaks, sedentary bouts, and sedentary bout duration (Figure 1.3). A sedentary break is an interruption of sedentary time, so each transition from a sedentary to an upright position. A sedentary bout is a sedentary period without interruption, which can have any duration. The average sedentary bout duration is calculated by dividing total sedentary time by the total number of sedentary bouts. The relevance of sedentary behaviour patterns has been indicated in studies that demonstrated associations between constructs of sedentary behaviour patterns and metabolic health. ${ }^{37-40}$

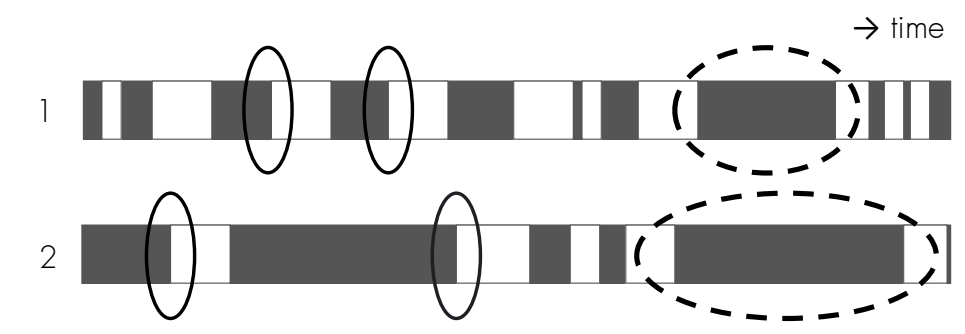

Figure 1.3: Two examples of sedentary behaviour patterns. Dark bars indicate sedentary time; light bars indicate non-sedentary time. Solid lines represent sedentary breaks (interruptions of sedentary time); dashed lines represent sedentary bouts (uninterrupted periods of sedentary time).

\section{This dissertation: Expanding the sedentary behaviour research area}

Previous studies on sedentary behaviour have demonstrated that large amounts of sedentary time are associated with several detrimental cardio-metabolic outcomes such as adiposity, ${ }^{16-19}$ dyslipidaemia, ${ }^{16-19}$ high blood glucose levels, ${ }^{16-19,41,42}$ the metabolic syndrome, ${ }^{16,17,19}$ and T2DM. 15,20,21,42 However, most of these studies used self-reporting methods to estimate total sedentary time, and patterns of sedentary time were not examined. Due to the limitations of self-reporting measures and limited information on sedentary behaviour patterns, it is necessary to conduct studies that use accelerometry to objectively measure sedentary behaviour and its patterns. These studies can provide 
insight in the role of sedentary behaviour in cardio-metabolic health and may therefore contribute to the development of strategies aiming to prevent T2DM, its complications and comorbidities.

This dissertation includes accelerometry studies that focussed on several constructs of sedentary behaviour and their associations with cardio-metabolic outcomes. The studies were conducted in two cohorts using two types of accelerometers: the activPAL3 physical activity monitor (PAL Technologies, Glasgow, UK) in The Maastricht Study and the ActiGraph GT3X accelerometer (ActiGraph, Fort Walton Beach, FL, USA) in the AGES-Reykjavik Study. The activPAL accelerometer was used for the 24 hour per day posture-based measurement of both the amount and pattern of daily activity. Although 24 hour measurement provides complete assessment of total daily activity levels, a method for identifying waking time in the 24 hour period is necessary in order to correctly determine the amount of sedentary time and physical activity accumulated during the waking period. Previously used methods such as manually determining waking hours or using diaries, are not feasible in studies with a large sample size due to the workload for participants and researchers. Since the activPAL accelerometer has not been used previously in a large-scale study, a method to automatically determine wake and bed times on an individual level (i.e. different wake and bed times on each day for each participant) had to be developed (chapter 2).

Also, tri-axial data of the ActiGraph accelerometer has not been used before despite the fact that a more comprehensive measurement of sedentary behaviour can be obtained compared to uni-axial data. To date, sedentary time is usually based on cpm in the vertical axis only (i.e. $<100 \mathrm{cpm}$ ). ${ }^{31}$ Although sedentary time is characterized by low or no movement in the vertical (up-and-down) direction, movement with the upper-body in the anteroposterior and mediolateral directions (back-to-front and side-to-side) is likely to occur, for example when doing desk work. However, it was not known whether a hip-worn tri-axial accelerometer can detect these upper-body movements while being in a sedentary position. Therefore, an experimental study had to be conducted to examine whether a hip-worn tri-axial accelerometer is able to measure movement in the anteroposterior and mediolateral axes during sedentary activities (chapter 3).

The following study of this dissertation examined which demographic, socioeconomic, lifestyle and biomedical determinants assessed in midlife were associated with sedentary behaviour in old age, in order to identify individuals at increased risk for a (highly) sedentary lifestyle (chapter 4). Next, three studies on cardio-metabolic outcomes, the metabolic 
syndrome and T2DM were conducted. The first study examined whether being active with the upper-body during sedentary activities ('active sedentariness') was associated with cardio-metabolic outcomes and the metabolic syndrome (chapter 5). The second study examined whether reallocating sedentary time to standing or stepping was associated with cardio-metabolic outcomes, the metabolic syndrome, and T2DM (chapter 6). The third study examined associations of total amount and patterns of sedentary behaviour with the metabolic syndrome and T2DM (chapter 7). Subsequently, two studies on risk factors for cardio-metabolic diseases were conducted; the associations of sedentary behaviour, patterns of sedentary behaviour and physical activity with aortic stiffness (chapter 8) and kidney function (chapter 9) were examined.

\section{Cohort studies used in this dissertation}

- The Maastricht Study

The Maastricht Study ${ }^{43}$ is an ongoing observational, population-based cohort study that focuses on the aetiology, pathophysiology, complications, and comorbidities of T2DM and which is characterised by an extensive phenotyping approach. In this dissertation data is used from the first 3,451 participants who completed the baseline survey between November 2010 and September 2013. It was used to examine 1) associations of sedentary behaviour and physical activity with the metabolic syndrome, T2DM, vascular function and kidney function, and 2) associations of reallocating sedentary time with cardio-metabolic health.

- The AGES-Reykjavik Study

The Age, Gene/Environment Susceptibility (AGES)-Reykjavik Study ${ }^{44}$ originates from the Reykjavik Study, which was established in 1967 and comprised a random sample of 30,795 participants who were residing in Reykjavik, Iceland. In 2002-2006, the AGES-Reykjavik Study took place in 5,764 participants of the original cohort and the follow-up (AGESII-Reykjavik Study) took place in 2007-2011 among 3,411 participants. From April 2009 to June 2010, an accelerometry substudy was performed in 658 participants of this cohort. Data of this substudy was used to examine 1) associations between active sedentariness and cardio-metabolic health and 2) associations between midlife factors and sedentary time in old age. 


\section{Outline of this dissertation}

In chapter 2 we examined the accuracy of a newly developed automatic algorithm to identify waking time in 24 hour accelerometry data.

In chapter 3 we conducted an experimental study to examine the ability of a hip-worn triaxial accelerometer to measure active sedentariness.

In chapter 4 we examined, in the AGES-Reykjavik Study, associations of demographic, socioeconomic, lifestyle, and biomedical determinants measured in midlife with sedentary behaviour in old age.

In chapter 5 we examined, in the AGES-Reykjavik Study, associations of active sedentariness with cardio-metabolic outcomes and the metabolic syndrome.

In chapter 6 we examined, in The Maastricht Study, associations of theoretical reallocations of sedentary time to standing or stepping with cardio-metabolic outcomes, the metabolic syndrome and T2DM.

In chapter 7 we examined, in The Maastricht Study, the associations of the total amount and patterns of sedentary behaviour with the metabolic syndrome and T2DM.

In chapter 8 we examined, in The Maastricht Study, the associations of total amount and patterns of physical activity and sedentary behaviour with aortic stiffness (aortic pulse wave velocity).

In chapter 9 we examined, in The Maastricht Study, the associations of total amount and patterns of physical activity and sedentary behaviour with kidney function (estimated glomerular filtration rate and albuminuria).

In chapter 10, we discussed the main results described in earlier chapters and placed these in a broader context by addressing methodological considerations, evaluating implications for public health and clinical practice, and providing directions future research. 


\section{REFERENCES}

1. Sarwar N, Gao P, Kondapally Seshasai S, Gobin R, Kaptoge S, Di Angelantonio E, et al. Diabetes mellitus, fasting blood glucose concentration, and risk of vascular disease: A collaborative meta-analysis of 102 prospective studies. Lancet. 2010;375(9733):2215-22.

2. International Diabetes Federation. IDF Diabetes Atlas. 2015.

3. Bouchard C, Blair SN, Haskell WL. Physical Activity and Health. 2012. 441 p.

4. Morris JN, Hardy JA, Raffle PAB, Roberts CG, Parks JW. Coronary heart-disease and physical activity of work. Lancet. 1953;262(6796): 1111-20.

5. Lee I-M, Shiroma EJ, Lobelo F, Puska P, Blair SN, Katzmarzyk PT. Effect of physical inactivity on major non-communicable diseases worldwide: An analysis of burden of disease and life expectancy. Lancet. 2012;380(9838):219-29.

6. US Department of Health and Human. 2008 Physical Activity Guidelines for Americans. 2008.

7. World Health Organization. Global recommendations on physical activity for health. 2010.

8. Warburton DE, Charlesworth S, Ivey A, Nettlefold L, Bredin SS. A systematic review of the evidence for Canada's Physical Activity Guidelines for adults. Int J Behav Nutr Phys Act. 2010;7:39.

9. Hildebrandt $\mathrm{H}$, Ooijendijk $M$, Hopman $M$. Trendrapport Bewegen en Gezondheid. TNO Kwaliteit van Leven. 2010.

10. Sedentary Behaviour Research Network. Standardized use of the terms "sedentary" and "sedentary behaviours." Appl Physiol Nutr Metab. 2012;37:540-2.

11. World Health Organization. Global status report on noncommunicable diseases 2014. 2014.

12. Matthews $C E$, Chen KY, Freedson PS, Buchowski MS, Beech M, Pate RR, et al. Amount of time spent in sedentary behaviors in the United States, 2003-2004. Am J Epidemiol. 2008; 167(7):875-81.
13. Davis MG, Fox KR, Hillsdon M, Sharp DJ, Coulson JC, Thompson JL. Objectively measured physical activity in a diverse sample of older urban UK adults. Med Sci Sports Exerc. 201 1;43(4):647-54.

14. Arnardottir NY, Koster A, Van Domelen DR, Brychta RJ, Caserotti P, Eiriksdottir G, et al. Objective measurements of daily physical activity patterns and sedentary behaviour in older adults: Age, Gene/Environment Susceptibility-Reykjavik Study. Age Ageing. 2012;0:1-7.

15. Hu FB, Leitzmann MF, Stampfer M, Colditz GA, Willett WC, Rimm EB. Physical activity and television watching in relation to risk for type 2 diabetes mellitus in men. Arch Intern Med. 2001;161:1542-8.

16. Ford ES, Kohl HW, Mokdad AH, Ajani UA. Sedentary behavior, physical activity, and the metabolic syndrome among U.S. adults. Obes Res. 2005;13(3):608-14.

17. Edwardson CL, Gorely T, Davies M, Gray L, Khunti K, Wilmot EG, et al. Association of sedentary behaviour with metabolic syndrome: A meta-analysis. PLoS One. 2012;7(4):e34916.

18. Healy GN, Dunstan DW, Salmon J, Shaw JE, Zimmet PZ, Owen N. Television time and continuous metabolic risk in physically active adults. Med Sci Sports Exerc. 2008;40(4):639-45.

19. Dunstan DW, Salmon J, Owen N, Armstrong T, Zimmet PZ, Welborn TA, et al. Associations of TV viewing and physical activity with the metabolic syndrome in Australian adults. Diabetologia. 2005;48(1 1):2254-61.

20. Grøntved A. Television viewing and risk of type 2 diabetes, cardiovascular disease, and all-cause mortality. JAMA. 2011 ;305(23):2448-55.

21. Wilmot EG, Edwardson CL, Achana FA, Davies M, Gorely T, Gray L, et al. Sedentary time in adults and the association with diabetes, cardiovascular disease and death: Systematic review and meta-analysis. Diabetologia. 2012;55(1 1):2895-905

22. Ford ES, Caspersen CJ. Sedentary behaviour and cardiovascular disease: A review of prospective studies. Int J Epidemiol. 2012;41(5):1338-53.

23. Dunstan DW, Barr ELM, Healy GN, Salmon J, Shaw JE, Balkau B, et al. Television viewing time and mortality: The Australian Diabetes, Obesity and Lifestyle Study (AusDiab). Circulation. 2010;121(3):384-91. 
24. Hamilton MT, Healy GN, Dunstan DW, Zderic TW, Owen N. Too little exercise and too much sitting: Inactivity physiology and the need for new recommendations on sedentary behavior. Curr Cardiovasc Risk Rep. 2008;2(4):292-8.

25. Owen N, Bauman A, Brown W. Too much sitting: A novel and important predictor of chronic disease? Br J Sports Med. 2009;43(2):81-3.

26. Owen N, Healy GN, Matthews CE, Dunstan DW. Too much sitting: The population health science of sedentary behavior. Exerc Sport Sci Rev. 2010;38(3):105-13.

27. Owen N. Sedentary behavior: Understanding and influencing adults' prolonged sitting time. Prev Med. 2012;55(6):535-9.

28. Atkin AJ, Gorely T, Clemes SA, Yates T, Edwardson C, Brage S, et al. Methods of measurement in epidemiology: Sedentary behaviour. Int J Epidemiol. 2012;41(5):1460-71.

29. Healy GN, Clark BK, Winkler EAH, Gardiner PA, Brown WJ, Matthews CE. Measurement of adults' sedentary time in population-based studies. Am J Prev Med. 2011 1;41 (2):216-27.

30. Butte NF, Ekelund U, Westerterp KR. Assessing physical activity using wearable monitors: Measures of physical activity. Med Sci Sports Exerc. 2012;44(1 Suppl 1):S5-12.

31. Freedson PS, Melanson E, Sirard J. Calibration of the computer science and applications, inc. accelerometer. Med Sci Sport Exerc. 1998;30(5):777-81.

32. Ainsworth $B E$, Haskell $W L$, Whitt $M C$, Irwin $M L$, Swartz ANNM, Strath SJ, et al. Compendium of physical activities: An update of activity codes and MET intensities. Med Sci Sports Exerc. 2000;32(Suppl 9):S498-504.

33. Troiano RP, Berrigan D, Dodd KW, Mâsse LC, Tilert T, McDowell M. Physical activity in the United States measured by accelerometer. Med Sci Sports Exerc. 2008;40(1):181-8.

34. PAL Technologies Ltd. activPAL Operating Guide. 2010.

35. Kozey-Keadle S, Libertine A, Lyden K, Staudenmayer J, Freedson PS. Validation of wearable monitors for assessing sedentary behavior. Med Sci Sport Exerc. $2011 ; 43(8): 1561-7$.
36. Godfrey A, Culhane KM, Lyons GM.

Comparison of the performance of the activPAL $L^{T M}$ Professional physical activity logger to a discrete accelerometer-based activity monitor. Med Eng Phys. 2007;29(8):930-4.

37. Bankoski A, Harris TB, McClain JJ, Brychta RJ, Caserotti P, Chen KY, et al. Sedentary activity associated with metabolic syndrome independent of physical activity. Diabetes Care. $2011 ; 34(2): 497-503$

38. Healy $\mathrm{GN}$, Winkler $\mathrm{EAH}$, Brakenridge $\mathrm{CL}$, Reeves MM, Eakin EG. Accelerometer-derived sedentary and physical activity time in overweight/obese adults with type 2 diabetes: Cross-sectional associations with cardiometabolic biomarkers. PLoS One. 2015;10:e0119140.

39. Healy G, Dunstan D, Salmon J, Cerin E, Shaw J, Zimmet $\mathrm{P}$, et al. Breaks in sedentary time. Diabetes Care. 2008;31 (4):661-3.

40. Benatti F, Ried-Larsen M. The effects of breaking up prolonged sitting time. Med Sci Sport Exerc. 2015;47(10):2053-61

41. Dunstan DW, Salmon J, Healy GN, Shaw $J E$, Jolley D, Zimmet PZ, et al. Association of television viewing with fasting and $2-\mathrm{h}$ postchallenge plasma glucose levels in adults without diagnosed diabetes. Diabetes Care. 2007;30(3):516-22.

42. Dunstan DW, Salmon J, Owen N, Armstrong $T$, Zimmet PZ, Welborn, Timothy A Camerson AJ, et al. Physical activity and television viewing in relation torisk of undiagnosed abnormal glucose metabolism in adults. Diabetes Care. 2004;27(1 1):2603-9.

43. Schram MT, Sep SJS, van der Kallen CJ, Dagnelie PC, Koster A, Schaper N, et al. The Maastricht Study: An extensive phenotyping study on determinants of type 2 diabetes, its complications and its comorbidities. Eur J Epidemiol. 2014;29(6):439-51.

44. Harris TB, Launer $L$, Eiriksdottir $G$, Kjartansson O, Jonsson P, Sigurdsson G, et al. Age, Gene/ Environment Susceptibility-Reykjavik Study: Multidisciplinary applied phenomics. Am J Epidemiol. 2007;165(9): 1076-87. 
Chapt

照 


\section{AB STRACT}

As accelerometers are commonly used for $24 \mathrm{~h}$ measurements of daily activity, methods for separating waking from sleeping time are necessary for correct estimations of total daily activity levels accumulated during the waking period. Therefore, an algorithm to determine wake and bed times in $24 \mathrm{~h}$ accelerometry data was developed and the agreement of this algorithm with self-report was examined. One hundred seventy-seven participants (aged 40-75 years) of The Maastricht Study who completed a diary and who wore the activPAL3 ${ }^{\text {TM }} 24 \mathrm{~h} /$ day, on average 6 consecutive days were included. Intraclass correlation coefficient (ICC) was calculated and the Bland-Altman method was used to examine associations between the self-reported and algorithm-calculated waking hours. Mean self-reported waking hours was $15.8 \mathrm{~h} /$ day, which was significantly correlated with the algorithm-calculated waking hours ( $15.8 \mathrm{~h} /$ day, ICC $=0.79, \mathrm{P}<0.001$ ). The Bland-Altman plot indicated good agreement in waking hours as the mean difference was $0.02 \mathrm{~h}(95 \% \mathrm{LoA}=-1.1$ to $1.2 \mathrm{~h})$. The median of the absolute difference was $15.6 \mathrm{~min}(\mathrm{Q} 1-\mathrm{Q} 3=7.6-33.2 \mathrm{~min}$ ), and $71 \%$ of absolute differences was less than $30 \mathrm{~min}$. The newly developed automated algorithm to determine wake and bed times was highly associated with self-reported times, and can therefore be used to identify waking time in $24 \mathrm{~h}$ accelerometry data in large-scale epidemiological studies. 


\section{INTRODUCTION}

A growing number of studies have used accelerometers for objective assessments of sedentary behaviour and physical activity. ${ }^{1-3}$ Traditionally, participants are instructed to remove the device before going to bed and during water-based activities, and replace the device afterwards. However, when using this method potentially important behaviours, such as sleep, will not be collected. Furthermore, data on the waking period could be missed when participants forget to replace the device, which could result in an underestimation of total daily activity. ${ }^{4}$ Thus, to improve compliance and obtain more accurate assessment of total daily activity, studies should ideally use an accelerometer that can be worn 24 hours per day without removing the device at any time.

The activPALTM physical activity monitor (PAL Technologies, Glasgow, UK) is a small and lightweight accelerometer that can be made waterproof and can be attached to the skin. ${ }^{5}$ Consequently, this device can be worn continuously for a period of multiple days, providing complete assessments of all daily performed activity. Although the $24 \mathrm{~h}$ measurement overcomes the issues of incomplete assessments, it results in the challenge of how to identify the waking time in the $24 \mathrm{~h}$ period. As the activPAL has shown to be highly accurate in determining sedentary time and distinguish this from standing and stepping time, ${ }^{6,7}$ the posture allocation will classify both periods of sitting and lying, and thus also sleeping periods, as sedentary time. It is therefore important to identify waking time and separate this from the sleeping period (time in bed) in order to correctly estimate the amount of sedentary time and physical activity accumulated during the waking period.

Currently used methods to identify waking time include a fixed-time window (for example time between $7.00 \mathrm{~h}$ and $23.00 \mathrm{~h}),{ }^{8-10}$ a diary, or manual (visual) assessment of wake and bed times when analysing the data. ${ }^{11}$ However, using a fixed-time window could easily result in over- or underestimation of the waking period, and/or misclassification of sleeping time as waking (sedentary) time or vice versa, resulting in incorrect estimations of sedentary behaviour and physical activity. Using diaries increases burden for participants as well as researchers, and manual assessments of wake and bed times are not feasible in studies with a large sample size. In addition, a recently published study in children (such data is not available in an adult population) demonstrated that estimations of the amount of sedentary behaviour differ considerably depending on which of these methods was used. $^{12}$ 
Methods for identifying sleep have been described for wrist-worn ${ }^{13-15}$ and hip-14,16 or waist-worn devices. ${ }^{15,17-19}$ However, a valid method to determine wake and bed times for identifying waking time or time in bed has not yet been developed for accelerometers that are worn on the lower body, such as the activPAL. Furthermore, automatic methods to identify waking time on an individual level, i.e., for each day for each participant, are necessary for the analysis of large-scale datasets. Therefore, an automated algorithm was developed to determine wake and bed times in $24 \mathrm{~h}$ accelerometry data assessed with the activPAL3. In this study the agreement of this algorithm to determine wake and bed times with self-report was examined and this algorithm was compared with a fixed-time window, using a subsample of The Maastricht Study.

\section{METHODS}

\section{Study population}

For this study a subsample of The Maastricht Study was used. The Maastricht Study is an observational prospective population-based cohort study. The rationale and methodology have been described elsewhere. ${ }^{20}$ In short, the study focuses on the causes, pathophysiology, complications and comorbidities of type 2 diabetes mellitus and is characterized by an extensive phenotyping approach. Eligible for participation were all individuals aged between 40 and 75 years and living in the southern part of the Netherlands. The present study included participants who were asked to wear an accelerometer for eight consecutive days and complete a diary for wake and bed times between April and July 2013 ( $n=199$ ). After excluding participants who did not wear the accelerometer at least three days or who did not complete the diary for at least three days, a total of 177 participants were included.

The study has been approved by the Institutional Medical Ethical Committee (NL3 1329.068.10) and the Minister of Health, Welfare and Sports of the Netherlands, on the basis of the Health Council's opinion (Permit $131088-105234-P G)$. All participants gave written informed consent.

\section{Measurements}

The activPAL3 is a small $(53 \times 35 \times 7 \mathrm{~mm})$, lightweight $(15 \mathrm{~g})$ triaxial accelerometer that records movement in the vertical, anteroposterior and mediolateral axes, and 
also determines posture (sitting/lying, standing and stepping) based on acceleration information. The device was attached directly to the skin on the front of the right thigh with transparent $3 \mathrm{M}$ Tegaderm ${ }^{\mathrm{TM}}$ tape, after the device had been waterproofed using a nitrile sleeve. Participants were asked to wear the accelerometer for 8 consecutive days, without removing the device at any time. To avoid inaccurately identifying of non-wear time, participants were asked not to replace the device once removed. Data were uploaded using the activPAL software and processed using customised software written in MATLAB R2013b (MathWorks. Natick, MA, USA). Data from the first day were excluded from analysis because participants performed physical function tests at the research centre after the device was attached. In addition, data from the final wear day providing $<14 \mathrm{~h}$ of waking data were excluded from the analysis.

In addition, participants were asked to write down the exact time (hh:mm) they went to bed (bed time) and the time they got out of bed (wake time) in a diary on each day when wearing the accelerometer.

Algorithm-determined wake and bed times

To automatically determine wake and bed times on an individual level on multiple days (i.e., different wake and bed times, for each day, for each participant), an algorithm written for MATLAB was developed, using the activPAL3 events file, that indicates every event (a continuous period of time in which one type of activity is executed, e.g., sitting/ lying, standing or one step) of any time duration. Rules and thresholds used in the final version of the algorithm were accomplished by visually comparing output of our algorithm with manually assessed output using the activPAL software on which the algorithm was further developed. This procedure was repeated multiple times using different subsets ( $n=500)$ of The Maastricht Study dataset, other than the sample used in the current study. Further, we tested our algorithm by generating sample data in order to evaluate the algorithm's performance, and this demonstrated that our algorithm treated all data as expected (e.g., correctly determined wake and bed times). The algorithm is available for other researchers and can be obtained from the authors. A brief description of the algorithm is given below.

Bed time was based on sedentary bouts of sitting/lying time (sed-bout). At first, the software identified 5 consecutive sed-bouts of any duration (minimum $1 \mathrm{sec}$ ) using a moving window between 19:00 hand 12:00 h (noon). Next, the duration of each individual bout (sed-bout ${ }_{1}-$ sed-bout $_{5}$ ) as well as the sum of duration of sed-bout ${ }_{1-2}$, sed-bout $t_{1-3}$, sed-bout $_{1-4}$ or sed-bout ${ }_{1.5}$ was determined. These values were compared with the pre-set cut-off points 
(see Table 2.1). If sed-bout ${ }_{1}$ exceeded a cut-off point, bed time was set at the start time of sed-bout ${ }_{1}$. If sed-bout ${ }_{1-2}$ exceeded a cut-off point, bed time was set at the start time of sed-bout $_{1}$, if the duration of sed-bout ${ }_{1}>1 \mathrm{~h}$. However, when the duration of sed-bout ${ }_{1}<1 \mathrm{~h}$, bed time was set at start time of sed-bout ${ }_{2}$. If sed-bout ${ }_{1-3}$ or sed-bout ${ }_{1-4}$ or sed-bout $_{1^{-} 5}$ exceeded a cut-off point and all the sed-bouts had a duration $>1 \mathrm{~h}$, bed time was set at start time of sed-bout ${ }_{1}$. If one of the sed-bouts had a duration $<1 \mathrm{~h}$, the software repeated the procedure by identifying sed-bout ${ }_{2-6}$ and further, until bed time was determined. When no following sed-bouts were available, bed time was set at the start time of the first sed-bout of the final five sed-bouts that were identified. When no five sed-bouts were available at all, the sum of the duration of the available sed-bouts was calculated, and when this sum was $\geq 4 \mathrm{~h}$, bed time was set at the start time of the first sed-bout. When the sum of the sedbouts was $<4 \mathrm{~h}$, no bed time was determined. The algorithm was adapted to the following exceptions: 1) short periods of standing or stepping during the night (for example toileting) were allowed if the total duration $<6 \mathrm{~min} ; 2$ ) standing or stepping with a duration $\geq 15$ min which occur before 0 1:00 h and after a sed-bout $\geq 2.5 \mathrm{~h}$ (e.g., watching TV before going to bed) were classified as waking time.

Wake time was based on bouts of standing or stepping time (act-bout). At first, 5 sed-bouts starting from 00:00 $\mathrm{h}$ were identified, of which the sum of the total duration must be $\geq 4 \mathrm{~h}$. Then, the start time of sed-bout ${ }_{1}$ was determined, and from that point, act-bouts were identified. Next, the act-bouts were tested against 10 pre-set cut-off points for total duration of act-bouts, as well as the duration of act-bouts as percentage of the total time in which these act-bouts occurred (sum of duration of act-bouts and duration of sed-bout, which occurred in-between the act-bouts) (see Table 2.1). The start time of the first act-bout that exceeded one of the 10 cut-off points, was set as wake time. When no act-bout exceeded a cut-off point, wake time was set at the end time of the 5 sed-bouts. The algorithm was adapted to the following exception: act-bouts between 03:00 $\mathrm{h}$ and 06:00 $\mathrm{h}$ which were followed by a sed-bout $\geq 2 \mathrm{~h}$, were classified as sleeping time.

Waking time was calculated as the time between wake time and bed time.

\section{Self-reported wake and bed times}

A diary was used to obtain self-reported wake and bed times. Waking time was calculated as the time between wake time and bed time. 
Table 2.1: Cut-off points for determining wake and bed times in the proposed algorithm

\begin{tabular}{|c|c|c|}
\hline Start time cluster & & Sum of duration sed-bouts (h) \\
\hline \multicolumn{3}{|l|}{ Bed time } \\
\hline 23:00 $\mathrm{h}$ and later & & $4 \mathrm{~h}$ \\
\hline $22: 00-23: 00 \mathrm{~h}$ & & $5 \mathrm{~h}$ \\
\hline $21: 00-22: 00 \mathrm{~h}$ & & $6 \mathrm{~h}$ \\
\hline 19:00-21:00 h & & $7 \mathrm{~h}$ \\
\hline Act-bout (n) & $\begin{array}{l}\text { Sum of duration } \\
\text { of act-bout (min) }\end{array}$ & $\begin{array}{l}\text { Percentage of } \\
\text { total time }\end{array}$ \\
\hline \multicolumn{3}{|l|}{ Wake time } \\
\hline 1 & 10 & 40 \\
\hline 2 & 5.0 & 20 \\
\hline 3 & 5.2 & 10 \\
\hline 4 & 5.4 & 8 \\
\hline 5 & 5.6 & 8 \\
\hline 6 & 5.8 & 8 \\
\hline 7 & 6.0 & 8 \\
\hline 8 & 6.2 & 8 \\
\hline 9 & 6.4 & 8 \\
\hline 10 & 6.6 & 8 \\
\hline
\end{tabular}

Sed-bout, bout of sitting/lying; act-bout, bout of standing or stepping. $n=177$.

Fixed wake and bed times

The activPAL3 data was also processed with customized software written in MATLAB that set for each participant their daily wake time and bed time at 07:00 $\mathrm{h}$ and 23:00 $\mathrm{h}$. According to this method, waking time was $16 \mathrm{~h}$ for each participant on each day.

\section{Sedentary time}

Sedentary time was based on the posture data (sitting/lying), and determined as the total time spent in a sedentary position between wake time and bed time. Sedentary time and percentage sedentary time of total waking time were calculated for both algorithmdetermined and fixed-time window methods.

\section{Other variables}

Of the 177 participants the following variables were obtained as described elsewhere: ${ }^{20}$ sex, age (at first day of wearing the activPAL3), and level of education. Level of education 
was categorized as low, medium, and high. Weight and height were measured without shoes and wearing light clothing using a scale and stadiometer to the nearest $0.5 \mathrm{~kg}$ or $0.1 \mathrm{~cm}$ (Seca, Hamburg, Germany). Body mass index (BMI) was calculated as kilogram per meter squared $\left(\mathrm{kg} / \mathrm{m}^{2}\right)$.

\section{Statistical analysis}

Descriptive characteristics of the study sample, and their provided self-reported and accelerometry data were summarized as mean and standard deviation (SD) or percentages. The absolute differences between wake times and bed times derived from the algorithm-determined, self-report, and fixed-time window methods were calculated and described by range, mean and SD, and median and interquartile range. Absolute differences can provide more useful insights in differences between two methods than mean differences as the latter may result in a value around 0 when over- and underestimations balance each other out.

Waking time was described (range, mean, SD) for all three methods (algorithm-determined, self-report, fixed-time window), and the association between algorithm-determined and self-reported waking time was examined by calculating intraclass correlation coefficients (ICC). Absolute differences and absolute percentage differences in waking time between the algorithm-determined method and the self-report or fixed-time window method were calculated and described by range, mean and SD, and median and interquartile range. The Bland-Altman method was used to determine level of agreement between algorithmdetermined and self-reported waking time.

Total amount of sedentary time and percentage sedentary time as determined with the algorithm-determined and the fixed-time window methods were described by range, mean and SD, and median and interquartile range. The absolute differences and absolute percentage differences in sedentary time and percentage sedentary time between these methods were calculated and described by range, mean and SD, and median and interquartile range. The Bland-Altman method was used to determine level of agreement between sedentary time as determined by algorithm and fixed-time window methods. All analyses were conducted with IBM SPSS Statistics 22.0 (IBM Corp. Armonk, NY, USA). 


\section{$\underline{\text { RESULTS }}$}

A total of 177 participants completed a diary and wore the accelerometer on average 6 days. Other general characteristics of the study sample are shown in Table 2.2. This study sample was similar with regard to sex, age, level of education and BMI, compared to The Maastricht Study sample that consists of 3,451 participants who completed the baseline survey between November 2010 and September 2013.

Table 2.2: Descriptive characteristics of the study sample

\begin{tabular}{|c|c|c|}
\hline$n$ & 177 & \\
\hline Female & 53.1 & $\%$ \\
\hline Age (years) & 59.4 & $(7.5)$ \\
\hline \multicolumn{3}{|l|}{ Level of education* } \\
\hline - low & 34.1 & $\%$ \\
\hline - medium & 28.3 & $\%$ \\
\hline - high & 37.6 & $\%$ \\
\hline BMI $\left(\mathrm{kg} / \mathrm{m}^{2}\right)$ & 27.1 & $(4.6)$ \\
\hline Number of days with self-reported data & 6.9 & $(0.6)$ \\
\hline Number of days with accelerometry data & 6.7 & $(0.7)$ \\
\hline
\end{tabular}

Values are mean $(S D)$, unless stated otherwise. $B M l$, body mass index. ${ }^{*} n=173$.

\section{Algorithm-determined versus self-reported methods}

Table 2.3 shows the absolute differences between the wake and bed times derived from the algorithm-determined, self-report, and fixed-time window methods. The median of the absolute differences between the self-reported and algorithm-determined wake time was $12 \mathrm{~min}$, which indicates that in half of the participants the algorithm-determined wake time differed less than 12 min from their self-reported wake time. For bed times, the algorithmdetermined method differed less than $25 \mathrm{~min}$ from the self-reported bed times in half of the participants. As presented in Table 2.4, mean (SD) waking time per day was $15.8 \mathrm{~h}(0.9)$ when using the algorithm-determined method, which was comparable with and statistically significantly correlated to the self-reported waking time (15.8 h (0.9); ICC $=0.79 ; P<0.001$ ). The median of the absolute differences in waking hours between the algorithm-determined and self-report methods was $16 \mathrm{~min}(\mathrm{Q} 1-\mathrm{Q} 3=7.6-33.2 \mathrm{~min})$, and $71 \%$ of these 
absolute differences in all participants was less than $30 \mathrm{~min}$. Further, the median of the absolute percentage difference was 1.7\% (Q1-Q3 = $0.8-3.5 \%)$. Finally, the BlandAltman plot indicates good agreement between the algorithm-determined and self-reported waking hours, with a mean difference of $0.02 \mathrm{~h}$ (1.2 min) and limits of agreement of -1.1 to $1.2 \mathrm{~h}$ (Figure 2.1).

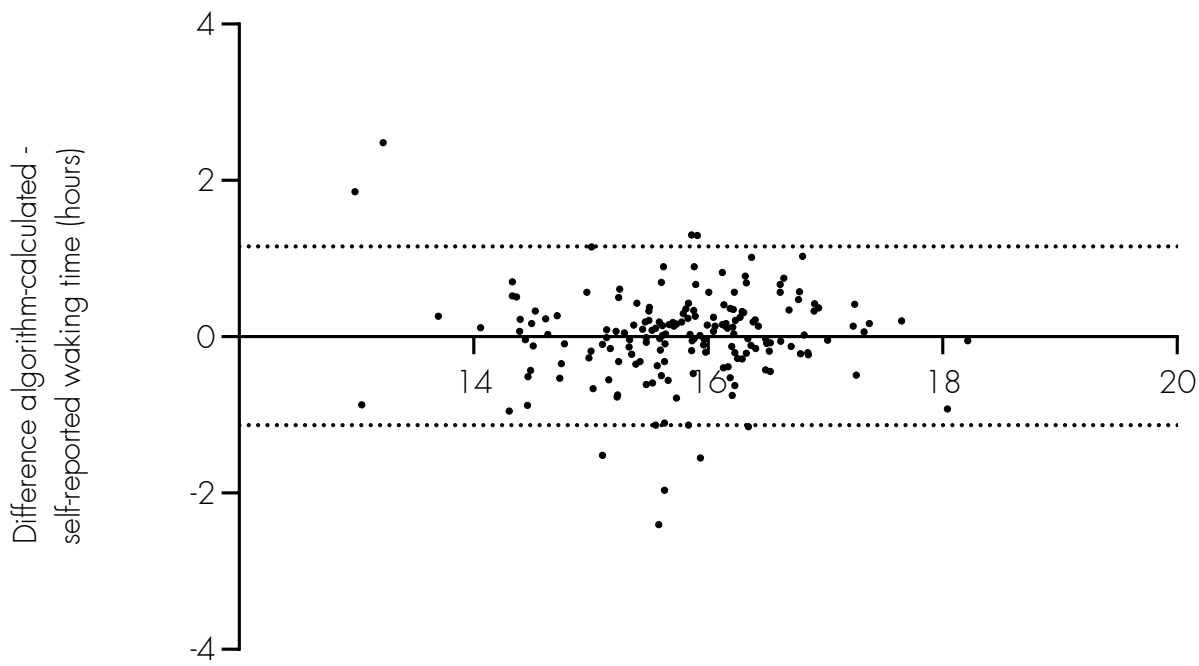

Mean algorithm-calculated and self-reported waking time (hours)

Figure 2.1: Bland-Altman plot of the mean of and differences between waking hours based on self-reported and algorithm-calculated waking time. Dotted lines indicate the limits of agreement. 

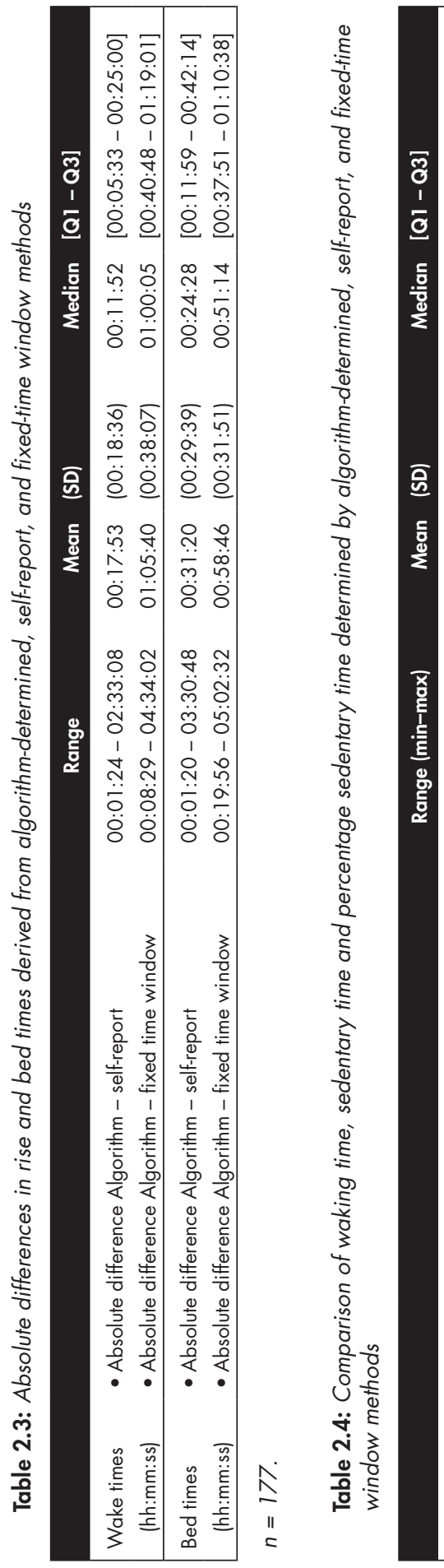

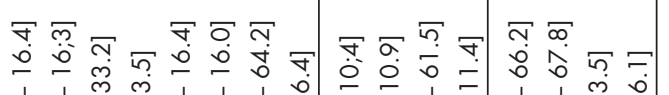

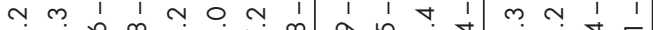

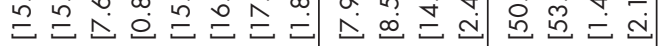
\.

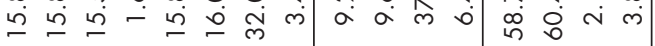

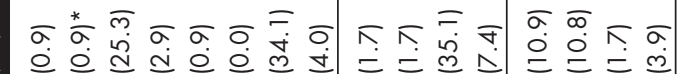

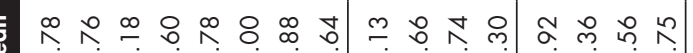
ก

ก

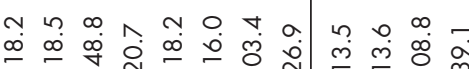
m.

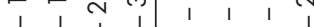

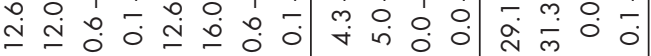

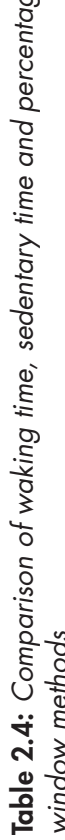

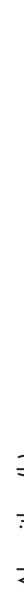

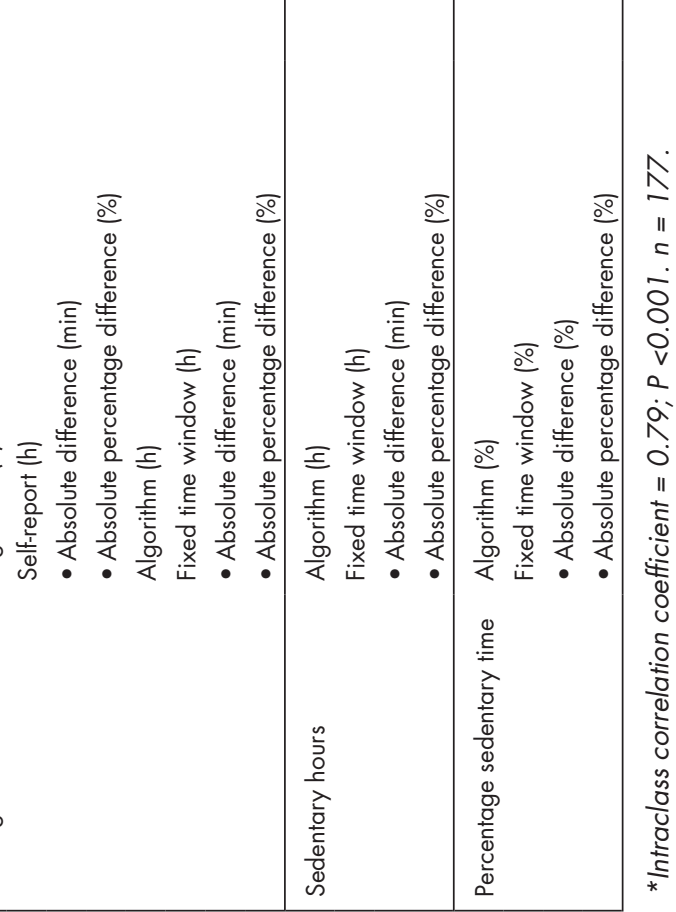




\section{Algorithm-determined versus fixed-time window methods}

The fixed-time window method determined wake time at 7:00 h and bed time at 23:00 $\mathrm{h}$ for each participant on each day, thus mean waking hours was $16.0 \mathrm{~h}$. Table 2.4 shows that the median of the absolute differences in waking hours between the algorithmdetermined and fixed-time window methods was $32 \min (Q 1-Q 3=17.2-64.2 \mathrm{~min}$ ) and the median of the absolute percentage difference was 3.4\% (Q1-Q3 $=1.8-6.4 \%)$. However, Table 2.3 shows substantial differences in the absolute differences between wake and bed times determined with both methods: the median of the absolute differences was $1 \mathrm{~h}$ for wake times, and $51 \mathrm{~min}$ for bed times. Furthermore, $26 \%$ of the algorithm-determined wake times was before $7: 00 \mathrm{~h}$, and $69 \%$ of the algorithm-determined bed times was after 23:00 $\mathrm{h}$ (data not tabulated). These results indicate that although the estimated total amount of waking time of $16 \mathrm{~h}$ by the fixed-time window method was comparable with the algorithm-determined waking time (i.e., $15.8 \mathrm{~h}$ ), the determination of the waking period during the day differed substantially between the methods as illustrated in Figure 2.2. The figure shows that when, for example, wake time was before 7:00 h and bed time was before 23:00 h, the fixed-time window method misclassified waking time as sleeping time, and sleeping time as waking (sedentary) time. Similar misclassifications could occur with all wake and bed times other than 7:00 h and 23:00 h. Consequently, the amount of sedentary time and the percentage sedentary time were overestimated by the fixed-time window method in comparison with the algorithm-determined sedentary time, because standing/stepping time before 7:00 $\mathrm{h}$ and after 23:00 $\mathrm{h}$ was classified as sleeping time and therefore excluded from analysis (Table 2.4 and Figure 2.3).

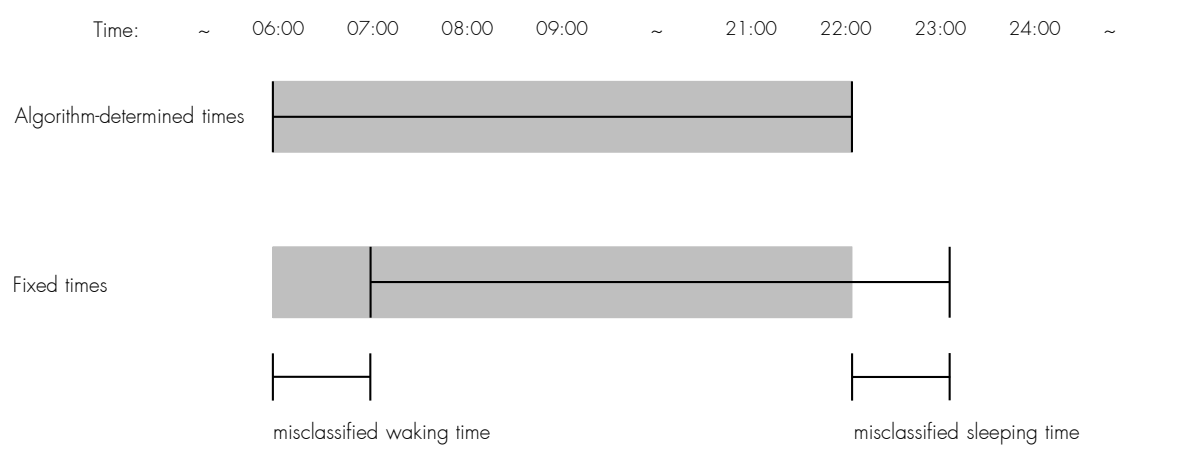

Figure 2.2: Illustration of misclassifying waking and sleeping time by the fixed-time window method compared to the algorithm-determined method. Grey bars indicate waking time. 


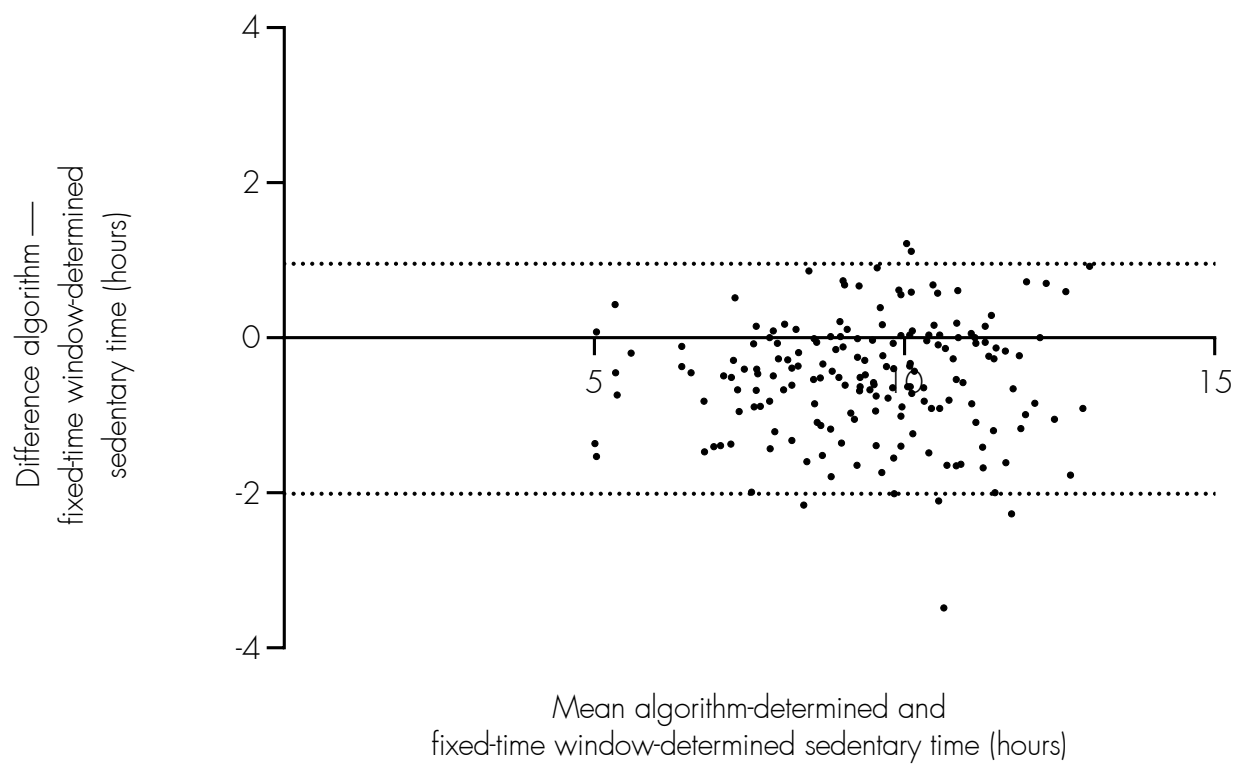

Figure 2.3: Bland-Altman plot of the mean of and differences between sedentary time as determined by the algorithm an fixed-time window methods. Dotted lines indicate the limits of agreement.

\section{DISCUSSION}

This study describes the development of an algorithm to automatically determine wake and bed times in $24 \mathrm{~h}$ accelerometry data assessed using the activPAL3 accelerometer.

The algorithm achieved high levels of agreement in determining wake times (median of the absolute difference was $12 \mathrm{~min}$ ) as well as bed times (median of the absolute difference was $25 \mathrm{~min}$ ) when compared to self-reported diary times. In addition, the algorithm-calculated waking time was highly correlated with the self-reported time, and the mean difference was $0.02 \mathrm{~h}(1.2 \mathrm{~min})$. Furthermore, $71 \%$ of the absolute differences in waking time was less than $30 \mathrm{~min}$, and the median of the absolute percentage difference was $1.7 \%$. These results indicate that the algorithm can be used as an accurate method to automatically identify the waking period and separate this from the sleeping period in $24 \mathrm{~h}$ activPAL accelerometry data, in middle-aged and older adults when compared to a self-reported method. Consequently, when using the algorithm the amounts of sedentary 
time and physical activity accumulated during waking time can be estimated correctly, which is of importance for examining associations between sedentary behaviour or physical activity and health outcomes. As there currently is no other method available to automatically separate the waking from the sleeping period for data assessed with the activPAL, the algorithm is based on rules and thresholds developed in subsets of The Maastricht Study dataset.

Further, the algorithm was compared to a fixed-time window for wake $(07: 00 \mathrm{~h})$ and bed $(23: 00 \mathrm{~h})$ times. The results showed considerable differences in determined wake and bed times between the methods, as the median of the absolute difference was $1 \mathrm{~h}$ for wake, and $51 \mathrm{~min}$ for bed times. However, calculated waking time was comparable ( $16 \mathrm{~h}$ vs $15.8 \mathrm{~h}$ ) between the methods. This indicates that the determination of the waking period during the day differs highly when using a fixed-time window instead of the algorithm. As a result, the fixed-time window method could misclassify actual waking time before 07:00 h and after 23:00 h as sleeping time, and actual sleeping time after 07:00 $\mathrm{h}$ and before $23: 00 \mathrm{~h}$ as (sedentary) waking time. When only waking time is analysed, using a fixed-time window could thus result in an overestimation of sedentary time in comparison with the algorithm.

To date, a method to identify waking time in $24 \mathrm{~h}$ data provided by an accelerometer that is worn on the lower body, has not been developed. Methods to determine sleep or time in bed using hip-worn or waist-worn accelerometers have been described before, ${ }^{13-19}$ and some methods have been shown to be fairly accurate. ${ }^{15,17}$ Only Barreira and colleagues ${ }^{17}$ reported mean differences, and these were larger than the observed mean differences in this study (i.e., $7 \pm 29 \mathrm{~min}$ ). Further, these methods were validated in children and were developed for the ActiGraph accelerometer, based on counts per minute, and could therefore not be used in adults and for data on posture assessed with the activPAL. Another possibility to identify waking time is using a fixed-time window, which have been used in studies with a $24 \mathrm{~h}$ wear time protocol. ${ }^{8-10}$ However, this study shows that a fixed-time window could result in misclassifications of waking and/or sleeping time, and in overestimations of sedentary time. Other methods include the use of diaries or manual assessment of the waking period, ${ }^{11}$ but these methods are not appropriate for large-scale studies because of the burden for participants as well as researchers to collect, enter and clean the data. The algorithm overcomes these disadvantages as it allows automatic identification of waking time on each day and for each participant, which reduces time for analysis considerably. Consequently, accurate assessment of $24 \mathrm{~h}$ measurements of 
sedentary behaviour and/or physical activity using the activPAL becomes feasible in large-scale studies when using the algorithm.

A strength of this study was the use of data on posture provided by the activPAL3 to develop the algorithm. As the device uses posture rather than movement, the accuracy for detecting a lying position is increased, which is essential for a more precise identification of time spent in bed. Another strength was that almost all participants (93\%) provided 6 days with self-reported and accelerometry data, which enables us to examine our algorithm in several participants on multiple days.

A few limitations of this study should also be mentioned. The algorithm is not able to determine wake and/or bed times when the accelerometer was worn for only a few hours on a day. For example, no wake time could be determined if the accelerometer was removed shortly after the actual wake time, because of a lack of data to process. Further, wake time and bed times could be determined incorrectly as the rules used in the algorithm were not appropriate for each individual. For example, when only short amounts of time with activity (standing or stepping) were accumulated after the actual wake time, or when several periods of activity were accumulated after the actual bed time, the algorithm determined wake or bed times at a later time than the actual time. This resulted in an absolute difference between self-reported and algorithm-determined times of at most $3.5 \mathrm{~h}$. The algorithm may therefore not be applicable for extremely sedentary individuals or individuals with considerable amounts of activity (standing, stepping) during the night. Another limitation is that the self-report method to obtain wake and bed times which we have used as a reference method, could have been subject to recall bias or were incomplete. However, self-report is a commonly used method in epidemiological studies, and is considered as a standard method to obtain data about the sleeping period. ${ }^{21}$ Nevertheless, to further validate the algorithm it should be tested against direct observation or polysomnography, although the latter provides estimates of actual sleep rather than the time between participants went to bed and went out of bed. Finally, applicability of the algorithm in other populations (e.g., children and youth), and to other accelerometers should be examined in future studies. 


\section{CONCLUSIONS}

This study showed that a newly developed algorithm to automatically determine wake and bed times on an individual level in $24 \mathrm{~h}$ accelerometer data was highly associated with self-reported wake and bed times. Therefore, the algorithm could be used as an accurate method to identify waking time when compared to self-reported methods, which overcomes issues of incomplete assessments (differentiate wear time from non-wear time) and could improve compliance for obtaining appropriate assessments. As a result, using an accelerometer with a $24 \mathrm{~h}$ wear time protocol becomes feasible, even in large-scale epidemiological studies. 


\section{REFERENCES}

1. Lee I-M, Shiroma EJ. Using accelerometers to measure physical activity in large-scale epidemiological studies: Issues and challenges. Br J Sports Med. 2014;48:197-201.

2. Bassett DR. Device-based monitoring in physical activity and public health research. Physiol Meas. 2012;33(11):1769-83.

3. Chen KY, Bassett DR. The technology of accelerometry-based activity monitors: Current and future. Med Sci Sport Exerc. 2005;37(Supplement):S490-500.

4. Tudor-Locke C, Barreira TV, Schuna JM, Mire EF, Chaput J-P, Fogelholm $M$, et al. Improving wear time compliance with a 24-hour waist-worn accelerometer protocol in the International Study of Childhood Obesity, Lifestyle and the Environment (ISCOLE). Int J Behav Nutr Phys Act. 2015;12(1 1):1 1 .

5. PAL Technologies Ltd. activPAL Operating Guide. 2010.

6. Kozey-Keadle S, Libertine A, Lyden K, Staudenmayer J, Freedson PS. Validation of wearable monitors for assessing sedentary behavior. Med Sci Sport Exerc. 2011 1;4(8):1561-7.

7. Godfrey A, Culhane KM, Lyons GM. Comparison of the performance of the activPAL ${ }^{T M}$ Professional physical activity logger to a discrete accelerometer-based activity monitor. Med Eng Phys. 2007;29(8):930-4.

8. De Decker E, De Craemer M, Santos-Lozano A, Van Cauwenberghe E, De Bourdeaudhuij I, Cardon G. Validity of the ActivPAL ${ }^{\mathrm{TM}}$ and the ActiGraph monitors in preschoolers. Med Sci Sports Exerc. 2013;45(10):2002-11.

9. Smith L, Thomas EL, Bell JD, Hamer M. The association between objectively measured sitting and standing with body composition: A pilot study using MRI. BMJ Open. 2014;4:e005476.

10. Smith L, Hamer M, Ucci M, Marmot A, Gardner B, Sawyer A, et al. Weekday and weekend patterns of objectively measured sitting, standing, and stepping in a sample of office-based workers: The active buildings study. BMC Public Health. 2015;15:1-9.

11. Dowd KP, Harrington DM, Bourke AK, Nelson J, Donnelly AE. The measurement of sedentary patterns and behaviors using the activPAL ${ }^{T M}$ Professional physical activity monitor. Physiol Meas. 2012;33(11):1887-99.
12. Meredith-Jones K, Williams S, Galland B, Kennedy G, Taylor R. 24 h Accelerometry: Impact of sleep-screening methods on estimates of sedentary behaviour and physical activity while awake. J Sports Sci. 2016;34(7):679-85.

13. Marino $M$, Li $Y$, Rueschman $M N$, Winkelman JW, Ellenbogen JM, Solet JM, et al. Measuring sleep: Accuracy, sensitivity, and specificity of wrist actigraphy compared to polysomnography. Sleep. 2013;36:1747-55.

14. Ray M, Youngstedt S, Zhang H, Wagner Robb S, Harmon B, Jean-Louisi $G$, et al. Examination of wrist and hip actigraphy using a novel sleep estimation procedure. Sleep Sci. 2014;7(2):74-81.

15. Tracy DJ, Xu Z, Choi L, Acra S, Chen KY, Buchowski MS. Separating bedtime rest from activity using waist or wrist-worn accelerometers in youth. PLoS One. 2014;9(4):e92512.

16. Kinder J, Lee K, Thompson H, Hicks K, Topp K, Madsen K. Validation of a hip-worn accelerometer in measuring sleep time in children. J Pediatr Nurs. 2012;27(2):127-33.

17. Barreira TV, Schuna JM, Mire EF, Katzmarzyk PT, Chaput J-P, Leduc G, et al. Identifying children's nocturnal sleep using 24-h waist accelerometry. Med Sci Sport Exerc. 2015;47(5):937-43.

18. Hiorth MF, Chaput JP, Damsgaard CT, Dalskov SM, Michaelsen KF, Tetens I, et al. Measure of sleep and physical activity by a single accelerometer: Can a waist-worn Actigraph adequately measure sleep in children? Sleep Biol Rhythms. 2012;10:328-35.

19. Tudor-locke C, Barreira TV, Schuna JM, Mire E, Katzmarzyk PT. Fully automated waist - worn accelerometer algorithm for detecting children's sleep period time separate from 24 - hour physical activity or sedentary behaviors. Appl Physiol Nutr Metab. 2014;39:53-7.

20. Schram MT, Sep SJS, van der Kallen CJ, Dagnelie PC, Koster A, Schaper N, et al. The Maastricht Study: An extensive phenotyping study on determinants of type 2 diabetes, its complications and its comorbidities. Eur J Epidemiol. 2014;29(6):439-51.

21. Devine EB, Hakim Z, Green J. A systematic review of patient-reported outcome instruments measuring sleep dysfunction in adults. Pharmacoeconomics. 2005;23(9):889-912. 
Chapt

照 

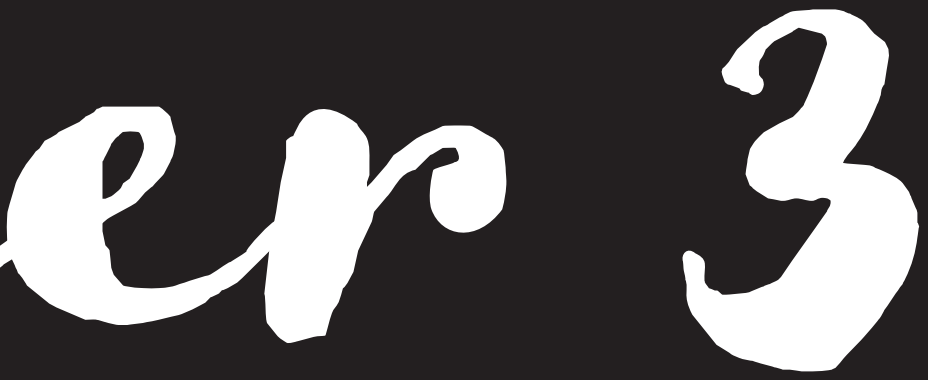

Measuring back-to-front and side-to-side movement during sedentary activities using a hip-worn tri-axial accelerometer

JULIANNE D. VAN DER BERG | COEN D.A. STEHOUWER | HANS BOSMA | ANNEMARIE KOSTER 


\section{AB STR ACT}

Background: In sedentary behaviour studies, a cut-off point of $<100$ counts per minute (cpm) in the vertical (up-and-down) axis has often been used to determine sedentary time. Although sedentary behaviour is characterized by no or low movement in the vertical axis (V-axis), back-to-front and side-to-side movement is likely to occur, for example when doing desk work. However, it is not known whether a hip-worn tri-axial accelerometer can record movement in the anteroposterior (back-to-front) and mediolateral (side-to-side) axes while being in a sedentary position. Therefore, we examined in a laboratory setting the amount of activity counts recorded in the anteroposterior (AP) and mediolateral (ML) axes using a hip-worn tri-axial accelerometer during common sedentary activities.

Methods: We included 10 men and 8 women (age (mean \pm SD): $27.3 \pm 6.5$ years). They wore a tri-axial ActiGraph accelerometer on the right hip and were asked to perform a protocol consisting of two controlled and seven common sedentary activities (total duration: $56 \mathrm{~min}$ ). For each activity the percentages sedentary $1<100 \mathrm{cpm}$ in all axes), active-sedentary $(\geq 100 \mathrm{cpm}$ in the AP and/or ML axes and $<100 \mathrm{cpm}$ in the V-axis), and active ( $\geq 100$ $\mathrm{cpm}$ in the V-axis) time were calculated.

Results: During the sedentary activities, predominantly sedentary time was recorded. However, during the activities 'watching TV', 'playing cards', and 'preparing food' up to $14 \%$ of time was classified as active-sedentary time.

Conclusions: During common sedentary activities a hip-worn tri-axial accelerometer recorded significant amounts of activity counts in the AP and/or ML axes. Therefore, we encourage researchers to include data of the $A P$ and $M L$ axes in order to distinguish those with more movement during sedentary time from those with less movement, as this may be relevant for health. To further verify the presence of active-sedentary time, studies are needed outside the laboratory setting and in other study populations. 


\section{INTRODUCTION}

Since several years the use of accelerometers for the objective measurement of physical activity and sedentary behaviour has become commonplace. The ActiGraph accelerometer (ActiGraph, Fort Walton Beach, FL, USA) is a commonly used device, most often worn on the hip where it records movement (e.g. activity counts) in three directions: vertical (up-and-down direction), anteroposterior (back-to-front), and mediolateral (side-to-side). To classify the movement into activities with different intensity levels (e.g. light physical activity (LPA) and moderate-to-vigorous physical activity (MVPA)), most studies have used cut-off points for the activity counts.

For sedentary behaviour, which is defined as any waking behaviour that is characterized by an low energy expenditure $\left(\leq 1.5\right.$ metabolic equivalents (METs)), ${ }^{1}$ a cutoff point of $<100$ counts per minute $(\mathrm{cpm})^{2}$ has most often been used. This cut-off point includes only data of the vertical axis ( $V$ axis) and movement in the other directions (backto-front and side-to-side) is not taken into account. However, movement with the upperbody in these directions is likely to occur during sedentary activities, for example when doing desk work or playing cards. So theoretically, a more comprehensive representation of sedentary behaviour can be obtained when taking into account activity accumulated in the anteroposterior (AP) and mediolateral (ML) axes, since it may be possible to distinguish between those with more movement during sedentary time and those with less movement.

Since adults spend large amounts of time sedentary ${ }^{3-5}$ which has been associated with adverse effects on cardio-metabolic biomarkers, ${ }^{6-8}$ cardiovascular disease, ${ }^{8,9}$ and mortality, 8,9 it could be important to use a more comprehensive representation of sedentary behaviour by taking into account back-to-front and side-to-side movement. This can contribute to further establish the associations between sedentary behaviour and health.

However, to date it is unknown whether a hip-worn accelerometer can record movement in the AP and ML axes while being in a sedentary position. Therefore, we examined in a laboratory setting the amount of activity counts recorded in the AP and ML axes by tri-axial hip-worn accelerometer, during common sedentary activities. 


\section{METHODS}

\section{Study population}

Participants of this study, employees of Maastricht University and Maastricht University Medical Centre, were recruited via advertisement and included when they were aged between 18 and 65 years. Participants with serious mobility limitations, defined as not able to walk, were excluded from participation. A total of 18 participants were included. This study was approved by the Local Ethics Committee of Maastricht University Medical Centre. All participants gave written informed consent.

\section{Study protocol}

Participants were seated on a chair without armrests and wearing an ActiGraph accelerometer (ActiGraph GT3X, Fort Walton Beach, FL, USA) on the right hip. Participants were asked to perform a protocol consisting of two controlled sedentary activities (controlled sitting and controlled fidgeting) and seven common sedentary activities (natural sitting, watching TV, reading, writing, typing, playing cards, and preparing food; see Table 3.1). For the activity 'controlled sitting' participants were instructed to sit completely still, thus without any movement of the body. The instructions for the activity 'controlled fidgeting' were: one minute of upper body movement from side to side, immediately followed by one minute of movement from back to front, repeated by one minute of side-to-side and one minute of back-to-front movement, all without moving the legs. Both activities were used to determine whether the accelerometer records activity in the three axes as expected. For the sedentary activity 'natural sitting' participants were asked to sit as they would normally do when no instructions were given. During the sedentary activities 'watching TV', 'reading', 'writing', 'typing', 'playing cards', and 'preparing food' participants were asked to perform the requested activity, while no restrictions in the movements were imposed. In-between the activities were participants asked to stand up three times. The total duration of the protocol was $56 \mathrm{~min}$. The device was synchronized with the protocol using the internal clock. The protocol was explained and directly observed by one trained research assistant. Before the protocol started weight, height, waist and hip circumference were measured. 
Table 3.1: Protocol of controlled and common sedentary activities

\begin{tabular}{|lll|}
\hline Duration (s) & Activity & Description \\
\hline 300 & Natural sitting & Participant sits and allowed to move freely \\
300 & Controlled sitting & Participant sits motionless, thus without moving arms or legs \\
240 & Controlled fidgeting & $\begin{array}{l}\text { Participant sits while moving the upper body from side-to-side (120 s) } \\
\text { and then from back-to-front (120 s) }\end{array}$ \\
420 & Reading & Participant sits while reading a newspaper \\
420 & Writing & Participant sits while writing a letter \\
420 & Watching TV & Participant sits while watching a TV show on a computer screen \\
420 & Playing cards & Participant sits while playing cards \\
420 & Typing & Participant sits while using mouse and keyboard to type and edit a text \\
420 & Preparing food & Participant sits while preparing fruit \\
\hline
\end{tabular}

\section{Measurements}

The tri-axial ActiGraph recorded activity on the vertical, anteroposterior (AP), and mediolateral $(\mathrm{ML})$ axes. Data of all axes were uploaded using the Actilife 6.6.1 software and activity counts were averaged per minute. Each minute of each activity was categorized as sedentary, active-sedentary or active. Sedentary minutes were defined as minutes with $<100$ counts in the V, AP and ML axes. Active-sedentary minutes were defined as minutes with activity of $\geq 100$ counts in the AP and/or ML axes and $<100$ counts in the $V$ axis. Active minutes were defined as minutes with $\geq 100$ counts in the $V$ axis.

Other variables included sex, age, body mass index (BMI), waist circumference and hip circumference. BMI was calculated using height and weight data $\left(\mathrm{kg} / \mathrm{m}^{2}\right)$ which were measured without shoes and wearing light clothing using a scale and stadiometer to the nearest $0.5 \mathrm{~kg}$ or $0.1 \mathrm{~cm}$ (Seca, Hamburg, Germany). Circumferences of the waist and hip were measured with a flexible plastic tape measure (Seca, Hamburg, Germany), between the lower rib margin and the iliac crest at the end of expiration, and at the level of the widest circumference over the greater trochanter, both to the nearest $0.5 \mathrm{~cm}$.

\section{Statistical analysis}

For each sedentary activity the percentage sedentary, active-sedentary, and active minutes was calculated. Next, the number and percentage of participants with one or more active-sedentary minutes and one or more active minutes were calculated for each activity to provide insight in the existence of active-sedentary and active minutes in the participants. To examine whether the activities differed from 'natural sitting', the number 
of participants with active-sedentary and active minutes during 'natural sitting' and the other sedentary activities were compared using McNemar tests. Also the average cpm per activity for each axis were described with the range, mean and standard deviation (SD), and median and interquartile range. Other data (sex, age, BMI, waist and hip circumference) were summarized and described as mean (SD) or numbers and percentages. All analyses were conducted with IBM SPSS Statistics 22.0 (IBM Corp. Armonk, NY, USA).

\section{RESULTS}

A total of 18 participants (10 men (55.6\%)) with an average age of $27.3 \pm 6.5$ years were included. Men had on average a BMI of $22.6 \pm 3.2 \mathrm{~kg} / \mathrm{m}^{2}$, a waist circumference of $80.7 \pm 8.5 \mathrm{~cm}$, and a hip circumference of $86.8 \pm 8.7 \mathrm{~cm}$. Among women these measures were $23.1 \pm 3.2 \mathrm{~kg} / \mathrm{m}^{2}, 72.4 \pm 7.0 \mathrm{~cm}$, and $81.4 \pm 6.7 \mathrm{~cm}$, respectively.

Figure 3.1 shows the percentages active-sedentary, sedentary, and active minutes for each activity. During the activity 'controlled sitting' the accelerometer recorded, as expected, predominantly sedentary minutes. The activity 'controlled fidgeting' resulted in active-sedentary minutes, but $>50 \%$ of the time were active minutes. The other sedentary activities resulted predominantly in sedentary minutes. However, during the activities 'watching TV', 'playing cards', and 'preparing food' also active-sedentary and active minutes were recorded.

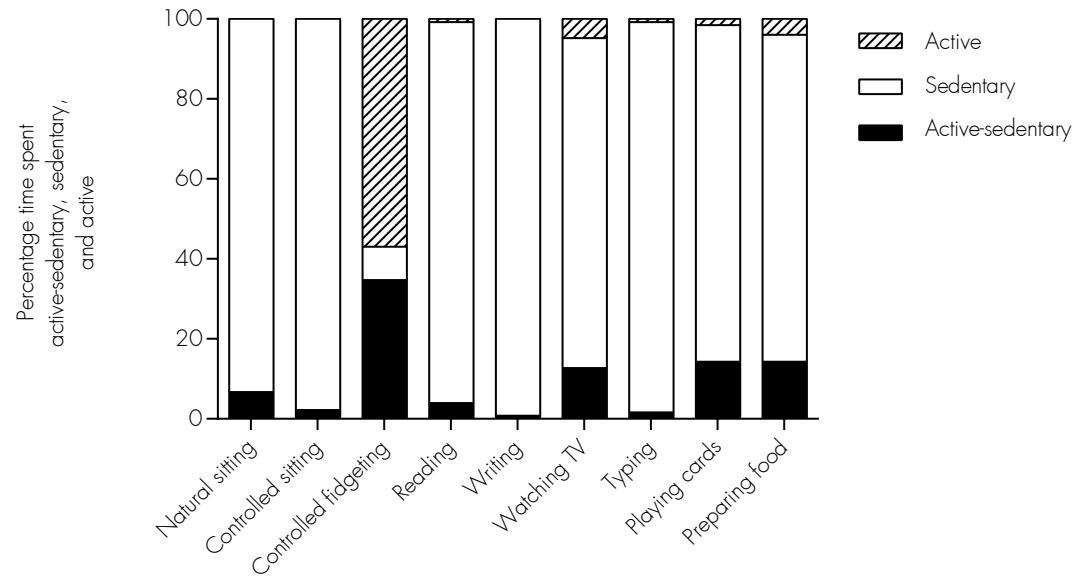

Figure 3.1: Percentage active-sedentary, sedentary, and active minutes of each sedentary activity. 
Table 3.2 presents the number of participants with one or more active-sedentary and one or more active minutes per activity. During the activities 'controlled fidgeting', 'watching TV', 'playing cards', and 'preparing food' most participants had activesedentary minutes. These numbers were statistically significantly different from the number of participants that recorded active-sedentary minutes during the activity 'natural sitting'. During the activity 'controlled fidgeting', the majority of participants recorded one or more active minutes and $22 \%$ of the participants had also active minutes while watching TV and preparing food. These numbers were statistically significantly different from the number of participants that recorded active minutes during the activity 'natural sitting'.

Table 3.2: Number of participants with one or more active-sedentary minutes and one or more active minutes per sedentary activity

\begin{tabular}{|c|c|c|c|c|c|c|c|c|c|}
\hline & $\begin{array}{l}\text { Natural } \\
\text { sitting }\end{array}$ & $\begin{array}{l}\text { Controlled } \\
\text { sitting }\end{array}$ & $\begin{array}{l}\text { Controlled } \\
\text { fidgeting }\end{array}$ & Reading & Writing & $\begin{array}{l}\text { Watching } \\
\text { TV }\end{array}$ & Typing & $\begin{array}{l}\text { Playing } \\
\text { cards }\end{array}$ & $\begin{array}{l}\text { Preparing } \\
\text { food }\end{array}$ \\
\hline \multicolumn{10}{|c|}{ Active-sedentary minutes } \\
\hline$n(\%)$ & $3(16.7)$ & $2(11.1)$ & $12(66.7)$ & $4(22.2)$ & $1(5.6)$ & 12 (66.7) & $2(11.1)$ & $10(55.6)$ & $10(55.6)$ \\
\hline P-value* & ref & 1.000 & 0.004 & 1.000 & 0.625 & 0.012 & 1.000 & 0.065 & 0.016 \\
\hline \multicolumn{10}{|c|}{ Active minutes } \\
\hline$n(\%)$ & $0(0)$ & $0(0)$ & $16(88.9)$ & $1(5.6)$ & $0(0)$ & $4(22.2)$ & $1(5.6)$ & $2(11.1)$ & $4(22.2)$ \\
\hline P-value* & ref & - & $<0.001$ & 1.000 & - & 0.125 & 1.000 & 0.500 & 0.125 \\
\hline
\end{tabular}

${ }^{*}$ Comparison of the number of participants with active-sedentary/active minutes between each sedentary activity and 'natural sitting'.

Table 3.3 shows descriptive characteristics of the average cpm per activity for each axis. The maximum average cpm in the $\mathrm{V}$ axis was for all activities except 'controlled fidgeting' $<100 \mathrm{cpm}$, while in the AP or the ML axes this was $>100 \mathrm{cpm}$ for the activities 'natural sitting', 'watching TV', 'playing cards', 'preparing food', and 'controlled fidgeting'. The mean and median of the average cpm were for all activities in all axes $<100 \mathrm{cpm}$, except for the activity 'controlled fidgeting' which showed in all axes a mean and median $>100 \mathrm{cpm}$. 
Table 3.3: Descriptive characteristics of the average counts per minute in each axis for each sedentary activity

\begin{tabular}{|c|c|c|c|c|}
\hline & & $V$ axis & AP axis & ML axis \\
\hline \multirow{3}{*}{$\begin{array}{l}\text { Natural } \\
\text { sitting }\end{array}$} & range (min - max) & $0.00-39.80$ & $0.00-87.20$ & $0.00-115.00$ \\
\hline & mean $(S D)$ & $6.74(11.14)$ & 12.41 (22.59) & $14.00(28.94)$ \\
\hline & median [Q1 - Q3] & $0.30[0.00-12.05]$ & $2.60[0.45-14.50]$ & $0.00[0.00-19.65]$ \\
\hline \multirow{3}{*}{$\begin{array}{l}\text { Controlled } \\
\text { sitting }\end{array}$} & range (min - max) & $0.00-11.80$ & $0.00-24.20$ & $0.00-14.40$ \\
\hline & mean $(S D)$ & $1.96(3.86)$ & $4.40(8.50)$ & $2.07(4.38)$ \\
\hline & median [Q1 - Q3] & $0.00[0.00-1.75]$ & $0.00[0.00-3.55]$ & $0.00[0.00-1.40]$ \\
\hline \multirow{3}{*}{$\begin{array}{l}\text { Controlled } \\
\text { fidgeting }\end{array}$} & range (min - $\max )$ & $0.00-2607.25$ & $2.25-4777.50$ & $0.00-6508.25$ \\
\hline & mean $(S D)$ & 625.07 (613.76) & $1766.51(1080.12)$ & 2048.38 (1769.87) \\
\hline & median [Q1 - Q3] & $\begin{array}{l}516.88 \\
{[210.44-795.44]}\end{array}$ & $\begin{array}{l}1630.25 \\
{[1184.56-2248.50]}\end{array}$ & $\begin{array}{l}1679.13 \\
{[1008.25-2470.31]}\end{array}$ \\
\hline \multirow[t]{3}{*}{ Reading } & range (min - max) & $0.00-25.14$ & $0.00-52.43$ & $0.00-58.71$ \\
\hline & mean $(S D)$ & $3.66(6.70)$ & $12.41(14.81)$ & $9.40(15.82)$ \\
\hline & median [Q1 - Q3] & $0.29[0.00-5.07]$ & 5.71 [0.64 - 19.39] & $2.64[0.75-8.86]$ \\
\hline \multirow[t]{3}{*}{ Writing } & range (min - max) & $0.00-0.86$ & $0.00-39.71$ & $0.00-7.14$ \\
\hline & mean $(\mathrm{SD})$ & $0.08(0.22)$ & $3.33(9.24)$ & $1.04(2.00)$ \\
\hline & median [Q1 - Q3] & $0.00[0.00-0.00]$ & $0.07[0.00-3.00]$ & $0.00[0.00-1.39]$ \\
\hline \multirow{3}{*}{$\begin{array}{l}\text { Watching } \\
\text { TV }\end{array}$} & range (min - max) & $0.00-49.71$ & $1.29-150.43$ & $0.00-149.14$ \\
\hline & mean $(S D)$ & $17.71(16.01)$ & $39.26(34.23)$ & $37.40(42.37)$ \\
\hline & median [Q1 - Q3] & 14.14 [6.14 - 24.39] & 31.00 [19.93-46.86] & $24.71[10.46-51.32]$ \\
\hline \multirow[t]{3}{*}{ Typing } & range (min - max) & $0.00-14.57$ & $0.00-62.14$ & $0.00-22.14$ \\
\hline & mean $(S D)$ & $1.67(3.75)$ & $6.05(14.64)$ & $5.87(8.04)$ \\
\hline & median [Q1 - Q3] & $0.00[0.00-2.39]$ & $0.86[0.00-5.21]$ & $1.29[0.00-9.82]$ \\
\hline \multirow{3}{*}{$\begin{array}{l}\text { Playing } \\
\text { cards }\end{array}$} & range (min - max) & $0.00-31.00$ & $0.86-236.14$ & $0.00-72.00$ \\
\hline & mean $(S D)$ & $6.93(9.00)$ & $51.37(53.87)$ & $23.31(21.05)$ \\
\hline & median [Q1 - Q3] & $3.50[0.00-13.21]$ & $42.43[16.46-65.07]$ & $20.50[6.46-35.79]$ \\
\hline \multirow{3}{*}{$\begin{array}{l}\text { Preparing } \\
\text { food }\end{array}$} & range (min - max) & $0.00-50.43$ & $0.00-163.00$ & $0.00-139.29$ \\
\hline & mean $(S D)$ & $10.96(16.35)$ & $32.86(44.04)$ & $33.79(36.38)$ \\
\hline & median [Q1 - Q3] & $2.07[0.00-15.75]$ & $13.86[7.68-34.5]$ & $23.64[7.11-53.43]$ \\
\hline
\end{tabular}

$V$, vertical; $A P$, anteroposterior; $M L$, mediolateral. 


\section{DISCUSSION}

In this experimental study we examined whether a hip-worn tri-axial accelerometer recorded movement in the AP and $M L$ axes during common sedentary activities. We demonstrated that activity counts at an intensity level $\geq 100 \mathrm{cpm}$ in the AP and/or ML axes were recorded while participants were in a sedentary position. Thus, common sedentary activities such as 'playing cards' and 'preparing food' consisted not only of sedentary time $(<100 \mathrm{cpm}$ in the $V$ axis), but of active-sedentary time as well $1<100 \mathrm{cpm}$ in the $V$ axis and $\geq 100 \mathrm{cpm}$ in the AP or ML axes). Further, also some active time ( $\geq 100 \mathrm{cpm}$ in all axes) was recorded. Since during all examined sedentary activities in this experiment some active-sedentary time was recorded, we recommend to include data of the AP and ML axes in sedentary behaviour studies in order to distinguish those with more movement during sedentary time from those with less movement. This may be of interest as the movement has a level of intensity that is relevant for health.

The results of Bankoski's et al. study ${ }^{10}$ were a first indication that the active-sedentary time indeed could be relevant. In this study a higher degree of activity during sedentary time, which was based on $\mathrm{V}$ axis data of a hip-worn accelerometer, has been associated with a decreased likelihood of the metabolic syndrome. In this line, experimental studies on non-exercise activity have shown that fidgeting-like movement is associated with substantial increases in energy expenditure and is inversely related to body weight and body weight gain. ${ }^{11-13}$ In addition, it has recently been reported that self-reported fidgeting modified the association between self-reported sitting time and mortality. ${ }^{14}$ Although fidgeting and sitting time were based on self-reported measures in this study, it has been hypothesized that movement involved in fidgeting may protect against detrimental effects of sedentary behaviour. ${ }^{14}$ Taken together, these studies suggest that active-sedentary time may be meaningful for health. To further investigate this notion, population-based studies that use tri-axial accelerometry data to examine associations of active-sedentariness with health outcomes are needed.

Since we demonstrated that during sedentary activities, a hip-worn tri-axial accelerometer recorded not only $>100 \mathrm{cpm}$ in the AP and ML axes, but in the $\mathrm{V}$ axis as well, it can be discussed whether a cut-off point of $<100 \mathrm{cpm}$ in the $V$ axis should be used to identify sedentary time. Although this cut-off point has been widely used and has also been consistently associated with health outcomes, ${ }^{6-9}$ evidence for the accuracy of cut-off points for sedentary time is limited. To date, two studies recommended a cut-off point of 
$\leq 150 \mathrm{cpm}$ for adults, ${ }^{15,16}$ while two others argued for $\leq 50 \mathrm{cpm}$ for adults, ${ }^{17}$ and $<25$ cpm for older adults. ${ }^{18}$ The most accurate assessment of sedentary behaviour, however, has been demonstrated when posture-based devices were used. ${ }^{15,19}$ Since these devices discriminate between sedentary and upright positions, misclassification of standing as sedentary time is prevented, so estimations of sedentary time based on posture data are more accurate than estimations based on acceleration data. ${ }^{15,19}$ However, information on intensity levels of movement during sedentary time is lacking when using solely posture-based measurement. Therefore, future studies ideally should use a combination of posture- and acceleration-based measurement which provides an accurate and comprehensive assessment of sedentary behaviour.

To further verify the presence of active-sedentary time, studies are needed to examine whether our results can be reproduced. Our study was conducted in a small study population consisting of young adults with a normal weight; therefore studies in other populations, such as middle-aged and older adults, and overweight and obese participants are required. Furthermore, it will be necessary to examine whether our results can be reproduced outside the laboratory setting. However, we have demonstrated that during sedentary activities, activity counts with an intensity level $\geq 100 \mathrm{cpm}$ were recorded. Since this would be classified as physical activity outside the laboratory setting, ${ }^{2}$ it is recommended to include posture-based measurement for the accurate assessment of sedentary time. Subsequently, examining intensity levels of active-sedentary time and its associations with health outcomes is necessary and of interest, especially for those who are unable to participate in physical activity. 


\section{REFERENCES}

1. Sedentary Behaviour Research Network. Standardized use of the terms "sedentary" and "sedentary behaviours." Appl Physiol Nutr Metab. 2012;37:540-2.

2. Freedson PS, Melanson E, Sirard J. Calibration of the computer science and applications, inc. accelerometer. Med Sci Sport Exerc. 1998;30(5):777-81.

3. Matthews CE, Chen KY, Freedson PS, Buchowski MS, Beech M, Pate RR, et al. Amount of time spent in sedentary behaviors in the United States, 2003-2004. Am J Epidemiol. 2008; 167(7):875-81.

4. Davis MG, Fox KR, Hillsdon M, Sharp DJ, Coulson JC, Thompson JL. Objectively measured physical activity in a diverse sample of older urban UK adults. Med Sci Sports Exerc. $2011 ; 43(4): 647-54$.

5. Arnardottir NY, Koster A, Van Domelen DR, Brychta RJ, Caserotti P, Eiriksdottir G, et al. Objective measurements of daily physical activity patterns and sedentary behaviour in older adults: Age, Gene/Environment Susceptibility-Reykjavik Study. Age Ageing. 2012;0:1-7.

6. Brocklebank LA, Falconer CL, Page AS, Perry R, Cooper AR. Accelerometer-measured sedentary time and cardiometabolic biomarkers: A systematic review. Prev Med. 2015;76:92-102.

7. Edwardson CL, Gorely T, Davies M, Gray $L$, Khunti K, Wilmot EG, et al. Association of sedentary behaviour with metabolic syndrome: A meta-analysis. PLoS One. 2012;7(4):e34916.

8. Wilmot EG, Edwardson CL, Achana FA, Davies M, Gorely T, Gray L, et al. Sedentary time in adults and the association with diabetes, cardiovascular disease and death: Systematic review and meta-analysis. Diabetologia. 2012;55(11):2895-905.

9. Biswas A, Oh PI, Faulkner GE, Bajaj RR, Silver MA, Mitchell MS, et al. Sedentary time and its association with risk for disease incidence, mortality, and hospitalization in adults. Ann Intern Med. 2015;162(10):123-32.
10. Bankoski A, Harris TB, McClain JJ, Brychta RJ, Caserotti P, Chen KY, et al. Sedentary activity associated with metabolic syndrome independent of physical activity. Diabetes Care. $2011 ; 34(2): 497-503$

11. Johanssen D, Ravussin E. Spontaneaous physical activity: Relationship between figeting and body weight control. Curr Opin Endocrinol Diabetes Obes. 2008;15(5):409-15.

12. Levine JA, Schleusner SJ, Jensen MD. Energy expenditure of nonexercise activity. Am J Clin Nutr. 2000;72(6):1451-4.

13. Levine JA. Nonexercise activity thermogenesis (NEAT): Environment and biology. Am J Physiol Endocrinol Metab. 2004;286(5):E675-85

14. Hagger-Johnson G, Gow AJ, Burley V, Greenwood D, Cade JE. Sitting time, fidgeting, and all-cause mortality in the UK Women's Cohort Study. Am J Prev Med. 2016;50(2):154-60.

15. Kozey-Keadle S, Libertine A, Lyden K, Staudenmayer J, Freedson PS. Validation of wearable monitors for assessing sedentary behavior. Med Sci Sport Exerc. $2011 ; 43(8): 1561-7$.

16. Carr L, Mahar MT. Accuracy of intensity and inclinometer output of three activity monitors for identification of sedentary behavior and lightintensity activity. J Obes. 2012;2012:1-9.

17. Crouter SE, DellaValle DM, Haas JD, Frongillo EA, Bassett DR. Validity of ActiGraph 2-regression model and Matthews and NHANES and cut-points for assessing free-living physical activity. J Phys Act Heal. 2013;10(4):504-14.

18. Aguilar-Farías N, Brown WJ, Peeters GMEE. ActiGraph GT3X+ cut-points for identifying sedentary behaviour in older adults in free-living environments. J Sci Med Sport. 2014;17(3):293-9.

19. Godfrey A, Culhane KM, Lyons GM. Comparison of the performance of the activPAL $L^{T M}$ Professional physical activity logger to a discrete accelerometer-based activity monitor. Med Eng Phys. $2007 ; 29(8): 930-4$. 
Chapt

照 


\section{$\underline{\text { AB STRACT }}$}

Background: Sedentary behaviour is associated with adverse health effects. Insights into associated determinants are essential to prevent sedentary behaviour and limit health risks. Sedentary behaviour should be viewed as a distinct health behaviour; therefore, its determinants should be independently identified.

Purpose: This study examines the prospective associations between a wide range of midlife determinants and objectively measured sedentary time in old age.

Methods: Data from 565 participants (age 73-92 years) of the AGESII-Reykjavik Study were used. Participants wore an accelerometer (ActiGraph GT3X) on the right hip for seven consecutive days. On average, 31 years earlier (during midlife), demographic, socioeconomic, lifestyle, and biomedical factors were collected. Linear regression models were used to examine prospective associations between midlife determinants and sedentary time ( $<100$ counts per minute) in old age.

Results: After adjustment for sex, age, follow-up time, minutes of moderate to vigorous physical activity, body mass index, health status, mobility limitation, and joint pain in old age, the midlife determinants not being married, primary education, living in a duplex or living in an apartment (vs villa), being obese, and having a heart disease were associated with, on average, 15.3, 12.4, $13.5,13.3,21.8$, and 38.9 sedentary minutes more per day in old age, respectively.

Conclusions: This study shows that demographic, socioeconomic, and biomedical determinants in midlife were associated with considerably more sedentary time per day in old age. These results can indicate the possibility of predicting sedentariness in old age, which could be used to identify target groups for prevention programs reducing sedentary time in older adults. 


\section{INTRODUCTION}

During the last years there has been growing interest in sedentary behaviour as a risk factor for adverse health effects, independent of physical activity. Sedentary behaviour, such as sitting, lying down, watching TV and using the computer, has been associated with mortality ${ }^{1-5}$ and metabolic and cardiovascular risk factors. ${ }^{6-11}$

Sedentary time increases with age and older adults spend up to $80 \%$ of their waking time being sedentary. ${ }^{12-15}$ It is important to identify determinants that contribute to a (highly) sedentary lifestyle among older adults to prevent this lifestyle and limits its health effects.

Determinants of physical activity and, in particular, moderate to vigorous physical activity (MVPA), such as health status and self-efficacy, have been studied extensively. ${ }^{16}$ However, sedentary behaviour should be viewed as a distinct health behaviour which differs from a lack of MVPA, and therefore its determinants should be independently identified. To date, only a few studies have prospectively examined the determinants of self-reported ${ }^{17,18}$ or objectively measured sedentary time. ${ }^{19,20}$ The number of determinants investigated in these studies were limited and the study populations were middle aged.

To develop prevention programs for sedentary behaviour, it is of importance to study a broad spectrum of determinants over the lifetime that affects sedentary time. Therefore, this study examines over a period of three decades the prospective associations between a wide-range of midlife determinants (demographic, socioeconomic, lifestyle, biomedical) and objectively measured sedentary time in old age, in a subsample of the Age, Gene/ Environment Susceptibility (AGES)-Reykjavik Study.

\section{METHODS}

\section{Study population}

For this study a subsample of the Age, Gene/Environment Susceptibility (AGES)-Reykjavik Study cohort was used. The AGES-Reykjavik Study originates from the Reykjavik Study, which was established in 1967 and comprised a random sample of 30,795 participants born in 1907-1935 and residing in Reykjavik, Iceland. ${ }^{21}$ Measurements for the Reykjavik Study were conducted in the period between 1967 and 1992. From 2002 until 2006 the measurements for the AGES-Reykjavik Study took place in 5,764 participants of the 
original cohort. ${ }^{21}$ Measurements for the follow-up AGESII-Reykjavik Study took place in 2007-2011 among 3,411 participants. From April 2009 to June 2010, an accelerometry substudy was performed in which 658 participants who did not have severe cognitive dysfunction (Mini-Mental State Examination $\leq 20$ ) were asked to wear an accelerometer for seven consecutive days. ${ }^{12}$ After excluding participants who did not record at least one valid day (at least $10 \mathrm{~h}$ of monitoring) ( $n=15)$ or missing data of the measurements during the Reykjavik Study, AGES-Reykjavik Study or AGESII-Reykjavik Study ( $n=78$ ), a total of 565 participants (aged 73 to 92 years) were included in the current analyses (Figure 4.1). All participants gave written informed consent.

The AGES Reykjavik Study was approved by the institutional review boards of the National Institute on Aging, the National Bioethics Committee (VSN: 00-063) and the Data Protection Authority.

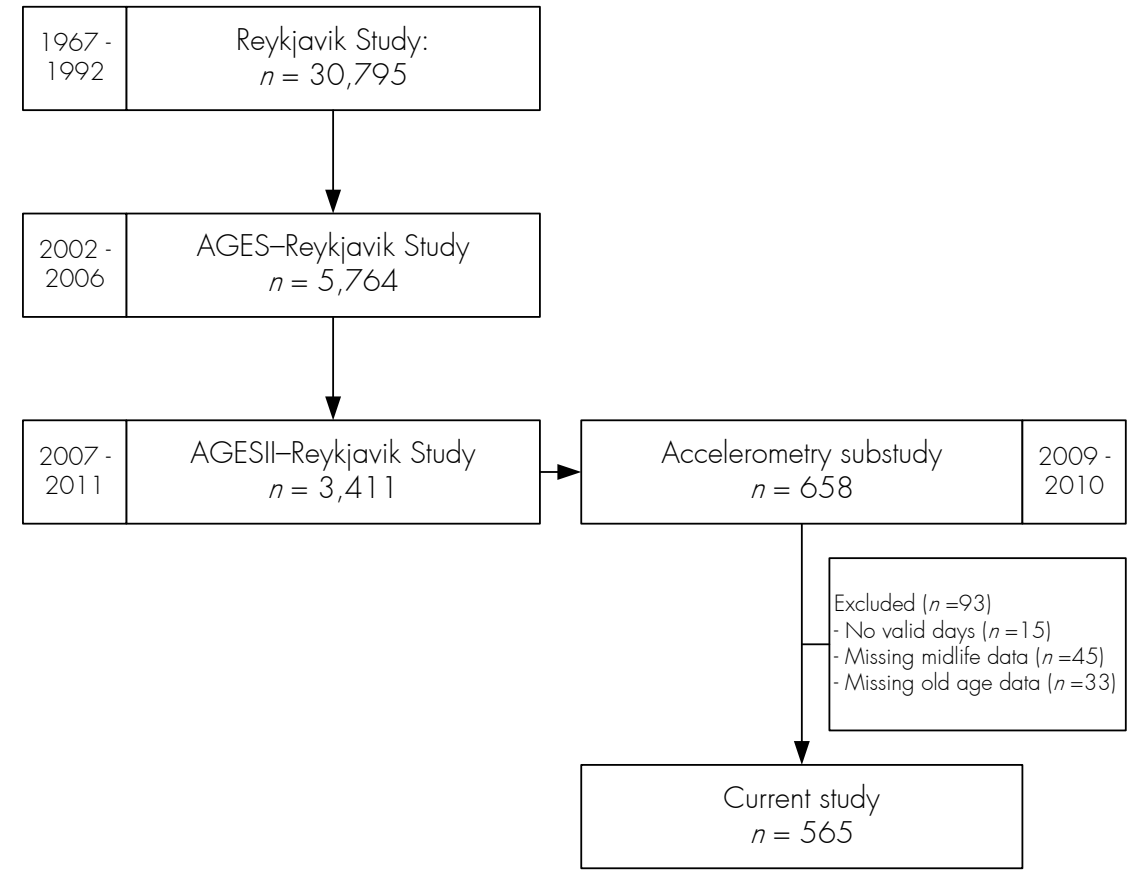

Figure 4.1: Flow of participants. 


\section{Measurements}

Sedentary time in old age

Sedentary time was assessed using an accelerometer (ActiGraph GT3X, Ft. Walton Beach, FL, USA), which was attached to a belt and was worn on the right hip. Participants were instructed to wear the accelerometer for seven consecutive days and only remove the monitor before going to bed and during showering, bathing, swimming and other water activities. The triaxial ActiGraph recorded movement on the vertical, anteroposterior and mediolateral axes. Raw accelerometry data were processed and averaged by minute using customized software written in MATLAB R2006a (The MathWorks; Natick MA, USA) to obtain relevant outcome variables. Non-wear time was defined as any interval $\geq 60$ consecutive min of zero counts in all three axes, in which a period up to $2 \mathrm{~min}$ of nonzero counts under 100 in the vertical axis was allowed. ${ }^{12}$ A valid day was defined as at least $10 \mathrm{~h}$ of monitoring. The outcome measure used in this analysis was the percentage of wear time minutes per day spent sedentary (0-99 counts per minute (cpm) in the vertical axis).

\section{Midlife determinants}

A variety of midlife determinants collected as part of the Reykjavik Study were used for the analysis, including demographic characteristics, socioeconomic factors, lifestyle factors, and biomedical factors. Data were collected by questionnaires (detailed medical history, health-related behaviour), physical examinations, and laboratory tests.

Demographic characteristics of sex, age and marital status were derived from a questionnaire. Marital status was categorized as married/not married, with the categories single, widow(er), divorced and separated, collapsed into not married, because of the small numbers in these categories. Due to the large number of individuals without this information ( $n=178$ ), this group was included as separate category.

Socioeconomic factors included level of education, housing type, and occupation, which were derived from a questionnaire. Level of education was categorized into college/university, secondary and primary. Housing type was categorized into villa, duplex, and apartment, and occupation was categorized into professional work, light work, manual labour, and homemaker.

Lifestyle factors included smoking status, physical activity, active commuting, and occupation activity and were derived from a questionnaire. These factors were categorized into never smoker, previous smoker and current smoker; active/inactive; active commuting/not active commuting; and for occupational activity "on the move", standing, and sitting respectively. 
Biomedical factors included body mass index (BMI), weight status, hypertension, cholesterol levels, triglyceride levels, heart disease, type 2 diabetes, arthritis, lung disease and lung function (FVC, FEV1, FEV1/FVC ratio). Height and weight were measured during the physical examinations and BMI was calculated as kilogram per meter squared. Weight status was categorized as $\mathrm{BMI}<25$, BMI 25-30 and $\mathrm{BMI} \geq 30$. Hypertension was defined as self-reported hypertension and/or a systolic blood pressure $\geq 140 \mathrm{mmHg}$ and/ or a diastolic blood pressure $\geq 90 \mathrm{mmHg} .{ }^{22}$ Cholesterol and triglyceride levels in blood were obtained by laboratory tests. The presence of heart disease, diabetes, arthritis, and lung disease were derived from a questionnaire about whether participants had ever sought a doctor or were treated in a hospital for a disease. Lung function was obtained by spirometry. The spirometric reference values were calculated using the NHANES III/ Hankinson equations, ${ }^{23}$ and the FEV1/FVC ratio was calculated using the observed values.

\section{Covariates}

Covariates were measured by means of questionnaires and accelerometry in old age as part of the AGESII-Reykjavik Study and included sex, age, follow-up time, daily minutes of MVPA (MVPA $\geq 2020 \mathrm{cpm}$ ), BMl, health status, mobility limitation, and joint pain. Follow-up time was calculated as the time between midlife measurements and AGESII measurements. Health status was defined as the number of the following conditions: cancer (measured as part of AGES-Reykjavik Study), arthritis, osteoporosis, Parkinson's disease, heart disease, asthma, chronic obstructive pulmonary disease (COPD), and depression. Mobility limitation was defined as any difficulty walking $500 \mathrm{~m}$ or climbing 10 steps without resting. Joint pain was defined as pain, aching or stiffness in one or more of the following joints: back (upper, middle, under), hips, knees, ankles, and toes.

\section{Statistical analysis}

Descriptive characteristics were summarized as mean and SD or percentages. Chi-square tests and ANOVA were used to examine differences between men and women in the categorical and continuous midlife determinants. Sex differences in the accelerometry variables were examined using ANOVA. Linear regression analysis was used to study whether the midlife determinants predicted the amount of sedentary time in old age. For each determinant the analyses were run separately, with the determinant as the exposure variable and the percentage of minutes per day spent sedentary as the outcome. 
All models were adjusted for sex, age and follow-up time (model 1). Model 2 was additionally adjusted for MVPA (min/day) to understand whether the midlife determinants were related to sedentary time in old age independent of MVPA. Model 3 was additionally adjusted for BMI, health status, mobility limitation, and joint pain in old age to exclude the effects of these conditions on sedentary time. Interactions of all midlife determinants with gender were not statistically significant $(P>0.10)$, and therefore, the main analyses were conducted for men and women together. All analyses were conducted with IBM SPSS Statistics 20.0. Significance level was set at $<0.05$.

\section{RESULTS}

Descriptive characteristics of the study population are presented in Table 4.1. In midlife, women were significantly older than men and were less often married. Women also had a lower educational level, lived more often in an apartment, were less often employed and reported more being "on the move" during work than men. Women smoked less than men, had a lower BMI, were less overweight, had less hypertension and lower triglyceride levels (all $P<0.05$ ). Self-reported physical activity and active commuting did not significantly differ between men and women.

Table 4.2 shows the physical activity characteristics assessed by accelerometry of the participants in old age (mean age was $80.0 \pm 4.7$ years). Almost $50 \%$ of the participants $(49.9 \%)$ provided seven valid days of data (>10 h of monitoring), with $28.5 \%$ providing 6 days; $9.6 \% 5$ days; $4.1 \% 4$ days; and $3.9 \% 3$ days. Participants spent most of their wear time $(75 \%)$ being sedentary, over $10 \mathrm{~h}$ per day in both sexes. Men were significantly more sedentary than women, but recorded also more minutes in MVPA. 
Table 4.1: Descriptive characteristics of the study population in midlife

\begin{tabular}{|c|c|c|c|c|c|c|c|c|}
\hline \multicolumn{2}{|l|}{ Determinants } & \multicolumn{2}{|c|}{ Total } & \multicolumn{2}{|c|}{ Men } & \multicolumn{2}{|c|}{ Women } & \multirow[t]{2}{*}{ P-value } \\
\hline \multirow[t]{3}{*}{ Demographic } & $\operatorname{Sex}(n)$ & 565 & & 222 & $(39.3 \%)$ & 343 & $(60.7 \%)$ & \\
\hline & Age (years) & 48.8 & $(6.2)$ & 46.9 & (5.2) & 50.0 & (6.4) & $<0.001$ \\
\hline & $\begin{array}{l}\text { Marital status (\%) } \\
\text { - married } \\
\text { - not married } \\
\text { - missing }\end{array}$ & $\begin{array}{r}60.7 \\
7.8 \\
31.5\end{array}$ & & $\begin{array}{r}81.1 \\
5.9 \\
13.0\end{array}$ & & $\begin{array}{r}47.5 \\
9.0 \\
43.5\end{array}$ & & $<0.001$ \\
\hline \multirow[t]{3}{*}{$\begin{array}{l}\text { Socio- } \\
\text { economic }\end{array}$} & $\begin{array}{l}\text { Level of education (\%) } \\
\text { - college/university } \\
\text { - secondary } \\
\text { - primary }\end{array}$ & $\begin{array}{l}20.5 \\
50.1 \\
29.4\end{array}$ & & $\begin{array}{l}31.1 \\
50.0 \\
18.9\end{array}$ & & $\begin{array}{l}13.7 \\
50.1 \\
36.2\end{array}$ & & $<0.001$ \\
\hline & $\begin{array}{l}\text { Housing type }(\%) \\
\text { - villa } \\
\text { - duplex } \\
\text { - apartment }\end{array}$ & $\begin{array}{l}33.6 \\
21.1 \\
45.3\end{array}$ & & $\begin{array}{l}36.9 \\
24.8 \\
38.3\end{array}$ & & $\begin{array}{l}31.5 \\
18.7 \\
49.8\end{array}$ & & 0.023 \\
\hline & $\begin{array}{l}\text { Occupation group (\%) } \\
\text { - professional work } \\
\text { - light work } \\
\text { - manual labour } \\
\text { - homemaker }\end{array}$ & $\begin{array}{l}17.9 \\
24.4 \\
14.5 \\
43.2 \\
\end{array}$ & & $\begin{array}{r}36.0 \\
27.9 \\
33.3 \\
2.8 \\
\end{array}$ & & $\begin{array}{r}6.1 \\
22.2 \\
2.3 \\
69.4 \\
\end{array}$ & & $<0.001$ \\
\hline \multirow[t]{4}{*}{ Lifestyle } & $\begin{array}{l}\text { Smoking status (\%) } \\
\text { - never smoker } \\
\text { - previous smoker } \\
\text { - current smoker }\end{array}$ & $\begin{array}{l}44.3 \\
19.6 \\
36.1\end{array}$ & & $\begin{array}{l}32.4 \\
21.2 \\
46.4\end{array}$ & & $\begin{array}{l}51.9 \\
18.7 \\
29.4\end{array}$ & & $<0.001$ \\
\hline & $\begin{array}{l}\text { Physical activity } \\
\text { (\%active) }\end{array}$ & 28.0 & & 27.9 & & 28.0 & & 0.988 \\
\hline & $\begin{array}{l}\text { Active commuting } \\
\text { (\%active) }\end{array}$ & 11.3 & & 8.1 & & 13.4 & & 0.052 \\
\hline & $\begin{array}{l}\text { Occupation activity (\%) } \\
\text { - on the move } \\
\text { - standing } \\
\text { - sitting }\end{array}$ & $\begin{array}{r}65.1 \\
7.4 \\
27.5 \\
\end{array}$ & & $\begin{array}{l}40.1 \\
16.2 \\
43.7 \\
\end{array}$ & & $\begin{array}{r}81.3 \\
1.7 \\
16.9 \\
\end{array}$ & & $<0.001$ \\
\hline \multirow[t]{12}{*}{ Biomedical } & BMI $\left(\mathrm{kg} / \mathrm{m}^{2}\right)$ & 25.0 & (3.5) & 25.4 & (3.3) & 24.7 & (3.6) & 0.009 \\
\hline & $\begin{array}{l}\text { Weight status (\%) } \\
\text { - } \mathrm{BMI}<25 \\
\cdot 25 \leq \mathrm{BMI}<30 \\
\text { - } \mathrm{BMI} \geq 30\end{array}$ & $\begin{array}{r}55.9 \\
35.4 \\
8.7\end{array}$ & & $\begin{array}{r}47.3 \\
45.0 \\
7.7\end{array}$ & & $\begin{array}{r}61.5 \\
29.2 \\
9.3\end{array}$ & & 0.001 \\
\hline & Hypertension (\%) & 30.3 & & 44.6 & & 21.0 & & $<0.001$ \\
\hline & $\begin{array}{l}\text { Cholesterol } \\
(\%>5.0 \mathrm{mmol} / \mathrm{L})\end{array}$ & 88.1 & & 89.2 & & 87.5 & & 0.535 \\
\hline & $\begin{array}{l}\text { Triglyceride } \\
(\%>1.7 \mathrm{mmol} / \mathrm{L})\end{array}$ & 10.6 & & 18.5 & & 5.5 & & $<0.001$ \\
\hline & Heart disease (\%) & 1.1 & & 0.5 & & 1.5 & & 0.254 \\
\hline & Diabetes type $2(\%)$ & 0.5 & & 0.5 & & 0.6 & & 0.832 \\
\hline & Arthritis (\%) & 0.5 & & 0.5 & & 0.6 & & 0.832 \\
\hline & Lung disease (\%) & 1.4 & & 0.9 & & 1.7 & & 0.405 \\
\hline & $\begin{array}{l}\text { Lung function (\%) } \\
\text { - FVC (observed }<80 \% \\
\text { predicted) }\end{array}$ & 20.0 & & 18.0 & & 21.3 & & 0.343 \\
\hline & $\begin{array}{l}\text { - FEV } 1 \text { (observed }<80 \% \\
\text { predicted) }\end{array}$ & 20.9 & & 18.5 & & 22.4 & & 0.256 \\
\hline & - FEV $1 /$ FVC $(<0.7)$ & 10.4 & & 9.5 & & 11.1 & & 0.539 \\
\hline
\end{tabular}

Values are mean $(S D)$, unless stated otherwise. BMI, body mass index. 
Table 4.2: Physical activity characteristics of the study population in old age

\begin{tabular}{|lrlrlrlr|} 
& \multicolumn{2}{c}{ Total } & \multicolumn{2}{c}{ Men } & \multicolumn{2}{c|}{ Women } & P-value \\
Age (years) & 80.0 & $(4.7)$ & 79.7 & $(4.3)$ & 80.2 & $(5.0)$ & \\
\hline Valid days (n) & 6.0 & $(1.6)$ & 6.0 & $(1.4)$ & 6.0 & $(1.4)$ & 0.828 \\
Wear time (h/day) & 13.7 & $(1.5)$ & 13.9 & $(1.6)$ & 13.6 & $(1.4)$ & 0.034 \\
Daily counts per minute & 128.5 & $(69.8)$ & 132.5 & $(77.0)$ & 126.0 & $(64.8)$ & 0.283 \\
Sedentary time (h/day) & 10.3 & $(1.5)$ & 10.6 & $(1.5)$ & 10.1 & $(1.4)$ & $<0.001$ \\
\% sedentary time per day & 75.3 & & 77.0 & & 74.3 & & $<0.001$ \\
MVPA (min/day) & 6.7 & $(10.0)$ & 9.5 & $(12.8)$ & 4.9 & $(7.2)$ & $<0.001$ \\
\% MVPA per day & 0.8 & & 1.1 & & 0.6 & $<0.001$ \\
\hline
\end{tabular}

Values are mean (SD), unless stated otherwise. MVPA, moderate to vigorous physical activity.

In Table 4.3, the results of the linear regression analyses with the midlife determinants of sedentary time are presented. After adjustment for sex, age, and follow-up time (model 1), level of education, living in an apartment, current smoker status, BMI, obesity $(B M I \geq 30)$, heart disease and lung function (FVC and FEV1) were significantly associated with a higher amount of sedentary time per day in old age. After additionally adjusting for minutes of MVPA (model 2), primary education, living in a duplex or apartment, BMI, obesity, heart disease and FVC remained statistically significant associated with more sedentary time. These variables except BMI and FVC remained significantly associated with a higher percentage of sedentary time after adjusting for BMI, health status, mobility limitation, and joint pain in old age (model 3). Not being married was also significantly associated with more sedentary time in this model.

The percentages of sedentary time (B) shown in Table 4.3 correspond with an average of $15.3(95 \% \mathrm{Cl}=0.3 ; 30.4)$ sedentary minutes more per day in old age for participants who were not married at midlife compared with those who were married; $12.4(0.7 ; 24.0)$ sedentary minutes more for participants with primary education compared with college/ university educated; $13.5(2.6 ; 24.5)$ and $13.3(4.4 ; 22.2)$ sedentary minutes more for participants living in a duplex or apartment, respectively, compared with those living in a villa; $21.8(5.4 ; 38.2)$ sedentary minutes more for obese participants compared with those with $\mathrm{BMI} \leq 25$; and $38.9(0.9 ; 76.9)$ sedentary minutes more for participants with a heart disease.

In additional analyses, weight status and heart disease were further adjusted for marital status, level of education and housing type at midlife; this did not alter the results (data not shown). 
Table 4.3: Associations between midlife determinants and the amount of time spent being sedentary (\% sedentary minutes of wear time) in old age

\begin{tabular}{|c|c|c|c|c|c|c|c|}
\hline \multirow[b]{2}{*}{ Determinants } & & \multicolumn{2}{|c|}{ Model 1} & \multicolumn{2}{|c|}{ Model 2} & \multicolumn{2}{|c|}{ Model 3} \\
\hline & & B & P-value & B & P-value & B & P-value \\
\hline Demographic & $\begin{array}{l}\text { Marital status } \\
\text { - married } \\
\text { - not married } \\
\text { - missing }\end{array}$ & $\begin{array}{r}\text { ref } \\
2.07 \\
0.86\end{array}$ & $\begin{array}{l}0.125 \\
0.457\end{array}$ & $\begin{array}{r}\text { ref } \\
2.39 \\
1.28\end{array}$ & $\begin{array}{l}0.062 \\
0.242\end{array}$ & $\begin{array}{r}\text { ref } \\
2.48 \\
1.08\end{array}$ & $\begin{array}{l}0.046 \\
0.306\end{array}$ \\
\hline \multirow[t]{3}{*}{$\begin{array}{l}\text { Socio- } \\
\text { economic }\end{array}$} & $\begin{array}{l}\text { Level of education } \\
\text { - college/university } \\
\text { - secondary } \\
\text { - primary }\end{array}$ & $\begin{array}{r}\text { ref } \\
1.84 \\
2.43\end{array}$ & $\begin{array}{l}0.048 \\
0.019\end{array}$ & $\begin{array}{l}\text { ref } \\
1.51 \\
2.51\end{array}$ & $\begin{array}{l}0.087 \\
0.011\end{array}$ & $\begin{array}{l}\text { ref } \\
0.90 \\
2.00\end{array}$ & $\begin{array}{l}0.295 \\
0.038\end{array}$ \\
\hline & $\begin{array}{l}\text { Housing type } \\
\text { - villa } \\
\text { - duplex } \\
\text { - apartment }\end{array}$ & $\begin{array}{l}\text { ref } \\
1.56 \\
2.46\end{array}$ & $\begin{array}{l}0.109 \\
0.002\end{array}$ & $\begin{array}{r}\text { ref } \\
2.69 \\
2.67\end{array}$ & $\begin{array}{r}0.004 \\
<0.001\end{array}$ & $\begin{array}{r}\text { ref } \\
2.19 \\
2.16\end{array}$ & $\begin{array}{l}0.016 \\
0.004\end{array}$ \\
\hline & $\begin{array}{l}\text { Occupation group } \\
\text { - professional } \\
\text { - light work } \\
\text { - manual labour } \\
\text { - homemaker }\end{array}$ & $\begin{array}{l}\text { ref } \\
0.75 \\
0.43 \\
0.61\end{array}$ & $\begin{array}{l}0.513 \\
0.728 \\
0.632\end{array}$ & $\begin{array}{l}\text { ref } \\
1.16 \\
1.19 \\
1.01\end{array}$ & $\begin{array}{l}0.288 \\
0.314 \\
0.407\end{array}$ & $\begin{array}{l}\text { ref } \\
0.99 \\
0.87 \\
0.66\end{array}$ & $\begin{array}{l}0.349 \\
0.450 \\
0.574\end{array}$ \\
\hline \multirow[t]{4}{*}{ Lifestyle } & $\begin{array}{l}\text { Smoking status } \\
\text { - never smoker } \\
\text { - previous smoker } \\
\text { - current smoker }\end{array}$ & $\begin{array}{r}\text { ref } \\
0.46 \\
1.95\end{array}$ & $\begin{array}{l}0.626 \\
0.017\end{array}$ & $\begin{array}{r}\text { ref } \\
0.12 \\
1.05\end{array}$ & $\begin{array}{l}0.892 \\
0.178\end{array}$ & $\begin{array}{r}\text { ref } \\
-0.28 \\
0.39\end{array}$ & $\begin{array}{l}0.756 \\
0.613\end{array}$ \\
\hline & Physical activity & -0.68 & 0.384 & -0.39 & 0.604 & -0.20 & 0.784 \\
\hline & Active commuting & -0.23 & 0.837 & 0.27 & 0.800 & 0.25 & 0.804 \\
\hline & $\begin{array}{l}\text { Occupation activity } \\
\text { - on the move } \\
\text { - standing } \\
\text { - sitting }\end{array}$ & $\begin{array}{l}\text { ref } \\
0.50 \\
1.21\end{array}$ & $\begin{array}{l}0.727 \\
0.159\end{array}$ & $\begin{array}{l}\text { ref } \\
0.06 \\
0.69\end{array}$ & $\begin{array}{l}0.965 \\
0.395\end{array}$ & $\begin{array}{l}\text { ref } \\
0.03 \\
0.93\end{array}$ & $\begin{array}{l}0.980 \\
0.236\end{array}$ \\
\hline \multirow[t]{10}{*}{ Biomedical } & BMl $\left(\mathrm{kg} / \mathrm{m}^{2}\right)$ & 0.33 & 0.001 & 0.22 & 0.021 & 0.21 & 0.078 \\
\hline & $\begin{array}{l}\text { Weight status } \\
\text { - } \mathrm{BMI}<25 \\
\text { - } 25 \leq \mathrm{BMI}<30 \\
\text { - } \mathrm{BMI} \geq 30\end{array}$ & $\begin{array}{r}\text { ref } \\
1.14 \\
4.94\end{array}$ & $\begin{array}{r}0.132 \\
<0.001\end{array}$ & $\begin{array}{r}\quad \text { ref } \\
0.41 \\
3.87\end{array}$ & $\begin{array}{l}0.573 \\
0.002\end{array}$ & $\begin{array}{r}\text { ref } \\
0.04 \\
3.53\end{array}$ & $\begin{array}{l}0.955 \\
0.009\end{array}$ \\
\hline & Hypertension & -0.20 & 0.802 & 0.11 & 0.883 & 0.14 & 0.846 \\
\hline & Cholesterol & 0.39 & 0.723 & 0.75 & 0.472 & 0.92 & 0.358 \\
\hline & Triglyceride & 1.23 & 0.292 & 0.81 & 0.463 & 0.77 & 0.474 \\
\hline & Heart disease & 8.22 & 0.016 & 7.55 & 0.020 & 6.30 & 0.045 \\
\hline & Diabetes type 2 & -3.77 & 0.434 & -4.27 & 0.350 & -3.17 & 0.476 \\
\hline & Arthritis & -3.77 & 0.434 & -4.27 & 0.350 & -3.17 & 0.476 \\
\hline & Lung disease & 3.12 & 0.293 & 4.56 & 0.105 & 4.58 & 0.094 \\
\hline & $\begin{array}{l}\text { Lung function } \\
\text { - FVC ( } \% \text { of predicted value) } \\
\text { - FEV1 (\% of predicted value) } \\
\text { - FEV1/FVC }\end{array}$ & $\begin{array}{r}-0.07 \\
-0.05 \\
2.04\end{array}$ & $\begin{array}{l}0.003 \\
0.018 \\
0.631\end{array}$ & $\begin{array}{r}-0.05 \\
-0.04 \\
1.19\end{array}$ & $\begin{array}{l}0.039 \\
0.087 \\
0.767\end{array}$ & $\begin{array}{r}-0.04 \\
-0.02 \\
1.56\end{array}$ & $\begin{array}{l}0.114 \\
0.244 \\
0.689\end{array}$ \\
\hline
\end{tabular}

Regression results are presented as unstandardized coefficients (B). BMl, body mass index. Model 1: adjusted for sex, age and follow-up time; model 2 is additionally adjusted for MVPA (min/day); model 3 is additionally adjusted for BMI, health status, mobility limitation, and joint pain in old age. 


\section{DISCUSSION}

To our knowledge, this is the first study that examined the prospective associations between midlife determinants from four different domains (demographic, socioeconomic, lifestyle and biomedical) and objectively measured sedentary time in old age over a period of three decades. The midlife determinants marital status, level of education, housing type, weight status, and heart disease were statistically significantly associated with a higher amount of sedentary time per day in old age, even after adjusting for sex, age, follow-up time, MVPA, BMI, health status, mobility limitation, and joint pain.

The demographic factor not being married and the socioeconomic factors, primary education, living in a duplex or living in an apartment, were associated with, on average, $15.3,12.4,13.5$ and 13.3 min more sedentary time in old age, respectively. Smoking at midlife was the only lifestyle factor that was associated with sedentary time, but exclusively in the unadjusted model. Spending most working hours sitting was not significantly associated with more sedentary time in old age. Finally, two biomedical factors, being obese and having a heart disease, were associated with, on average, 21.8 and $38.9 \mathrm{~min}$ more sedentary time per day in old age.

To date only a few studies have prospectively examined the association of determinants of objectively measured sedentary time. A study among middle aged participants (mean 54 years) investigated associations between two demographic factors (age and sex), two socioeconomic factors (level of education and employment grade), two lifestyle factors (smoking status and self-reported physical activity), two biomedical factors (BMI and general perceived health) and sedentary time, and found that age and education were associated with sedentary time after 13 years of follow-up. ${ }^{19}$ Ekelund et al. ${ }^{20}$ examined the associations between sedentary time and biomedical factors (body weight, BMI, fat mass, and waist circumference) in middle aged participants (mean 49 years). After 5.6 years of follow-up, body weight, BMl, fat mass, and waist circumference predicted an increase in the amount of sedentary time.

BMI was also described as a determinant of a sedentary lifestyle in a study that measured BMI and a sedentary lifestyle at the ages of $41,44,46$ and 54 years, although sedentariness was not measured objectively in that study. ${ }^{18}$ Obesity was prospectively associated with self-reported time spent watching TV per week in a cohort of middle-aged active employees. ${ }^{24}$ Ding et al. ${ }^{17}$ examined individual, social, and environmental correlates of change in self-reported TV viewing time, which was used as a measure for sedentary 
time. They described associations between education, self-reported physical activity, living in a low-walkable neighbourhood, and TV viewing time after 4 years follow-up in a population aged $20-65$ years.

Recently, several studies have been conducted in which determinants of self-reported physical inactivity were examined. ${ }^{25-28}$ However, sedentary behaviour should be assumed as a distinct health behaviour with its own nature and physiology, which differs from a lack of physical activity and should therefore be studied independently. ${ }^{29-31}$ More studies examining the determinants of sedentary behaviour are warranted in order to better advise the development of public health guidelines and prevention programs, as emerging evidence shows the adverse health consequences of sedentary behaviour. ${ }^{32}$

A major strength of the present study was the objective measurement of sedentary time with accelerometry in a large sample of older adults of which $92 \%$ provided at least four valid days. The amount of sedentary time $(75 \%)$ presented in this study is comparable with the amounts described in other studies with older adults, which also used the same cut-off point to define sedentary time. ${ }^{13-15}$ Using a hip-worn accelerometer to define sedentary time may, however, not accurately separate sitting time from standing time. Another strength of this study was adjustment for important confounders such as health status, mobility limitation, and joint pain in the final analyses, which exclude the possibility that these factors account for or contribute to a higher amount of sedentary time, although this could have resulted in overadjustment, as the confounders may be part of the pathway between the midlife determinants and sedentary time or could be a result of sedentary time.

Furthermore, the longitudinal design of the study with a follow-up time of approximately 31 years is an important strength.

Some limitations of the study should also be considered. First, the midlife data were collected between 1967 and 1992, although this limitation was in part addressed by adjusting all analyses for follow-up time. Further, the determinants could easily have changed during that time period. The measurements in midlife were predominantly collected by means of questionnaires, which could possibly have resulted in misclassification of the lifestyle or biomedical factors. Second, the associations between the biomedical factors and the amount of sedentary time are possibly bidirectional because, for example, obesity leads to a more sedentary lifestyle, but more sedentary time could also increase BMI. In this study sedentary time was measured exclusively in old age; therefore it might be possible that obesity or heart disease resulted from a more sedentary lifestyle. However, an extra analysis in which these factors were additionally adjusted for physical activity in 
midlife (a proxy for sedentary time in midlife) did not change the results (data not shown). Furthermore, studies have shown that BMl at baseline predicted sedentary time at follow-up whereas the reverse association was not established. ${ }^{18,20}$ These findings support the hypothesis that the direction of the association is mainly from the biomedical factors to sedentary time. Third, although this study adjusted for a broad spectrum of confounders, it is possible that some unmeasured confounders, such as psychosocial factors (anxiety, isolation), account for some of the presented associations, which could be explored in further research. Also, this study was conducted in a relatively healthy subsample of the AGESII-Reykjavik Study, and therefore, the results might not be representative for the general older population. Finally, as sedentary time was collected over one week, the data may not truly reflect habitual behaviour.

To conclude, this study shows that midlife determinants across several domains: demographic and socioeconomic factors - not being married, lower educational level, and poorer housing-and biomedical factors, including being obese and having a heart disease, were associated with considerably more sedentary time per day in old age. These results can indicate the possibility of predicting sedentariness in old age, years before this behaviour manifests. This information could be used to identify target groups for prevention programs aimed at reducing sedentary time and decreasing the risk of sedentary-related adverse health effects. 


\section{REFERENCES}

1. Koster A, Caserotti P, Patel K V., Matthews CE, Berrigan D, Van Domelen DR, et al. Association of Sedentary Time with Mortality Independent of Moderate to Vigorous Physical Activity. PLoS One. 2012;7(6):e37696.

2. Matthews C, George S, Moore S, Bowles H, Blair A, Park Y, et al. Amount of time spent in sedentary behaviors and cause-specific mortality in US adults. Am J Clin Nutr. 2012;95:437-45.

3. van der Ploeg HP, Chey T, Korda RJ, Banks E, Bauman A. Sitting time and all-cause mortality risk in 222497 Australian adults. Arch Intern Med. 2012;172(6):494-500.

4. Dunstan DW, Barr ELM, Healy GN, Salmon J, Shaw JE, Balkau B, et al. Television viewing time and mortality: The Australian Diabetes, Obesity and Lifestyle Study (AusDiab). Circulation. 2010;121(3):384-91.

5. Katzmarzyk PT, Church TS, Craig CL, Bouchard C. Sitting time and mortality from all causes, cardiovascular disease, and cancer. Med Sci Sports Exerc. 2009;41(5):998-1005.

6. Wilmot EG, Edwardson CL, Achana FA, Davies M, Gorely T, Gray L, et al. Sedentary time in adults and the association with diabetes, cardiovascular disease and death: Systematic review and meta-analysis. Diabetologia. 2012;55(11):2895-905.

7. Edwardson CL, Gorely T, Davies M, Gray U, Khunti K, Wilmot EG, et al. Association of sedentary behaviour with metabolic syndrome: A meta-analysis. PLoS One. 2012;7(4):e34916.

8. Grøntved A. Television viewing and risk of type 2 diabetes, cardiovascular disease, and all-cause mortality. JAMA. $2011 ; 305(23): 2448-55$.

9. Bankoski A, Harris TB, McClain JJ, Brychta RJ, Caserotti P, Chen KY, et al. Sedentary activity associated with metabolic syndrome independent of physical activity. Diabetes Care. $2011 ; 34(2): 497-503$.

10. Healy GN, Matthews CE, Dunstan DW, Winkler EAH, Owen N. Sedentary time and cardiometabolic biomarkers in US adults: NHANES 2003-06. Eur Heart J. $2011 ; 32(5): 590-7$.
11. Healy G, Wijndaele K, Dunstan D, Shaw J, Salmon J, Zimmet $P$, et al. Objectively measured sedentary time, physical activity, and metabolic risk. Diabetes Care. 2008;31 (2):369-71.

12. Arnardottir NY, Koster A, Van Domelen DR, Brychta RJ, Caserotti P, Eiriksdottir G, et al. Objective measurements of daily physical activity patterns and sedentary behaviour in older adults: Age, Gene/Environment Susceptibility-Reykjavik Study. Age Ageing. 2012;0:1-7.

13. Davis MG, Fox KR, Hillsdon M, Sharp DJ, Coulson JC, Thompson JL. Objectively measured physical activity in a diverse sample of older urban UK adults. Med Sci Sports Exerc. $2011 ; 43(4): 647-54$.

14. Evenson K, Buchner D, Morland K. Objective measurement of physical activity and sedentary behavior among US adults aged 60 years or older. Prev Chronic Dis. $2011 ; 9(2)$ :E26.

15. Matthews CE, Chen KY, Freedson PS, Buchowski MS, Beech M, Pate RR, et al. Amount of time spent in sedentary behaviors in the United States, 2003-2004. Am J Epidemiol. 2008;167(7):875-81.

16. Bauman AE, Reis RS, Sallis JF, Wells JC, Loos RJF, Martin BW. Correlates of physical activity: Why are some people physically active and others not? Lancet. 2012;380(9838):258-71.

17. Ding D, Sugiyama T, Winkler E, Cerin E, Wijndaele K, Owen N. Correlates of change in adults' television viewing time: A fouryear follow-up study. Med Sci Sports Exerc. 2012;44(7):1287-92.

18. Mortensen LH, Siegler IC, Barefoot JC, Grønbaek $M$, Sørensen TIA. Prospective associations between sedentary lifestyle and BMI in midlife. Obesity. 2006; 14(8):1462-71.

19. Hamer M, Kivimaki M, Steptoe A. Longitudinal patterns in physical activity and sedentary behaviour from mid-life to early old age: A substudy of the Whitehall II cohort. J Epidemiol Community Health. 2012;66(12):11 10-5.

20. Ekelund U, Brage S, Besson H, Sharp S, Wareham NJ. Time spent being sedentary and weight gain in healthy adults: Reverse or bidirectional causality? Am J Clin Nutr. 2008;88:612-7. 
21. Harris $T B$, Launer $L$, Eiriksdottir $G$, Kjartansson O, Jonsson P, Sigurdsson G, et al. Age, Gene/ Environment Susceptibility-Reykjavik Study: Multidisciplinary applied phenomics. Am J Epidemiol. 2007;165(9):1076-87.

22. Chobanian $A V$, Bakris GL, Black HR, Cushman WC, Green L a, Izzo JL, et al. Seventh report of the Joint National Committee on prevention, detection, evaluation, and treatment of high blood pressure. Hypertension. 2003;42(6): 1206-52.

23. Hankinson JL, Odencrantz JR, Fedan KB. Spirometric reference values from a sample of the general U.S. population. Am J Respir Crit Care Med. 1999;159(1):179-87.

24. Pulsford RM, Stamatakis E, Britton AR, Brunner EJ, Hillsdon MM. Sitting behavior and obesity: Evidence from the Whitehall II study. Am J Prev Med. 2013;44(2):132-8.

25. Borodulin K, Mäkinen TE, Leino-Arjas P, Tammelin $\mathrm{TH}$, Heliövaara M, Martelin T, et al. Leisure time physical activity in a 22-year follow-up among Finnish adults. Int J Behav Nutr Phys Act. 2012;9(1):121-6.

26. Golubic R, Ekelund U, Wijndaele K, Luben R, Khaw K-T, Wareham NJ, et al. Rate of weight gain predicts change in physical activity levels: A longitudinal analysis of the EPIC-Norfolk cohort. Int J Obes. 2013;37(3):404-9.

27. Lakerveld J, Dunstan D, Bot S, Salmon J, Dekker J, Niipels G, et al. Abdominal obesity, TV-viewing time and prospective declines in physical activity. Prev Med. 201 1;53(4-5):299-302.

28. Petersen L, Schnohr P, Sørensen TIA. Longitudinal study of the long-term relation between physical activity and obesity in adults. Int J Obes Relat Metab Disord. 2004;28(1):105-12.

29. Tremblay MS, Colley RC, Saunders TJ, Healy GN, Owen N. Physiological and health implications of a sedentary lifestyle. Appl Physiol Nutr Metab. 2010;35(6):725-40.

30. Owen N, Healy GN, Matthews CE, Dunstan DW. Too much sitting: The population health science of sedentary behavior. Exerc Sport Sci Rev. 2010;38(3):105-13.

31. Hamilton MT, Healy GN, Dunstan DW, Zderic TW, Owen N. Too little exercise and too much sitting: Inactivity physiology and the need for new recommendations on sedentary behavior. Curr Cardiovasc Risk Rep. 2008;2(4):292-8.
32. Owen N. Sedentary behavior: Understanding and influencing adults' prolonged sitting time. Prev Med. 2012;55(6):535-9. 
Chapt

照 


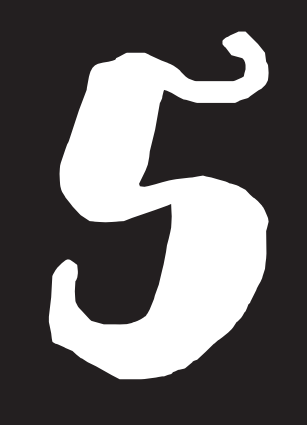

'Active sedentariness' is associated with cardio-metabolic outcomes and the metabolic syndrome: The AGES-Reykjavik Study

JULIANNE D. VAN DER BERG | HANS BOSMA | PAOLO CASEROTTI | GUDNY EIRIKSDOTTIR | NANNA Y. ARNARDOTTIR | DANE R. VAN DOMELEN | ROBERT J. BRYCHTA | KONG Y. CHEN | THORARINN SVEINSSON | ERLINGUR JOHANNSSON | LENORE J. LAUNER | VILMUNDUR GUDNASON | PALMI V. JONSSON | COEN D.A. STEHOUWER | TAMARA B. HARRIS | ANNEMARIE KOSTER 


\section{ABSTRACT}

Introduction: Sedentary behaviour can vary from sitting completely still to being more active while being sedentary (e.g. desk work ('active sedentariness')). Data on the health effects of active sedentariness are scarce but relevant; therefore we examined associations between objectively measured active sedentariness and cardio-metabolic outcomes and the metabolic syndrome.

Methods: 621 participants (73-98 years) of the AGES-Reykjavik Study wore in 2009-2010 a triaxial accelerometer. Active sedentariness was defined as sedentary time (0-99 counts per minute (cpm) in the vertical axis) with activity in the anteroposterior and/or mediolateral axes with an intensity $\geq 100 \mathrm{cpm}$. Outcome measures were: body mass index (BMI), waist circumference (WC), HDL cholesterol, triglycerides, fasting glucose, C-reactive protein, blood pressure and the metabolic syndrome.

Results: Compared to participants with the fewest active-sedentary minutes, those with more active-sedentary minutes had a lower BMI $\left(\mathrm{B}_{\text {quartile2 }}=-1.39 ; 95 \% \mathrm{Cl}=-2.3 ;-0.5\right) ; \mathrm{B}_{\text {quartile3 }}=-1.87 ;(-2.8 ;-0.9)$; $\left.\mathrm{B}_{\text {quartile4 }}=-3.38 ;(-4.3 ;-2.4)\right)$, a smaller WC $\left(\mathrm{B}_{\text {quartile2 }}=-2.95 ;(-5.4 ;\right.$ $\left.-0.5) ; \mathrm{B}_{\text {quartile } 3}=-3.47 ;(-6.0 ;-0.9) ; \mathrm{B}_{\text {quarile } 4}=-8.21 ;(-10.7 ;-5.7)\right)$, and a lower odds for the metabolic syndrome $\left(O R_{\text {quartile } 3}=0.58\right.$ $\left.(0.36 ; 0.95) ; \mathrm{OR}_{\text {quartile }}=0.36(0.22 ; 0.59)\right)$, even after adjustment for sedentary time and moderate to vigorous physical activity.

\section{Conclusions: Larger amounts of active sedentariness were} associated with improved metabolic outcomes, including a lower BMI, a smaller WC, and a lower odds for the metabolic syndrome. These findings suggest that activity accumulated during sedentary time could be beneficial to health. 


\section{INTRODUCTION}

Sedentary behaviour, such as sitting, watching TV or using the computer ${ }^{1}$ has been shown to be a risk factor for poor health. ${ }^{2-10}$ However, sedentary behaviour is not a single entity as it can vary from sitting completely still to being more active while being sedentary, for example when fidgeting or doing desk work ('active sedentariness'). Data on the health effects of this movement are scarce, because active sedentariness was previously disregarded or unmeasured.

To date, most accelerometry studies have used data that were recorded in the vertical axis only (i.e. the up-and-down direction) and data of movement in other directions, i.e. back-to-front and side-to-side, were not taken into account. Active sedentariness which is characterized by movement in the back-to-front and side-to-side directions, could therefore not be assessed. Since triaxial accelerometers record movement in all three directions, active sedentariness can be assessed using such a device. ${ }^{11}$ It is then possible to distinguish individuals with more movement during sedentary time from those with less movement during sedentary time.

We hypothesized that larger amounts of active sedentariness are associated with beneficial health outcomes. Examining whether active sedentariness could be relevant to health is essential to further explore and establish the associations of sedentary behaviour with health. Furthermore, it is important for the development of physical activity and sedentary behaviour guidelines, as even limited activity may influence physiologic mechanisms. Therefore, we examined whether active sedentariness is associated with cardio-metabolic outcomes and the metabolic syndrome independent of total sedentary time and moderate to vigorous physical activity (MVPA). We used triaxial accelerometers in a large sample of older adults who participated in the Age, Gene/Environment Susceptibility (AGES)-Reykjavik Study.

\section{$\underline{\text { METHODS }}$}

\section{Study population}

This study used a subsample of the Age, Gene/Environment Susceptibility (AGES)-Reykjavik Study cohort. The AGES-Reykjavik Study originates from the Reykjavik Study, which was started in 1967 and comprised a random sample of 30,795 participants born in 1907-1935 
and residing in Reykjavik, Iceland. The measurements for the AGES-Reykjavik Study took place from 2002 until 2006 among 5,764 participants of the original cohort. The follow-up measurements for the AGESII-Reykjavik Study took place in 2007-2011 among 3,41 1 participants. A full description of the AGES-Reykjavik Study can be found elsewhere. ${ }^{12}$

From April 2009 to June 2010, we studied a subcohort of 658 participants, ${ }^{13}$ who did not have severe cognitive dysfunction (Mini Mental State Examination $\geq 20$ ), and who were asked to wear an accelerometer for seven consecutive days. After excluding participants who had failed to record at least one valid day (at least $10 \mathrm{~h}$ of monitoring) ( $n=15)$ or who had missing data $(n=22)$, a total of 621 participants (239 men (38\%); aged 73 to 98 years) were included in the current analyses.

All participants gave written informed consent. The AGES-Reykjavik Study was approved by the institutional review boards of the National Institute on Aging, the National Bioethics Committee (VSN: 00-063) of Iceland and the Data Protection Authority of Iceland.

\section{Measurements}

Cardio-metabolic outcomes

Body mass index (BMI) was calculated using height and weight data $\left(\mathrm{kg} / \mathrm{m}^{2}\right)$ which were collected using standardized techniques. Waist circumference was measured twice using a tape that measures around the body covering the navel, while participants were standing. HDL cholesterol, triglycerides, plasma glucose levels, and C-reactive protein were measured in fasting blood samples and analysed on a Hitachi 912, using reagents from Roche Diagnostics. Triglycerides, fasting glucose and C-reactive were transformed using natural logarithm, due to a skewed distribution. Systolic and diastolic blood pressure were measured twice using a mercury sphygmomanometer. The metabolic syndrome was defined according to the ATPIII guidelines by the presence of 3 or more of the following criteria: (1) waist circumference $\geq 102 \mathrm{~cm}$ for men and $\geq 88 \mathrm{~cm}$ for women; (2) serum triglyceride level $\geq 1.7 \mathrm{mmol} / \mathrm{L}$; (3) HDL cholesterol level $<1.03 \mathrm{mmol} / \mathrm{L}$ for men and $<1.3 \mathrm{mmol} / \mathrm{L}$ for women; (4) fasting glucose level $\geq 5.6 \mathrm{mmol} / \mathrm{L}$ or use of antidiabetic medications (insulin or oral agents); or (5) systolic blood pressure $\geq 130 \mathrm{mmHg}$ and/or diastolic blood pressure $\geq 85 \mathrm{mmHg}$, and/or use of antihypertensive medications. ${ }^{14}$

\section{Sedentary behaviour variables}

Sedentary time was recorded using an accelerometer (ActiGraph GT3X, Ft. Walton Beach, FL, USA), which was attached to a belt and was worn on the right hip. ${ }^{13}$ Participants were 
instructed to wear the accelerometer for seven consecutive days and only remove the monitor before going to bed and during showering, bathing, swimming and other water-related activities.

The triaxial ActiGraph recorded activity on the vertical, anteroposterior (back-to-front) $(A P)$, and mediolateral (side-to-side) (ML) axes. Raw accelerometry data were processed and averaged per minute using customized software written in MATLAB R2006a (The MathWorks, Natick MA, USA). Non-wear time was defined as any interval $\geq 60$ consecutive min of zero counts in all three axes, allowing a period of up to 2 min of nonzero counts $<100$ in the vertical axis. A valid day was defined as at least $10 \mathrm{~h}$ of monitoring. Sedentary time was defined as 0-99 counts per minute (cpm) in the vertical axis. Active sedentariness was defined sedentary time (0-99 cpm in the vertical axis) with activity in the AP and/or ML axes with an intensity $\geq 100 \mathrm{cpm}$. The measure used in our analyses was the number of active-sedentary minutes per day. A sedentary break was defines as each minute in which $\geq 100$ counts were recorded in the vertical axis that was preceded by at least 1 min of $<100$ counts.

\section{Covariates}

Covariates were extracted from questionnaires and included sex, age, level of education (assessed in the AGES-Reykjavik Study), smoking status (measured in the AGES-Reykjavik Study), alcohol consumption (assessed in the AGES-Reykjavik Study), health status, mobility limitation, and medication use. Level of education was categorized into college/university, secondary, and primary, and smoking status into never smoker, previous smoker, and current smoker. The intake of alcohol in grams/week was used for the alcohol consumption covariate. Health status was defined as having one or more of the following conditions: heart disease, transient ischemic attack (TIA), stroke, or type 1 or type 2 diabetes mellitus. Mobility limitation was defined as having difficulty walking 500 m or climbing 10 steps without resting. Medication use was dichotomized (yes/no) for the use of antidiabetic and antihypertensive medication. Daily minutes of sedentary time and daily minutes of MVPA (MVPA $\geq 2020 \mathrm{cpm}$ ) were derived from the accelerometry data. BMI was calculated using height and weight measures $\left(\mathrm{kg} / \mathrm{m}^{2}\right)$.

\section{Statistical analysis}

For descriptive purposes, the exposure variable, that is, the active-sedentary minutes, was split into sex-specific quartiles because the associations were not linear and to obtain equal 
distributions of men and women (60 men and 95 women in each quartile). The ranges of the quartiles of all participants were: 48.0-1 12.7, 103.4-145.3, 129.4-177.4, and 161.3-330.9 $\mathrm{min} /$ day. The ranges of the quartiles for men were: 54.5-103.1, 103.4-129.3, 129.4-161.1, 161.-330.9 min/day, and 48.0-112.7, 113.0-145.3, 145.4-177.4, 177.7-306.2 min/day for women. Descriptive characteristics and sedentary behaviour characteristics were summarized as mean with standard deviations (SD) or as number and percentages. Non-normally distributed variables were described using the median and the interquartile range. Chi-square tests, ANOVA and Kruskal-Wallis tests were conducted as appropriate to examine differences between the sex-specific quartiles of active-sedentary minutes. Linear regression analysis was used to examine whether active sedentariness was associated with the cardio-metabolic outcomes (analyses were run separately for each outcome). The associations of all models were adjusted for daily minutes of sedentary time (model 1). In models 2 the associations were additionally adjusted for sex, age, level of education, and daily minutes of MVPA. In models 3 the associations were additionally adjusted for smoking status, alcohol consumption, health status (the metabolic syndrome was not adjusted for diabetes mellitus), and mobility limitation. In models 2 the associations with HDL cholesterol, triglycerides, and C-reactive protein were also adjusted for $\mathrm{BMl}$; the association with fasting glucose was also adjusted for BMI and antidiabetic medication; and the associations of blood pressure were also adjusted for $\mathrm{BMI}$ and antihypertensive medication. Results are reported as unstandardized coefficients (B), back-transformed from the natural log scale for the non-normally distributed outcomes, and odds ratios (OR) for the metabolic syndrome. Interactions of the quartiles of active-sedentary minutes with sex were not statistically significant $(P>0.10)$, and therefore the analyses were conducted for men and women together. All analyses were conducted with IBM SPSS Statistics 20.0 (IBM Corp. Armonk, NY, USA). Significance level was set at $<0.05$.

\section{RESULTS}

The overall study population had an average age of $80.0 \pm 4.7$ years, and $38 \%$ of them were men. Almost $50 \%$ of this population (49.9\%) provided 7 valid days of accelerometry data (>10 h of monitoring), with $27.9 \%$ providing 6 days, $10.0 \% 5$ days, $4.0 \% 4$ days and $4.3 \% 3$ days. Descriptive characteristics of the study population according to the 
quartiles of active sedentariness are presented in Table 5.1. Participants with more active-sedentary minutes were less likely to be obese and less often had limited mobility. They also had a lower BMI, a smaller waist circumference, a higher HDL cholesterol level, and lower levels of triglycerides, fasting glucose, and C-reactive protein, and were less often likely to have the metabolic syndrome (all $P<0.05$ ). Systolic and diastolic blood pressure were not statistically significantly different between the quartiles. The amount of time spend sedentary was larger among participants with more active-sedentary minutes $(P=0.012)$; however, the percentage time spent sedentary did not differ between the quartiles $(P=0.463)$. The percentage active-sedentary minutes of total sedentary time was significantly higher among those with more active-sedentary minutes $(P<0.001)$.

Figure 5.1 shows the distribution of the number of metabolic syndrome criteria in the quartiles. Three or more metabolic syndrome criteria were present in $51 \%$ of the participants with the fewest number of active-sedentary minutes (quartile 1). This percentage was lower for those with more active-sedentary minutes; $43.5 \%$ (quartile 2), 38.8\% (quartile 3 ), and $27.8 \%$ (quartile 4). Five metabolic syndrome criteria were present in $8.3 \%$ of the participants in quartile 1 , and in $5.2 \%, 3.9 \%$, and $1.9 \%$ of the participants in quartiles 2 , 3 , and 4 , respectively.

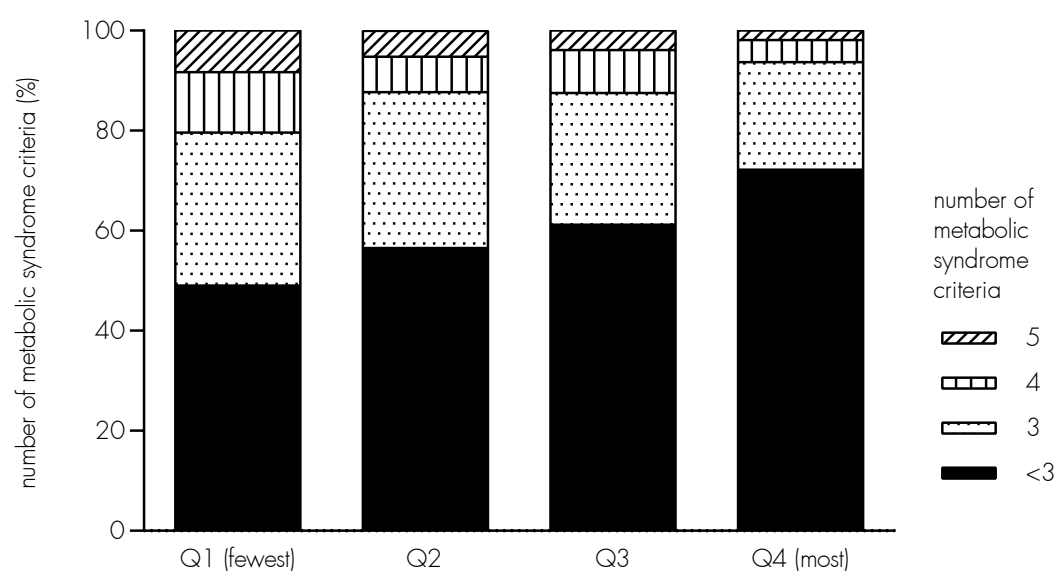

Quartiles of active-sedentary minutes

Figure 5.1: Distribution of the number of metabolic syndrome criteria in the sex-specific quartiles of active-sedentary minutes. Ranges of the the quartiles were quartile 1: 48.0-1 12.7; quartile 2: 103.4-145.3; quartile 3: 129.4-177.4; quartile 4: 161.3-330.9. $n=621 ; 60$ men and 95 women in each quartile. 


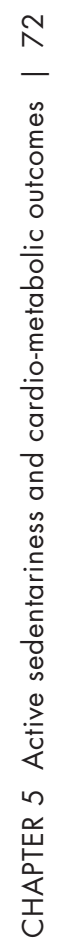

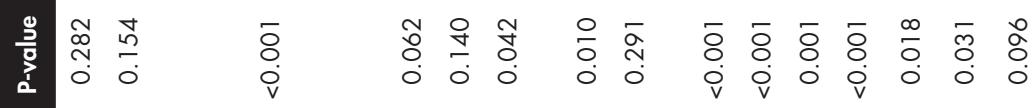

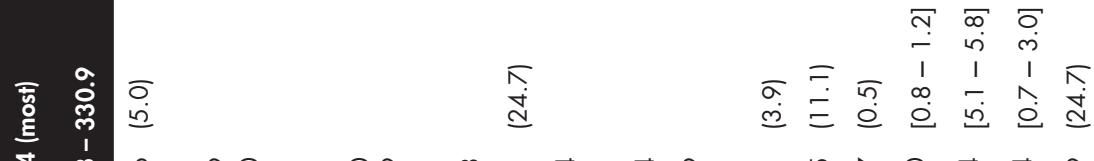

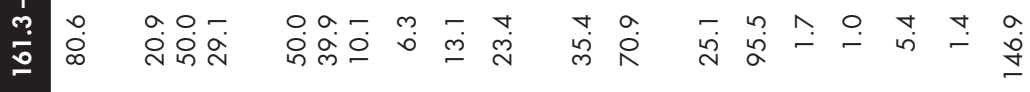

ָุ

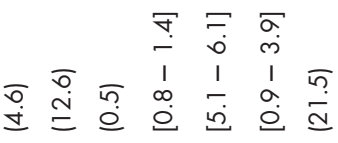

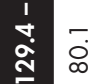

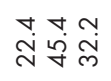

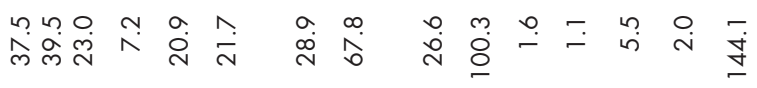

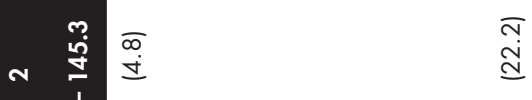<smiles>C1=NCCCC1</smiles>

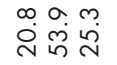

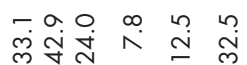
ڤેํำ

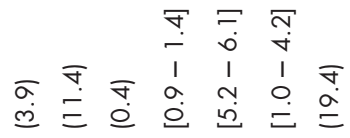
突

$\stackrel{\text { m }}{\stackrel{\infty}{\infty}}$ ヘิ 


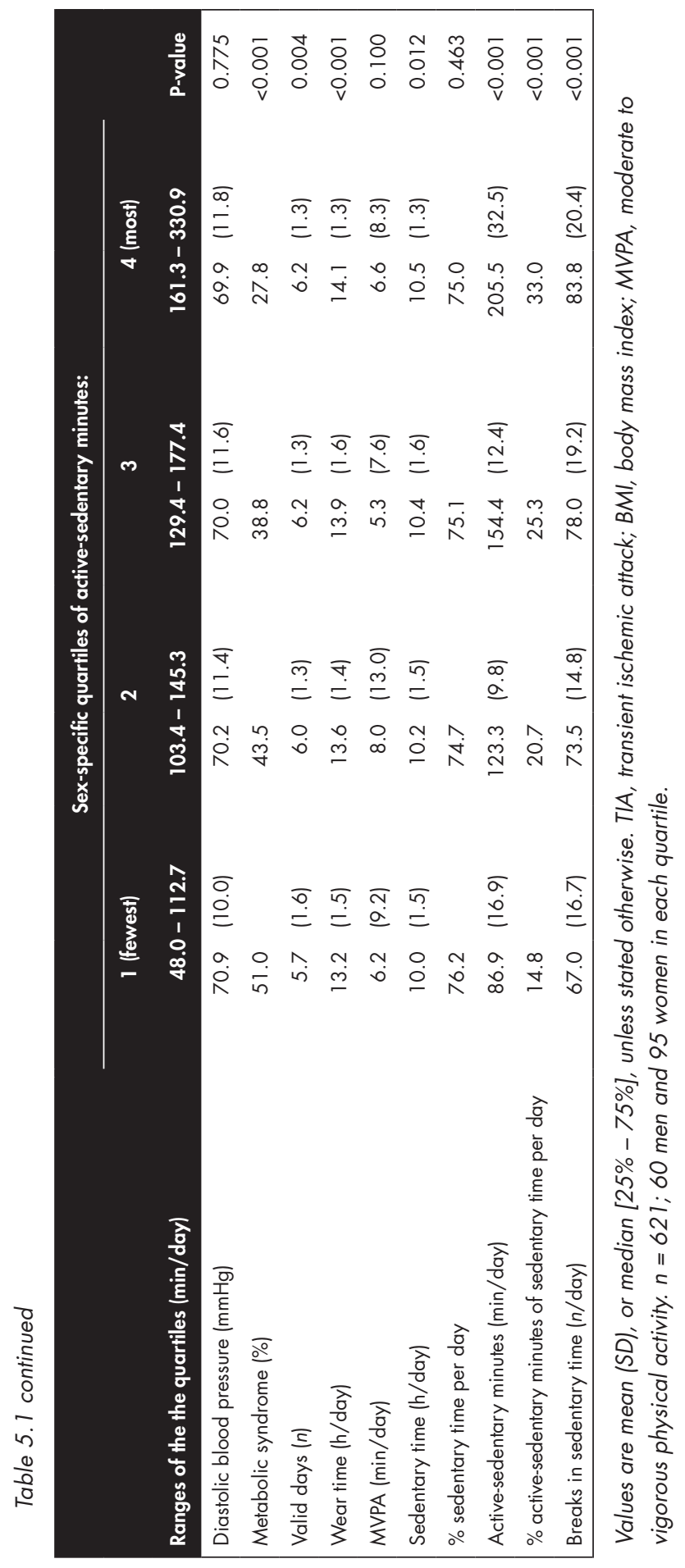




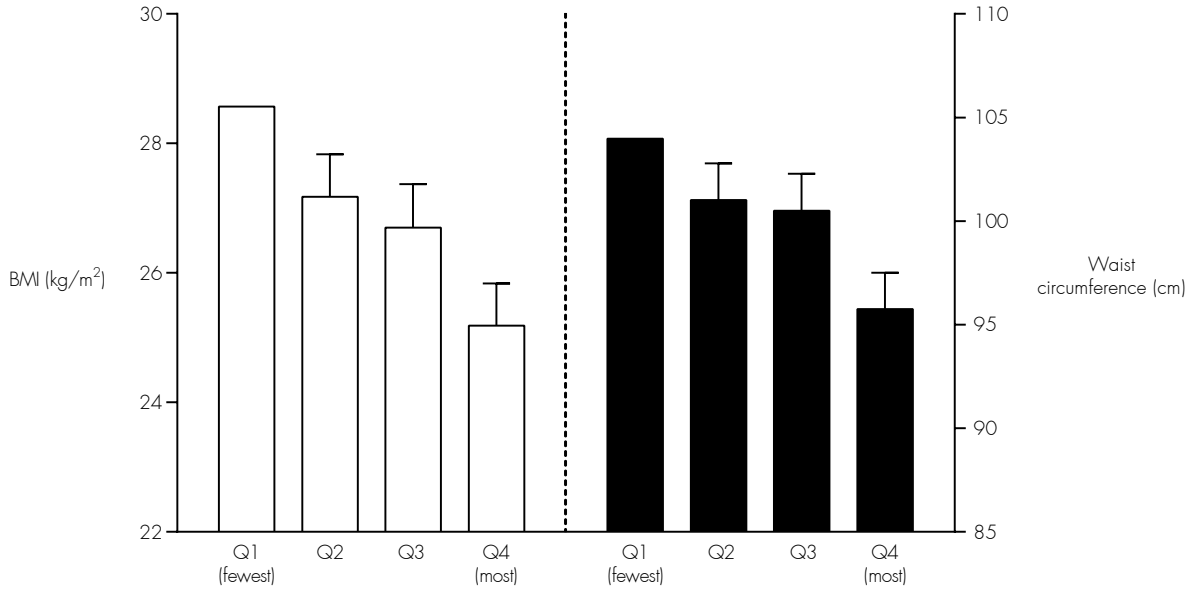

Quartiles of active-sedentary minutes

Figure 5.2: Sex-specific quartiles of active-sedentary minutes and body mass index (BMI) and waist circumference. Data are mean values with $95 \%$ confidence intervals, adjusted for sex, age, level of education, daily minutes of sedentary time, daily minutes of MVPA, smoking status, alcohol consumption, health status, and mobility limitation. Ranges of the quartiles were quartile 1: 48.0-1 12.7; quartile 2: 103.4-145.3; quartile 3: 129.4-177.4; quartile 4: 161.3-330.9. $n=621 ; 60$ men and 95 women in each quartile.

Figure 5.2 shows that compared to those with the fewest number of active-sedentary minutes (quartile 1), participants with more active-sedentary minutes (quartiles 2, 3, 4) had a significantly lower $\mathrm{BMI}\left(\mathrm{B}_{\text {quartile2 }}=-1.39 ;(95 \% \mathrm{Cl}=-2.33 ;-0.46) ; \mathrm{B}_{\text {quartile3 }}=-1.87 ;(-2.82\right.$; $-0.92) ; B_{\text {quartile4 }}=-3.38(-4.32 ;-2.45)$; Table 5.2) and a smaller waist circumference $\left(B_{\text {quartile2 }}=-2.95(-5.44 ;-0.46) ; B_{\text {quartile3 }}=-3.47 ;(-6.01 ;-0.93) ; B_{\text {quartile4 }}=-8.21 ;(-10.72 ;-5.71)\right)$, after adjustment for sedentary time, MVPA, demographic factors (sex, age, educational level) and health factors (smoking status, alcohol consumption, health status, and mobility limitation). The number of active-sedentary minutes was also statistically significantly associated with the cardio-metabolic outcomes HDL cholesterol, triglycerides, fasting glucose, C-reactive, systolic blood pressure, and the metabolic syndrome, but not with diastolic blood pressure, after adjusting for total sedentary time, MVPA, and demographic factors (model 2; Table 5.2). After additional adjustment for health factors, BMI, and medication use (model 3), the results show that more active-sedentary minutes remained statistically significantly associated with a higher $\mathrm{HDL}$ cholesterol level $\left(\mathrm{B}_{\text {quartile4 }}=0.13 ;(0.04 ; 0.22)\right)$, a lower triglycerides level $\left(B_{\text {quartile }}=0.91 ;(0.84 ; 0.99)\right.$; back-transformed from log scale), and a 
higher systolic blood pressure $\left(B_{\text {quartile4 }}=5.10 ;(0.12 ; 10.07)\right)$, but not with fasting glucose and C-reactive protein. The differences between models 2 and 3 were predominantly caused by BMI. Having more active-sedentary minutes remained also statistically significantly associated with a lower odds for the metabolic syndrome in model $3\left(O R_{\text {quartile } 3}=0.58\right.$ (0.36;0.95); $\left.O R_{\text {quartile4 }}=0.36(0.22 ; 0.59)\right)$.

When active sedentariness was expressed in hours and was analysed as a continuous variable, statistically significant associations were found for BMI, waist circumference, levels of HDL cholesterol and triglycerides, and the metabolic syndrome in model 2 (Table 5.2). Each hour of activity during sedentary time was estimated to result in a lower $\mathrm{BMI}(\mathrm{B}=-1.73 ;(-2.15 ;-1.31))$, a smaller waist circumference $(\mathrm{B}=-3.85 ;(-4.98 ;-2.72))$, a higher $\mathrm{HDL}$ cholesterol level $(B=0.06 ;(0.01 ; 0.10))$, a lower triglycerides level $(B=0.96$; $(0.95 ; 0.99)$ back-transformed from log scale), and a lower odds for the metabolic syndrome $(O R=0.59 ;(0.47 ; 0.75))$.

In additional analyses the associations between active-sedentary minutes and all outcomes were further adjusted for wear time and also for the number of sedentary breaks. This resulted in similar associations (data not shown). 


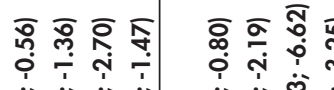

অ 

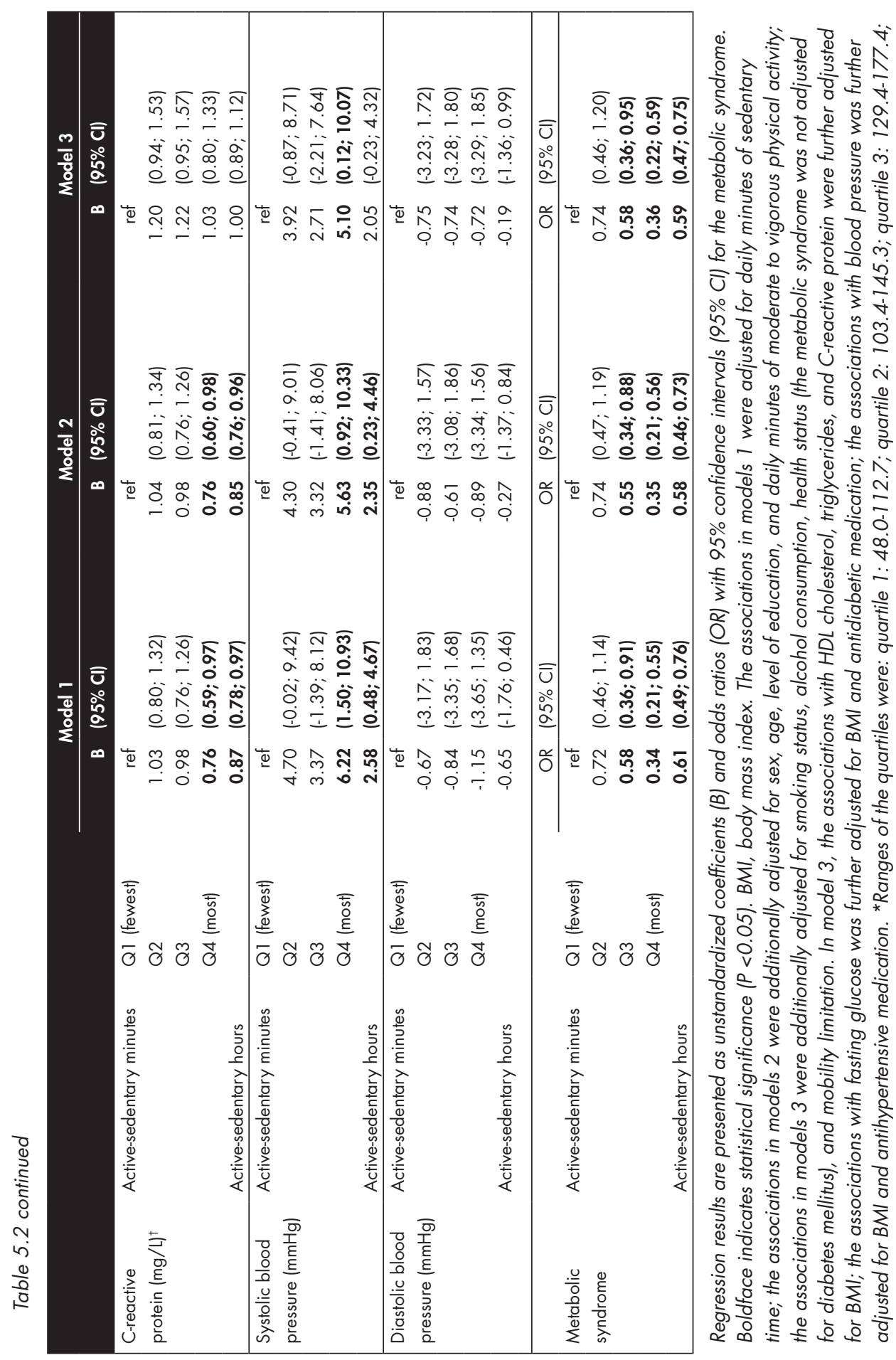

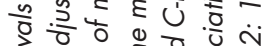

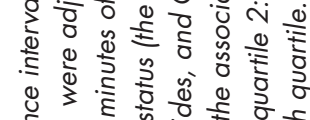

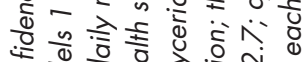
$\therefore$ 过

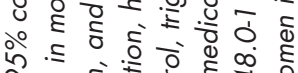
人

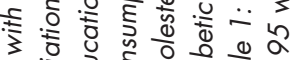

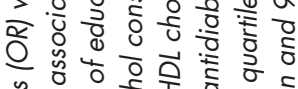

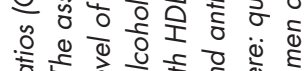

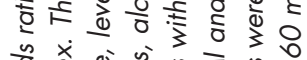

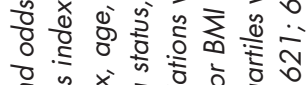
万人

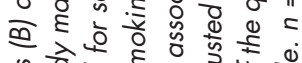

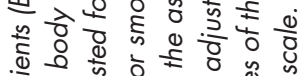

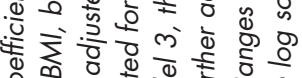

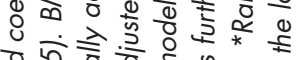

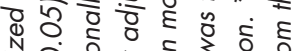

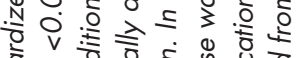
oㄴ

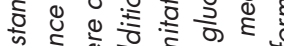
ป ठ के

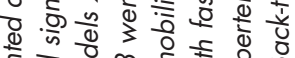
के के

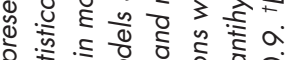
है.

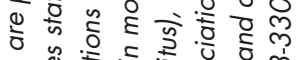

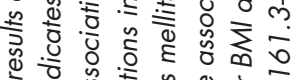
ธิ

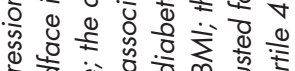

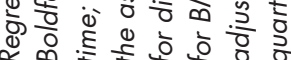




\section{DISCUSSION}

To our knowledge, this is the first study that has examined the potential health effects of activity accumulated during sedentary time using triaxial accelerometry data. The results show that larger amounts of active sedentariness were statistically significantly associated with beneficial metabolic outcomes, even after adjustment for relevant covariates including sedentary time and MVPA. Compared to those who had the fewest number of activesedentary minutes, participants with the most active-sedentary minutes had an $3.4 \mathrm{~kg} / \mathrm{m}^{2}$ lower BMl and an $8.2 \mathrm{~cm}$ smaller waist circumference. Those with the most active-sedentary minutes had a $0.13 \mathrm{mmol} / \mathrm{L}$ higher $\mathrm{HDL}$ cholesterol level and a 0.91 times lower triglycerides level, but also a $5.10 \mathrm{mmHg}$ higher systolic blood pressure than those who had the fewest number of active-sedentary minutes. In addition, those with the most active-sedentary minutes had a 0.36 times lower odds for the metabolic syndrome. No statistically significant associations were seen for levels of fasting glucose and C-reactive protein, and diastolic blood pressure in the fully adjusted model. Given the large amounts of time that people spend being sedentary, ${ }^{13,15,16}$ the finding that even limited activity accumulated in a sedentary position is associated with beneficial metabolic outcomes is important.

To date, no other researchers have examined objectively measured activity accumulated during sedentary time, with the exception of Bankoski and colleagues. ${ }^{6}$ They examined the degree of activity during sedentary time and found that a higher degree of activity was associated with a decreased likelihood of metabolic syndrome. This was a first indication that activity accumulated during sedentary time could be relevant. The results of the current study are in line with their findings. Of note, in Bankoski's study activity accumulated during sedentary time was based on data of the vertical axis only, while we used triaxial data. In addition, a recently published study using self-reporting measures showed that limited movement involved in fidgeting may protect against adverse health effects of sitting. ${ }^{17}$ Although that study used subjective measures, their results, those of Bankoski, and ours suggest the potential importance of limited movement. This may be of particular importance for those who are unable to meet physical activity guidelines. Future studies are therefore encouraged to measure this type of activity and further examine its impact on health.

In the current study, more active-sedentary minutes were associated with a considerably lower BMI, smaller waist circumference and lower odds ratio for the metabolic syndrome, which suggests that active sedentariness may be a relevant aspect of sedentary behaviour. 
Although statistically significant for levels of HDL cholesterol and triglycerides, the results for these metabolic outcomes were small and therefore may be not clinically relevant. However, due to additional adjustment for BMI, these results may have been to some extent subject to overadjustment, as BMI may be part of the pathway between active sedentariness and metabolic outcomes. The interpretation of the blood pressure findings is difficult, as healthy blood pressure levels defined for young and middle-aged adults (systolic blood pressure $<130 \mathrm{mmHg}$ and/or diastolic blood pressure $<85 \mathrm{mmHg}$ ) may not apply for old or very old adults. ${ }^{18}$ The physiological mechanisms underlying the combined effects of being in a sedentary position and activity while being sedentary have not yet been studied. It is therefore difficult to clarify the findings of the present study. The mechanisms may be similar to those demonstrated in studies of non-exercise activity, which have shown that limited movement (fidgeting-like activities) was associated with substantial increases in energy expenditure and was inversely related to body weight and body weight gain. ${ }^{19-21}$ Further examination of the underlying mechanisms requires physiological studies.

A major strength of the present study is its unique examination of activity accumulated during sedentary time using triaxial accelerometry data. Although most currently available accelerometers provide data from three axes, such data have rarely been used. These data can, however, contribute to a more comprehensive examination of sedentary behaviour, which is essential to further establish the associations of sedentary behaviour with health. Another strength of this study was that almost all participants (92\%) provided at least four valid days of accelerometry data, and the recorded sedentary time was comparable with the amounts described in other studies among older adults..$^{3,16,22,23}$ In addition, the adjustment for sedentary time and MVPA, as well as several demographic and health factors, exclude the possibility that these covariates account for the effects of activity accumulated during sedentary time on the cardio-metabolic outcomes.

This study also has limitations which should be considered. An important limitation is the uncertainty regarding the sedentary position in which the activity was recorded. A hip-worn accelerometer may not accurately distinguish between sitting and standing, thus activity in the AP and ML axes may have been recorded during standing as well as during sitting. So, future studies may include posture-based data to obtain more accurate measures. Also, it could be possible that the chosen cut-off point for sedentary time 10-99 $\mathrm{cpm}$ ) may not be an appropriate reflection of sedentary time, although this cut-off point has been evaluated for validity. ${ }^{3}$ In addition, it is not clear what type of movement is involved when activity in the AP and/or ML axes is examined. Such movements could, 
for instance, include those involved in transitions from sitting to standing or vice versa. However, these movements did not account for the effects found in this study, as the results did not change after additionally adjusting for sedentary breaks. Further, it is not clear what the intensity of the activity accumulated during sedentary time was. Only one experimental study has demonstrated that during sedentary activities such as playing cards, significant amounts of activity counts in the AP and/or ML axes were recorded using a tri-axial hip-worn accelerometer. ${ }^{11}$ There is therefore a need for validation studies to examine the type and intensity of movement that account for this activity accumulated during sedentary time. Other limitations of the current study were its cross-sectional design, which limits the possibility of identifying causal relationships, and residual confounding due to unmeasured, but possibly relevant factors such as diet. Finally, the study population consisted of a relatively healthy subsample of older adults, which could limit the generalization of our results.

To conclude, this is the first study that used triaxial accelerometry data to examine associations between active sedentariness and cardio-metabolic outcomes and the metabolic syndrome. More activity in the AP and/or ML axes accumulated during sedentary time was associated with a considerably lower BMI and smaller waist circumference, as well as lower HDL cholesterol and higher triglycerides levels. In addition, having more active-sedentary minutes was associated with a lower odds for the metabolic syndrome. These findings suggest that active sedentariness may be a relevant aspect of sedentary behaviour. Validation studies are needed to examine the type and intensity of movement involved in activity accumulated during sedentary time. Future sedentary behaviour studies are encouraged to including triaxial accelerometry data to determine whether the results of the current study can be reproduced. 


\section{REFERENCES}

1. Atkin AV, Gorely T, Clemes SA, Yates T, Edwardson $C$, Brage $S$, et al. Methods of measurement in epidemiology: Sedentary behaviour. Int J Epidemiol. 2012;41(5): 1460-71.

2. Koster A, Caserotti P, Patel KV, Matthews CE, Berrigan D, Van Domelen DR, et al. Association of Sedentary Time with Mortality Independent of Moderate to Vigorous Physical Activity. PLoS One. 2012;7(6):e37696.

3. Matthews C, George S, Moore S, Bowles H, Blair A, Park Y, et al. Amount of time spent in sedentary behaviors and cause-specific mortality in US adults. Am J Clin Nutr. 2012;95:437-45.

4. Grøntved A. Television viewing and risk of type 2 diabetes, cardiovascular disease, and all-cause mortality. JAMA. $2011 ; 305(23)$ :2448-55.

5. Chau JY, Grunseit AC, Chey T, Stamatakis E, Brown WJ, Matthews CE, et al. Daily sitting time and all-cause mortality: A meta-analysis. PLoS One. 2013;8(1 1):e80000.

6. Bankoski A, Harris TB, McClain JJ, Brychta RJ, Caserotti $P, C$ hen KY, et al. Sedentary activity associated with metabolic syndrome independent of physical activity. Diabetes Care. $2011 ; 34(2): 497-503$.

7. Edwardson CL, Gorely T, Davies M, Gray L, Khunti K, Wilmot EG, et al. Association of sedentary behaviour with metabolic syndrome: A meta-analysis. PLoS One. 2012;7(4):e34916.

8. Healy GN, Dunstan DW, Salmon J, Shaw JE, Zimmet PZ, Owen N. Television time and continuous metabolic risk in physically active adults. Med Sci Sports Exerc. 2008;40(4):639-45.

9. Wilmot EG, Edwardson CL, Achana FA, Davies M, Gorely T, Gray L, et al. Sedentary time in adults and the association with diabetes, cardiovascular disease and death: Systematic review and meta-analysis. Diabetologia. 2012;55(11):2895-905.

10. Ford ES, Caspersen CJ. Sedentary behaviour and cardiovascular disease: A review of prospective studies. Int J Epidemiol. 2012;41(5):1338-53.

11. Van der Berg JD, Stehouwer CDA, Bosma H, Koster A. Measuring back-to-front and side-toside movement during sedentary activities using a hip-worn tri-axial accelerometer. J Sports Sci. 2016;2:1-7.
12. Harris $T B$, Launer $L$, Eiriksdottir $G$, Kjartansson O, Jonsson P, Sigurdsson G, et al. Age, Gene/ Environment Susceptibility-Reykjavik Study: Multidisciplinary applied phenomics. Am J Epidemiol. 2007;165(9): 1076-87.

13. Arnardottir NY, Koster A, Van Domelen DR, Brychta RJ, Caserotti P, Eiriksdottir G, et al. Objective measurements of daily physical activity patterns and sedentary behaviour in older adults: Age, Gene/Environment Susceptibility-Reykjavik Study. Age Ageing. 2012;0:1-7.

14. Grundy SM, Cleeman JI, Daniels SR, Donato KA, Eckel RH, Franklin B a, et al. Diagnosis and management of the metabolic syndrome: An American Heart Association/National Heart, Lung, and Blood Institute Scientific Statement. Circulation. 2005; 112(17):2735-52.

15. Matthews CE, Chen KY, Freedson PS, Buchowski MS, Beech M, Pate RR, et al. Amount of time spent in sedentary behaviors in the United States, 20032004. Am J Epidemiol. 2008;167(7):875-81.

16. Davis MG, Fox KR, Hillsdon M, Sharp DJ, Coulson JC, Thompson JL. Objectively measured physical activity in a diverse sample of older urban UK adults. Med Sci Sports Exerc. 2011 ;43(4):647-54.

17. Hagger-Johnson G, Gow A, Burley V, Greenwood D, Cade JE. Sitting time, fidgeting, and allcause mortality in the UK Women's Cohort Study. Am J Prev Med. 2016;50(2):154-60.

18. Muller M, Smulders YM, de Leeuw PW, Stehouwer CDA. Treatment of hypertension in the oldest old: A critical role for frailty? Hypertension. 2014;63(3):433-41.

19. Johanssen D, Ravussin E. Spontaneaous physical activity: Relationship between figeting and body weight control. Curr Opin Endocrinol Diabetes Obes. 2008;15(5):409-15.

20. Levine JA. Nonexercise activity thermogenesis (NEAT): Environment and biology. Am J Physiol Endocrinol Metab. 2004;286(5):E675-85

21. Levine JA, Schleusner SJ, Jensen MD. Energy expenditure of nonexercise activity. Am J Clin Nutr. 2000;72(6):1451-4.

22. Evenson K, Buchner D, Morland K. Objective measurement of physical activity and sedentary behavior among US adults aged 60 years or older. Prev Chronic Dis. 2011;9(2):110109.

23. Shiroma EJ, Freedson PS, Trost SG, Lee I-M. Patterns of accelerometer-assessed sedentary behavior in older women. JAMA. 2013;310(23):2562. 
Chapt

照 


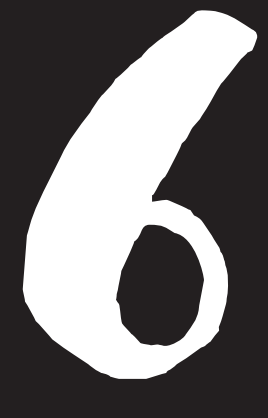

Theoretical effects of replacing sedentary time with standing or stepping on cardio-metabolic outcomes and type 2 diabetes: The Maastricht Study

JULIANNE D. VAN DER BERG | JEROEN H.P.M. VAN DER VELDE | ELLIS A.C. DE WAARD | HANS BOSMA | HANS H.C.M. SAVELBERG | NICOLAAS C. SCHAPER | JOOP P.W. VAN DEN BERGH | PIET P.M.M. GEUSENS | MIRANDA T. SCHRAM | SIMONE J.S. SEP | CARLA J.H. VAN DER KALLEN | RONALD M.A. HENRY | PIETER C. DAGNELIE | SIMONE J.P.M. EUSSEN | MARTIEN C.J.M. VAN DONGEN | SEBASTIAN KÖHLER | ABRAHAM A. KROON | COEN D.A. STEHOUWER | ANNEMARIE KOSTER 


\section{$\underline{\text { AB STRACT }}$}

Background: Sedentary time has been associated with detrimental health effects, so recommendations for reducing sedentary time have been developed. As reducing sedentary time inevitably results in more non-sedentary time, effects of this reduction may depend on the activity with which it is replaced. Therefore, we examined associations of theoretical reallocations of sedentary time to standing or stepping with cardio-metabolic outcomes and type 2 diabetes (T2DM).

Methods: We included 2,213 participants (51\% men, age (mean $\pm S D$ ): $60.0 \pm 8.1$ years) of The Maastricht Study who were asked to wear an activPAL3 accelerometer $24 \mathrm{~h} /$ day for 8 consecutive days. We calculated daily amounts of sedentary, standing, and stepping time. An isotemporal substitution modelling approach was applied to examine replacement effects on waist circumference (WC), body mass index (BMI), blood pressure, levels of cholesterol, triglycerides, glucose, and insulin, $\mathrm{HbAlc}$, the metabolic syndrome and T2DM.

Results: Replacement of sedentary time (30 min/day) with stepping was associated with a $1.42 \mathrm{~cm}(\mathrm{~B}=-1.42 ;(95 \% \mathrm{Cl}=-1.78$; -1.06)) smaller WC, a $0.48 \mathrm{~kg} / \mathrm{m}^{2}(B=-0.48 ;(-0.62 ;-0.35))$ lower $\mathrm{BMI}$, a lower odds for the metabolic syndrome $(\mathrm{OR}=0.71$; $(0.66 ; 0.78))$ and T2DM (OR $=0.79 ;(0.72 ; 0.87))$, and improved cholesterol, triglyceride, glucose and insulin levels. Replacing sedentary time with standing was associated with favourable outcomes in WC, and cholesterol, triglyceride and insulin levels, and a lower odds for the metabolic syndrome and T2DM.

Conclusions: Theoretical replacements of sedentary time with any type of non-sedentary time (standing/stepping) were associated with beneficial metabolic outcomes. These results could be important for the general population, including those who cannot meet the physical activity guidelines, and consideration should be given to developing recommendations for daily reallocating sedentary time. 


\section{INTRODUCTION}

Sedentary behaviour, which refers to any waking behaviour that is characterized by an energy expenditure $\leq 1.5$ metabolic equivalents (METs) while in a sitting or reclining posture such as watching TV or using the computer, ${ }^{1}$ has been associated with several adverse health outcomes, including cardio-metabolic risk factors (e.g. waist circumference, cholesterol and triglyceride levels, insulin resistance), 2,3 cardiovascular disease, ${ }^{4,5}$ type 2 diabetes, ${ }^{4,5}$ and premature mortality. ${ }^{4,5}$

Therefore, in some countries, health guidelines to reduce sedentary time have been developed by public health organisations and scientific institutions, ${ }^{6,7}$ and the Australian government has even published a sedentary behaviour guideline. ${ }^{8}$ However, specific recommendations for the amount of sedentary time that should be reduced are lacking. To develop these recommendations, studies on the health effects of reducing sedentary time are needed. More specifically, as reducing sedentary time during waking time inevitably results in larger amounts of non-sedentary time which can vary from light physical activity (LPA) to vigorous physical activity, the effects of reducing sedentary time may depend on the activity with which it is replaced. Therefore, studies examining the effects of reduced sedentary time replaced with other types of behaviours are required.

An isotemporal substitution model (ISM) can be used to examine the effects of the theoretical replacement of sedentary time by non-sedentary time like standing or stepping. ${ }^{9}$ Previous studies that have used an ISM approach demonstrated that a theoretical replacement of sedentary time with LPA or moderate to vigorous physical activity (MVPA) was associated with favourable health outcomes, including a reduction in body mass index ${ }^{10-13}$ and waist circumference, ${ }^{10,12-15}$ and improved markers of insulin sensitivity, ${ }^{14,16}$ levels of cholesterol, ${ }^{11,13-15}$ triglyceride ${ }^{11,13-15}$ and glucose. ${ }^{13,14,16}$

Although previous studies have used accelerometry data to assess sedentary time, in most studies sedentary time was solely based on acceleration data which cannot discriminate between postures. Consequently standing time may have been misclassified as sedentary time or vice versa. ${ }^{17,18}$ Therefore, we used the activPAL accelerometer which classifies sedentary behaviour (sitting or lying) using data on posture combined with acceleration, as this has shown to be an accurate method for assessing sedentary behaviour. ${ }^{18,19}$

The aim of this study was to examine the cross-sectional associations of a theoretical reallocation of sedentary behaviour (sitting or lying) to standing or stepping with 
cardio-metabolic outcomes, the metabolic syndrome and type 2 diabetes in a large sample of adults who participated in The Maastricht Study.

\section{METHODS}

\section{Study population}

We used data from The Maastricht Study, an observational prospective population-based cohort study. The rationale and methodology have been described previously. ${ }^{20}$ In brief, the study focuses on the aetiology, pathophysiology, complications and comorbidities of type 2 diabetes mellitus (T2DM) and is characterized by an extensive phenotyping approach. Eligible participants were individuals aged between 40 and 75 years and living in the southern part of the Netherlands. Participants were recruited through mass media campaigns and from the municipal registries and the regional Diabetes Patient Registry via mailings. Recruitment was stratified according to known T2DM status, with an oversampling of individuals with T2DM, for reasons of efficiency. This study included cross-sectional data from 3,451 participants, who completed the baseline survey between November 2010 and September 2013. Participants with type 1 diabetes, latent auto-immune diabetes in adults (LADA), steroid-induced diabetes or diabetes after pancreatectomy $(n=41)$ were excluded. After further successively excluding participants who did not receive an accelerometer due to logistics ( $n=668$ ), whose accelerometer measurement failed ( $n=135$ ) or who had other missing data $(n=394)$, a total of 2,213 participants were included in the present analyses.

The study was approved by the institutional medical ethical committee (NL3 1329.068.10) and the Minister of Health, Welfare and Sports of the Netherlands (Permit 131088-105234-PG). All participants gave written informed consent.

\section{Measurements}

\section{Cardio-metabolic outcomes}

The following cardio-metabolic outcomes were used: waist circumference, body mass index (BMI), office blood pressure, HDL cholesterol, total-to-HDL cholesterol ratio, triglycerides, fasting glucose, $2 \mathrm{~h}$ post-load glucose, $\mathrm{HbAlc}$, fasting insulin, the metabolic syndrome, and T2DM. 
Waist circumference was measured in duplicate, midway between the lower rib margin and the iliac crest at the end of expiration, both to the nearest $0.5 \mathrm{~cm}$. Weight and height were measured without shoes and wearing light clothing using a scale and stadiometer to the nearest $0.5 \mathrm{~kg}$ or $0.1 \mathrm{~cm}$ (Seca, Hamburg, Germany). BMI was calculated as $\mathrm{kg} / \mathrm{m}^{2}$. Office blood pressure was determined three times on the right arm after a 10-minute rest period, using a non-invasive blood pressure monitor (Omron 705IT, Japan). All available measurements were used to calculate the average blood pressure. Fasting blood samples were used for laboratory assessment of levels of total cholesterol, $\mathrm{HDL}$ cholesterol, triglycerides, glucose, $\mathrm{HbAlc}$, and insulin. ${ }^{20}$ Total-to-HDL cholesterol ratio was calculated by dividing total cholesterol by HDL cholesterol. To determine $2 \mathrm{~h}$ post-load glucose, all participants (except those who use insulin) underwent a standardized $75 \mathrm{~g}$ oral glucose tolerance test after an overnight fast as described elsewhere. ${ }^{20}$ The metabolic syndrome was defined according to the ATPIII guidelines by the presence of 3 or more of the following criteria: (1) waist circumference $\geq 102 \mathrm{~cm}$ for men and $\geq 88 \mathrm{~cm}$ for women; (2) serum triglyceride level $\geq 1.7 \mathrm{mmol} / \mathrm{L}$; (3) HDL cholesterol level $<1.03 \mathrm{mmol} / \mathrm{L}$ for men and $<1.30 \mathrm{mmol} / \mathrm{L}$ for women; (4) fasting glucose level $\geq 5.6 \mathrm{mmol} / \mathrm{L}$ or use of antidiabetic medications (insulin or oral agents); or (5) systolic blood pressure $\geq 130 \mathrm{mmHg}$ and/or diastolic blood pressure $\geq 85 \mathrm{mmHg}$, and/or use of antihypertensive medications. ${ }^{21}$ To determine T2DM, all participants (except those who used insulin) underwent a standardised $75 \mathrm{~g}$ oral glucose tolerance test after an overnight fast, as described elsewhere. ${ }^{20}$ According to the World Health Organization's 2006 criteria, ${ }^{22}$ participants were categorised into normal glucose metabolism (NGM), impaired fasting glucose (fasting plasma glucose $6.1-6.9 \mathrm{mmol} / \mathrm{L}$ and $2 \mathrm{~h}$ plasma glucose (after glucose load) $<7.8 \mathrm{mmol} / \mathrm{L}$ ), impaired glucose tolerance (fasting plasma glucose $<7.0 \mathrm{mmol} / \mathrm{L}$ and $2 \mathrm{~h}$ plasma glucose (after glucose load) $\geq 7.8-11.1 \mathrm{mmol} / \mathrm{L}$ ), or T2DM (fasting plasma glucose $\geq 7.0 \mathrm{mmol} / \mathrm{L}$ or $2 \mathrm{~h}$ plasma glucose (after glucose load) $\geq 11.1 \mathrm{mmol} / \mathrm{L}$ ). Participants on diabetes medication and without type 1 diabetes were also considered to have T2DM. Impaired fasting glucose and impaired glucose tolerance were combined into impaired glucose metabolism (IGM). For the analyses with T2DM as outcome measure, we used the categories: having T2DM and not having T2DM (NGM and IGM).

\section{Accelerometry data}

Daily activity levels were measured using the activPAL3 ${ }^{\mathrm{TM}}$ physical activity monitor (PAL Technologies, Glasgow, UK). The activPAL3 is a small $(53 \times 35 \times 7 \mathrm{~mm})$, lightweight 
(15 g) triaxial accelerometer that records movement in the vertical, anteroposterior and mediolateral axes, and also determines posture (sitting or lying, standing and stepping) based on acceleration information. The device was attached directly to the skin on the front of the right thigh with transparent $3 \mathrm{M}$ Tegaderm ${ }^{\mathrm{TM}}$ tape, after the device had been waterproofed using a nitrile sleeve. Participants were asked to wear the accelerometer for 8 consecutive days, without removing it at any time. To avoid inaccurately identifying non-wear time, participants were asked not to replace the device once removed. Data were uploaded using the activPAL software and processed using customised software written in MATLAB R2013b (MathWorks, Natick, MA, USA). Data from the first day were excluded from the analysis because participants performed physical function tests at the research centre after the device was attached. In addition, data from the final wear day providing $\leq 14$ waking hours of data were excluded from the analysis. Participants were included if they provided at least 1 valid day ( $\geq 10 \mathrm{~h}$ of waking data).

The total amount of sedentary time was based on the sedentary posture (sitting or lying), and calculated as the mean time spent in a sedentary position during waking time per day. The method used to determine waking time has been described elsewhere. ${ }^{23}$ The total amount of standing time was based on the standing posture, and calculated as the mean time spent standing during waking time per day. The total amount of stepping was based on the stepping posture, and calculated as the mean time stepping during waking time per day.

\section{Covariates}

Covariates which were extracted from a questionnaire included sex, age, level of education, smoking status, alcohol consumption, energy intake, mobility limitation, and prevalent cardiovascular disease. Level of education was categorized into low, medium and high, and smoking status into never, former and current smoker. Alcohol consumption was categorized into non-consumers, low consumers ( $\leq 7$ glasses per week for women and $\leq 14$ glasses per week for men), and high consumers (>7 glasses per week for women and $>14$ glasses per week for men). Energy intake was obtained from a food frequency questionnaire and calculated as the mean energy intake (kcal) per day. Mobility limitation was obtained from the Short Form Health Survey (SF-36) questionnaire and was defined as having difficulty walking $500 \mathrm{~m}$ or climbing the stairs. Prevalent CVD was defined as a self-reported history of myocardial infarction, cerebrovascular infarction or haemorrhage, or percutaneous artery angioplasty of, or vascular surgery on, the coronary, abdominal, 
peripheral or carotid arteries. The use of lipid-modifying, antihypertensive and glucose-lowering medication was assessed during a medication interview. Depression was assessed by the Mini-International Neuropsychiatric Interview (MINI). ${ }^{24}$ Lastly, glucose metabolism status, using the three categories NGM, IGM, T2DM, was used as a covariate.

\section{Statistical analysis}

Characteristics of the total study population and according to sex-specific tertiles (to obtain equal distributions of men and women) of sedentary time were summarized as means with standard deviations (SD) or as numbers and percentages. Non-normally distributed variables were described using the median and the interquartile range.

Associations of $30 \mathrm{~min}$ of sedentary time, standing, and stepping with the cardio-metabolic outcomes were examined for each type of behaviour and each outcome separately, using linear regression analysis for the continuous variables and logistic regression analysis for the metabolic syndrome and T2DM. In models 1 the associations were adjusted for sex, age, level of education, waking time, and glucose metabolism status (the association with the metabolic syndrome and T2DM were not adjusted for glucose metabolism status). In models 2 the associations were additionally adjusted for smoking status, alcohol consumption, energy intake, mobility limitation, prevalent cardiovascular disease, depression, and antihypertensive and lipid-modifying medication (the metabolic syndrome and T2DM were not adjusted for medication use). All models 2, except those describing waist circumference, $\mathrm{BMI}$, and the metabolic syndrome also included $\mathrm{BMI}$ as covariate.

The ISM approach was applied to the cardio-metabolic outcomes that were statistically significantly associated in the regression analyses (model 2). The analysis involves a linear regression model that includes all types of behaviour (sedentary, standing, and stepping) and total waking time. By dropping one type of behaviour, e.g. sedentary behaviour, the coefficients of another type of behaviour, e.g. standing, will represent the estimated effect of replacing 30 min sedentary time with 30 min standing. The following estimated replacement effects were modelled: sedentary time replaced by standing and stepping, and standing replaced by stepping. We used time blocks of 30 min because a minimum of $30 \mathrm{~min}$ high intensity physical activity per day is required to meet the physical activity guidelines. ${ }^{25}$ The associations were adjusted as described before. In all models the assumption of linearity was verified and multi-collinearity was not indicated (variance-inflating factors were $<2.5$ ). 
In additional analyses, we tested for interaction between all types of behaviour (sedentary time, standing, and stepping) and glucose metabolism status. A P-value of $<0.10$ was considered statistically significant. Further, the linear regression analyses were repeated with replacement of office blood pressure with $24 \mathrm{~h}$ average ambulatory blood pressure $(n=1,956)$. All analyses were conducted with IBM SPSS Statistics 22.0 (IBM Corp., Armonk, NY, USA).

\section{RESULTS}

A total of 2,213 participants (51\% men) with an average age of $60.0 \pm 8.1$ years were included. They provided on average $6.3 \pm 1.2$ valid days of data, although most participants (57\%) provided 7 valid days. Average waking time was $15.7 \pm 0.9 \mathrm{~h}$ per day, of which $9.4 \pm 1.7 \mathrm{~h}(60 \%)$ was spent in a sedentary position. The remaining waking hours were spent standing $(4.3 \pm 1.3 \mathrm{~h} ; 27 \%)$ and stepping $(2.0 \pm 0.7 \mathrm{~h}) ; 13 \%)$. Characteristics of the total study population and according to sex-specific tertiles of sedentary time are presented in Table 6.1.

Table 6.2 presents associations of sedentary time, standing, and stepping with cardiometabolic outcomes. Sedentary time accumulated in bouts of 30 min was statistically significantly associated with a larger waist circumference, a higher BMI, a lower HDL cholesterol level, a higher total-to-HDL cholesterol ratio, a higher triglyceride level, a higher $2 \mathrm{~h}$ post-load glucose level, and a higher fasting insulin level (models 2). Standing (30 $\mathrm{min} /$ day) was statistically significantly associated with a lower waist circumference, a lower BMI, a higher HDL cholesterol level, a lower total-to-HDL cholesterol ratio, a lower triglyceride level and a lower fasting insulin level in models 2 . Similar results were seen for 30 min of stepping per day, although stepping was also significantly associated with a lower $2 \mathrm{~h}$ post-load glucose level. No statistically significant associations were observed between any of the types of behaviour and blood pressure, fasting glucose levels, and $\mathrm{HbAlc}$ in models 2 . In contrast, for all types of behaviour statistically significant associations were seen (in model 2 ) with the metabolic syndrome $\left(O R_{\text {sedentary }}=1.15 ;(95 \%\right.$ $\mathrm{Cl}=1.12 ; 1.19) ; \mathrm{OR}_{\text {standing }}=0.88 ;(0.85 ; 0.92) ; \mathrm{OR}_{\text {stepping }}=0.69 ;(0.64 ; 0.75) ;$ data not tabulated) and T2DM $\left(O R_{\text {sedentary }}=1.12 ;(1.07 ; 1.16) ; O R_{\text {standing }}=0.91 ;(0.86 ; 0.95)\right.$; $\mathrm{OR}_{\text {stepping }}=0.76 ;(0.69 ; 0.84)$; data not tabulated). 


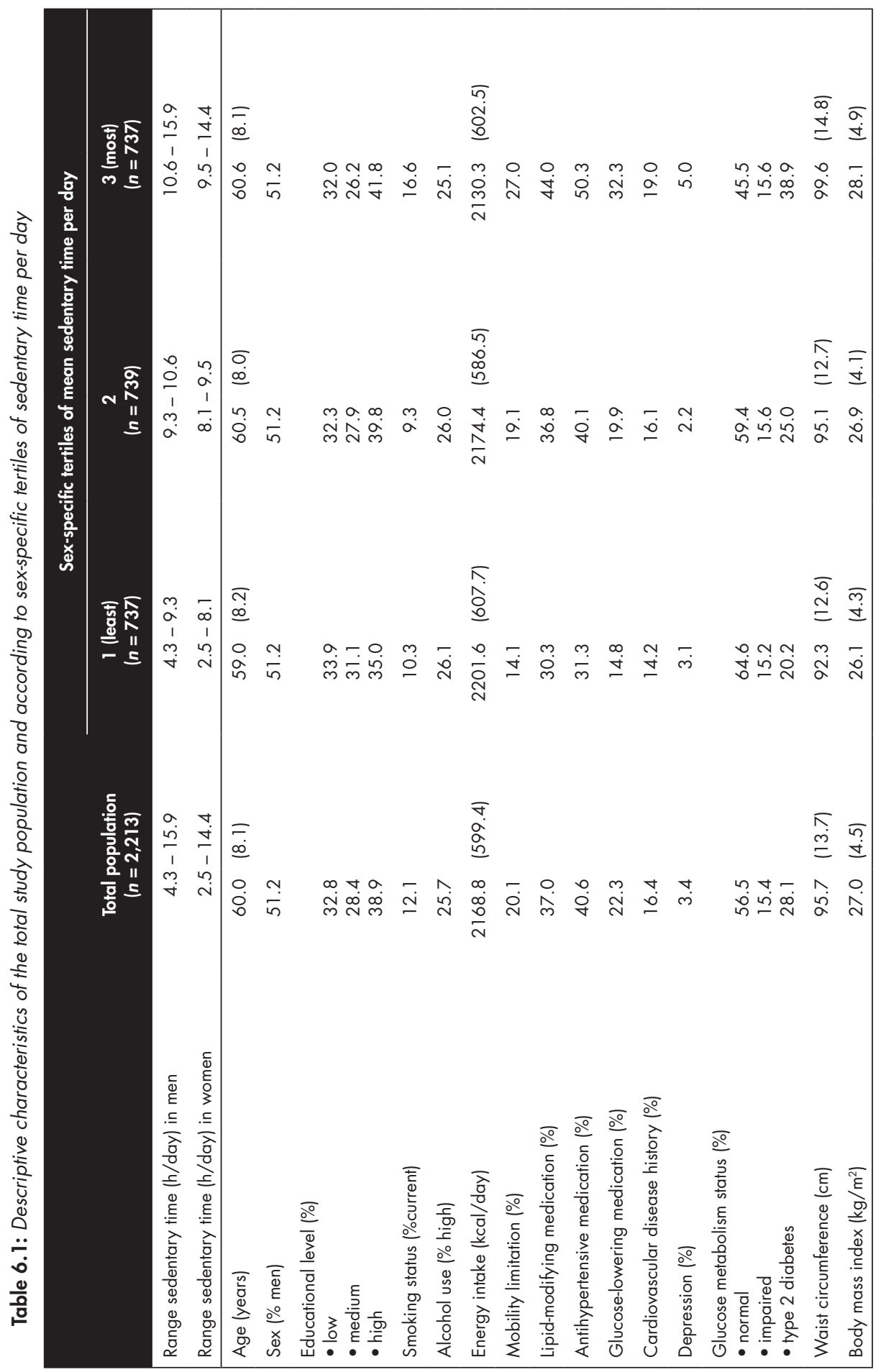




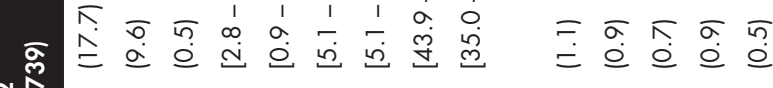

峁

$\stackrel{\therefore}{\check{0}}$ 


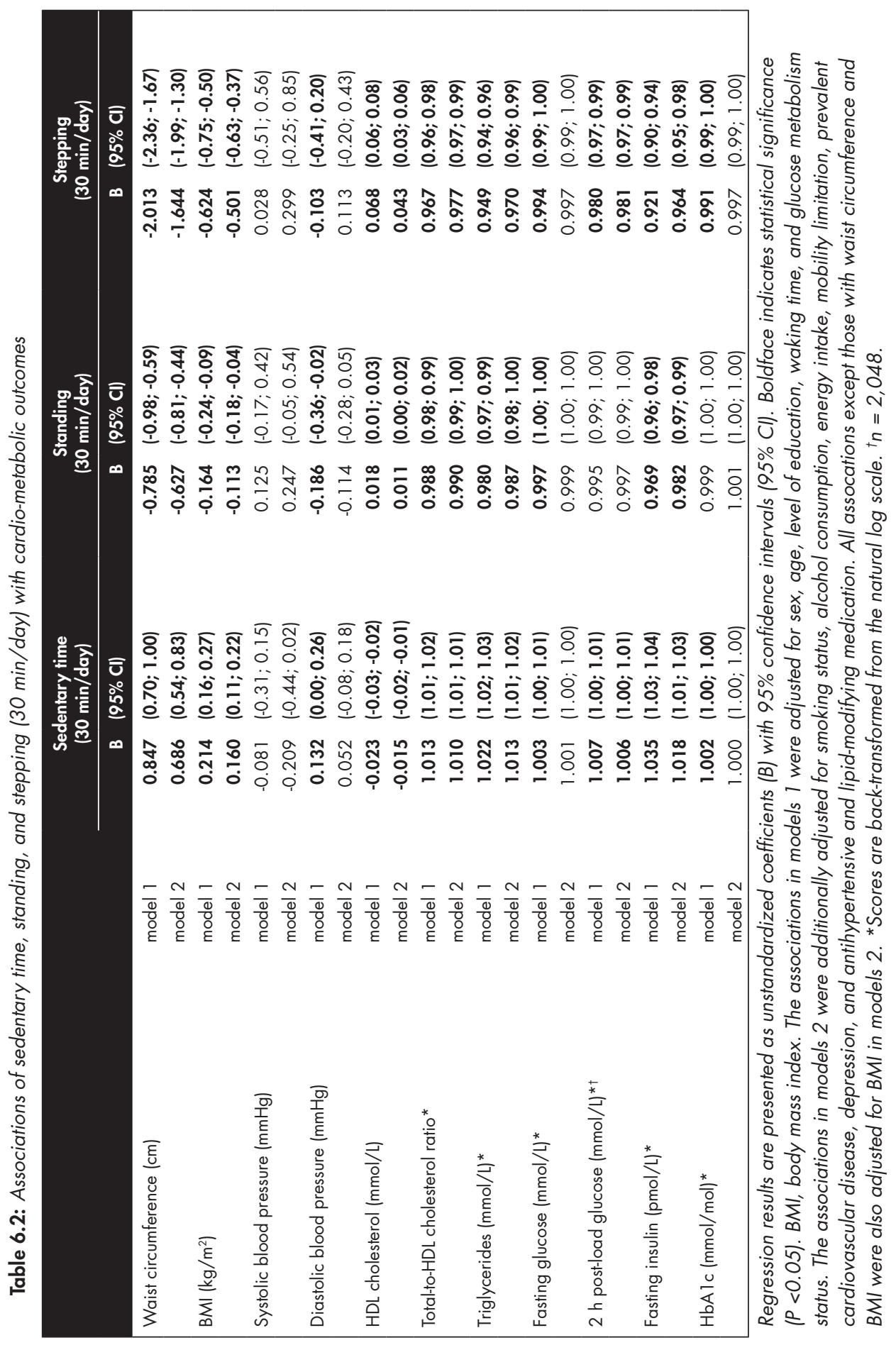


Table 6.3 presents the estimated effects of replacing $30 \mathrm{~min}$ of one type of behaviour with another. After adjustment for confounders, a daily replacement of $30 \mathrm{~min}$ of sedentary time with 30 min of standing was associated with a $0.41 \mathrm{~cm}\left(B_{\text {sedentary-standing }}\right.$ $=-0.41 ;(-0.60 ;-0.21))$ smaller waist circumference, while a replacement with stepping was associated with a $1.42 \mathrm{~cm}\left(B_{\text {sedentary-stepping }}=-1.42 ;(-1.78 ;-1.06)\right)$ smaller waist circumference. Replacing standing by stepping was, after adjustment for confounders, associated with a $1.02 \mathrm{~cm}\left(B_{\text {standing-stepping }}=-1.02 ;(-1.47 ;-0.56)\right)$ smaller waist circumference. After adjustment for confounders, statistically significant associations with a lower $\mathrm{BMI}$ were seen when stepping was the replacement of sedentary time $\left(\mathrm{B}_{\text {sedentary- }}\right.$ stepping $=-0.48 ;(-0.62 ;-0.35))$ or standing $\left(B_{\text {standing-stepping }}=-0.44 ;(-0.61 ;-0.27)\right)$. This was also seen for HDL cholesterol: reducing sedentary time or standing with $30 \mathrm{~min} /$ day was, after adjustment for confounders, statistically significantly associated with a higher HDL cholesterol level when it was replaced by equal amounts of stepping $\left(B_{\text {sedentary-stepping }}=0.04\right.$; $\left.(0.03 ; 0.05) ; B_{\text {standing-stepping }}=0.04 ;(0.02 ; 0.05)\right)$. For total-to-HDL cholesterol ratio and triglycerides levels, only reallocating sedentary time to standing or stepping resulted in lower total-to-HDL ratios $\left(\mathrm{B}_{\text {sedentary-standing }}=0.99 ;(0.99 ; 1.00) ; \mathrm{B}_{\text {sedentary-stepping }}=0.98 ;(0.97\right.$; 0.99); back transformed from log scale) and lower triglyceride levels $\left(B_{\text {sedentary-standing }}=0.99\right.$; $(0.98,1.00) ; B_{\text {sedentary-stepping }}=0.98 ;(0.96,0.99) ;$ back transformed from log scale) after adjustment for confounders. A statistically significant association with a lower $2 \mathrm{~h}$ post-load glucose level was seen when 30 min of sedentary time or 30 min of standing were replaced by 30 min of stepping $\left(B_{\text {sedentary-stepping }}=0.98 ;(0.97 ; 0.99) ; B_{\text {standing-stepping }}=0.98\right.$; (0.97; 0.99); back transformed from log scale). Further, when 30 min of sedentary time were replaced by $30 \mathrm{~min}$ of standing or stepping, it resulted in a statistically significantly lower fasting insulin level $\left(B_{\text {sedentary-standing }}=0.99 ;(0.98 ; 1.00) ; B_{\text {sedentary-stepping }}=0.97 ;(0.95\right.$, 0.99); back-transformed from log-scale). No statistically significant associations with fasting insulin were seen when replacing standing with stepping. 
Table 6.3: Estimated effects of replacing 30 min sedentary time or standing with 30 min standing or stepping on cardio-metabolic outcomes using isotemporal substitution models

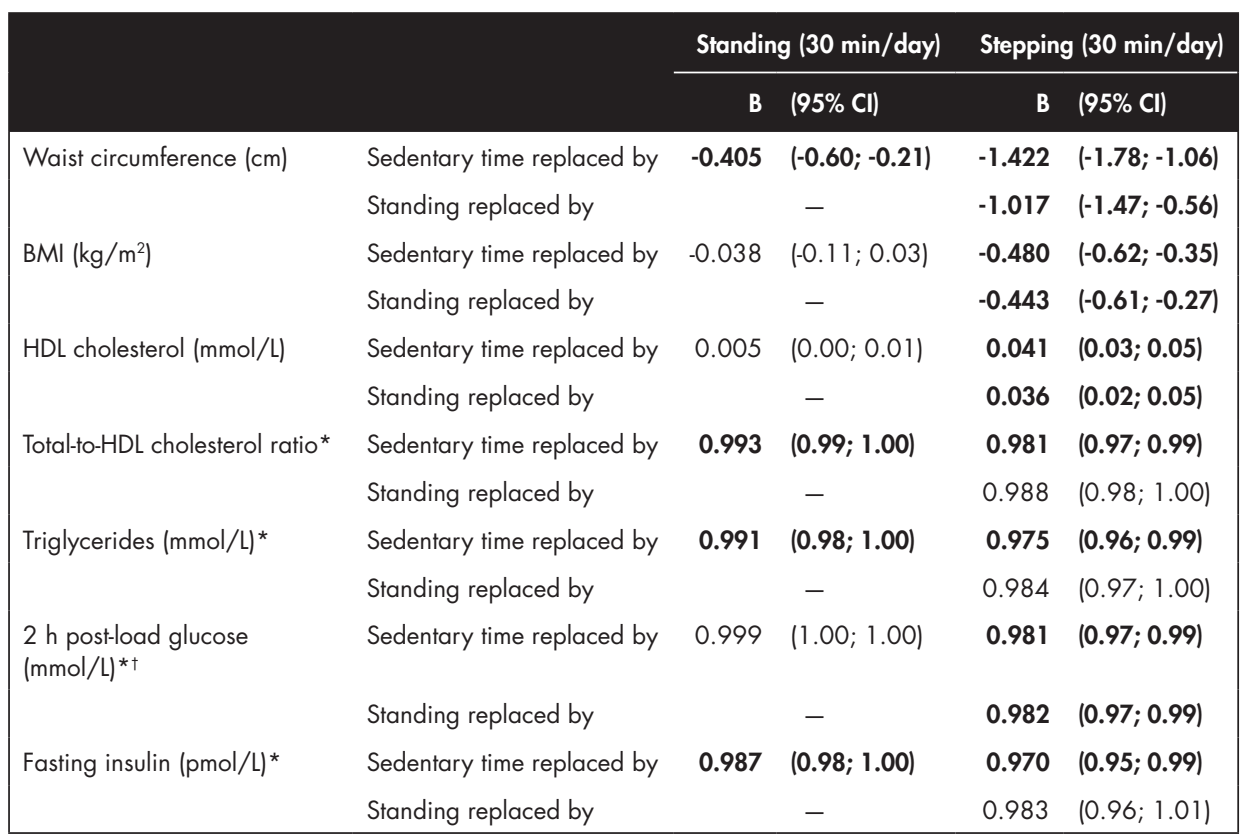

Regression results are presented as unstandardized coefficients (B) with $95 \%$ confidence intervals (95\% Cl). Boldface indicates statistical significance $(P<0.05)$. BMl, body mass index. Associations were adjusted for sex, age, level of education, waking time, glucose metabolism status, smoking status, alcohol consumption, energy intake, mobility limitation, prevalent cardiovascular disease, depression, and antihypertensive and lipid-modifying medication. All associations except those with waist circumference and $B M I$ were also adjusted for BMI. * Scores are back-transformed from the natural log scale. ${ }^{t} n=2,048$.

Figure 6.1 presents the estimated effects on the metabolic syndrome and T2DM of replacing $30 \mathrm{~min}$ of one type of behaviour with another. After adjustment for confounders, statistically significant associations with the metabolic syndrome were seen when sedentary time was replaced by either standing $\left(O R_{\text {sedentary-standing }}=0.93 ;(0.89 ; 0.97)\right)$, or stepping $\left(O R_{\text {sedentary-stepping }}=0.72 ;(0.66 ; 0.78)\right)$, and when standing was replaced by stepping $\left(O R_{\text {standing-stepping }}=0.77 ;(0.70 ; 0.86)\right)$. Similar results were seen for T2DM; after adjustment for confounders, statistically significant associated were seen when sedentary time was replaced by standing $\left(O R_{\text {sedentary-standing }}=0.94 ;(0.90 ; 0.99)\right)$, or stepping $\left(O R_{\text {sedentary-stepping }}=0.79 ;(0.72 ; 0.87)\right)$, and when standing was replaced by stepping $\left(O R_{\text {standing-stepping }}=0.84 ;(0.74 ; 0.95)\right)$. 


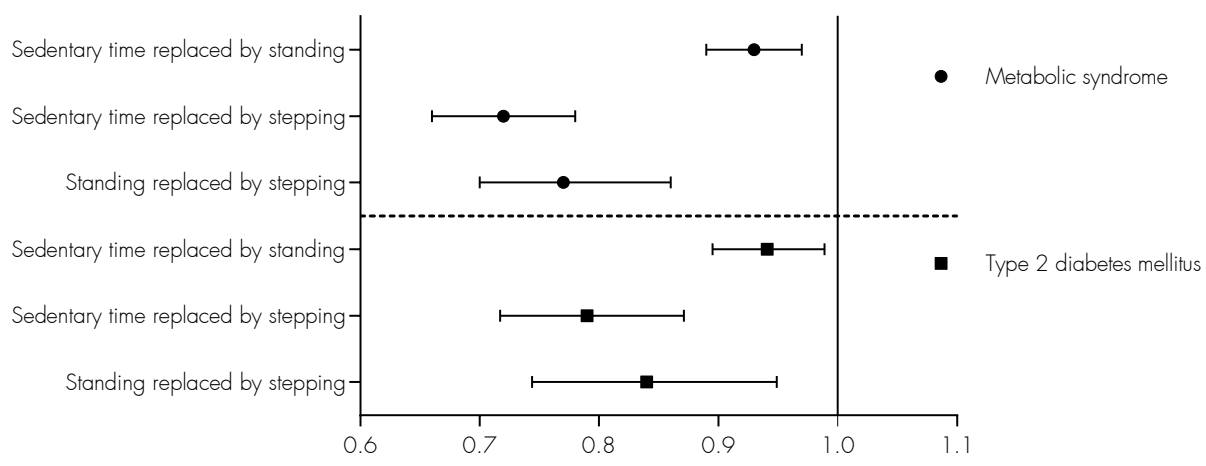

Odds ratio with $95 \%$ confidence interval

Figure 6.1: Estimated effects of replacing 30 min of sedentary time or standing with 30 min of standing or stepping on the metabolic syndrome and type 2 diabetes mellitus, using isotemporal substitution models. Associations were adjusted for sex, age, level of education, waking time, smoking status, alcohol consumption, energy intake, mobility limitation, prevalent cardiovascular disease, and depression. The assocation with type 2 diabetes mellitus was also adjusted for body mass index.

Interaction terms between stepping and glucose metabolism status were statistically significant for waist circumference, BMI, and fasting insulin (all $P<0.10$ ), therefore the replacement analyses were repeated according to glucose metabolism status. Figure 6.2 presents the estimated effects on waist circumference and BMI of replacing $30 \mathrm{~min}$ of sedentary time with equal amounts of standing or stepping, in the NGM, IGM and T2DM groups. Replacing sedentary time with standing as well as stepping was statistically significantly associated with a smaller waist circumference, but larger effects were seen in the IGM and T2DM groups compared to the NGM group. For example, replacing sedentary time with stepping was associated with a $1.05 \mathrm{~cm}\left(B_{\text {sedentary-stepping }}=-1.05 ;(-1.47 ;-0.63)\right)$ smaller waist circumference in the NGM group, while this was $1.70 \mathrm{~cm}\left(\mathrm{~B}_{\text {sedentary-stepping }}=\right.$ $-1.70 ;(-2.67 ;-0.74))$ and $1.89 \mathrm{~cm}\left(B_{\text {sedentary-stepping }}=-1.89 ;(-2.73 ;-1.06)\right)$ in the IGM and T2DM groups respectively. A similar pattern was seen for BMI: replacing sedentary time with stepping was statistically significantly associated with a $0.36 \mathrm{~kg} / \mathrm{m}^{2}\left(B_{\text {sedentary-stepping }}=-0.36\right.$; $(-0.51 ;-0.20))$ lower BMI in the NGM group, while this was $0.62 \mathrm{~kg} / \mathrm{m}^{2}\left(\mathrm{~B}_{\text {sedentary-stepping }}=\right.$ $-0.62 ;(-0.98 ;-0.26))$ in the IGM and $0.62 \mathrm{~kg} / \mathrm{m}^{2}$ (B $\left.B_{\text {sedentary-stepping }}=-0.62 ;(-0.95 ;-0.30)\right)$ in the T2DM groups. Although a statistically significant interaction term was found between stepping and glucose metabolism status for fasting insulin, the replacement effects on fasting insulin in the NGM, IGM, and T2DM groups were similar (data not shown). 


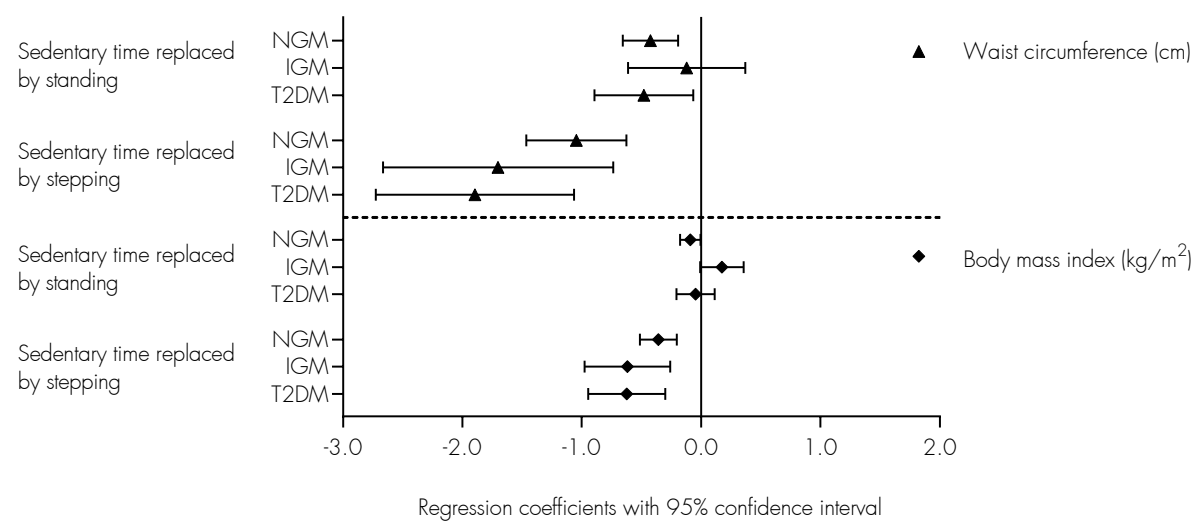

Figure 6.2: Estimated effects of replacing 30 min of sedentary time with 30 min of standing or stepping on waist circumference and body mass index, according to glucose metabolism status, using isotemporal substitution models. NGM, normal glucose metabolism; IGM impaired glucose metabolism; T2DM, type 2 diabetes mellitus. Associations were adjusted for sex, age, level of education, waking time, smoking status, alcohol consumption, energy intake, mobility limitation, prevalent cardiovascular disease, depression, and antihypertensive and lipid-modifying medication.

Additional analysis using $24 \mathrm{~h}$ average ambulatory blood pressure instead of office blood pressure resulted in similar findings; no associations were seen with any type of behaviour (data not tabulated). Further, results were similar when we excluded participants with $<4$ valid days ( $\geq 10 \mathrm{~h}$ of waking data).

\section{DISCUSSION}

In this study we used the ISM approach to estimate the effects of a theoretical reallocation of sedentary behaviour (sitting or lying) to standing or stepping with cardio-metabolic outcomes, the metabolic syndrome and T2DM. The results show that, after adjustment for confounders, a replacement of sedentary behaviour with non-sedentary behaviour was statistically significantly associated with beneficial metabolic outcomes. The largest estimated effects were seen when $30 \mathrm{~min}$ of sedentary time were replaced by $30 \mathrm{~min}$ of stepping, as this resulted in a $1.4 \mathrm{~cm}$ smaller waist circumference, a $0.5 \mathrm{~kg} / \mathrm{m}^{2}$ lower BMI, and improved levels of cholesterol (both HDL and total-to-HDL ratio), triglyceride, $2 \mathrm{~h}$ post-load glucose, and fasting insulin. In addition, the replacement of $30 \mathrm{~min}$ of sedentary 
behaviour with 30 min of standing was associated with a reduction in waist circumference, a lower total-to-HDL cholesterol ratio, a lower triglyceride level, and a lower insulin level. Further, reducing standing by replacing it with stepping was statistically significantly associated with reductions in waist circumference and BMI, and improved HDL cholesterol and $2 \mathrm{~h}$ post-load glucose levels. Also, reallocating sedentary time to standing or stepping, and reallocating standing to stepping was significantly associated with a lower odds for the metabolic syndrome and T2DM. Finally, when estimating the effects of replacing sedentary time with standing or stepping in groups according to glucose metabolism status, we saw larger replacement effects in participants with IGM and T2DM compared to participants with NGM for both waist circumference and BMI, although this was likely due to the larger measures of waist circumference and BMI in the IGM and T2DM groups rather than glucose metabolism per se.

Taken together, the results suggest that reallocating sedentary behaviour, even in small amounts of $30 \mathrm{~min} /$ day, may have favourable effects on metabolic outcomes, the metabolic syndrome, and T2DM. Interestingly, these favourable results were not only seen when sedentary time was replaced by stepping (e.g. physical activity), but also when it was replaced by standing. Also, as stronger associations for waist circumference and BMI were seen in participants with IGM and T2DM, reallocation of only 30 min of sedentary time per day to standing or stepping may be clinically relevant and feasible. Thus, our results provide new insight in the potential effects of reallocating sedentary time which may be used for health guidelines and may also give direction to experimental or intervention studies on the amounts of reallocation time that should be examined. For these reasons, our findings may be important; for the general population, for those who cannot participate in high intensity activities and for those who cannot meet the physical activity guidelines.

Our results are in line with findings of other ISM studies. Most of these studies reported associations of reallocating sedentary time to LPA or MVPA with reductions in waist circumference ${ }^{10,12-15}$ and BMI. ${ }^{10-13}$ Less consistent results were seen for other metabolic outcomes including levels of cholesterol, triglycerides, and glucose. In a few studies including ours, ${ }^{13,15}$ statistically significant associations were seen with HDL cholesterol when replacing sedentary time with more active types of behaviour, while other studies showed no associations ${ }^{10}$ or only associations with MVPA. ${ }^{11,14}$ Similar inconsistencies were seen for triglycerides, ${ }^{11,13-15}$ and glucose levels. ${ }^{13-16}$ These inconsistencies could be due to the study population which varied in age (36 to 80 years) and in metabolic profile (healthy participants, participants at increased risk for T2DM, participants with T2DM). In 
addition, the replacement time varied between studies, as some studies used blocks of 10 $\min ,{ }^{11,15}$ while we and others used 30 min blocks, ${ }^{12,14,16}$ and even $2 \mathrm{~h}$ blocks were used. ${ }^{13}$ Further, most studies used a waist- or hip-worn accelerometer which cannot distinguish between postures. Consequently, estimations of the amount of sedentary time in these studies were less accurate than the estimates in our study, as we used a posture-based accelerometer. ${ }^{17-19}$ In only one other study a posture-based accelerometer was used and the reported results were similar to ours, although in that study an allocation time of 2 hours was used. ${ }^{13}$

Possible pathophysiological mechanisms that may explain findings have not yet been studied extensively. However, studies in animals have described plausible mechanisms that may underlie the detrimental health effects of large amounts of sedentary time, including reductions in lipoprotein lipase (LPL) and lipid phosphate phosphatase-1 (LPP1) due to muscle inactivity. ${ }^{26,27}$ Participation in non-sedentary behaviour may be sufficient to counterbalance these processes. Additionally, previous experimental and epidemiological studies have demonstrated that breaking up sedentary time with non-sedentary time had beneficial effects on metabolic outcomes, which supports this hypothesis. ${ }^{28}$ Nevertheless, physiological studies are needed to further examine this.

The use of posture-based accelerometer was a key strength of this study, as well as the large study population consisting of adults with NGM, IGM, and T2DM. However, a few limitations should also be mentioned. Due to the cross-sectional nature of the study, causal relationships could not be determined. Thus, the replacement effects resulting from the ISM approach were estimated rather than based on actual behavioural reallocation. Although this method can provide valuable insights in the potential effects on health, experimental and intervention studies are warranted to examine the actual behavioural reallocation effects on both short- and long-term. Further, as our accelerometry data were posture-based, we used total stepping time as a measure for physical activity, but different intensity levels could not be determined. Therefore, future studies ideally should combine posture-based data for accurate assessment of sedentary behaviour with acceleration data for determining of physical activity intensity levels. In addition, due to missing data, we had to exclude 1,200 participants. However, the excluded participants did not differ from our study sample with regard to demographic factors, accelerometry variables and outcomes measures (data not shown). Finally, our study population consisted of a relatively healthy population with well-controlled individuals with T2DM; therefore the result might not representative for the general population of adults aged $40-75$ years. 
To conclude, our study demonstrated that a theoretical replacement of sedentary time with standing or stepping was associated with reductions in waist circumference and BMI, improved levels of HDL cholesterol, triglycerides, glucose and insulin, and a decreased likelihood for the metabolic syndrome and T2DM. In addition, replacing standing with stepping was associated with an improved waist circumference, BMl, $\mathrm{HDL}$ cholesterol and glucose levels, the metabolic syndrome and T2DM. Further, larger theoretical replacement effects for both waist circumference and BMI were seen in participants with IGM and T2DM. As reallocating of even small amounts of sedentary behaviour to any type of non-sedentary behaviour seems to be beneficial for health, consideration should be given to developing health guidelines that include recommendations for the daily reallocation of sedentary time, although longitudinal studies on actual behavioural reallocation effects are needed to confirm our results. 


\section{REFERENCES}

1. Sedentary Behaviour Research Network. Standardized use of the terms "sedentary" and "sedentary behaviours." Appl Physiol Nutr Metab. 2012;37:540-2.

2. Brocklebank LA, Falconer CL, Page AS, Perry R, Cooper AR. Accelerometer-measured sedentary time and cardiometabolic biomarkers: A systematic review. Prev Med. 2015;76:92-102.

3. Edwardson CL, Gorely T, Davies M, Gray W, Khunti K, Wilmot EG, et al. Association of sedentary behaviour with metabolic syndrome: A meta-analysis. PLoS One. 2012;7(4):e34916.

4. Biswas A, Oh PI, Faulkner GE, Bajaj RR, Silver $M A$, Mitchell MS, et al. Sedentary time and its association with risk for disease incidence, mortality, and hospitalization in adults. Ann Intern Med. 2015;162(10):123-32.

5. Wilmot EG, Edwardson $\mathrm{CL}$, Achana FA, Davies M, Gorely T, Gray L, et al. Sedentary time in adults and the association with diabetes, cardiovascular disease and death: Systematic review and meta-analysis. Diabetologia. 2012;55(1 1):2895-905.

6. Department of Health Physical Activity Health Improvement and Protection. Start Active, Stay Active. Report. 2011. Available from: https:// www.gov.uk/government/publications/startactive-stay-active-a-report-on-physical-activity-fromthe-four-home-countries-chief-medical-officers

7. Canadian Society of Exercise Physiology. Canadian Physical Activity and Sedentary Behaviour Guidelines. 2012. Available from: www.csep.ca/guidelines

8. Australian Government Department of Health. Australia's Physical Activity and Sedentary Behaviour Guidelines For Adults (18-64 years). 2014. Available from: http://www.health.gov. au/internet/main/publishing.nsf/Content/phyactivity

9. Mekary RA, Willett WC, Hu FB, Ding EL. Isotemporal substitution paradigm for physical activity epidemiology and weight change. Am J Epidemiol. 2009;170(4):519-27.

10. Falconer CL, Page AS, Andrews RC, Cooper AR. The potential impact of displacing sedentary time in adults with type 2 diabetes. Med Sci Sport Exerc. 2015;47(10):2070-5.

11. Hamer M, Stamatakis E, Steptoe A. Effects of substituting sedentary time with physical activity on metabolicrisk. Med Sci Sport Exerc. 2014;46(10):1946-50.

12. Healy $G N$, Winkler EAH, Brakenridge $C L$, Reeves MM, Eakin EG. Accelerometer-derived sedentary and physical activity time in overweight/obese adults with type 2 diabetes: Cross-sectional associations with cardiometabolic biomarkers. PLoS One. 2015;10:e0119140.

13. Healy GN, Winkler EAH, Owen N, Anuradha $S$, Dunstan DW. Replacing sitting time with standing or stepping : Associations with cardio-metabolic risk biomarkers. Eur Heart J. 2015; 14(36):2643-9.

14. Buman MP, Winkler EAH, Kurka JM, Hekler EB, Baldwin $C M$, Owen N, et al. Reallocating time to sleep, sedentary behaviors, or active behaviors: Associations with cardiovascular disease risk biomarkers, NHANES 2005-2006. Am J Epidemiol. 2013;179(3):323-34.

15. Ekblom-Bak E, Ekblom O, Bergstro $m \mathrm{G}$, Bo riesson $M$. Isotemporal substitution of sedentary time by physical activity of different intensities and bout lengths, and its associations with metabolic risk. Eur J Prev Cardiol. 2015;3. Epub ahead of print.

16. Yates T, Henson J, Edwardson C, Dunstan $\mathrm{D}$, Bodicoat $\mathrm{DH}$, Khunti K, et al. Objectively measured sedentary time and associations with insulin sensitivity: Importance of reallocating sedentary time to physical activity. Prev Med. 2015;76:79-83.

17. Lyden K, Kozey Keadle SL, Staudenmayer JW, Freedson PS. Validity of two wearable monitors to estimate breaks from sedentary time. Med Sci Sports Exerc. 2012;44(1 1):2243-52.

18. Kozey-Keadle S, Libertine A, Lyden K, Staudenmayer J, Freedson PS. Validation of wearable monitors for assessing sedentary behavior. Med Sci Sport Exerc. 2011;43(8):1561-7.

19. Godfrey A, Culhane KM, Lyons GM. Comparison of the performance of the activPAL ${ }^{T M}$ Professional physical activity logger to a discrete accelerometer-based activity monitor. Med Eng Phys. 2007;29(8):930-4. 
20. Schram MT, Sep SJS, van der Kallen CJ, Dagnelie PC, Koster A, Schaper N, et al. The Maastricht Study: An extensive phenotyping study on determinants of type 2 diabetes, its complications and its comorbidities. Eur J Epidemiol. 2014;29(6):439-51

21. Alberti KGMM, Eckel RH, Grundy SM, Zimmet PZ, Cleeman JI, Donato K a., et al. Harmonizing the metabolic syndrome. Circulation. 2009; 120(16):1640-5.

22. World Health Organization. Definition and Diagnosis of Diabetes Mellitus and Intermediate Hyperglycemia. World Health Organization. 2006.

23. Van der Berg J, Willems $P$, Van der Velde J, Savelberg $H$, Schaper N, Schram M, et al. Identifying waking time in 24-h accelerometry data in adults using an automated algorithm. J Sports Sci. 2016;2:1-7.

24. Sheehan DV, Lecrubier $Y$, Sheehan KH, Amorim P, Janavs J, Weiller E, et al. The Mini-International Neuropsychiatric Interview (M.I.N.I.): The development and validation of a structured diagnostic psychiatric interview for DSM-IV and ICD-10. J Clin Psychiatry. 1998;59(Suppl 20):22-3.

25. Hildebrandt $H$, Ooijendijk $M$, Hopman $M$. Trendrapport Bewegen en Gezondheid. TNO Kwaliteit van Leven. 2010.

26. Hamilton M, Hamilton D, Zderic T. Role of low energy expenditure and sitting in obesity, metabolic syndrome, type 2 diabetes, and cardiovascular disease. Diabetes. 2007;56:2655-67.

27. Hamilton MT, Hamilton DG, Zderic TW. Sedentary Behavior as a mediator of type 2 diabetes. Diabetes and Physical Activity. 2014. p. 11-26.

28. Benatti F, Ried-Larsen $M$. The effects of breaking up prolonged sitting time. Med Sci Sport Exerc. 2015;47(10):2053-61. 

Chapt

照 


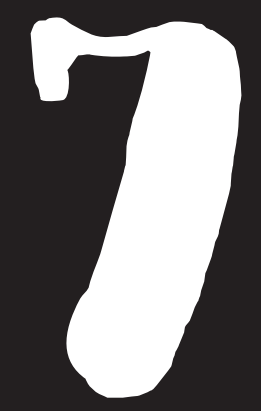

Associations of total amount and patterns of sedentary behaviour with type 2 diabetes and the metabolic syndrome: The Maastricht Study

JULIANNE D. VAN DER BERG | COEN D.A. STEHOUWER | HANS BOSMA | JEROEN H.P.M. VAN DER VELDE | PAUL J.B. WILLEMS | HANS H.C.M. SAVELBERG | MIRANDA T. SCHRAM | SIMONE J.S. SEP | CARLA J.H. VAN DER KALLEN | RONALD M.A. HENRY | PIETER C. DAGNELIE | NICOLAAS C. SCHAPER | ANNEMARIE KOSTER 


\section{AB STR ACT}

Aims/hypothesis: The study investigated cross-sectional associations of total amount and patterns of sedentary behaviour with glucose metabolism status and the metabolic syndrome.

Methods: We included 2,497 participants (52\% men, age (mean $\pm S D$ ): $60.0 \pm 8.1$ years) from The Maastricht Study who were asked to wear an activPAL3 accelerometer $24 \mathrm{~h} /$ day for 8 consecutive days. We calculated the daily amount of sedentary time, daily number of sedentary breaks and prolonged sedentary bouts ( $\geq 30 \mathrm{~min}$ ), and the average duration of the sedentary bouts. To determine glucose metabolism status, participants underwent an oral glucose tolerance test. Associations of sedentary behaviour variables with glucose metabolism status and the metabolic syndrome were examined using multinomial logistic regression analyses.

Results: Overall, 1,395 (55.9\%) participants had normal glucose metabolism, 388 (15.5\%) had impaired glucose metabolism and 714 (28.6\%) had type 2 diabetes (T2DM). The odds ratio per additional hour of sedentary time was $1.22(95 \% \mathrm{Cl}=1.13 ; 1.32)$ for T2DM and $1.39(1.27 ; 1.53)$ for the metabolic syndrome. No significant or only weak associations were seen for the number of sedentary breaks, number of prolonged sedentary bouts or average bout duration with either glucose metabolism status or the metabolic syndrome.

Conclusions/interpretation: An extra hour of sedentary time was associated with a $22 \%$ increased odds for T2DM and a $39 \%$ increased odds for the metabolic syndrome. The pattern in which sedentary time was accumulated was weakly associated with the presence of the metabolic syndrome. These results suggest that sedentary behaviour may play a significant role in the development and prevention of T2DM, although longitudinal studies are needed to confirm our findings. 


\section{INTRODUCTION}

Type 2 diabetes mellitus is a chronic disease with high prevalence and incidence worldwide that, next to its classic complications such as cardiovascular disease and retinopathy, can cluster with other chronic diseases such as dementia and chronic obstructive pulmonary disease (COPD). During the last decades several risk factors, including genetic, environmental and lifestyle factors, have been identified as relevant for the development of type 2 diabetes, ${ }^{2,3}$ but these cannot fully explain its development. Recent research interest is therefore focusing on the role of newly identified determinants, such as sedentary behaviour.

Sedentary behaviour, defined as any waking behaviour characterised by an energy expenditure $\leq 1.5$ metabolic equivalents (METs) while in a sitting or reclining position, such as watching TV or using the computer, ${ }^{4}$ can be objectively measured using an accelerometer. A number of accelerometry studies have shown unfavourable associations between total amount of sedentary time and metabolic health outcomes, including waist circumference, ${ }^{5-9}$ cholesterol and triacylglycerol levels, ${ }^{5,6,10-13}$ markers of insulin resistance, $, 5,6,9,10,13,14$ and the metabolic syndrome. ${ }^{8,11,15-17}$ Apart from the total amount of sedentary time, the pattern of sedentary time, i.e. the frequency with which sedentary time is interrupted (sedentary breaks) or the duration of uninterrupted periods of sedentary time (sedentary bouts), seems to be relevant for health outcomes. In a few studies, more sedentary breaks have been associated with better metabolic health. ${ }^{15,18,19}$

To date, large-scale studies that have objectively measured sedentary behaviour in a population with type 2 diabetes have been scarce. 5,17 However, given the large amounts of time people spend being sedentary and the high prevalence of type 2 diabetes, such studies are important. These studies can provide more insights into the associations between sedentary behaviour and diabetes, and contribute to the development of strategies to prevent diabetes and its complications and comorbidities. Therefore, we measured the total amount and patterns of sedentary behaviour with an accelerometer in a large sample of adults with type 2 diabetes (T2DM), impaired glucose metabolism (IGM) or normal glucose metabolism (NGM), who participated in The Maastricht Study. We used the thigh-worn activPAL3 accelerometer, which classifies sedentary behaviour using data on posture, as this has shown to be an accurate means of assessing sedentary behaviour. ${ }^{20,21}$ The aim of the present study was to examine associations of total amount and patterns of sedentary behaviour with glucose metabolism status and the metabolic syndrome. 


\section{METHODS}

\section{Study population}

In this study, we used data from The Maastricht Study, an observational, prospective, population-based cohort study. The rationale and methods have been described previously. ${ }^{22}$ In brief, the study focuses on the aetiology, pathophysiology, complications and comorbidities of T2DM and is characterised by an extensive phenotyping approach.

Eligible participants were individuals aged between 40 and 75 years and living in the southern part of the Netherlands. Participants were recruited through mass media campaigns and from the municipal registries and the regional Diabetes Patient Registry via mailings. Recruitment was stratified according to known type 2 diabetes status for reasons of efficiency. This study included cross-sectional data from 3,451 participants who completed the baseline survey between November 2010 and September 2013. After excluding participants who did not receive an accelerometer due to logistics ( $n=673)$, whose accelerometer measurement failed $(n=136)$ or who had other missing data $(n=145)$, a total of 2,497 participants were included in the present analyses.

The study was approved by the institutional medical ethical committee (NL3 1329.068.10) and the Minister of Health, Welfare and Sports of the Netherlands, on the basis of the Health Council's opinion (permit 131088-105234-PG). All participants gave written informed consent.

\section{Measurements}

Glucose metabolism status and the metabolic syndrome To determine glucose metabolism status, all participants (except those who used insulin) underwent a standardised $75 \mathrm{~g}$ oral glucose tolerance test after an overnight fast, as described elsewhere. ${ }^{22}$ Glucose metabolism was defined according to the World Health Organization's 2006 criteria, $^{23}$ and participants were categorised as having NGM, impaired fasting glucose (fasting plasma glucose 6.1-6.9 mmol/L and $2 \mathrm{~h}$ plasma glucose (after glucose load) $<7.8 \mathrm{mmol} / \mathrm{L}$ ), impaired glucose tolerance (fasting plasma glucose $<7.0 \mathrm{mmol} / \mathrm{L}$ and $2 \mathrm{~h}$ plasma glucose (after glucose load) $\geq 7.8-11.1 \mathrm{mmol} / \mathrm{L}$ ), or T2DM (fasting plasma glucose $\geq 7.0 \mathrm{mmol} / \mathrm{L}$ or $2 \mathrm{~h}$ plasma glucose (after glucose load) $\geq 11.1 \mathrm{mmol} / \mathrm{L}$ ). Participants on diabetes medication and without type 1 diabetes were also considered to have T2DM. For this study, we defined having either impaired fasting glucose and/or impaired glucose tolerance as IGM. 
To determine the metabolic syndrome, we measured, as described elsewhere: ${ }^{22}$ waist circumference, triacylglycerol levels, HDL cholesterol levels, fasting glucose levels, blood pressure and medication use. The metabolic syndrome was defined according to the Adult Treatment Panel (ATP)III guidelines by the presence of three or more of: (1) waist circumference $\geq 102 \mathrm{~cm}$ for men or $\geq 88 \mathrm{~cm}$ for women; (2) serum triacylglycerol level $\geq 1.7 \mathrm{mmol} / \mathrm{L}$; (3) HDL cholesterol level $<1.03 \mathrm{mmol} / \mathrm{L}$ for men or $<1.30 \mathrm{mmol} / \mathrm{L}$ for women; (4) fasting glucose level $\geq 5.6 \mathrm{mmol} / \mathrm{L}$ or use of glucose-lowering drug medication (insulin or oral agents); or (5) systolic blood pressure $\geq 130 \mathrm{mmHg}$ and/or diastolic blood pressure $\geq 85 \mathrm{mmHg}$, and/or use of blood-pressure-lowering medication. ${ }^{24}$

\section{Sedentary behaviour variables}

Sedentary time was measured using the activPAL3 ${ }^{\text {TM }}$ physical activity monitor (PAL Technologies, Glasgow, UK). The activPAL3 is a small $(53 \times 35 \times 7 \mathrm{~mm})$, lightweight $(15 \mathrm{~g})$ triaxial accelerometer that records movement in the vertical, anteroposterior and mediolateral axes, and also determines posture (sitting or lying, standing and stepping) based on acceleration information. The device was attached directly to the skin on the front of the right thigh with transparent $3 M$ Tegaderm $^{\mathrm{TM}}$ tape, after the device had been waterproofed using a nitrile sleeve. Participants were asked to wear the accelerometer for 8 consecutive days, without removing it at any time. To avoid inaccurately identifying nonwear time, participants were asked not to replace the device once removed. Data were uploaded using the activPAL software and processed using customised software written in MATLAB R2013b (MathWorks, Natick, MA, USA). Data from the first day were excluded from the analysis because participants performed physical function tests at the research centre after the device was attached. In addition, data from the final wear day providing $\leq 14$ waking hours of data were excluded from the analysis. Participants were included if they provided at least 1 valid day ( $\geq 10 \mathrm{~h}$ of waking data).

The total amount of sedentary time was based on the sedentary posture (sitting or lying), and calculated as the mean time spent in a sedentary position during waking time per day. The method used to determine waking time has been described elsewhere. ${ }^{25}$ In brief, an automated algorithm identified wake and bed times on an individual level on multiple days, i.e. different wake and bed times for each day for each participant. The algorithm is based on the number and duration sedentary periods to identify bed times, and on the number and duration of active periods (standing or stepping) to identify wake times. The algorithm showed high accuracy in determining waking time compared with 
self-report, as the intra-class correlation coefficient (ICC) was $0.79(\mathrm{P}<0.001)$ and the mean difference in waking time between both methods was $0.02 \mathrm{~h}$ (1.2 min), with limits of agreement of -1.1 to $1.2 \mathrm{~h}$.

The number of sedentary breaks during waking time was determined as each transition from a sitting or lying position to standing or stepping, and the mean number of breaks per day was calculated. Sedentary time accumulated in a consecutive period $\geq 30$ min was defined as a prolonged sedentary bout, and the mean number of prolonged sedentary bouts during waking time per day was calculated. Average bout duration was calculated by dividing total sedentary time by the total number of sedentary bouts. Minutes with a step frequency of $>110$ steps/min were classified as higher intensity physical activity and this variable was used as a measure for moderate to vigorous physical activity (MVPA). ${ }^{26}$

\section{Covariates}

Covariates that were extracted from the questionnaire included sex, age, level of education, smoking status, alcohol consumption, mobility limitation, health status and diabetes duration. Level of education was categorised as low, medium or high, and smoking status as never, former or current smoker. Alcohol consumption was categorised as non-consumer, low consumer ( $\leq 7$ alcoholic drinks per week for women and $\leq 14$ alcoholic drinks per week for men), and high consumers ( $>7$ alcoholic drinks per week for women and >14 alcoholic drinks per week for men). Information on mobility limitation was obtained from the EuroQol-5D questionnaire and was defined as having any difficulties with walking in the previous week. Health status was determined by the presence or a history of one or more of the following conditions: cardiovascular disease, COPD, cancer or Parkinson's disease. Medication use was assessed during a medication interview and was defined as the use of glucose-lowering medication, blood-pressurelowering medication or lipid-modifying medication. Other covariates included BMI and $\mathrm{HbAlc}$, which were obtained from physical examination and laboratory assessment, as described elsewhere, ${ }^{22}$ and higher intensity physical activity.

\section{Statistical analysis}

Descriptive characteristics of the study population were summarised as mean with standard deviation (SD) or as numbers and percentages. Diabetes duration was described using the median and interquartile range. To examine differences between the groups with NGM, 
IGM and T2DM, we conducted $\chi^{2}$, ANOVA and Kruskal-Wallis tests as appropriate. General linear models were used to obtain adjusted means of the amount of sedentary time, the number of sedentary breaks, the number of prolonged sedentary bouts and the average bout duration, and these were compared between participants with NGM, IGM and T2DM. Bonferroni-corrected pairwise comparisons were made between the groups. Similar analyses were conducted to compare the adjusted means between participants without metabolic syndrome criteria, those with one or two metabolic syndrome criteria and those with the metabolic syndrome (three or more criteria). To examine associations of the sedentary behaviour variables with glucose metabolism status and the metabolic syndrome, multinomial logistic regression analyses were conducted. Results are reported as ORs with $95 \%$ confidence intervals (CI). For both general linear models and multinomial regression analyses an unadjusted model and three adjusted models were fitted. Model 1 was adjusted for sex, age, level of education and waking time. Model 2 was additionally adjusted for smoking status, alcohol consumption, health status and mobility limitation. For glucose metabolism status, model 2 was also adjusted for BMI. Model 3 was additionally adjusted for higher intensity physical activity. For the exposure variables sedentary breaks, prolonged sedentary bouts and sedentary bout duration, model 3 was also adjusted for sedentary time. The exposure variables were checked for normality, which was reasonable. Further, we tested multi-collinearity in our models: no variables had diverged confidence intervals or standard errors, or an unexpected change in the regression coefficient, and the variance-inflating factors were $<2.0$. All analyses were conducted with IBM SPSS Statistics 22.0 (IBM, Armonk, NY, USA).

\section{$\underline{\text { RESULTS }}$}

The overall study population consisted of 2,497 participants with an average age of $60.0 \pm 8.1$ years, $52 \%$ of whom were men. Table 7.1 presents descriptive characteristics in the overall study population and according to glucose metabolism status. A total of 1,395 (55.9\%) participants had NGM, 388 (15.5\%) had IGM and 714 (28.6\%) had T2DM.

Participants with T2DM were more often current smokers, were less often consumers of high levels of alcohol, had more often a mobility limitation and had a higher BMI compared with participants from the IGM and NGM groups. Those with T2DM had, on average, an $\mathrm{HbAlc}$ of $6.9 \%(51.9 \mathrm{mmol} / \mathrm{mol})$ and a median duration of diabetes of 
6 years $(Q 1-Q 3=3.0-12.0)$. Participants in all groups provided, on average, more than 6 valid days of data with an average waking time of almost $16 \mathrm{~h}$. Figure 7.1 shows the percentages of waking time spent sedentary, standing and stepping on an average day; these were statistically significantly different between the three groups $(P<0.01)$.

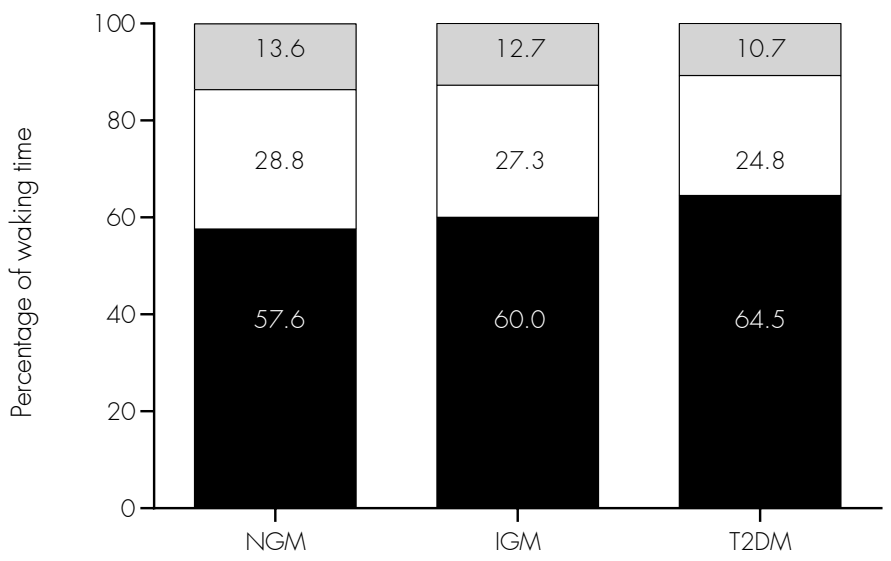

Glucose metabolism status

Figure 7.1: Percentages of waking time spent sedentary, standing and stepping according to glucose metabolism status. NGM, normal glucose metabolism; IGM, impaired glucose metabolism; T2DM, type 2 diabetes mellitus. Black bars indicate sitting/lying; white bars indicate standing; grey bars indicate stepping.

Table 7.2 presents the unadjusted and adjusted means of the sedentary behaviour variables according to glucose metabolism status. After adjustment for several confounders, a statistically significant difference of up to $26 \mathrm{~min}(0.43 \mathrm{~h})$ in sedentary time was seen between the three groups (model 3). The differences in number of sedentary breaks per day were, although statistically significantly in the unadjusted model and model 1, small between the groups in all analyses. The daily number of prolonged sedentary bouts with a duration of $30 \mathrm{~min}$ or longer differed significantly between groups in the models up to model 3. After adjustment for sedentary time and higher intensity physical activity in model 3 , the number of prolonged sedentary bouts became similar in the three groups. As was seen for the number of bouts, there were statistically significant differences in average sedentary bout duration between the groups in the models up to model 3, but after adjustment for sedentary time and higher intensity physical activity, the duration became comparable between the groups. 


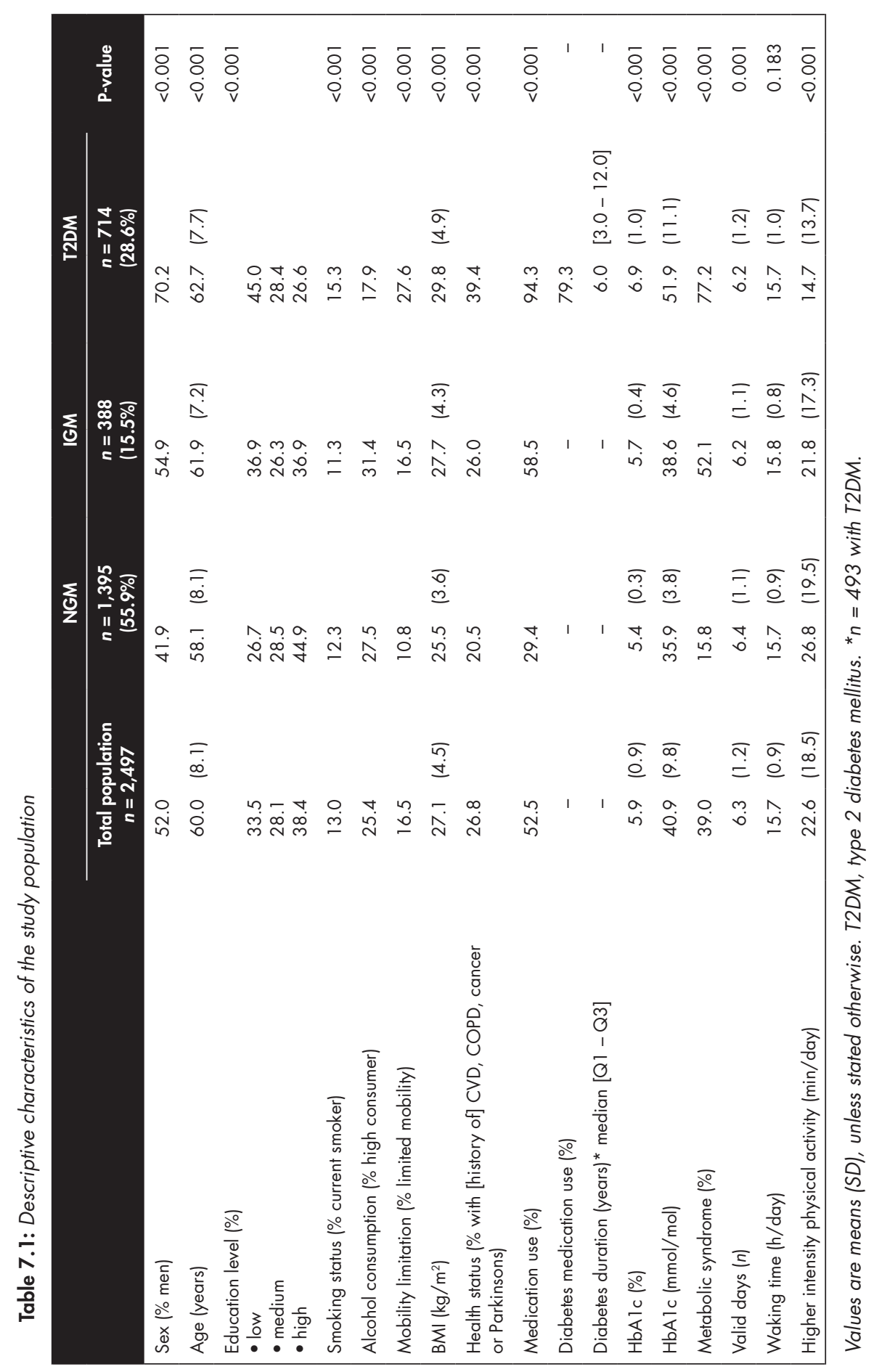




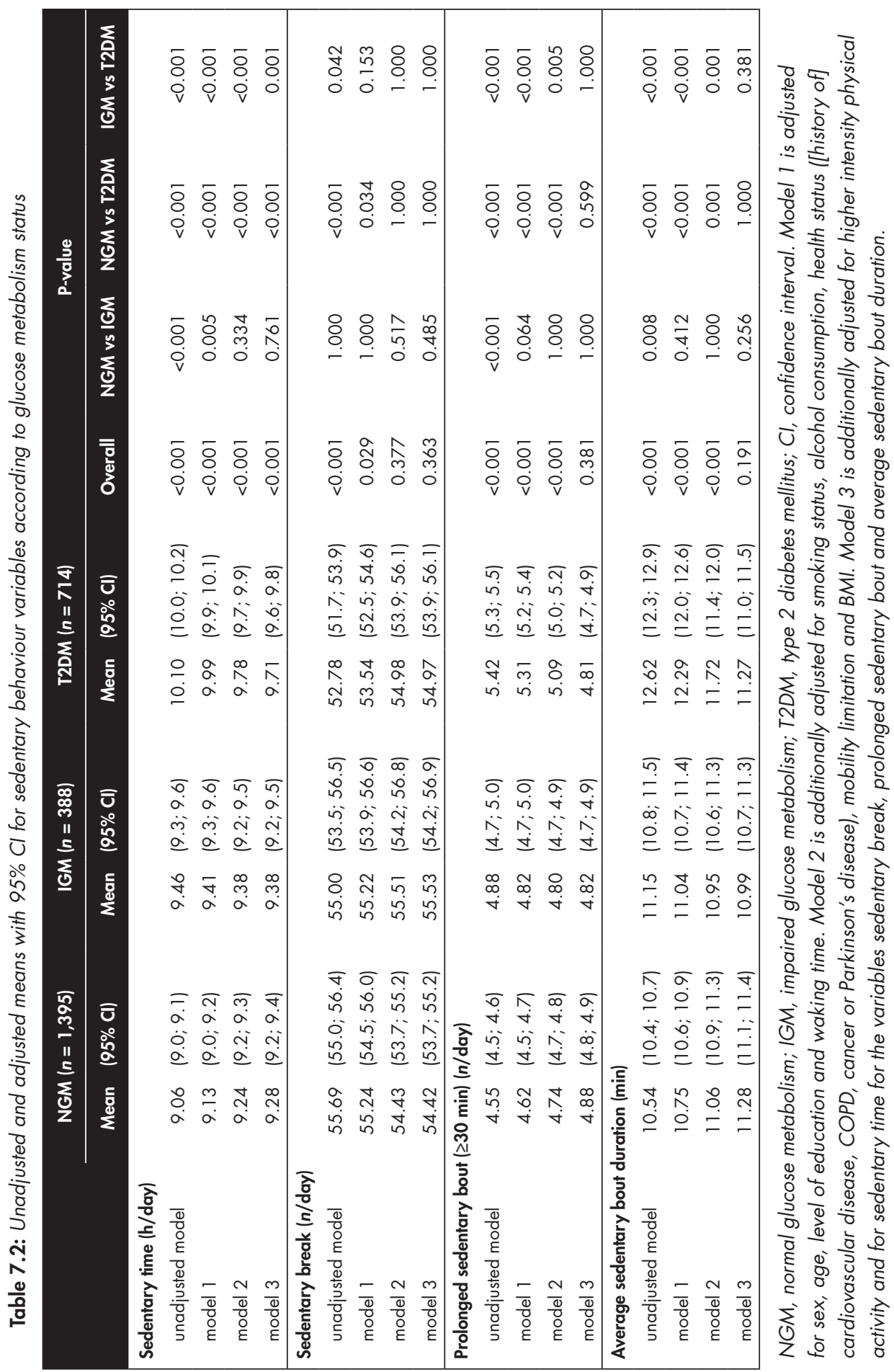


The unadjusted and adjusted means of the sedentary behaviour variables according to number of metabolic syndrome criteria are presented in Table 7.3. In all models, a gradual increase in sedentary time was seen when more metabolic syndrome criteria were present. The difference in sedentary time in model 3 was 40 min (0.66 h) per day between participants with no criteria and those with three to five criteria, and this was statistically significantly different even after adjustment for several confounders. In addition, the number of sedentary breaks per day was statistically significantly different in all models, although the difference in model 3 was only two breaks per day. The number of prolonged sedentary bouts did not differ between the groups after adjustment for confounders. The average sedentary bout duration was statistically significantly different between the groups, but the difference was less than 1 min after adjustment for relevant confounders.

In Table 7.4 the odds ratios resulting from the multinomial logistic regression analysis for glucose metabolism status and number of metabolic syndrome criteria are shown. Each additional hour of sedentary time was associated with a $1.13(95 \% \mathrm{Cl}=1.05$; $1.22)$ times higher odds for IGM and a $1.46(1.36 ; 1.56)$ times higher odds for T2DM compared with NGM (model 1). After adjustment for BMI and other covariates in models 2 and 3 , the associations remained statistically significant for T2DM $/ O R=1.22 ; 11.13$; 1.32)). The number of sedentary breaks, the number of prolonged sedentary bouts and the average sedentary bout duration were not statistically significantly associated with glucose metabolism status after adjustment for relevant confounders (model 3).

For the number of metabolic syndrome criteria, larger amounts of sedentary time were, after adjustment for several confounders, significantly associated with increased odds of $13 \%$ (1.04; $1.24)$ for having one to two criteria, and $39 \%(1.27 ; 1.53)$ for having three to five criteria compared with participants without any criteria. After adjustment for relevant confounders, the analyses of the sedentary behaviour patterns resulted in a statistically significant associations of sedentary breaks and average sedentary bout duration with three to five metabolic syndrome criteria in only model $3\left(O R_{\text {breaks }}=0.99 ;(0.98 ; 1.00) ; \mathrm{OR}_{\text {bout duration }}=1.09 ;(1.03 ; 1.15)\right)$. Additional analyses with separate adjustment for higher intensity physical activity and for sedentary time showed that for the number of prolonged sedentary bouts and average sedentary bout duration, the adjustment for sedentary time predominantly caused the change between models 2 and 3 . Additional analyses examining the number of sedentary breaks with a duration of at least $1 \mathrm{~min}$ with glucose metabolism status and the metabolic syndrome showed results similar to those for sedentary breaks of any duration; a statistically significant association was seen for three to five metabolic syndrome criteria in model 3 only $(O R=0.98 ;(0.96 ; 1.00)$ (data not shown). 
Table 7.4: Associations of sedentary behaviour variables with glucose metabolism status and number of metabolic syndrome criteria

\begin{tabular}{|c|c|c|c|c|c|c|c|c|}
\hline & \multicolumn{2}{|c|}{ Unadjusted model } & \multicolumn{2}{|c|}{ Model 1} & \multicolumn{2}{|c|}{ Model 2} & \multicolumn{2}{|c|}{ Model 3} \\
\hline & OR & $(95 \% \mathrm{Cl})$ & OR & $(95 \% \mathrm{Cl})$ & OR & $(95 \% \mathrm{Cl})$ & OR & $(95 \% \mathrm{Cl})$ \\
\hline \multicolumn{9}{|l|}{ Sedentary time (h) } \\
\hline \multicolumn{9}{|c|}{ Glucose metabolism status } \\
\hline NGM & ref & & ref & & ref & & ref & \\
\hline IGM & 1.17 & $(1.09 ; 1.25)$ & 1.13 & $(1.05 ; 1.22)$ & 1.08 & $(1.00 ; 1.17)$ & 1.07 & $(0.98 ; 1.16)$ \\
\hline T2DM & 1.49 & $(1.41 ; 1.59)$ & 1.46 & $(1.36 ; 1.56)$ & 1.28 & $(1.18 ; 1.37)$ & 1.22 & $(1.13 ; 1.32)$ \\
\hline \multicolumn{9}{|c|}{ Metabolic syndrome criteria } \\
\hline 0 criteria & ref & & ref & & ref & & ref & \\
\hline 1-2 criteria & 1.21 & $(1.12 ; 1.30)$ & 1.16 & $(1.07 ; 1.26)$ & 1.16 & $(1.07 ; 1.27)$ & 1.13 & $(1.04 ; 1.24)$ \\
\hline 3-5 criteria & 1.63 & $(1.51 ; 1.77)$ & 1.59 & $(1.46 ; 1.74)$ & 1.53 & $(1.39 ; 1.67)$ & 1.39 & $(1.27 ; 1.53)$ \\
\hline \multicolumn{9}{|c|}{ Sedentary breaks $(n)$} \\
\hline \multicolumn{9}{|c|}{ Glucose metabolism status } \\
\hline NGM & ref & & ref & & ref & & ref & \\
\hline IGM & 1.00 & $(0.99 ; 1.01)$ & 1.00 & $(0.99 ; 1.01)$ & 1.01 & $(1.00 ; 1.02)$ & 1.01 & $(1.00 ; 1.02)$ \\
\hline $\mathrm{T} 2 \mathrm{DM}$ & 0.99 & $(0.98 ; 0.99)$ & 0.99 & $(0.98 ; 1.00)$ & 1.00 & $(1.00 ; 1.01)$ & 1.01 & $(1.00 ; 1.01)$ \\
\hline \multicolumn{9}{|c|}{ Metabolic syndrome criteria } \\
\hline 0 criteria & ref & & ref & & ref & & ref & \\
\hline $1-2$ criteria & 0.99 & $(0.99 ; 1.00)$ & 1.00 & $(0.99 ; 1.01)$ & 1.00 & $(0.99 ; 1.01)$ & 1.00 & $(0.99 ; 1.01)$ \\
\hline $3-5$ criteria & 0.98 & $(0.97 ; 0.99)$ & 0.98 & $(0.97 ; 0.99)$ & 0.99 & $(0.98 ; 0.99)$ & 0.99 & $(0.98 ; 1.00)$ \\
\hline \multicolumn{9}{|c|}{ Prolonged sedentary bout ( $\geq 30 \mathrm{~min})(\mathrm{n})$} \\
\hline \multicolumn{9}{|c|}{ Glucose metabolism status } \\
\hline NGM & ref & & ref & & ref & & ref & \\
\hline IGM & 1.16 & $(1.08 ; 1.25)$ & 1.10 & $(1.02 ; 1.19)$ & 1.04 & $(0.96 ; 1.13)$ & 0.94 & $(0.83 ; 1.08)$ \\
\hline T2DM & 1.44 & $(1.36 ; 1.53)$ & 1.35 & $(1.26 ; 1.45)$ & 1.17 & $(1.09 ; 1.26)$ & 0.91 & $(0.81 ; 1.03)$ \\
\hline \multicolumn{9}{|c|}{ Metabolic syndrome criteria } \\
\hline 0 criteria & ref & & ref & & ref & & ref & \\
\hline $1-2$ criteria & 1.21 & $(1.11 ; 1.31)$ & 1.15 & $(1.05 ; 1.25)$ & 1.15 & $(1.05 ; 1.25)$ & 1.01 & $(0.88 ; 1.17)$ \\
\hline 3-5 criteria & 1.62 & $(1.48 ; 1.76)$ & 1.51 & $(1.38 ; 1.66)$ & 1.45 & $(1.32 ; 1.60)$ & 1.07 & $(0.91 ; 1.24)$ \\
\hline \multicolumn{9}{|c|}{ Average sedentary bout duration (min) } \\
\hline \multicolumn{9}{|c|}{ Glucose metabolism status } \\
\hline NGM & ref & & ref & & ref & & ref & \\
\hline IGM & 1.06 & $(1.02 ; 1.10)$ & 1.03 & $(1.00 ; 1.07)$ & 0.99 & $(0.96 ; 1.03)$ & 0.96 & $(0.92 ; 1.01)$ \\
\hline $\mathrm{T} 2 \mathrm{DM}$ & 1.17 & $(1.14 ; 1.21)$ & 1.13 & $(1.10 ; 1.16)$ & 1.05 & $(1.02 ; 1.08)$ & 0.99 & $(0.95 ; 1.02)$ \\
\hline \multicolumn{9}{|c|}{ Metabolic syndrome criteria } \\
\hline 0 criteria & ref & & ref & & ref & & ref & \\
\hline 1-2 criteria & 1.11 & $(1.06 ; 1.15)$ & 1.07 & (1.03; 1.12) & 1.07 & $(1.02 ; 1.12)$ & 1.03 & $(0.98 ; 1.08)$ \\
\hline 3-5 criteria & 1.27 & $(1.22 ; 1.32)$ & 1.21 & $(1.16 ; 1.27)$ & 1.19 & $(1.14 ; 1.25)$ & 1.09 & $(1.03 ; 1.15)$ \\
\hline
\end{tabular}

Regression results are presented as odds ratios (OR) with $95 \%$ confidence intervals (95\% Cl). Boldface indicates statistical significance ( $P<0.05)$. NGM, normal glucose metabolism; IGM, impaired glucose metabolism; T2DM, type 2 diabetes mellitus. Model 1 is adjusted for sex, age, level of education and waking time. Model 2 is additionally adjusted for smoking status, alcohol consumption, health status ([history of] cardiovascular disease, COPD, cancer or Parkinson's disease) and mobility limitation. Glucose metabolism status is also additionally adjusted for BMI. Model 3 is additionally adjusted for higher intensity physical activity and for sedentary time for the variables sedentary break, prolonged sedentary bout and average sedentary bout duration. 


\section{DISCUSSION}

To our knowledge, our study is the largest in which posture-discriminating accelerometry was used to objectively measure total amount and patterns of sedentary behaviour in a sample of adults comprising participants with T2DM, IGM or NGM. The results show that participants with T2DM had the most sedentary time, up to 26 min more per day compared with participants with IGM or NGM. Each extra hour of sedentary time was associated with increased odds for T2DM of $22 \%$. No statistically significant differences were observed between participants with NGM and those with IGM. More time spent sedentary was also associated with a 1.13 times higher odds for one to two metabolic syndrome criteria and a 1.39 times higher odds for the metabolic syndrome (three to five criteria), independent of higher intensity physical activity. The number of sedentary breaks per day was highly comparable and not significantly different between the participants with T2DM, those with IGM and those with NGM. A statistically significant difference in the number of breaks was seen between participants with the metabolic syndrome and those without the metabolic syndrome, but the difference was only two breaks per day. Also, the odds ratio for the metabolic syndrome was statistically significant but small, and therefore not clinically relevant. Similar results were found for average sedentary bout duration: a statistically significant difference in bout duration was seen between participants with the metabolic syndrome and those without the metabolic syndrome, but the difference was less than $1 \mathrm{~min}$. Also, the OR for the metabolic syndrome was statistically significant: a 1.09 times higher odds for three to five criteria when the average sedentary bout was longer. Last, the number of prolonged sedentary bouts did not differ between the groups according to diabetes status or the groups according to the metabolic syndrome, and the odds ratios were not statistically significant.

A major strength of our study was the use of posture-based measurement with the activPAL accelerometer, worn on the thigh, which has shown to be a highly accurate method for assessing sedentary behaviour. ${ }^{20,21}$ Thus, our estimates of the amounts of sedentary time and the determination of sedentary breaks are more accurate than data based solely on acceleration, which cannot discriminate between postures. Further, we used accelerometry in a large sample of middle-aged and older adults with T2DM and IGM, which enabled us to examine and quantify associations of several objectively measured sedentary behaviour variables with T2DM. Also, waterproofed attachment of the activPAL on the thigh enabled us to collect $24 \mathrm{~h}$ accelerometry data, which not 
only resulted in complete data assessments, but could also have improved wear-time compliance, as demonstrated in a recently published study in children. ${ }^{27}$ Wear time in our study population was, on average, 6.3 days with $15.7 \mathrm{~h}$ of waking time, and $85.3 \%$ of our study population provided at least 6 valid days of data. Another strength was adjustment for important confounders including BMl and higher intensity physical activity, which excludes the possibility that these factors account for the associations of sedentary behaviour with T2DM and the metabolic syndrome. The adjustment for BMI predominantly caused the differences between models 1 and 2, yet BMl could be part of the pathway between sedentary behaviour and T2DM. Consequently, the analyses could have been subject to overadjustment.

A few limitations should also be mentioned, of which study design is the most important. As our analyses were cross-sectional in nature, causal relationships could not be examined. It may therefore be possible that participants with T2DM had more sedentary time because of their poorer health. However, when participants with T2DM on insulin medication (who may be considered to have more severe T2DM and could for that reason have more sedentary time) were excluded from the analysis, the results did not change. This may suggest that sedentary behaviour is at least partly preceded T2DM, as the associations were similar among participants who did not necessarily have to spend more time sedentary because of their health (data not shown). Furthermore, previous prospective studies have demonstrated that sedentary behaviour predicts markers of insulin resistance. ${ }^{5,13}$ Taken together, these findings support the hypothesis that the direction of the association is predominantly from sedentary behaviour to health outcomes, although large-scale prospective studies are warranted to provide better insights into the directions of the associations. Further, although we adjusted for a broad range of confounding factors, it is possible that some unmeasured factors, for example dietary intake, partly explain the associations. Finally, sedentary behaviour was measured during 1 week only, and this may not truly reflect habitual behaviour.

Several previous studies have used accelerometry to objectively measure sedentary behaviour and examined its associations with the metabolic syndrome. In line with our results, larger amounts of sedentary time have been associated with metabolic risk, although the reported effect sizes were smaller..$^{8,11,15-17}$ To date, no studies have reported associations between objectively measured sedentary time as exposure variable and T2DM as outcome measure. However, a meta-analysis of studies with self-reporting measures of sedentary behaviour showed a risk of $112 \%$ for T2DM in the group with 
the largest compared with the smallest amounts of sedentary time. ${ }^{28}$ Furthermore, large amounts of objectively measured sedentary time have been associated with (markers of) insulin resistance $5,6,10$ and, as mentioned earlier, the metabolic syndrome. As these factors are precursors to T2DM, the results of these studies in combination with the results of the meta-analysis may support our findings of an increased risk for T2DM with increasing amount of sedentary time. Physiological mechanisms that could explain our findings have not yet been studied extensively, but results from animal studies suggest that responses to contractile (in)activity of muscle cells can play a role in glucose metabolism as reductions in lipoprotein lipase (LPL), an enzyme that contributes to the metabolism and transport of lipids, were seen after periods of inactivity. ${ }^{29,30}$

Few observational studies have reported associations between patterns of sedentary behaviour and metabolic health, but the findings that have been reported are inconsistent. Some studies have shown associations between the number of sedentary breaks and metabolic risk factors ${ }^{6,15,18}$, while others have not. ${ }^{5,16}$ These inconsistencies could be caused by different methods for measuring breaks, which can be based on change in acceleration or on change in posture. Also, differences exist in the definition of a break, which can be any interruption of sedentary time or interruptions of at least $1 \mathrm{~min}$, although we did not find different results for sedentary breaks of any duration and breaks of at least $1 \mathrm{~min}$. Further, comparability of studies is hampered by differences in adjustment strategies and, furthermore, associations could be different among younger and older adults and among adults with and without (a higher risk for) T2DM, because their metabolic profiles differ. Studies on prolonged sedentary bouts and sedentary bout duration are scarce and no study on associations with T2DM has been reported. However, the studies of Bankoski ${ }^{15}$ and Healy ${ }^{7}$ show no statistically significant associations of bout length or number of bouts $\geq 30$ min with metabolic variables, except waist circumference. Further, in our study we used three measures for expressing the sedentary behaviour pattern, but other measures could also be used to study the pattern of sedentary time. ${ }^{31}$ In order to compare studies examining associations of numbers of both sedentary breaks and bouts, future studies should use similar measures, and ideally adjust for similar confounders.

To conclude, this was the largest study that objectively measured total amount and patterns of sedentary behaviour in a sample of adults with T2DM, IGM or NGM. The results showed that an extra hour of sedentary time was associated with increased odds of $22 \%$ for T2DM and of $39 \%$ for the metabolic syndrome, independent of higher intensity physical activity. The pattern in which sedentary time was accumulated, as expressed 
by number of sedentary breaks, number of prolonged sedentary bouts and average sedentary bout duration, was only weakly associated with an increased risk for the metabolic syndrome. Future studies in participants with T2DM should be conducted to confirm our results, and to explore dose-response relationships and causality. Nevertheless, our findings could have important implications for public health as they suggest that sedentary behaviour may play a significant role in the development and prevention of T2DM, independent of higher intensity physical activity. Consideration should be given to including strategies to reduce the amount of sedentary time in diabetes prevention programmes. 


\section{REFERENCES}

1. Wild S, Roglic G, Green A, Sicree R, King H. Estimates for the year 2000 and projections for 2030. Diabetes Care. 2004;27(5):1047-53.

2. American Diabetes Association. Standards of medical care in diabetes-2014. Diabetes Care. 2014.

3. International Diabetes Federation. Global guideline for type 2 diabetes. 2012.

4. Sedentary Behaviour Research Network. Standardized use of the terms "sedentary" and "sedentary behaviours." Appl Physiol Nutr Metab. 2012;37:540-2.

5. Cooper AR, Sebire S, Montgomery AA, Peters TJ, Sharp DJ, Jackson N, et al. Sedentary time, breaks in sedentary time and metabolic variables in people with newly diagnosed type 2 diabetes. Diabetologia. 2012;55(3):589-99.

6. Healy GN, Matthews CE, Dunstan DW, Winkler EAH, Owen N. Sedentary time and cardiometabolic biomarkers in US adults: NHANES 2003-06. Eur Heart J. 2011 ;32(5):590-7.

7. Healy $\mathrm{GN}$, Winkler $\mathrm{EAH}$, Brakenridge $\mathrm{CL}$, Reeves MM, Eakin EG. Accelerometer-derived sedentary and physical activity time in overweight/obese adults with type 2 diabetes: Cross-sectional associations with cardiometabolic biomarkers. PLoS One. 2015;10:e0119140.

8. Healy G, Wijndaele K, Dunstan D, Shaw J, Salmon J, Zimmet $P$, et al. Objectively measured sedentary time, physical activity, and metabolic risk. Diabetes Care. 2008;31 (2):369-71.

9. Gennuso KP, Gangnon RE, Matthews CE, ThraenBorowski KM, Colbert LH. Sedentary behavior, physical activity, and markers of health in older adults. Med Sci Sports Exerc. 2013;45(8):1493500.

10. Brocklebank LA, Falconer CL, Page AS, Perry R, Cooper AR. Accelerometer-measured sedentary time and cardiometabolic biomarkers: A systematic review. Prev Med. 2015;76:92-102.

11. Henson J, Yates T, Biddle SJH, Edwardson CL, Khunti K, Wilmot EG, et al. Associations of objectively measured sedentary behaviour and physical activity with markers of cardiometabolic health. Diabetologia. 2013;56:1012-20.
12. Lahjibi E, Heude B, Dekker JM, Højlund K, Laville $M$, Nolan J, et al. Impact of objectively measured sedentary behaviour on changes in insulin resistance and secretion over 3years in the RISC study: Interaction with weight gain. Diabetes Metab. 2013; 39(3):217-25.

13. Wiindaele K, Orrow G, Ekelund U, Sharp SJ, Brage S, Griffin SJ, et al. Increasing objectively measured sedentary time increases clustered cardiometabolic risk: A 6 year analysis of the ProActive study. Diabetologia. 2014;57(2):30512.

14. Barone Gibbs B, Pettee Gabriel K, Reis JP, Jakicic JM, Carnethon MR, Sternfeld B. Cross-sectional and longitudinal associations between objectively measured sedentary time and metabolic disease: The Coronary Artery Risk Development in Young Adults (CARDIA) Study. Diabetes Care. 2015 Oct;38(10): 1835-43

15. Bankoski A, Harris TB, McClain JJ, Brychta RJ, Caserotti P, Chen KY, et al. Sedentary activity associated with metabolic syndrome independent of physical activity. Diabetes Care. $2011 ; 34(2): 497-503$.

16. Gennuso KP, Gangnon RE, Thraen-Borowski KM, Colbert LH. Dose-response relationships between sedentary behaviour and the metabolic syndrome and its components. Diabetologia. 2014;58:485-92.

17. Cooper AMM, Brage S, Ekelund U, Wareham NJ, Griffin SJ, Simmons RK. Association between objectively assessed sedentary time and physical activity with metabolic risk factors among people with recently diagnosed type 2 diabetes. Diabetologia. 2014;57(1):73-82.

18. Healy G, Dunstan D, Salmon J, Cerin E, Shaw $\mathrm{J}$, Zimmet $P$, et al. Breaks in sedentary time. Diabetes Care. 2008;31(4):661-3.

19. Benatti F, Ried-Larsen M. The effects of breaking up prolonged sitting time. Med Sci Sport Exerc. 2015;47(10):2053-61.

20. Godfrey A, Culhane KM, Lyons GM. Comparison of the performance of the activPAL ${ }^{T M}$ Professional physical activity logger to a discrete accelerometer-based activity monitor. Med Eng Phys. 2007;29(8):930-4.

21. Kozey-Keadle S, Libertine A, Lyden K, Staudenmayer J, Freedson PS. Validation of wearable monitors for assessing 
sedentary behavior. Med Sci Sport Exerc. $2011 ; 43(8): 1561-7$.

22. Schram MT, Sep SJS, van der Kallen CJ, Dagnelie PC, Koster A, Schaper N, et al. The Maastricht Study: An extensive phenotyping study on determinants of type 2 diabetes, its complications and its comorbidities. Eur J Epidemiol. 2014;29(6):439-51.

23. World Health Organization. Definition and Diagnosis of Diabetes Mellitus and Intermediate Hyperglycemia. World Health Organization. 2006.

24. Alberti KGMM, Eckel RH, Grundy SM, Zimmet $P Z$, Cleeman JI, Donato K a., et al. Harmonizing the metabolic syndrome. Circulation. 2009; 120(16): 1640-5.

25. Van der Berg J, Willems P, Van der Velde J, Savelberg H, Schaper N, Schram M, et al. Identifying waking time in 24-h accelerometry data in adults using an automated algorithm. J Sports Sci. 2016;2:1-7.

26. Tudor-Locke C, Rowe DA. Using cadence to study free-living ambulatory behaviour. Sports Med. 2012;42(5):381-98.

27. Tudor-Locke C, Barreira TV, Schuna JM, Mire $E F$, Chaput J-P, Fogelholm M, et al. Improving wear time compliance with a 24-hour waistworn accelerometer protocol in the International Study of Childhood Obesity, Lifestyle and the Environment (ISCOLE). Int J Behav Nutr Phys Act. $2015 ; 12(11): 11$.

28. Wilmot EG, Edwardson CL, Achana FA, Davies M, Gorely T, Gray L, et al. Sedentary time in adults and the association with diabetes, cardiovascular disease and death: Systematic review and meta-analysis. Diabetologia. 2012;55(11):2895-905.

29. Hamilton MT, Hamilton DG, Zderic TW. Sedentary Behavior as a mediator of type 2 diabetes. In: Diabetes and Physical Activity. 2014. p. 11-26.

30. Hamilton M, Hamilton D, Zderic T. Role of low energy expenditure and sitting in obesity, metabolic syndrome, type 2 diabetes, and cardiovascular disease. Diabetes. 2007; 56:2655-67.

31. Chastin SFM, Granat MH. Methods for objective measure, quantification and analysis of sedentary behaviour and inactivity. Gait Posture. 2010;31(1):82-6. 
Chapt

照 


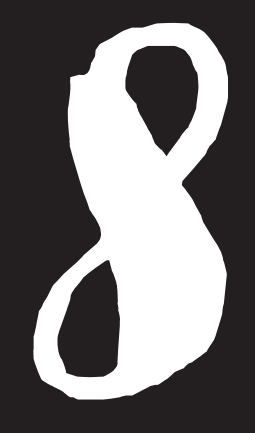

Associations of total amount and patterns of physical activity and sedentary behaviour with aortic pulse wave velocity: The Maastricht Study

JULIANNE D. VAN DER BERG | ANNEMARIE KOSTER | HANS BOSMA | VERONICA G. ONETE | HANS H.C.M. SAVELBERG | KOEN D. REESINK | MIRANDA T. SCHRAM | SIMONE J.S. SEP | CARLA J.H. VAN DER KALLEN | PIETER C. DAGNELIE | NICOLAAS C. SCHAPER | RONALD M.A. HENRY | COEN D.A. STEHOUWER 


\section{A B STRACT}

Background: Since aortic stiffness has been related to cardiovascular morbidity and mortality, it is relevant to examine what modifiable factors are associated with this phenomenon. Therefore, we examined cross-sectional associations of total amount and patterns of physical activity and sedentary behaviour with aortic pulse wave velocity (carotid-femoral pulse wave velocity (cfPWV)).

Methods: We included 2,029 participants (52.1\% men, age (mean $\pm S D$ ): $60.2 \pm 8.0$ years) of The Maastricht Study who were asked to wear an activPAL3 accelerometer $24 \mathrm{~h} /$ day on 8 consecutive days. We calculated daily amounts of total physical activity (TPA), higher intensity physical activity (HPA, i.e. $>110$ steps/min), lower intensity physical activity (LPA), and sedentary time; daily numbers of sedentary breaks and prolonged sedentary bouts ( $\geq 30 \mathrm{~min}$ ); and average sedentary bout duration. CFPWV was determined using applanation tonometry.

Results: On average $60 \%$ of waking time $(9.4 \pm 1.7 \mathrm{~h} /$ day $)$ was sedentary behaviour and $13 \%$ was TPA. After adjustment for potential confounders, each extra hour of TPA and each extra 30 minutes of HPA were associated with a lower cfPWV $\left(B_{\text {TPA }}=0.99\right.$; $B_{\text {HPA }}=0.98$; both $\mathrm{P}<0.05$; back-transformed from log-scale). LPA, sedentary time, sedentary breaks, prolonged sedentary bouts, and average sedentary bout duration were not statistically significantly associated with cPPW affer adjustment potential confounders.

Conclusions: This study is the first in which total amount and patterns of objectively measured daily activity were examined in relation to cFPV. Larger amounts of TPA and HPA were associated with lower cfPWV whereas no associations of LPA and (patterns of) sedentary behaviour with cFWV were observed. 


\section{INTRODUCTION}

Aortic stiffness, which can be assessed by measuring aortic pulse wave velocity (aPWV), has been shown to be related to cardiovascular events and mortality. ${ }^{1,2}$ Ageing and common conditions such as hypertension and type 2 diabetes mellitus are major risk factors for aortic stiffness, but also modifiable lifestyle factors have been associated with stiffening of the arterial vessels. ${ }^{3-6}$

High intensity physical activity (>6 metabolic equivalents $(M E T s)^{7}$ ) is one of the factors that have been broadly studied in relation to aortic stiffness. A recently published meta-analysis has reported that aerobic exercise interventions improved measures of aPWV significantly. ${ }^{6}$ However, only a (very) small part of all daily activity is high intensity physical activity. Most physical activity such as walking and cleaning is executed at a lower intensity level (<3 METs).,8 Whether this habitual physical activity is also favourably associated with aPWV is not clear as studies have shown inconsistent results. ${ }^{9-13}$ Further, most time of the day is spent being sedentary. ${ }^{14}$ Sedentary behaviour is defined as any waking behaviour that is characterised by a low energy expenditure (<1.5 METs) such as watching TV, using the computer or driving. ${ }^{15}$ Large amounts of sedentary time have been associated with cardiovascular risk factors, ${ }^{16,17}$ and sedentary behaviour is therefore another lifestyle factor which should be studied next to physical activity.

Accelerometers can be used for the objective measurement of physical activity and sedentary behaviour. These devices record acceleration of the body segment to which the device is attached, and provide accurate information about frequency, duration and intensity of activity. ${ }^{18}$ To date, only a few observational studies have used accelerometry data to examine associations of physical activity and sedentary behaviour with aPWV. The results were inconsistent as some researchers found associations of physical activity ${ }^{9-12}$ and/or sedentary behaviour with aPWV, $10,12,13$ while others did not. $9,11,13$ Further, in these studies sedentary behaviour was most often expressed as the total amount of sedentary time, although the pattern in which sedentary time is accumulated may also be relevant. Sedentary behaviour patterns can be expressed by sedentary breaks, which refers to interruptions of sedentary time, or sedentary bouts, which refers to sedentary periods of a certain duration without interruptions. Although these patterns have been studied in relation to cardiovascular risk factors ${ }^{17,19-22}$ and parameters of arterial stiffness, ${ }^{23}$ no studies have been published on aPWV. 
In view of the above, it is important to examine associations of physical activity and sedentary behaviour with aortic stiffness. Therefore, we measured total amount and patterns of habitual daily activity with the thigh-worn activPAL3 ${ }^{\mathrm{TM}}$ accelerometer. This device classifies activity using data on acceleration combined with posture, which has shown to be an accurate method. ${ }^{24,25}$ The aim of this study was to examine cross-sectional associations of the total amount and patterns of physical activity and sedentary behaviour with aPWV, in a large sample of adults aged 40-75 years who participated in The Maastricht Study.

\section{METHODS}

\section{Study population}

We used data from The Maastricht Study, an observational prospective population-based cohort study. The rationale and methodology have been described previously. ${ }^{26}$ In brief, the study focuses on the aetiology, pathophysiology, complications and comorbidities of type 2 diabetes mellitus (T2DM) and is characterized by an extensive phenotyping approach. Eligible participants were individuals aged between 40 and 75 years and living in the southern part of the Netherlands. Participants were recruited through mass media campaigns and from the municipal registries and the regional Diabetes Patient Registry via mailings. Recruitment was stratified according to known type 2 diabetes status, with an oversampling of individuals with T2DM, for reasons of efficiency. This study included cross-sectional data from the first 3,451 participants, who completed the baseline survey between November 2010 and September 2013. After excluding participants who did not receive an accelerometer due to logistics $(n=673)$, whose accelerometer measurement failed $(n=136)$, who had no vascular measurements data due to logistics or a failed measurement ( $n=479)$, or who had other missing data ( $n=134)$, a total of 2,029 participants were included in the present analyses.

The study has been approved by the institutional medical ethical committee (NL3 1329.068.10) and the Minister of Health, Welfare and Sports of the Netherlands (Permit 131088 -105234-PG). All participants gave written informed consent. 


\section{Measurements}

\section{Carotid-femoral pulse wave velocity}

The measurements were performed by trained vascular technicians, in a dark, quiet temperature controlled room $\left(21-23^{\circ} \mathrm{C}\right)$. Participants were asked to refrain from smoking and drinking coffee or tea or alcoholic beverages three hours prior to the study. Participants were allowed to have a light meal (breakfast and/or lunch). All measurements were performed in the supine position after 10 min of rest. Talking or sleeping was not allowed during the examination. During the measurements (approximately $15 \mathrm{~min}$ ), heart rate and mean arterial pressure were determined every $5 \mathrm{~min}$ with an oscillometric device (Accutorr Plus, Datascope, Montvale, NJ, USA). A three-lead electrocardiogram was recorded continuously during the measurements to facilitate automatic signal processing.

Carotid to femoral pulse wave velocity (cFPWV) was determined according to recent guidelines ${ }^{27}$ with the use of applanation tonometry (SphygmoCor, Atcor Medical, Sydney, Australia). Pressure waveforms were determined at the right common carotid and right common femoral arteries. Difference in the time of pulse arrival from the R-wave of the electrocardiogram between the two sites (transit time) was determined with the intersecting tangents algorithm. The pulse wave travel distance was calculated as $80 \%$ of the direct straight distance (measured with an infantometer) between the two arterial sites. The median of three cfPWV (defined as traveled distance/transit time) recordings was used in the analyses. Reproducibility was assessed in 12 individuals 16 men; $60.8 \pm 6.8$ years; 6 T2DM) who were examined by two observers at two occasions spaced one week apart. The intra- and inter-observer intra-class correlation coefficients were 0.87 and 0.69 . CfPWV was transformed using its natural logarithm, because of a skewed distribution.

\section{Accelerometry data}

Daily activity levels and patterns were measured using the activPAL3 ${ }^{\mathrm{TM}}$ physical activity monitor (PAL Technologies, Glasgow, UK). The activPAL3 ${ }^{\text {TM }}$ is a small $(53 \times 35 \times 7 \mathrm{~mm}$ $\mathrm{mm}$ ), lightweight (15 gram) triaxial accelerometer that records movement in the vertical, anteroposterior and mediolateral axes, and also determines posture (sitting or lying, standing, and stepping) based on acceleration information. The device was attached directly to the skin on the front of the right thigh with transparent $3 M$ Tegaderm $^{\text {TM }}$ tape, after the device had been waterproofed using a nitrile sleeve. Participants were asked to wear the accelerometer for 8 consecutive days, without removing the device at any time. To avoid inaccurately identifying of non-wear time, participants were asked not to replace 
the device once removed. Data were uploaded using the activPAL software and processed using customised software written in MATLAB R2013b (MathWorks. Natick, MA, USA). Data from the first day were excluded from the analysis because participants performed physical function tests at the research centre after the device was attached. In addition, data from the final wear day providing $\leq 14$ waking hours of data were excluded from the analysis. Participants were included if they provided at least 1 valid day $(\geq 10 \mathrm{~h}$ of waking data).

The total amount of physical activity was based on the stepping posture and calculated as the mean time spent stepping during waking time per day. The method used to determine waking time has been described elsewhere. ${ }^{28}$ Physical activity (stepping time) was further classified into higher intensity physical activity (HPA; minutes with a step frequency $>110$ steps/min during waking time $)^{29,30}$ and lower intensity physical activity (LPA; minutes with a step frequency $\leq 110$ steps/min during waking time. Standing time was not included.). The total amount of sedentary time was based on the sedentary posture (sitting or lying), and calculated as the mean time spent in a sedentary position during waking time per day. The number of sedentary breaks during waking time was determined as each transition from a sitting or lying position to standing or stepping with a duration of at least $1 \mathrm{~min}$, and the mean number of breaks per day was calculated. Sedentary time accumulated in a consecutive period $\geq 30 \mathrm{~min}$ was defined as a prolonged sedentary bout, and the mean number of prolonged sedentary bouts during waking time per day was calculated. Average bout duration was calculated by dividing total sedentary time by total number of sedentary bouts of any duration.

\section{Covariates}

Covariates which were extracted from questionnaire included sex, age, level of education, smoking status, alcohol consumption, mobility limitation, and cardiovascular disease. Level of education was categorized into low, medium and high, and smoking status into never, former and current smoker. Alcohol consumption was categorized into non-consumers, low consumers ( $\leq 7$ glasses per week for women and $\leq 14$ glasses per week for men), and high consumers (>7 glasses per week for women and >14 glasses per week for men). Mobility limitation was obtained from the EuroQol-5D questionnaire and was defined as having any difficulties with walking in the previous week. Cardiovascular disease was defined as a self-reported history of myocardial infarction, cerebrovascular infarction or haemorrhage, or percutaneous artery angioplasty of, or vascular surgery on, the coronary, abdominal, 
peripheral or carotid arteries. Medication use was assessed during a medication interview and was defined as the use of antihypertensive medication or lipid-modifying medication. Other covariates included body mass index (BMI), total and HDL cholesterol, triglycerides, and glucose metabolism status (normal, impaired, T2DM) which were obtained from physical examination and laboratory assessment as described elsewhere. ${ }^{26}$ Lastly, mean arterial pressure and heart rate as assessed during the vascular measurement were included as covariates.

\section{Statistical analysis}

Descriptive characteristics of the study population were summarized as means with standard deviations (SD) or as numbers and percentages. Non-normally distributed variables were described using the median and the interquartile range. Chi-square, ANOVA and Mann-Whitney tests were conducted as appropriate to compare participants with a cfPWV below the median and equal to or above the median (cFPWV $=8.65 \mathrm{~m} / \mathrm{s}$ ). The outcome measure, cfPWV, had a skewed distribution and was therefore log-transformed. General linear models were used to obtain means of cfPWV in tertiles of total physical activity and sedentary behaviour. Linear regression analysis was used to examine whether the three physical activity and the four sedentary behaviour variables were associated with cfPWV. In models 1 the associations were adjusted for sex, age, waking time and glucose metabolism status (to take into account the oversampling of participants with T2DM in The Maastricht Study). In models 2 the associations were additionally adjusted for mean arterial pressure, heart rate, level of education, smoking status, alcohol consumption, $\mathrm{BMI}$, total-to-HDL cholesterol ratio, triglycerides, history of cardiovascular disease, use of anti-hypertensive medication, use of lipid-modifying medication, and mobility limitation. In models 3 the association with HPA was additionally adjusted for sedentary time; the association with sedentary time was additionally adjusted for HPA; and the associations with sedentary breaks, prolonged sedentary bouts, and average sedentary bout duration were additionally adjusted for HPA and sedentary time. Results are reported as unstandardized coefficients (B), back-transformed from the natural log scale. All analyses were conducted with IBM SPSS Statistics 22.0 (IBM Corp. Armonk, NY, USA). 


\section{$\underline{\text { RESULTS }}$}

Table 8.1 presents descriptive characteristics of the study population and in the groups according to cfPWV. Of the total population $52.1 \%$ was man and the average age was $60.2 \pm 8.0$ years. The accelerometer was worn on average 6 days, but almost $56 \%$ of the participants provided 7 valid days of data. Average waking time was $15.7 \pm 0.9 \mathrm{~h}$ per day, of which most time was spent sedentary $(60 \%)$ and only $27 \%$ respectively $13 \%$ was spent standing and being physically active (stepping time). Of the total amount of physical activity, most time (82\%) was LPA and only $18 \%$ was HPA ( $2 \%$ of total waking time). The median of the average sedentary bout duration was $11 \mathrm{~min}$ and the number of long sedentary bouts (accumulated in a consecutive period $\geq 30 \mathrm{~min}$ ) was on average 5 per day. Sedentary time was interrupted on average 55 times per day (sedentary breaks). The physical activity and sedentary behaviour variables were all statistically significantly different between participants with a cfPWV below the median and participants with a cfPWV equal to or above the median. Participants with a cfPWV below the median had more HPA, spent less time sedentary, and had more sedentary breaks, fewer prolonged sedentary bouts and a shorter average sedentary bout duration compared to those with a cfPWV equal to or above the median. 


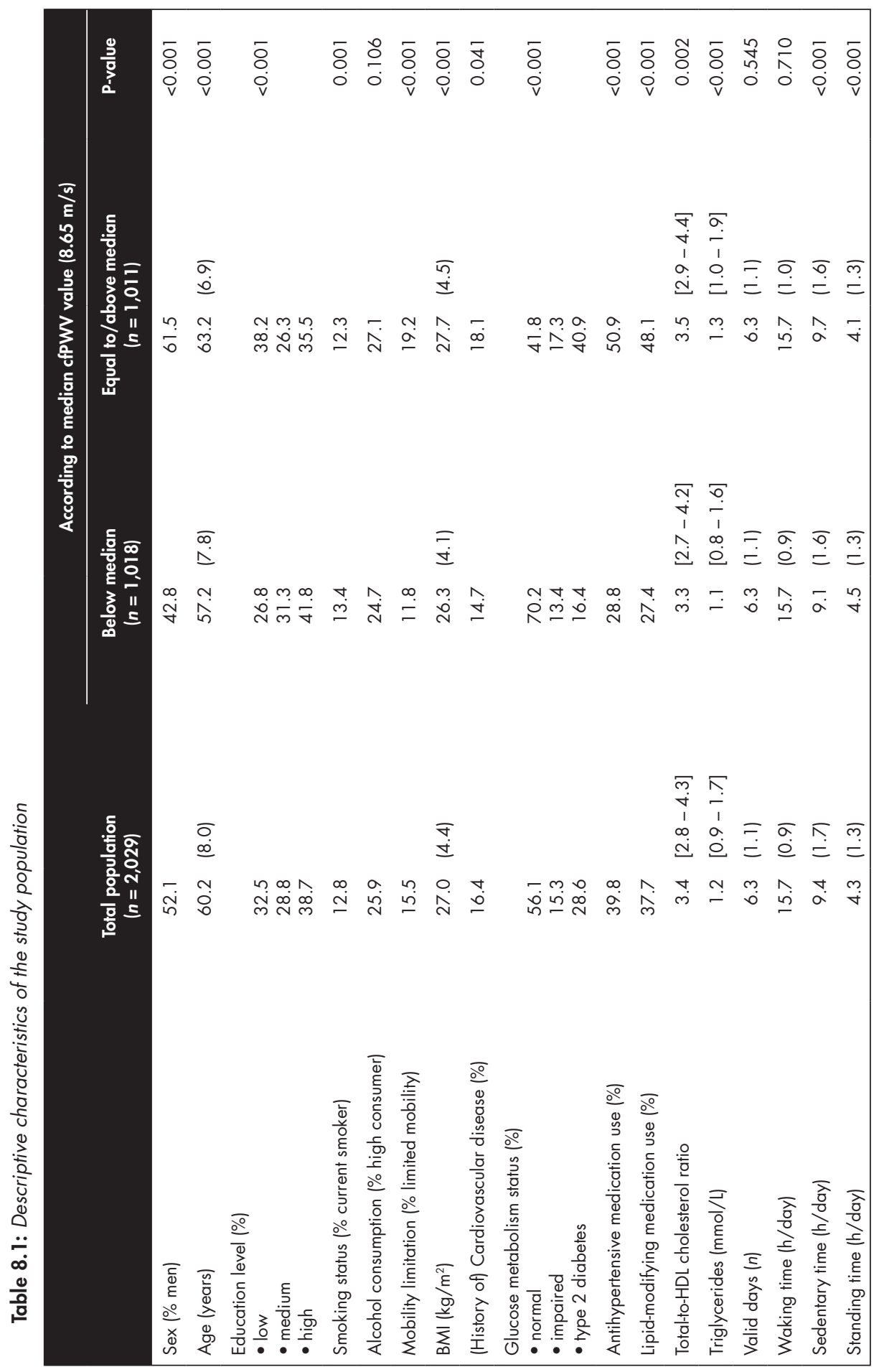




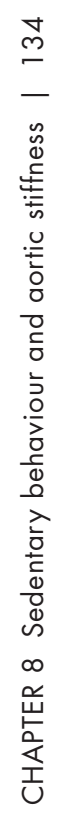

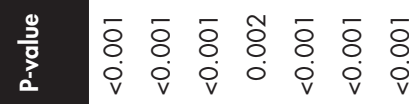

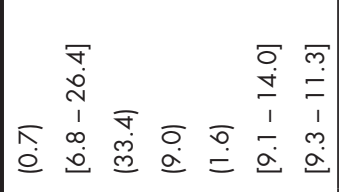

ㅎํำ

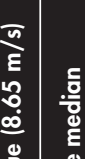

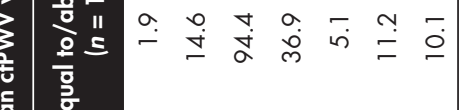

홍 훙

웡

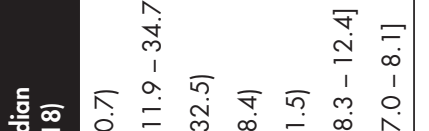

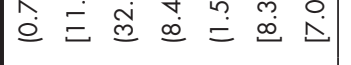

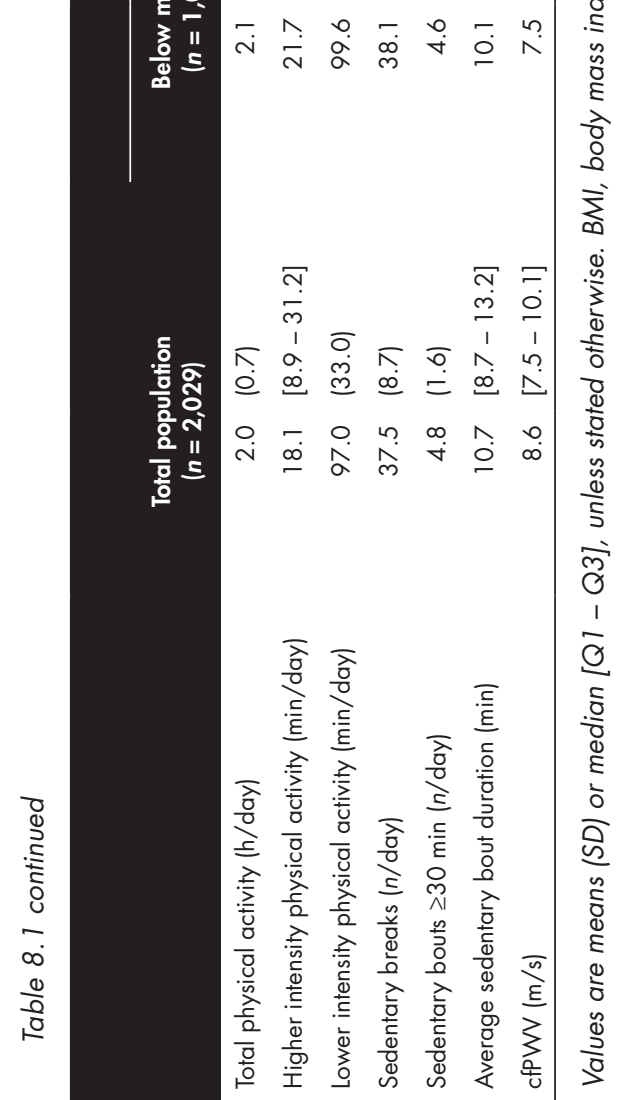

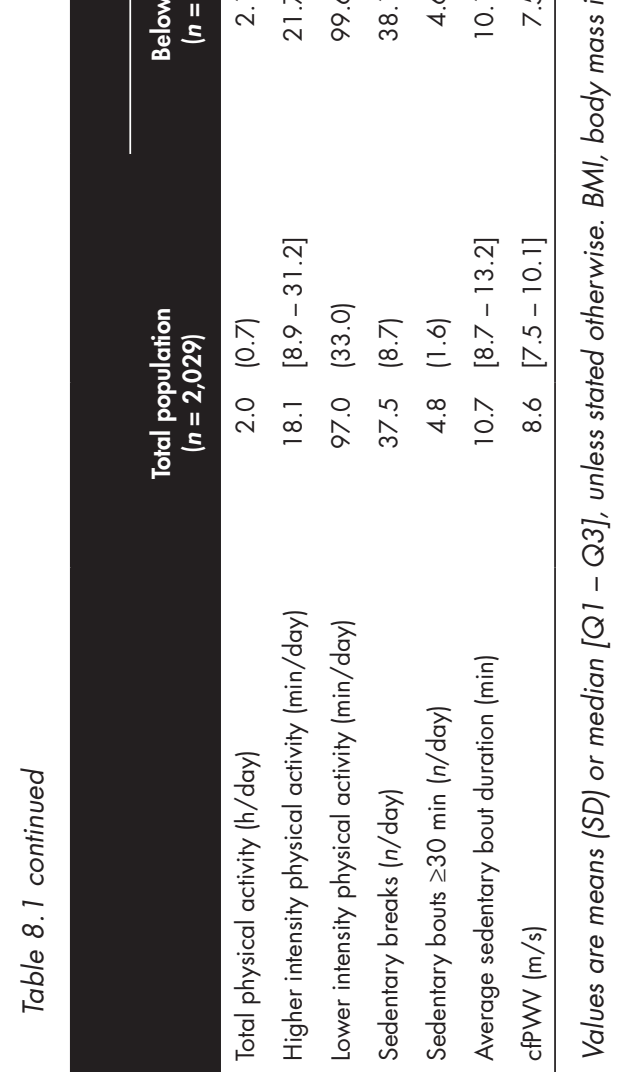

.

के

$\frac{\mathrm{m}}{\mathrm{D}}$

$\frac{\substack{0 \\ 0}}{0}$ 
Figure 8.1 shows the mean cfPWV according to the tertiles of total physical activity and sedentary behaviour, adjusted for sex, age and glucose metabolism status. CfPWV was $9.0 \mathrm{~m} / \mathrm{s}$ in participants with the smallest amount of total physical activity per day, while a cfPWV of $8.7 \mathrm{~m} / \mathrm{s}$ was observed in participants with the largest amount of total physical activity per day. In participants with the smallest amount of sedentary time per day cfPWV was $8.7 \mathrm{~m} / \mathrm{s}$. A higher cfPWV $(8.9 \mathrm{~m} / \mathrm{s})$ was observed in participants with the largest amount of sedentary time per day.

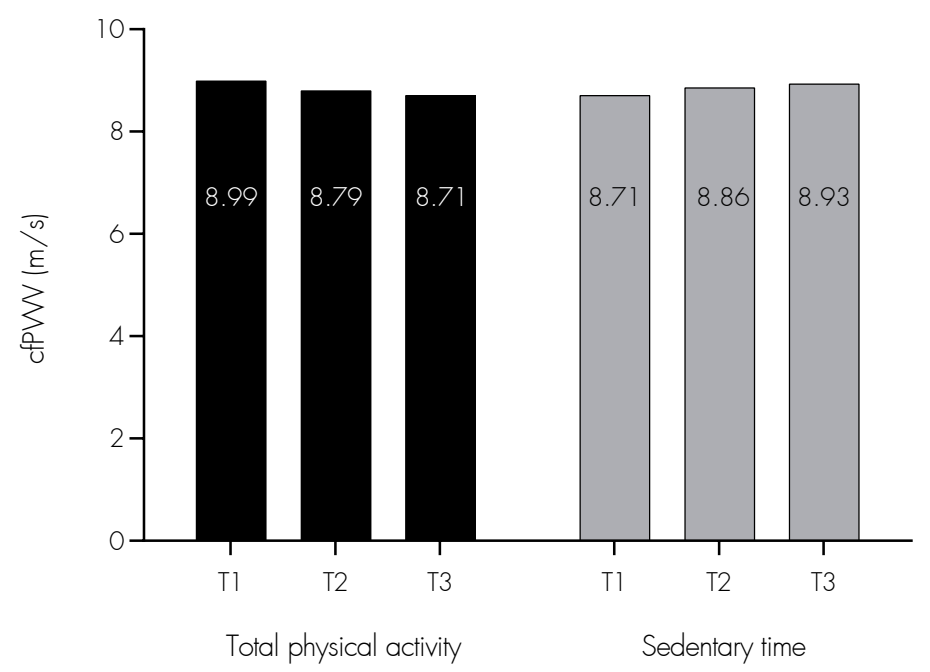

Figure 8.1: Carotid-to-femoral pulse wave velocity (cfPWV) according to the tertiles (T) of total physical activity and sedentary time. Data are mean values back transformed from natural log scale, adjusted for sex, age, waking time, and glucose metabolism status. Ranges of the tertiles were for total physical activity (h/day): 0.2-1.7, 1.7-2.3, 2.3-5.4; for sedentary time (h/day): 3. 1-8.7, 8.7-10.2, 10.2-15.9.

Table 8.2 shows the associations between the physical activity variables and cfPWV. Larger amounts of total physical activity were statistically significantly associated with a lower cfPWV after adjustment for potential confounders in model $2(B=0.986 ; 195 \% \mathrm{Cl}=$ $0.974 ; 0.998) 1$. Each extra 30 minutes of HPA per day was associated with a 0.98 times lower cfPWV, after adjustment for confounders and sedentary time (model 3; $B=0.982$; (0.969; 0.996)). In contrast, no significant association was observed between LPA and cfPWV. 
Table 8.2: Associations of physical activity variables with carotid to femoral pulse wave velocity

\begin{tabular}{|c|c|c|c|c|c|c|}
\hline & \multicolumn{2}{|r|}{ Model 1} & \multicolumn{2}{|r|}{ Model 2} & \multicolumn{2}{|r|}{ Model 3} \\
\hline & B & $(95 \% \mathrm{Cl})$ & B & $(95 \% \mathrm{Cl})$ & B & $(95 \% \mathrm{Cl})$ \\
\hline $\begin{array}{l}\text { Total physical activity } \\
\text { (h/day) }\end{array}$ & 0.979 & $(0.967 ; 0.991)$ & 0.986 & $(0.974 ; 0.998)$ & & - \\
\hline $\begin{array}{l}\text { Higher intensity physical } \\
\text { activity ( } 30 \mathrm{~min} / \text { day) }\end{array}$ & 0.970 & $(0.957 ; 0.984)$ & 0.980 & $(0.968 ; 0.993)$ & 0.982 & $(0.969 ; 0.966)$ \\
\hline $\begin{array}{l}\text { Lower intensity physical } \\
\text { activity ( } 30 \mathrm{~min} / \text { day) }\end{array}$ & 0.993 & $(0.985 ; 1.001)$ & 0.996 & $(0.989 ; 1.003)$ & & - \\
\hline
\end{tabular}

Regression results are presented as unstandardized coefficients (B), back-transformed from the natural log scale, with $95 \%$ confidence intervals (95\% CI). Boldface indicates statistical significance $(P<0.05)$. The associations in models 1 were adjusted for sex, age, waking time, and glucose metabolism status. The associations in models 2 were additionally adjusted for mean arterial pressure, heart rate, level of education, smoking status, alcohol consumption, body mass index, total-to-HDL cholesterol ratio, triglycerides, history of cardiovascular disease, use of anti-hypertensive medication, use of lipid-modifying medication, and mobility limitation. The association in model 3 was additionally adjusted for sedentary time.

Table 8.3 shows the associations between the sedentary behaviour variables and cfPWV. Larger amounts of sedentary time per day were statistically significantly associated with a higher cfPWV in model $1(B=1.007 ;(1.002 ; 1.013))$, but after adjustment for confounding factors in model 2, no significant associations were observed. In none of the models was the number of sedentary breaks per day significantly associated with CPPWV. The number of prolonged sedentary bouts per day was statistically significantly associated with a higher cfPWV in model $1(B=1.006 ;(1.000 ; 1.011))$, but after additional adjustment for relevant factors in model 2, no significant associations were observed. Having a longer average sedentary bout duration was also statistically significantly associated with a higher cfPWV $(B=1.003 ;(1.000 ; 1.005))$ in model 2 , but after additional adjustment for HPA and sedentary time in model 3, a significant association was no longer observed.

Additional analyses examining the number of sedentary breaks with a duration of at least $1 \mathrm{~min}$ or the number of sedentary break per sedentary hour resulted in similar findings (data not shown). Further, results were similar when the analyses were repeated using renin-angiotensin-aldosterone system inhibitors instead of antihypertensive medication or when we excluded participants with $<4$ valid days $(\geq 10 \mathrm{~h}$ of waking data). In addition, there was no interaction with glucose metabolism status for any of the analyses $(P>0.10)$. 
Table 8.3: Associations of sedentary behaviour variables with carotid to femoral pulse wave velocity

\begin{tabular}{|c|c|c|c|c|c|c|}
\hline & \multicolumn{2}{|c|}{ Model 1} & \multicolumn{2}{|r|}{ Model 2} & \multicolumn{2}{|r|}{ Model 3} \\
\hline & B & $(95 \% \mathrm{Cl})$ & B & $(95 \% \mathrm{Cl})$ & B & $(95 \% \mathrm{Cl})$ \\
\hline Sedentary time (h/day) & 1.007 & $(1.002 ; 1.013)$ & 1.005 & $(1.000 ; 1.010)$ & 1.003 & $(0.998 ; 1.009)$ \\
\hline Sedentary breaks (10/day) & 1.000 & $(0.990 ; 1.010)$ & 1.002 & $(0.993 ; 1.011)$ & 1.004 & $(0.995 ; 1.013)$ \\
\hline $\begin{array}{l}\text { Prolonged sedentary bout } \\
(\geq 30 \mathrm{~min})(\mathrm{n} / \text { day) }\end{array}$ & 1.006 & $(1.000 ; 1.011)$ & 1.004 & $(0.999 ; 1.010)$ & 1.001 & $(0.993 ; 1.009)$ \\
\hline $\begin{array}{l}\text { Average sedentary bout } \\
\text { duration (min) }\end{array}$ & 1.004 & $(1.001 ; 1.006)$ & 1.003 & $(1.000 ; 1.005)$ & 1.002 & $(0.999 ; 1.004)$ \\
\hline
\end{tabular}

Regression results are presented as unstandardized coefficients (B), back-transformed from the natural log scale, with $95 \%$ confidence intervals $(95 \% \mathrm{CI})$. Boldface indicates statistical significance $(P<0.05)$. The associations in models 1 were adjusted for sex, age, waking time, and glucose metabolism status. The associations in models 2 were additionally adjusted for mean arterial pressure, heart rate, level of education, smoking status, alcohol consumption, body mass index, total-to-HDL cholesterol ratio, triglycerides, history of cardiovascular disease, use of anti-hypertensive medication, use of lipid-modifying medication, and mobility limitation. The associations in models 3 were additionally adjusted for higher intensity physical activity. Sedentary breaks, prolonged sedentary bouts and average sedentary bout duration were also adjusted for sedentary time in models 3.

\section{DISCUSSION}

To our knowledge, this is the first study that has used posture-based accelerometry data to measure daily amounts and patterns of physical activity and sedentary behaviour in order to examine their associations with cfPWV. The results show that total physical activity and HPA, but not LPA, were associated with aortic stiffness after adjustment for potential confounders. Each extra hour of total physical activity was associated with 0.99 times lower cfPWV. Each extra 30 minutes of HPA was associated with a 0.98 times lower cfPWV, independent of sedentary time. Total physical activity as well as HPA may therefore be relevant targets for strategies aiming to prevent aortic stiffness and cardiovascular disease. The total amount of sedentary time, the number of long sedentary bouts and average sedentary bout duration were associated with a higher cfPWV, but these associations were no longer statistically significant after adjustment for potential confounders. The number of sedentary breaks was not associated with cfPWV.

To date only a few observational studies on objectively measured daily activity levels and aortic stiffness have been published. In line with our results, most studies reported positive associations between physical activity executed at higher intensity levels (total, 
moderate, vigorous) and arterial stiffness, while associations with lower intensity physical activity were not demonstrated.9-12,31 This was also seen in studies using self-reported physical activity levels ${ }^{32,33}$ and intervention studies. ${ }^{6}$ Mechanisms that could explain associations between HPA and PWV include direct effects via changes in vascular structure and function that affect blood pressure and heart rate, ${ }^{34-36}$ and indirect effects via processes such inflammation and insulin sensitivity. ${ }^{37}$ The intensity of physical activity seems to be important for these mechanisms, as associations with cfPWV were not observed for LPA.

Reported results on the amount of sedentary time and PWV have been less consistent. Some studies did show associations, ${ }^{10,12,13}$ while we and others did not.9,11 A possible explanation for these findings could lie in the different statistical approaches of these studies: our analyses were adjusted for a series of potential confounders, while other studies reported results of univariate analyses or the analyses were adjusted, but not for relevant factors such as accelerometer wear time. Further, because our study population was relatively homogeneous as the median cfPWV was $8.6 \mathrm{~m} / \mathrm{s}[\mathrm{Q}]-\mathrm{Q} 3=7.5-10.1$ $\mathrm{m} / \mathrm{s}$ ] and also the range of sedentary time was small [Q1-Q3 $=8.2-10.6 \mathrm{~h} /$ day], possible associations may not have been detected. Only one study has focused on sedentary behaviour patterns next to the amount of sedentary time. ${ }^{23}$ In line with our findings, associations of total amount of sedentary time and the number of sedentary bouts $\geq 10 \mathrm{~min}$ with parameters of arterial stiffness were not observed, although PWV was not included in this study. The study did report significant associations between the number of sedentary breaks per sedentary hour and some parameters of arterial stiffness, such as ambulatory arterial stiffness index and pulse pressure. However, sedentary breaks were determined with a waist-worn ActiGraph GT3X accelerometer (ActiGraph, Shalimar, FL, USA). It has been shown that this method overestimates the number of breaks due to misclassification of standing time as sedentary time because classification is solely based on acceleration data. $^{24,38}$ The favourable associations of sedentary breaks with parameters of arterial stiffness may therefore be due to more activity instead of the pattern in which sedentary time was accumulated. The activPAL accelerometer used in our study classified sedentary time based on both acceleration and posture and this method has shown to be highly accurate for estimating sedentary time and breaks. ${ }^{24,25,38}$ Taken together, the inconsistency between the studies could have been caused by the different adjustment strategies, which varied from univariate analyses to adjustment for a series of potential confounders. Furthermore, the use of several different methods to estimate sedentary time, which varied 
from 'time being inactive including sleep' to '<100 counts/min' or ' $<100$ counts/30 sec' and posture-based classification, may also have contributed to the divergent results. In order to increase comparability between studies, future studies should use similar measures for both physical activity and sedentary behaviour constructs, and ideally adjust for similar confounding factors.

A major strength of our study was the use of posture-based accelerometry data to measure habitual physical activity and sedentary behaviour levels and patterns. This method has shown to be a highly accurate method to measure sedentary behaviour, ${ }^{24,25}$ thus our estimates of the amount of sedentary time and the determination of sedentary breaks are more accurate than those based solely on acceleration. Further the waterproof attachment of the accelerometer enabled us to collect $24 \mathrm{~h}$ accelerometry data which resulted in complete data assessments and which could have resulted in improved wear time compliance. ${ }^{39}$ In addition, we assessed aortic stiffness by cfPWV, which is considered as the gold standard measurement. ${ }^{5}$ Another strength was the adjustment for a series of potential confounders, including HPA and sedentary time. However, after adjustment for potential confounders in models 2 the associations of sedentary time and prolonged sedentary bouts with cfPWV were nonsignificant. The differences between models 1 and 2 were primarily caused by BMI, heart rate, and blood pressure. Since some of the potential confounders, such as blood pressure, may also be part of the pathway between sedentary behaviour and cfPWV, the analyses could have been subject to overadjustment. ${ }^{40} \mathrm{Also}$, the analyses with the physical activity variables, sedentary breaks, and sedentary bout duration may have been subject to overadjustment for the same reason.

Our study has also limitations which should be considered. The most important limitation is the cross-sectional nature of our analyses; causal relationships could therefore not be determined. Nevertheless, several randomized controlled trials have demonstrated positive relations between physical exercise and $P W V,{ }^{6}$ which suggest that the direction of the association is from physical activity to aortic stiffness. In addition, due to missing data, we had to exclude 1,400 participants. However, the excluded participants did not differ from our study sample with regard to demographic factors, accelerometry variables and cfPWV (data not shown). Further, the accelerometer was worn during one week only, which may not truly reflect habitual behaviour. In addition, our accelerometry data was posture-based, so the determination of higher and lower intensity physical activity was based on step frequency. This method is less precise than using acceleration to determine intensity levels, although it has been demonstrated that a step frequency of $>\sim 100$ steps/ 
min equals a MET score of $\geq 3.0^{29}$ and we used a cut-off point of $>110$ steps $/ \mathrm{min}$ for higher intensity physical activity. Finally, as our study population consisted of a relatively healthy population with well-controlled participants with T2DM (median HbAlc $(\%)=6.7$ $[Q 1-Q 3=6.3-7.2])$, the results might not representative for the general population of adults aged $40-75$ years.

To conclude, this large study that has used posture-based accelerometry data to assess amounts and patterns of all daily activity showed that larger amounts of total physical activity and HPA were associated with lower cFPWV. The amount and pattern of sedentary behaviour were in our population not associated with aortic stiffness. In order to confirm our results and compare results of studies using accelerometry data, future (longitudinal) studies are encouraged to use similar definitions of accelerometry constructs and adjust for similar relevant factors. Together with our study, these studies will contribute to a better understanding of the associations between physical activity and sedentary behaviour with aortic stiffness, which is of interest from both an aetiological and public health point of view. 


\section{REFERENCES}

1. Ben-Shlomo $Y$, Spears M, Boustred C, May M, Anderson SG, Benjamin EJ, et al. Aortic pulse wave velocity improves cardiovascular event prediction. J Am Coll Cardiol. 2014;63(7):636-46.

2. Vlachopoulos C, Aznaouridis K, Stefanadis C. Prediction of cardiovascular events and all-cause mortality with arterial stiffness. J Am Coll Cardiol. 2010;55(13):1318-27.

3. Benetos A, Waeber B, Izzo J. Influence of age, risk factors, and cardiovascular and renal disease on arterial stiffness: Clinical applications. Am J Hypertens. 2002;15(12):1101-8.

4. Zieman SJ. Mechanisms, pathophysiology, and therapy of arterial Stiffness. Arterioscler Thromb Vasc Biol. 2005;25(5):932-43.

5. Laurent S, Cockcroft J, Van Bortel L, Boutouyrie P, Giannattasio C, Hayoz D, et al. Expert consensus document on arterial stiffness: Methodological issues and clinical applications. Eur Heart J. 2006;27(21):2588-605.

6. Ashor AW, Lara J, Siervo M, Celis-Morales C, Mathers JC. Effects of exercise modalities on arterial stiffness and wave reflection: A systematic review and meta-analysis of randomized controlled trials. PLoS One. 2014;9(10):e1 10034.

7. Ainsworth BE, Haskell WL, Whitt MC, Irwin ML, Swartz ANNM, Strath SJ, et al. Compendium of physical activities: An update of activity codes and MET intensities. Med Sci Sports Exerc. 2000;32(Suppl 9):S498-504.

8. Troiano RP, Berrigan D, Dodd KW, Mâsse LC, Tilert T, McDowell M. Physical activity in the United States measured by accelerometer. Med Sci Sports Exerc. 2008;40(1):181-8.

9. Andersson C, Lyass A, Larson MG, Spartano NL, Vita JA, Benjamin EJ, et al. Physical activity measured by accelerometry and its associations with cardiac structure and vascular function in young and middle-aged adults. J Am Heart Assoc. 2015;4(3):e001528-e001528.

10. Gando Y, Yamamoto K, Murakami H, Ohmori $Y$, Kawakami R, Sanada K, et al. Longer time spent in light physical activity is associated with reduced arterial stiffness in older adults. Hypertension. 2010;56(3):540-6.
11. Gomez-Marcos MA, Recio-Rodríguez JI, PatinoAlonso MC, Agudo-Conde C, Lasaosa-Medina $L$, Rodriguez-Sanchez E, et al. Relationship between objectively measured physical activity and vascular structure and function in adults. Atherosclerosis. 2014;234(2):366-72.

12. Horta BL, Schaan BD, Bielemann RM, Vianna CÁ, Gigante DP, Barros FC, et al. Objectively measured physical activity and sedentarytime are associated with arterial stiffness in Brazilian young adults. Atherosclerosis. $2015 ; 243(1): 148-54$.

13. Mac Ananey $O$, Mcloughlin B, Leonard A, Maher L, Gaffney P, Boran G, et al. Inverse relationship between physical activity, adiposity and arterial stiffness in healthy middle-aged subjects. J Phys Act Health. 2015; 12(12):1576-81.

14. Matthews CE, Chen KY, Freedson PS, Buchowski MS, Beech M, Pate RR, et al. Amount of time spent in sedentary behaviors in the United States, 2003-2004. Am J Epidemiol. 2008; 167(7):875-81.

15. Sedentary Behaviour Research Network. Standardized use of the terms "sedentary" and "sedentary behaviours." Appl Physiol Nutr Metab. 2012;37:540-2.

16. Brocklebank LA, Falconer CL, Page AS, Perry R, Cooper AR. Accelerometer-measured sedentary time and cardiometabolic biomarkers: A systematic review. Prev Med. 2015;76:92-102.

17. Healy GN, Matthews CE, Dunstan DW, Winkler EAH, Owen N. Sedentary time and cardiometabolic biomarkers in US adults: NHANES 2003-06. Eur Heart J. 2011 1;32(5):590-7.

18. Atkin A, Gorely T, Clemes SA, Yates T, Edwardson C, Brage S, et al. Methods of measurement in epidemiology: Sedentary behaviour. Int J Epidemiol. 2012;41(5):1460-71.

19. Healy G, Dunstan D, Salmon J, Cerin E, Shaw J, Zimmet $P$, et al. Breaks in sedentary time. Diabetes Care. 2008;31 (4):661-3.

20. Benatti F, Ried-Larsen M. The effects of breaking up prolonged sitting time. Med Sci Sport Exerc. 2015;47(10):2053-61

21. Cooper AR, Sebire S, Montgomery AA, Peters TJ, Sharp DJ, Jackson N, et al. Sedentary time, breaks in sedentary time and metabolic variables in people with newly diagnosed type 2 diabetes. Diabetologia. 2012;55(3):589-99. 
22. Gennuso KP, Gangnon RE, Thraen-Borowski KM, Colbert LH. Dose-response relationships between sedentary behaviour and the metabolic syndrome and its components. Diabetologia. 2014;58:485-92.

23. García-Hermoso A, Notario-Pacheco B, RecioRodríguez Jl, Martínez-Vizcaíno V, Rodrigo de Pablo E, Magdalena Belio JF, et al. Sedentary behaviour patterns and arterial stiffness in a Spanish adult population - The EVIDENT trial. Atherosclerosis. 2015;243(2):516-22.

24. Kozey-Keadle S, Libertine A, Lyden K, Staudenmayer J, Freedson PS. Validation of wearable monitors for assessing sedentary behavior. Med Sci Sport Exerc. $2011 ; 43(8): 1561-7$.

25. Godfrey A, Culhane KM, Lyons GM. Comparison of the performance of the activPAL ${ }^{\mathrm{TM}}$ Professional physical activity logger to a discrete accelerometer-based activity monitor. Med Eng Phys. 2007;29(8):930-4.

26. Schram MT, Sep SJS, van der Kallen CJ, Dagnelie PC, Koster A, Schaper N, et al. The Maastricht Study: An extensive phenotyping study on determinants of type 2 diabetes, its complications and its comorbidities. Eur J Epidemiol. 2014;29(6):439-51.

27. Van Bortel LM, Laurent $S$, Boutouyrie $P$, Chowienczyk P, Cruickshank JK, De Backer $\mathrm{T}$, et al. Expert consensus document on the measurement of aortic stiffness in daily practice using carotid-femoral pulse wave velocity. J Hypertens. 2012;30(3):445-8.

28. Van der Berg J, Willems P, Van der Velde J, Savelberg H, Schaper N, Schram M, et al. Identifying waking time in 24-h accelerometry data in adults using an automated algorithm. J Sports Sci. 2016;2:1-7.

29. Tudor-Locke C, Craig CL, Brown WJ, Clemes SA, De Cocker K, Giles-Corti B, et al. How many steps/day are enough? For adults. Int J Behav Nutr Phys Act. $2011 ; 8(1): 79$.

30. Tudor-Locke C, Rowe DA. Using cadence to study free-living ambulatory behaviour. Sports Med. 2012;42(5):381-98.

31. García-Ortiz L, Recio-Rodríguez JI, SchmidtTrucksäss A, Puigdomenech-Puig E, MartínezVizcaíno V, Fernández-Alonso $C$, et al. Relationship between objectively measured physical activity and cardiovascular aging in the general population - The EVIDENT trial. Atherosclerosis. 2014;233(2):434-40.

32. Van de Laar RJ, Ferreira I, van Mechelen W, Prins MH, Twisk JW, Stehouwer CD. Lifetime vigorous but not light-to-moderate habitual physical activity impacts favorably on carotid stiffness in young adults: The Amsterdam Growth and Health Longitudinal Study. Hypertension. 2010;55(1):33-9

33. Boreham CA, Ferreira I, Twisk JW, Gallagher AM, Savage M, Murray L. Cardiorespiratory fitness, physical activity, and arterial stiffness: The Northern Ireland young hearts project. Hypertension. 2004;44:721-6.

34. Gielen S, Schuler G, Adams V. Cardiovascular effects of exercise training: Molecular mechanisms. Circulation. 2010;122(12):122138 .

35. Green DJ. Exercise training as vascular medicine: Direct impacts on the vasculature in humans. Exerc Sport Sci Rev. 2009;37(10):196-202.

36. Thijssen DHJ, Maiorana AN, O'Driscoll G, Cable NT, Hopman MTE, Green DJ. Impact of inactivity and exercise on the vasculature in humans. Eur J Appl Physiol. 2010;108(5):845-75.

37. Teixeira-Lemos E, Nunes S, Teixeira F, Reis F. Regular physical exercise training assists in preventing type 2 diabetes development: Focus on its antioxidant and anti-inflammatory properties. Cardiovasc Diabetol. 2011;10(1):12

38. Lyden K, Kozey Keadle SL, Staudenmayer JW, Freedson PS. Validity of two wearable monitors to estimate breaks from sedentary time. Med Sci Sports Exerc. 2012;44(1 1):2243-52.

39. Tudor-Locke C, Barreira TV, Schuna JM, Mire $E F$, Chaput J-P, Fogelholm $M$, et al. Improving wear time compliance with a 24-hour waistworn accelerometer protocol in the International Study of Childhood Obesity, Lifestyle and the Environment (ISCOLE). Int J Behav Nutr Phys Act. 2015;12(11):11.

40. Schisterman EF, Cole SR, Platt RW

Overadjustment bias and unnecessary adjustment in epidemiologic studies. Epidemiology. 2009;20(4):488-95. 

Chapt

照 

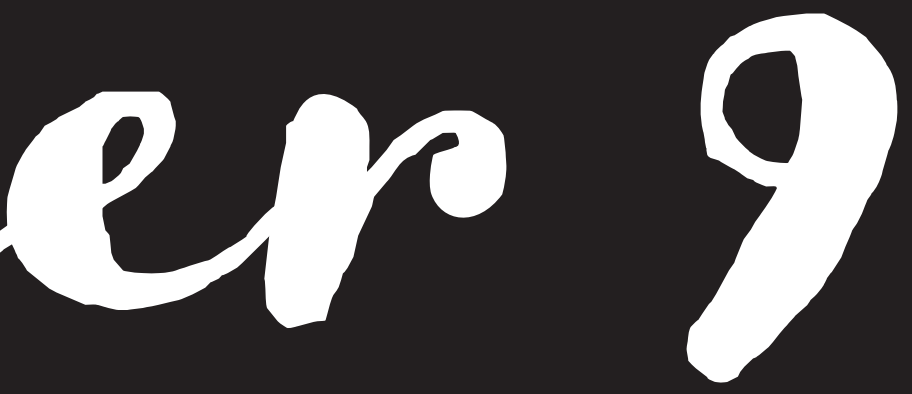

Associations of total amount and patterns of physical activity and sedentary behaviour with estimated glomerular filtration rate and albuminuria: The Maastricht Study

REMY J.H. MARTENS* | JULIANNE D. VAN DER BERG* | COEN D.A. STEHOUWER | RONALD M.A. HENRY | HANS BOSMA | PIETER C. DAGNELIE | MARTIEN C.J.M. VAN DONGEN | SIMONE J.P.M. EUSSEN | MIRANDA T. SCHRAM | SIMONE J.S. SEP | CARLA J.H. VAN DER KALLEN | NICOLAAS C. SCHAPER | HANS H.C.M. SAVELBERG | FRANK M. VAN DER SANDE | ABRAHAM A. KROON | JEROEN P. KOOMAN ${ }^{\dagger}$ | ANNEMARIE KOSTER ${ }^{\dagger}$ 


\section{AB STR ACT}

Background: Reduced estimated glomerular filtration rate (eGFR) and albuminuria have become highly prevalent and both have been associated with renal and cardiovascular disease risk. Thus, it is important to identify modifiable risk factors for reduced eGFR and albuminuria. Therefore, we examined associations of total amount and patterns of physical activity and sedentary behaviour with eGFR and albuminuria.

Methods: We included 2,258 participants (51\% men, age (mean \pm SD): $60.1 \pm 8.1$ years) of The Maastricht Study, who were asked to wear an accelerometer $24 \mathrm{~h} /$ day on 8 consecutive days. We calculated daily amounts of total physical activity (TPA), higher intensity physical activity (HPA), lower intensity physical, activity (LPA), and sedentary time; daily numbers of sedentary breaks and prolonged sedentary bouts ( $\geq 30 \mathrm{~min}$ ); and average sedentary bout duration. Multivariable linear regression analyses were used to examine associations between accelerometry variables and eGFR crys $_{\text {i }}$ multinomial logistic regression analyses were used to examine the associations with categories of urinary albumin excretion (UAE; $<15,<30, \geq 30 \mathrm{mg} / 24 \mathrm{~h}$ ).

Results: After adjustment for potential confounders, each extra hour of TPA was associated with a higher eGFR $(B=2.30(95 \% \mathrm{Cl}=1.46$; 3.14)), whereas each extra hour of sedentary time was associated with a lower eGFR ( $B=-0.71(-1.08 ;-0.35))$. Having more prolonged sedentary bouts and having a longer average sedentary bout duration were associated with a lower eGFR. Further, an extra hour of TPA was associated with a lower odds for albuminuria $\left(O R_{<30 \mathrm{mg} / 24 \mathrm{~h}}=0.63(0.41\right.$, $0.96), \mathrm{OR}_{\geq 30 \mathrm{mg} / 24 \mathrm{~h}}=0.84(0.53,1.35)$ (highest vs lowest quartile of TPA)). An extra hour of sedentary time was associated with a higher odds for albuminuria $\left(O R_{<30 \mathrm{mg} / 24 \mathrm{~h}}=1.11(1.01,1.22)_{,} O R_{\geq 30 \mathrm{mg} / 24 \mathrm{~h}}=1.10\right.$ $(0.99,1.22))$. No associations were observed between patterns of sedentary behaviour and albuminuria.

Discussion: Both TPA and sedentary behaviour were associated with eGFR $_{\text {crys }}$ and albuminuria, and also the sedentary behaviour pattern

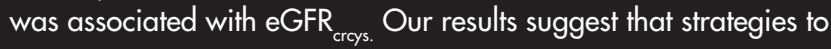
prevent reduced eGFR and albuminuria should focus not only on increasing physical activity levels, but on reducing sedentary time as well. 


\section{INTRODUCTION}

Reduced estimated glomerular filtration rate (eGFR) and albuminuria, which together define chronic kidney disease (CKD), ${ }^{1}$ have become highly prevalent in modern Western society. ${ }^{2}$ Both eGFR and albuminuria have been associated with end-stage renal disease and cardiovascular disease (CVD) risk. ${ }^{3}$ Therefore, it is important to identify factors that contribute to the development of reduced eGFR and albuminuria.

A possibly important modifiable factor is the level of physical activity, which includes not only moderate to vigorous physical activity (MVPA) like brisk walking or running, but also light intensity physical activity such as casual walking and household work. ${ }^{4}$ Further, sedentary behaviour, such as watching TV, using the computer or driving, could be an important factor that should be examined next to physical activity, as adults spend most time of the day being sedentary. ${ }^{5.7}$ Several studies have reported on associations of physical activity ${ }^{8-15}$ and sedentary behaviour ${ }^{11,16-19}$ with eGFR, 8, 11,14-16, 18,19 albuminuria, ${ }^{9-13,16,19}$ or both combined as CKD. ${ }^{11,17}$ However, these studies have used selfreporting measures, which may be biased and the reported results have been inconsistent.

Since several years the complete daily activity spectrum, from sedentary behaviour to high intensity physical activity, can be objectively measured with an accelerometer, but to date only a few studies have described associations between accelerometry data and kidney function. ${ }^{20,21}$ Additionally, these studies did not report on the pattern of sedentary time, i.e. the manner in which sedentary time was accumulated. This can be expressed by sedentary breaks, which refers to the frequency with which sedentary time was interrupted (e.g. transitions from sitting to standing) or sedentary bouts, which refers to uninterrupted periods of sedentary time of a certain duration (e.g. $30 \mathrm{~min}$ ). Previous studies have demonstrated that the pattern of sedentary time was associated with detrimental health outcomes, including an increased waist circumference and BMI, higher levels of triglycerides and glucose, and the metabolic syndrome. ${ }^{22-26}$

In view of the above, we measured both total amount and patterns of physical activity and sedentary behaviour in a large sample of adults aged 40-75 years to examine their associations with eGFR and albuminuria. 


\section{METHODS}

\section{Study population}

We used data of The Maastricht Study, an observational prospective population-based cohort study. The rationale and methodology have been described previously. ${ }^{27}$ In brief, the study focuses on the aetiology, pathophysiology, complications and comorbidities of T2DM and is characterized by an extensive phenotyping approach. Eligible participants were individuals aged between 40 and 75 years and living in the southern part of the Netherlands. Participants were recruited through mass media campaigns and from the municipal registries and the regional Diabetes Patient Registry via mailings. Recruitment was stratified according to known T2DM status, with an oversampling of individuals with T2DM, for reasons of efficiency. The present report includes cross-sectional data from the first 3,451 participants, who completed the baseline survey between November 2010 and September 2013. Participants with type 1 diabetes, latent auto-immune diabetes in adults (LADA), steroid-induced diabetes or diabetes after pancreatectomy $(n=41)$ were excluded. After further successively excluding participants who did not receive an accelerometer due to logistics ( $n=668$ ) or whose accelerometer measurement failed ( $n=135)$, whose eGFR was missing $(n=20)$, whose $24 \mathrm{~h}$ urine collections were either collected erroneously $(<20$ $\mathrm{h}$ or $>28 \mathrm{~h})$ or were not handed in at all $(n=23)$ or who had other missing data $(n=306)$, a total of 2,258 participants were included in the present study.

The study has been approved by the institutional medical ethical committee (NL3 1329.068.10) and the Minister of Health, Welfare and Sports of the Netherlands (Permit 131088-105234-PG), and was conducted in accordance with the Declaration of Helsinki. All participants gave written informed consent.

\section{Measurements}

Kidney function

GFR was estimated with the CKD-EPI equation based on the combination of serum creatinine and serum cystatin $C\left(\right.$ GGFR $\left._{\text {crys }}\right){ }^{28}$ Serum creatinine was measured with a Jaffé method traceable to isotope dilution mass spectrometry (due to a change of supplier by the Beckman Synchron LX20, Beckman Coulter, Brea, USA and the Roche Cobas 6000, F. Hoffmann-La Roche, Basel, Switzerland). Serum cystatin C was measured with a particle enhanced immunoturbidimetric assay standardized against ERM-DA471/IFCC reference material (Roche Cobas 8000, F. Hoffman-La Roche, Basel, Switzerland). 
To assess urinary albumin excretion (UAE), participants were requested to collect two $24 \mathrm{~h}$ urine collections. Urinary albumin concentration was measured with a standard immunoturbidimetric assay by an automatic analyser (due to a change of supplier by the Beckman Synchron LX20, Beckman Coulter, Brea, USA and the Roche Cobas 6000, F. Hoffmann-La Roche, Basel, Switzerland) and multiplied by collection volume to obtain the $24 \mathrm{~h}$ UAE. A urinary albumin concentration below the detection limit of the assay (2 $\mathrm{mg} / \mathrm{L}$ for the Beckman Synchron LX20 and $3 \mathrm{mg} / \mathrm{L}$ for the Roche Cobas 6000) was set at $1.5 \mathrm{mg} / \mathrm{L}$ before multiplying by collection volume. Only urine collections with a collection time between $20 \mathrm{~h}$ and $28 \mathrm{~h}$ were considered valid. If needed, UAE was extrapolated to a $24 \mathrm{~h}$ excretion. UAE was expressed as categorical variable $/<15 \mathrm{mg} / 24 \mathrm{~h},<30 \mathrm{mg} / 24$ $\mathrm{h}$ and $\geq 30 \mathrm{mg} / 24 \mathrm{~h},{ }^{29}$ in agreement with the fact that an association with (cardiovascular) disease already exists below the clinical cut-off value of $\left.30 \mathrm{mg} / 24 \mathrm{~h}^{3}\right)$. These analyses were preferably based on the average of two (available in $92.6 \%$ of the participants) $24 \mathrm{~h}$ urine collections.

\section{Accelerometry variables}

Daily activity levels and patterns were measured using the activPAL3 ${ }^{\text {TM }}$ physical activity monitor (PAL Technologies, Glasgow, UK). The activPAL3 is a small (53 $\times 35 \times 7 \mathrm{~mm})$, lightweight $(15 \mathrm{~g})$ triaxial accelerometer that records movement in the vertical, anteroposterior and mediolateral axes, and also determines posture (sitting or lying, standing and stepping) based on acceleration information. The device was attached directly to the skin on the front of the right thigh with transparent $3 M$ Tegaderm $^{\mathrm{TM}}$ tape, after the device had been waterproofed using a nitrile sleeve. Participants were asked to wear the accelerometer for 8 consecutive days, without removing it at any time. To avoid inaccurately identifying non-wear time, participants were asked not to replace the device once removed. Data were uploaded using the activPAL software and processed using customizes software written in MATLAB R2013b (MathWorks, Natick, MA, USA). Data from the first day were excluded from the analysis because participants performed physical function tests at the research centre after the device was attached. In addition, data from the final wear day providing $\leq 14$ waking hours of data were excluded from the analysis. Participants were included if they provided at least 1 valid day ( $\geq 10 \mathrm{~h}$ of waking data).

The total amount of physical activity was based on the stepping posture and calculated as the mean time spent in stepping during waking time per day. The method used to determine waking time has been described elsewhere. ${ }^{30}$ Physical activity (stepping 
time) was further classified into higher intensity physical activity (HPA; minutes with a step frequency $>110$ steps/min during waking time $)^{31,32}$ and lower intensity physical activity (LPA; minutes with a step frequency $\leq 110$ steps/min during waking time. Standing time was not included.). The total amount of sedentary time was based on the sedentary posture (sitting or lying), and calculated as the mean time spent in a sedentary position during waking time per day. The number of sedentary breaks during waking time was determined as each transition from a sitting or lying position to standing or stepping with a duration of at least $1 \mathrm{~min}$, and the mean number of breaks per day was calculated. Sedentary time accumulated in a consecutive period $\geq 30 \mathrm{~min}$ was defined as a prolonged sedentary bout, and the mean number of prolonged sedentary bouts during waking time per day was calculated. Average bout duration was calculated by dividing total sedentary time by total number of sedentary bouts of any duration.

\section{Potential confounders or mediators}

Potential confounders or mediators which were extracted from questionnaires included sex, age, smoking status, alcohol consumption, daily energy intake, mobility limitation, comorbidity, and history of cardiovascular disease, whereas level of education and use of antihypertensive and lipid-modifying medication were assessed by interview. Smoking status was categorized into never, former and current smoker, and alcohol consumption into no consumption, low consumption $1 \leq 7$ glasses per week for women and $\leq 14$ glasses per week for men) and high consumption (>7 glasses per week for women and > 14 glasses per week for men). Energy intake was obtained from a food frequency questionnaire and calculated as the mean energy intake (kcal) per day. Mobility limitation was obtained from the EuroQol-5D questionnaire and was defined as having any difficulties with walking in the previous week. Comorbidity was determined by the presence of (a history of) one or more of the following conditions: non-skin cancer, inflammatory respiratory disease or Parkinson's disease. Participants without data on these conditions ( $n=12$ ) were classified as having no comorbid disease. Prevalent CVD was defined as a self-reported history of myocardial infarction, cerebrovascular infarction or haemorrhage, or percutaneous artery angioplasty of, or vascular surgery on, the coronary, abdominal, peripheral or carotid arteries. Level of education was categorized into low (none, primary, or lower vocational education only), medium (intermediate general secondary, intermediate vocational or higher general secondary education) and high (higher vocational education or university level of education). 
Other potential confounders or mediators were obtained from physical examination and laboratory assessment as described elsewhere, ${ }^{27}$ and included waist circumference, total cholesterol, HDL cholesterol, triglycerides, office blood pressure, and glucose metabolism status. Total-to-HDL cholesterol ratio was calculated by dividing total cholesterol by HDL cholesterol. Hypertension was defined as an office systolic blood pressure $\geq 140$ $\mathrm{mmHg}$, an office diastolic blood pressure $\geq 90 \mathrm{mmHg}$ and/or the use of antihypertensive medication. Glucose metabolism was classified according to the World Health Organization 2006 criteria $^{33}$ into normal glucose metabolism, impaired fasting glucose, impaired glucose tolerance, and diabetes mellitus. Participants with diabetes mellitus and participants using glucose-lowering medication were considered as having T2DM if they had no (self-reported) type 1 or other specific type of diabetes mellitus. For the present study, impaired fasting glucose and impaired glucose tolerance were combined into impaired glucose metabolism.

\section{Statistical analysis}

Characteristics of the total study population and according to sex-specific quartiles of sedentary time (to obtain equal distributions of men and women) were summarized as mean with standard deviations (SD) or as numbers and percentages. Non-normally distributed variables were described using the median and the interquartile range. General linear models were used to obtain adjusted means of physical activity and sedentary behaviour variables according to categories of eGFR crcys $_{\text {and }}$ albuminuria.

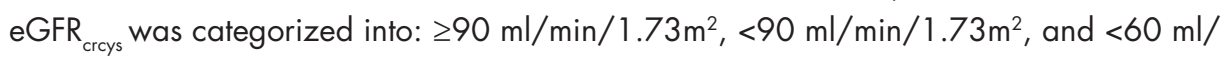
$\mathrm{min} / 1.73 \mathrm{~m}^{2}{ }^{1}$ Albuminuria was categorized into: $<15 \mathrm{mg} / 24 \mathrm{~h},<30 \mathrm{mg} / 24 \mathrm{~h}$, and $\geq 30$ $\mathrm{mg} / 24$ h. ${ }^{29}$

Associations of total physical activity (h/day), HPA (10 min/day), LPA (h/day), sedentary time (h/day), sedentary breaks (10/day), prolonged sedentary bouts ( $n /$ day), and average sedentary bout duration ( $\mathrm{min}$ ) with eGFR $_{\text {crys }}$ as dependent variable were evaluated with multivariable linear regression analyses. The regression coefficients (B) represent the difference in eGFR $_{\text {crys }}$ per one unit increase in the independent variable. The associations of the physical activity and sedentary behaviour variables with albuminuria were evaluated with categories of albuminuria using multinomial logistic regression analyses, because UAE was highly positively skewed and could not be transformed adequately using common ${ }^{34}$ transformations. Total physical activity and LPA were categorized into quartiles as the association with categorical albuminuria was 
non-linear. The odds ratios (OR) represent the odds of having a UAE of $<30 \mathrm{mg} / 24 \mathrm{~h}$ or a UAE of $\geq 30 \mathrm{mg} / 24 \mathrm{~h}$, respectively, as compared with the first quartile for total physical activity and LPA, or per one unit increase in HPA or the sedentary behaviour variables.

The associations in models 1 were adjusted for age, sex, glucose metabolism status, educational level, smoking status, alcohol consumption, energy intake, comorbid disease, mobility limitation, and waking time (to exclude the possibility that estimated effects were biased due to differences in waking hours). In models 2 the associations with the sedentary behaviour variables were additionally adjusted for HPA, to examine whether the effects of sedentary behaviour were independent of the amount of HPA. In models 3 the associations with HPA and the sedentary behaviour pattern variables were additionally adjusted for sedentary time, to examine whether the effects were independent of the amount of sedentary time. In models 4 all associations were additionally adjusted for potential mediators (or potential confounders) which included office systolic blood pressure, use of antihypertensive medication, waist circumference, total-to-HDL cholesterol ratio, triglycerides, use of lipid-modifying medication, and prevalent CVD. All analyses were performed with IBM SPSS Statistics Version 22.0 (IBM Corp., Armonk, NY, USA).

\section{RESULTS}

Table 9.1 presents descriptive characteristics of the total study population and according to sex-specific quartiles of sedentary hours per day. Of the total population, $51.3 \%$ was male and the average age was $60.1 \pm 8.1$ years. eGFR ${ }_{\text {crys }}$ was on average $87.9 \pm 14.6 \mathrm{ml} /$ $\mathrm{min} / 1.73 \mathrm{~m}^{2}$ in the total population, and $4.3 \%$ had an eGFR crcys $_{\text {of }}<60 \mathrm{ml} / \mathrm{min} / 1.73 \mathrm{~m}^{2}$. The median UAE was $6.6 \mathrm{mg} / 24 \mathrm{~h}$, and $8.8 \%$ of the total study population had a UAE of $\geq 30 \mathrm{mg} / 24 \mathrm{~h}$. The accelerometer was worn on average $6.3 \pm 1.2$ days. Average waking time was $15.7 \pm 0.9 \mathrm{~h} /$ day, of which most time was spent sedentary $(9.4 \pm 1.7 \mathrm{~h})$, and only $4.3 \pm 1.3 \mathrm{~h}$ and $2.0 \pm 0.7 \mathrm{~h}$ were spent standing and being physically active (stepping), respectively. The amount of LPA was on average $1.6 \pm 0.6 \mathrm{~h} /$ day, and the median HPA was $18.4 \mathrm{~min} /$ day [Q1-Q3 = $9.0-31.4]$. 


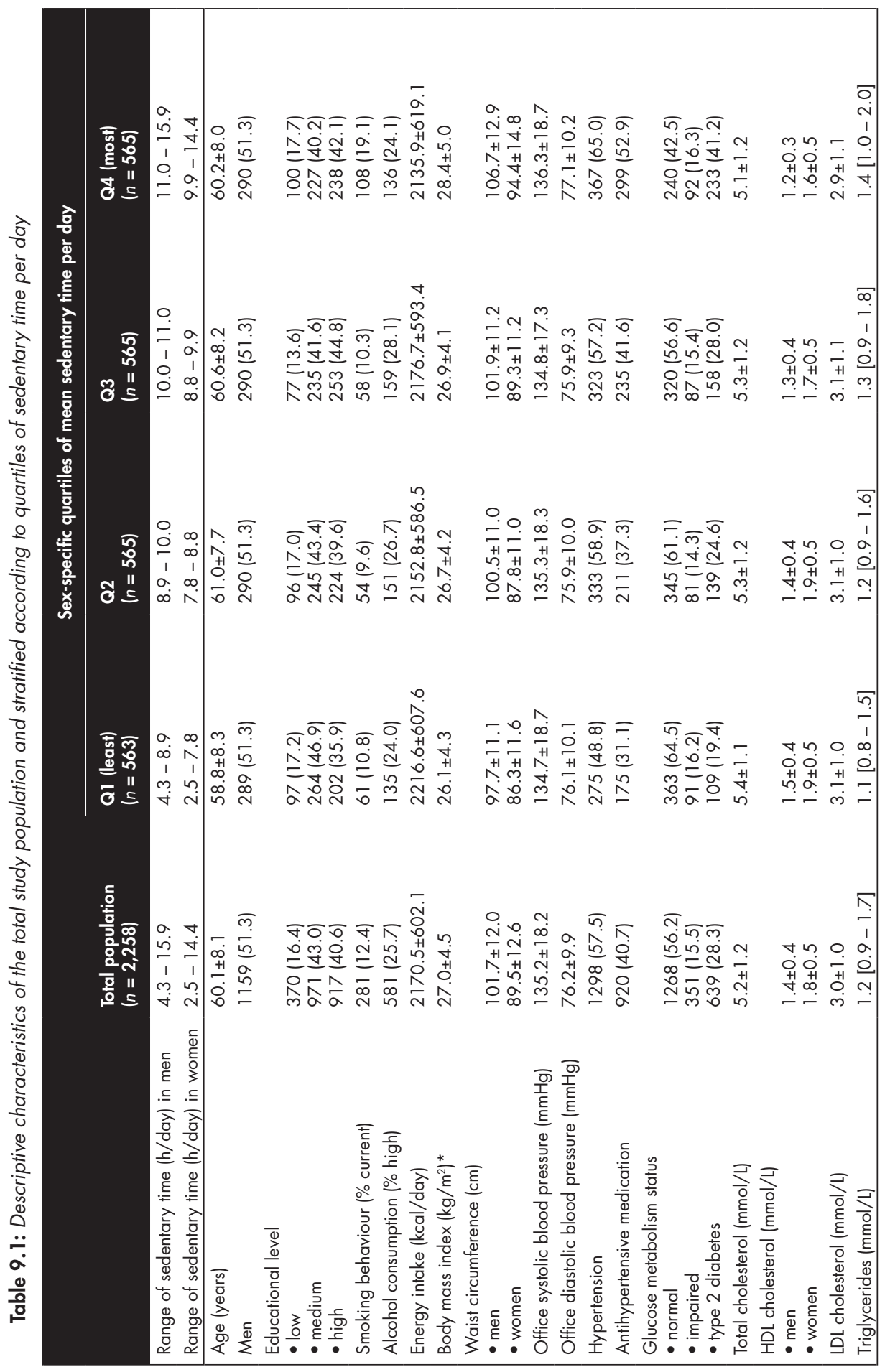


Figure 9.1 shows the daily percentages of sedentary time, standing time and time spent physically active (stepping) according to eGFR crcys $_{\text {and }}$ and albuminuria categories.

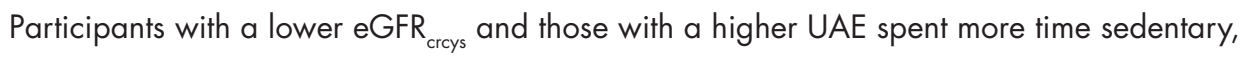
so consequently less time standing and physically active in comparison with those with a

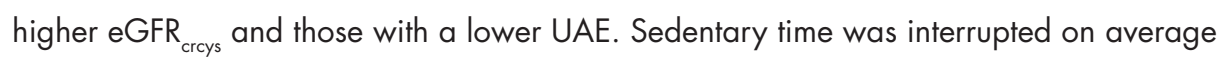
$37.4 \pm 8.5$ times per day (sedentary breaks). The daily number of prolonged sedentary bouts (accumulated in consecutive period $\geq 30 \mathrm{~min}$ ) was on average $4.9 \pm 1.6$, and the median average sedentary bout duration was $10.7 \mathrm{~min}$ [8.7 - 13.2] (Table 9.1).

A

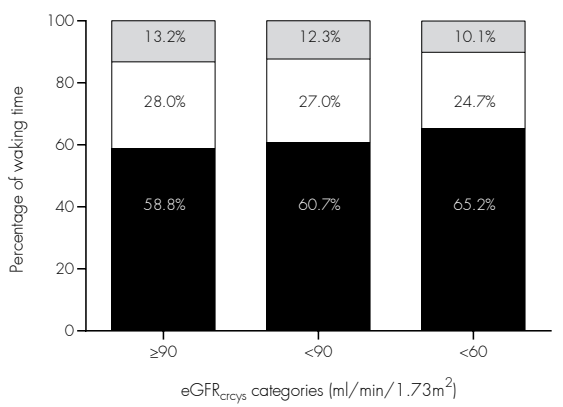

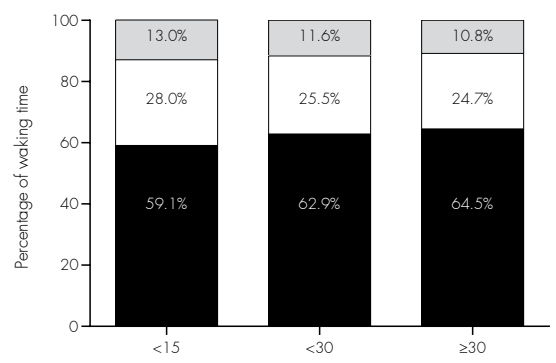

Albuminuria categories (mg/24h)

Figure 9.1: Percentage of waking time spent sedentary, standing, and physically active according to categories of eGFR crys (Panel A) and albuminuria (Panel B). Black bars indicate sitting/ lying; white bars indicate standing; grey bars indicate physical activity (stepping). Distribution of participants in the eGFR crcys $_{\text {categories: }} \geq 90 \mathrm{ml} / \mathrm{min} / 1.73 \mathrm{~m}^{2} \mathrm{n}=1,073 ;<90 \mathrm{ml} / \mathrm{min} / 1.73 \mathrm{~m}^{2}$ $n=1,077 ;<60 \mathrm{ml} / \mathrm{min} / 1.73 \mathrm{~m}^{2} \mathrm{n}=97$. Distribution of participants in the albuminuria categories: $<15 \mathrm{mg} / 24 \mathrm{hn}=1,804 ;<30 \mathrm{mg} / 24 \mathrm{~h} \mathrm{n}=246 ; \geq 30 \mathrm{mg} / 24 \mathrm{~h} \mathrm{n}=197$.

\section{Estimated glomerular filtration rate (eGFR)}

Table 9.2 presents the adjusted means of the physical activity and sedentary behaviour variables according to eGFR crys $_{\text {categories. Overall, participants with a lower eGFR }}$ crys had lower levels of physical activity (total, LPA, and HPA) after adjustment for confounders in model $1(P<0.05)$. Between the highest and lowest eGFR ${ }_{\text {crcys }}$ category, a difference of $14 \mathrm{~min} /$ day $(0.23 \mathrm{~h})$ was seen for total physical activity, $9 \mathrm{~min} /$ day $(0.15 \mathrm{~h})$ for LPA, and, after an additional adjustment for sedentary time, 4 min/day for HPA (model 3). The amount of sedentary time was larger in participants with lower eGFR crys, $_{\text {, }}$ at most 19 $\mathrm{min} /$ day $(0.32 \mathrm{~h})$ in model $2(\mathrm{P}=0.05)$. The number of sedentary breaks, prolonged sedentary bouts and average sedentary bout duration were similar in the categories after adjustment for confounders (model 3). 
Table 9.2: Adjusted means of physical activity and sedentary behaviour variables according to eGFR crcys $_{\text {categories }}$

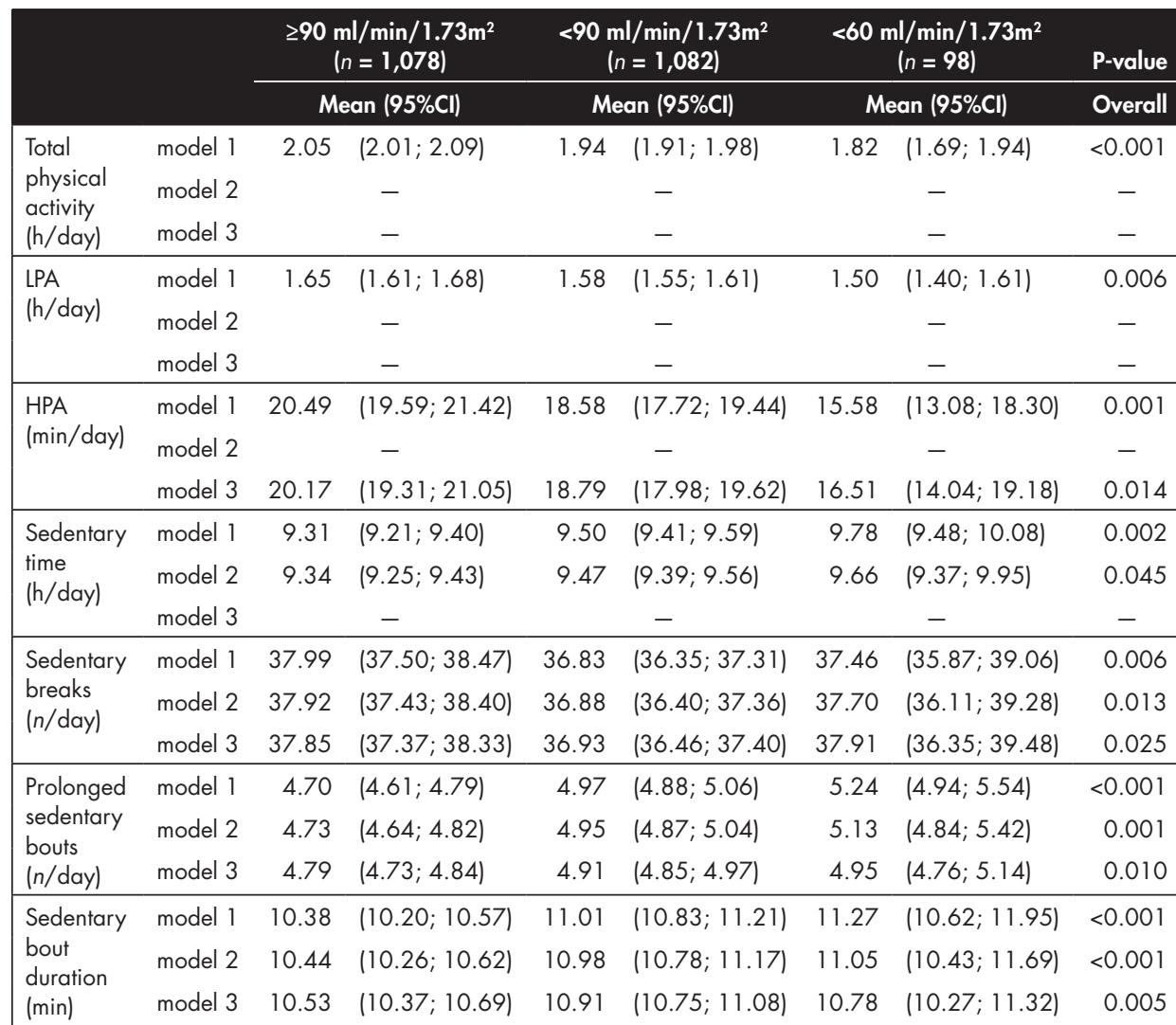

Adjusted means with 95\% confidence interval (95\% CI). LPA, lower intensity physical activity; HPA, higher intensity physical activity. In models 1 all variables were adjusted for age, sex, glucose metabolism status, waking time, educational level, smoking behaviour, alcohol consumption, energy intake, comorbid disease, mobility limitation, waking time; in models 2 the sedentary behaviour variables were additionally adjusted for HPA; in models 3, HPA and the sedentary behaviour pattern variables were additionally adjusted for sedentary time.

Table 9.3 presents the associations of the daily activity variables with eGFR amounts of total physical activity and LPA per day were statistically significantly associated

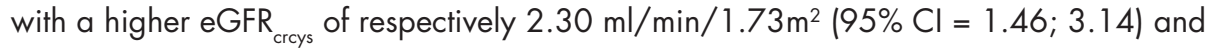
$2.10 \mathrm{ml} / \mathrm{min} / 1.73 \mathrm{~m}^{2}(1.08 ; 3.12)$, after adjustment for confounders (model 1). After additional adjustment for potential mediators (model 4), the associations were attenuated, but remained statistically significant. The amount of daily HPA was also statistically

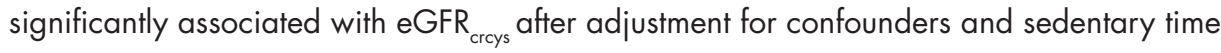


$(B=0.53 ;(0.21 ; 0.85))$, but after further adjustment for potential mediators in model 4 , the association was attenuated and no longer statistically significant. Larger amounts of daily sedentary time were statistically significantly associated with a lower eGFR of $0.71 \mathrm{ml} / \mathrm{min} / 1.73 \mathrm{~m}^{2}(-1.08 ;-0.35)$, after adjustment for confounders including HPA (model 2). After additional adjustment for potential mediators (model 4), the association was attenuated, but remained statistically significant. A larger number of sedentary breaks per day was associated with a higher eGFR crys $_{\text {in model } 2}(B=0.80 ;(0.12 ; 1.47))$, but after an additional adjustment for sedentary time in model 3 , the association became statistically nonsignificant. Having more daily prolonged sedentary bouts and having a longer average sedentary bout duration were both statistically significantly associated with a lower eGFR crys' even after adjustment for relevant confounders in model 3 and potential mediators in model $4\left(B_{\text {prolonged bouts }}=-0.57 ;(-1.14 ;-0.01) ; B_{\text {bout duration }}=-0.23 ;(-0.41 ;-0.04)\right)$.

Table 9.3: Associations of physical activity and sedentary behaviour variables with eGFR crys

\begin{tabular}{|c|c|c|c|c|c|c|c|c|}
\hline & \multirow{2}{*}{\multicolumn{2}{|c|}{$\frac{\text { Model } 1}{\text { B }(95 \% \mathrm{Cl})}$}} & \multirow{2}{*}{\multicolumn{2}{|c|}{$\frac{\text { Model } 2}{\text { B }(95 \% \mathrm{CI})}$}} & \multirow{2}{*}{\multicolumn{2}{|c|}{$\frac{\text { Model } 3}{\text { B }(95 \% \mathrm{Cl})}$}} & \multicolumn{2}{|r|}{ Model 4} \\
\hline & & & & & & & & $(95 \% \mathrm{Cl})$ \\
\hline $\begin{array}{l}\text { Total physical } \\
\text { activity (h/day) }\end{array}$ & 2.30 & $(1.46 ; 3.14)$ & & - & & - & 1.55 & $(0.69 ; 2.40)$ \\
\hline $\begin{array}{l}\text { LPA } \\
\text { (h/day) }\end{array}$ & 2.10 & $(1.08 ; 3.12)$ & & - & & - & 1.49 & $(0.47 ; 2.50)$ \\
\hline $\begin{array}{l}\text { HPA } \\
\text { (10 min/day) }\end{array}$ & 0.70 & $(0.39 ; 1.02)$ & & - & 0.53 & $(0.21 ; 0.85)$ & 0.31 & $(-0.02 ; 0.64)$ \\
\hline $\begin{array}{l}\text { Sedentary time } \\
\text { (h/day) }\end{array}$ & -0.88 & $(-1.23 ;-0.53)$ & -0.71 & $(-1.08 ;-0.35)$ & & - & -0.47 & $(-0.84 ;-0.10)$ \\
\hline $\begin{array}{l}\text { Sedentary } \\
\text { breaks (10/day) }\end{array}$ & 0.93 & $(0.26 ; 1.61)$ & 0.80 & $(0.12 ; 1.47)$ & 0.59 & $(-0.10 ; 1.27)$ & 0.51 & $(-0.17 ; 1.19)$ \\
\hline $\begin{array}{l}\text { Prolonged } \\
\text { sedentary } \\
\text { bouts (n/day) }\end{array}$ & -0.96 & $(-1.32 ;-0.61)$ & -0.82 & $(-1.19 ;-0.46)$ & -0.66 & $(-1.23 ;-0.09)$ & -0.57 & $(-1.14 ;-0.01)$ \\
\hline $\begin{array}{l}\text { Average } \\
\text { sedentary bout } \\
\text { duration (min) }\end{array}$ & -0.41 & $(-0.57 ;-0.26)$ & -0.35 & $(-0.51 ;-0.20)$ & -0.27 & $(-0.45-0.08)$ & -0.23 & $(-0.41 ;-0.04)$ \\
\hline
\end{tabular}

Regression results are presented as unstandardized coefficients (B) with $95 \%$ confidence intervals (95\% CI). Boldface indicates statistical significance (P < 0.05). LPA, lower intensity physical activity; HPA, higher intensity physical activity. In models 1 all variables were adjusted for age, sex, glucose metabolism status, waking time, educational level, smoking behaviour, alcohol consumption, energy intake, comorbid disease, mobility limitation, waking time; in models 2 the sedentary behaviour variables were additionally adjusted for HPA; in models 3, HPA and the sedentary behaviour pattern variables were additionally adjusted for sedentary time; in models 4 all variables were additionally adjusted for office systolic blood pressure, use of antihypertensive medication, waist circumference, total-to-HDL cholesterol ratio, triglycerides, use of lipid-modifying medication, prevalent cardiovascular disease. 


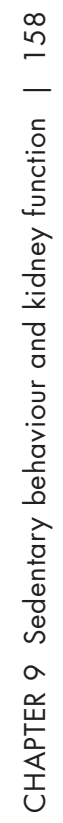

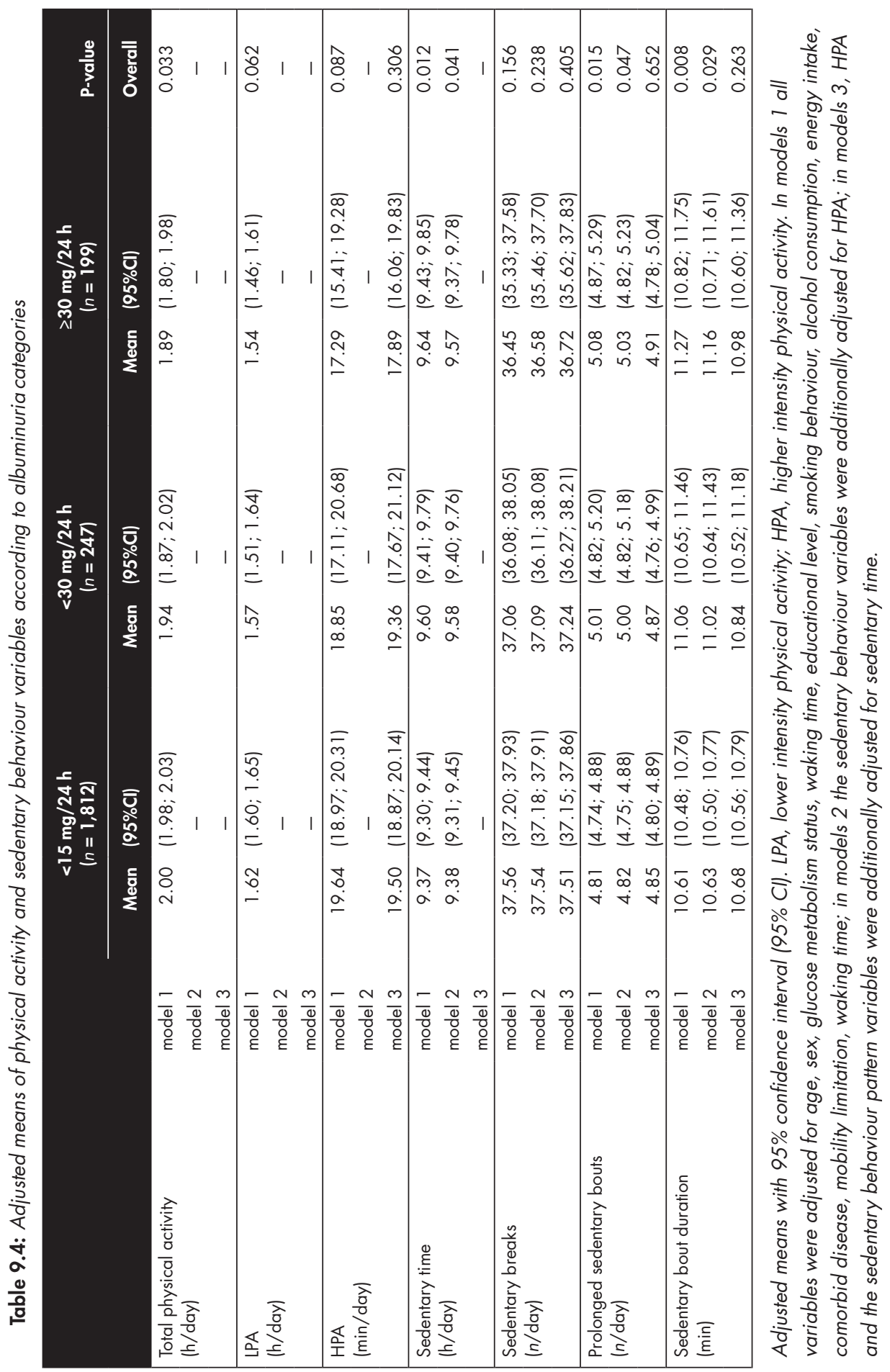




\section{Albuminuria}

Table 9.4 presents the adjusted means of the physical activity and sedentary behaviour variables according to albuminuria categories. Participants with a higher UAE had, after adjustment for confounders, lower levels of total physical activity $(7 \mathrm{~min} /$ day $(0.11 \mathrm{~h})$; $\mathrm{P}<0.05$; model 1), but only borderline statistically significant differences were seen for LPA or HPA. The amount of sedentary time was larger in participants with a higher UAE: on average $11 \mathrm{~min}(0.19 \mathrm{~h})$ more sedentary time per day $(P<0.05$; model 2$)$. The number of daily sedentary breaks was similar in the three categories and did not differ statistically significantly in any model. The differences in the number of prolonged sedentary bouts and average sedentary bout duration were, although statistically significant in models 1 and 2, less than 1 bout and 1 min respectively.

Table 9.5 presents the associations of the daily activity variables with albuminuria. The associations between total physical activity and LPA were non-linear and described using quartiles; therefore three odds ratios for each category are presented. Larger amounts of total physical activity were associated with lower odds ratios for higher UAE, although not all quartiles were statistically significant. After adjustment for potential mediators in model 4 , most odds ratios became statistically nonsignificant, although the odds ratios for an excretion rate of $<30 \mathrm{mg} / 24 \mathrm{~h}$ were similar to those in model 1 . The associations of LPA and HPA with albuminuria were, after adjustment for confounders (models 1 and 3), not statistically significant. In contrast, larger amounts of daily sedentary time were associated with a statistically significantly higher odds for a UAE of $<30 \mathrm{mg} / 24 \mathrm{~h}$ $\left(O R_{<30 \mathrm{mg} / 24 \mathrm{~h}}=1.11 ;(1.01 ; 1.22)\right)$ and with a borderline significantly higher odds for a $U A E$ of $\geq 30 \mathrm{mg} / 24 \mathrm{~h}\left(\mathrm{OR}_{\geq 30 \mathrm{mg} / 24 \mathrm{~h}}=1.10 ;(0.99 ; 1.22)\right)$ in model 2 . After adjustment for potential mediators in model 4 , the association with a UAE of $<30 \mathrm{mg} / 24 \mathrm{~h}$ was similar to the association in model 2 . The daily number of sedentary breaks was not significantly associated with albuminuria in any model. Having more daily prolonged sedentary bouts and having a longer average sedentary bout duration were associated with a higher odds for a UAE of $<30 \mathrm{mg} / 24 \mathrm{~h}$ and $\geq 30 \mathrm{mg} / 24 \mathrm{~h}$ in model 1 . These associations were no longer statistically significant after adjustment for HPA and sedentary time (models 2 and 3). 


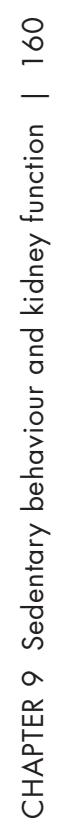

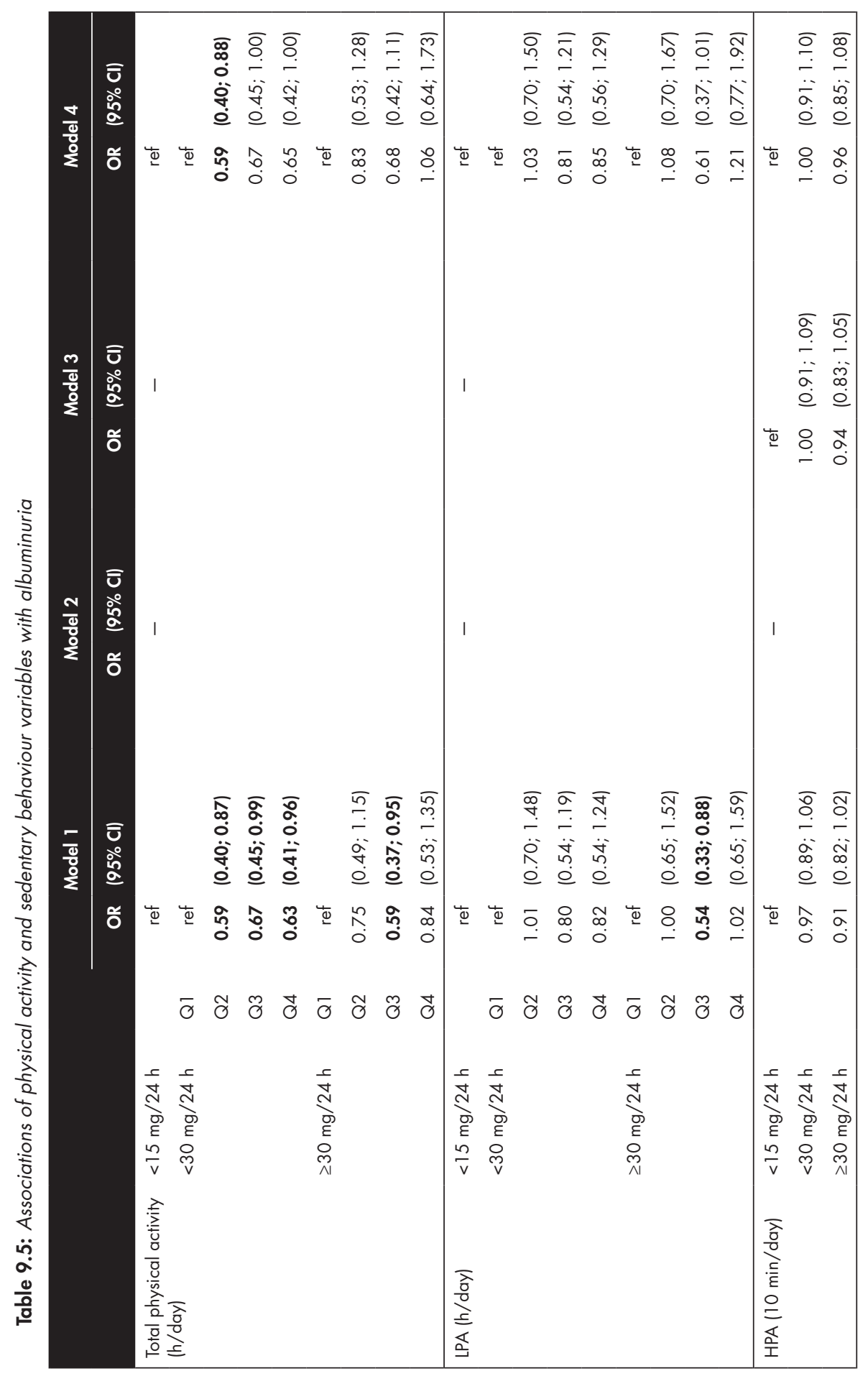




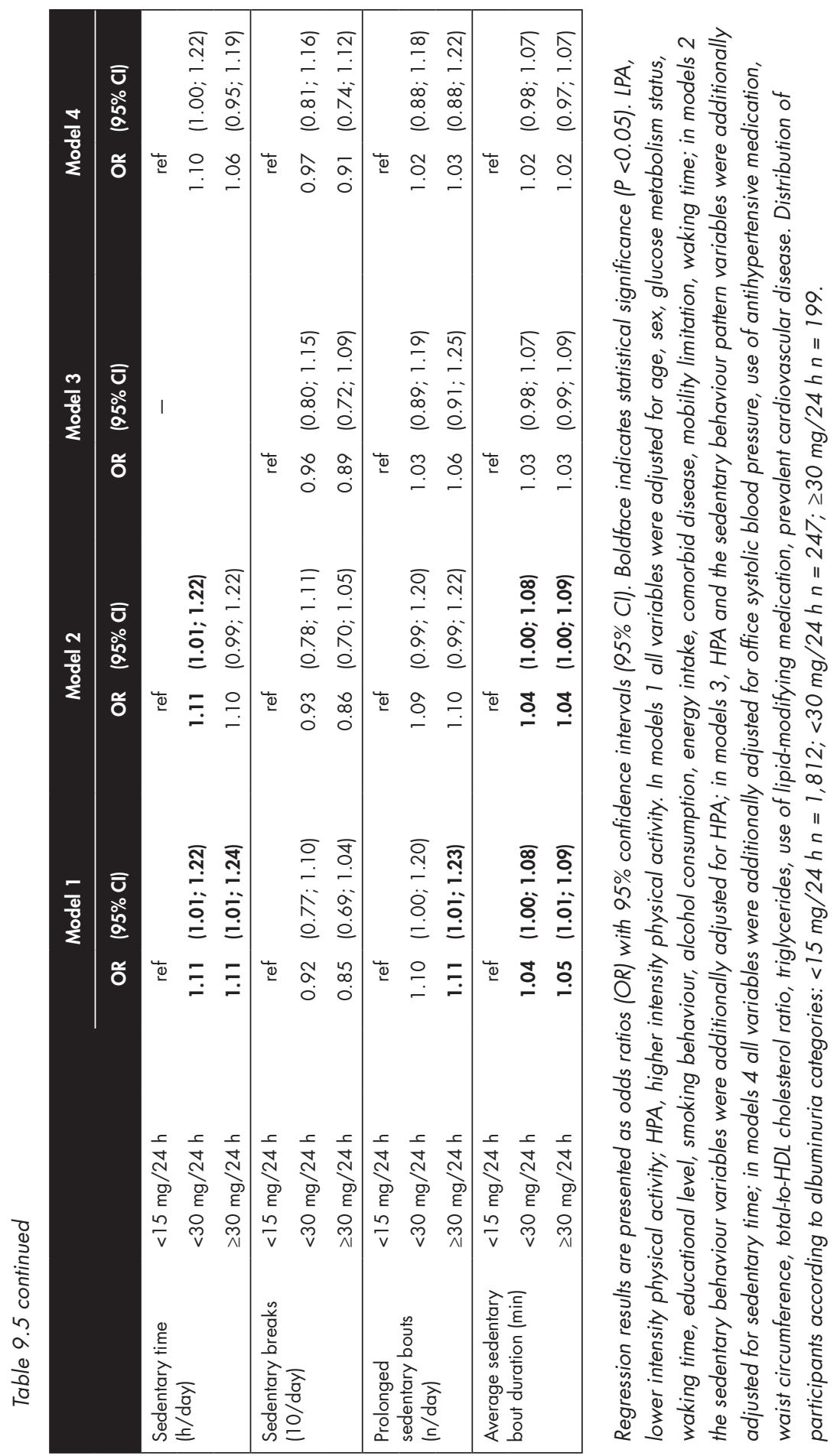




\section{Additional analyses}

In additional analyses, office systolic blood pressure was replaced with $24 \mathrm{~h}$ average ambulatory systolic blood pressure $(n=1,996)$. The effect sizes of the associations of all

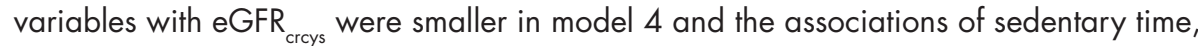
prolonged sedentary bouts and average sedentary bout duration with eGFR crcys $_{\text {became }}$ nonsignificant in model 4, possibly due to the loss of 262 participants with missing ambulatory systolic blood pressure data (data not tabulated). The effect sizes of the associations of all physical activity and sedentary behaviour variables with albuminuria were also attenuated, but the associations of total physical activity and sedentary time remained statistically significant. Further, results were similar when the analyses were repeated with replacement of antihypertensive medication with renin-angiotensin-aldosterone system inhibitors or when participants with a UAE of $>300 \mathrm{mg} / 24 \mathrm{~h}$ were excluded or when participants with $<4$ valid days ( $\geq 10 \mathrm{~h}$ of waking data) were excluded.

\section{DISCUSSION}

To our knowledge, this is the largest study in which posture-based accelerometry dato were used to measure total amount and patterns of physical activity and sedentary behaviour in order to examine associations of daily activity with kidney function. The results demonstrated that an extra hour of total physical activity (stepping time) or LPA was associated with an up to $2.30 \mathrm{ml} / \mathrm{min} / 1.73 \mathrm{~m}^{2}$ higher eGFR crcys after adjustment for confounders. Also, larger amounts of daily HPA (10min/day) were associated with a higher eGFR crcys $_{\text {of }} 0.53 \mathrm{ml} / \mathrm{min} / 1.73 \mathrm{~m}^{2}$. Further, each extra hour of sedentary time was associated with a lower eGFR crys $_{\text {of }} 0.71 \mathrm{ml} / \mathrm{min} / 1.73 \mathrm{~m}^{2}$ and also the pattern in which sedentary time was accumulated was associated with a lower eGFR crcys , independent of the amount of HPA and sedentary time. Given the average yearly $0.75-1 \mathrm{ml} / \mathrm{min} / 1.73 \mathrm{~m}^{2}$ age-related decline in GFR after the age of 40 years, ${ }^{35}$ these results may be clinically relevant.

The total amount of physical activity per day was also associated with albuminuria. Being more physically active resulted in lower odds for having higher UAE. The separate associations of LPA and HPA with albuminuria were not statistically significant. The small numbers of participants in the quartiles of LPA may have contributed to the nonsignificant associations. Alternatively, the amounts of LPA and HPA may have been insufficient to 
detect a significant association with albuminuria. Each extra hour of sedentary time was associated with a statistically significantly higher odds for having a higher UAE. Sedentary behaviour patterns were not statistically significantly associated with albuminuria after adjustment for confounders. From a pathophysiological perspective, albuminuria is hypothesized to be a biomarker of generalized endothelial dysfunction (i.e. endothelial dysfunction in the micro- and macrocirculation) and capillary rarefaction. ${ }^{36}$ Hence, our results suggest that a sedentary lifestyle may contribute to the development of generalized endothelial dysfunction and/or capillary rarefaction. This notion may be supported by previous studies which have shown effects of sedentariness on the endothelium. ${ }^{37,38}$

The associations of physical activity and sedentary behaviour with eGFR and albuminuria may be (partly) mediated by traditional risk factors such as T2DM, ${ }^{26,39-41}$ blood pressure, ${ }^{42}$ adiposity, ${ }^{43-47}$ and dyslipidaemia. ${ }^{43-46,48}$ Therefore, the adjustment for glucose metabolism status (to take into account the oversampling of participants with T2DM in The Maastricht Study) could have resulted in overadjustment bias. ${ }^{49}$ Analyses without adjustment for glucose metabolism status suggested that the associations with albuminuria may have been underestimated (data not shown). Further, the small changes of the regression coefficients of both eGFR $\mathrm{crcys}_{\text {s }}$ and albuminuria after adding potential mediators including waist circumference, blood pressure, lipid profile and prior CVD in model 4 suggested that these risk factors did not fully explain the reported associations. Therefore, physical activity and sedentary behaviour may be associated with eGFR crcys and albuminuria via other mechanisms such as low-grade inflammation, ${ }^{50}$ endothelial dysfunction, ${ }^{37,38,51}$ reduced activity of the renin-angiotensin system, ${ }^{52}$ reduced renal sympathetic nerve activity, ${ }^{51}$ and/or currently unknown effects. In addition, some factors in model 4 , such as adiposity, may have been confounding factors as well. So, true effect sizes of the associations of physical activity and sedentary behaviour with eGFR albuminuria were probably smaller than those reported in the partly adjusted models but larger than those in the fully adjusted model.

Taken together, our study provides new insights into the risk factors for reduced eGFR and albuminuria, which is important, given the increase in reduced eGFR and albuminuria and the associated burden of end-stage renal disease and CVD. Our results suggest that public health strategies to prevent a decline in eGFR and prevent the development of albuminuria should not only focus on increasing physical activity levels, but on reducing time spent sedentary as well. In addition, with regard to eGFR, further benefits may be obtained by altering the pattern of sedentary behaviour. 
Previously, studies have reported on associations of physical activity or sedentary behaviour with kidney function. Some studies did show associations of physical activity with eGFR $8,11,14$ or albuminuria, ${ }^{9-11,13}$ while others did not. ${ }^{12,15}$ Similar inconsistent results have been reported in studies on sedentary behaviour and eGFR ${ }^{11,16,18,19}$ or albuminuria. ${ }^{11,16,19}$ This inconsistency could have been due to the use of self-reporting measures for physical activity and sedentary behaviour, which easily could have been subject to recall and reporting bias. ${ }^{53}$ Only a few studies have used accelerometry data to examine associations of physical activity and sedentary behaviour with kidney function. In line with our results, it was reported previously that in individuals with reduced eGFR and/or albuminuria, smaller amounts of LPA and larger amounts of sedentary time were associated with a lower eGFR. ${ }^{20}$ In contrast, a longitudinal study conducted among individuals with T2DM reported that changes in MVPA and sedentary time were associated with changes in serum creatinine, but not with eGFR and UAE. ${ }^{21}$ These inconsistencies with our results may have been caused by differences in study design (cross-sectional vs. longitudinal) and study population, but may also be due to differences in the determination of physical activity and sedentary behaviour. We used the thigh-worn activPAL accelerometer, which classifies activity using data on posture in combination with acceleration, so our estimates of sedentary time were more accurate than those in other studies which were based solely on acceleration. ${ }^{54-56}$

Thus, the use of posture-based accelerometry data and the measurement of both total amount and patterns of daily activity were major strengths of our study. Another strength was the adjustment for an extensive series of potential confounders, including mutual adjustment for HPA and sedentary time, although residual confounding cannot be excluded. Some limitations should also be mentioned, of which the most important is the cross-sectional nature of our analyses. Therefore, we cannot make strong causal inferences, and reverse causality cannot be excluded. However, the previously reported association between changes of daily activity levels with changes in serum creatinine ${ }^{21}$ suggests that the direction of the association is from daily activity levels to kidney function in individuals with a mildly to moderately reduced eGFR. Nevertheless, longitudinal studies are needed to confirm this assumption. In addition, due to missing data, we had to exclude almost 1,200 participants. However, the excluded participants did not differ from our study sample with regard to demographic factors, accelerometry variables and kidney function (data not shown). Further, LPA and HPA were based on step frequency, which is less precise than using acceleration to determine intensity levels. However, it has been 
demonstrated that a step frequency of $>\sim 100$ steps $/ \mathrm{min}$ equals a MET score of $\geq 3.0^{31}$ and we used a cut-off point of $>110$ steps/min for HPA. Finally, our study population consisted of a relatively healthy population of primarily Caucasians from European descent with well-controlled individuals with T2DM. Therefore the results might not be representative for the general population of adults aged 40-75 years or other ethnicities.

To conclude, this large posture-based accelerometry study showed that both total physical activity and sedentary behaviour were associated with eGFR crcys $_{\text {s }}$ and albuminuria. Furthermore, the pattern in which sedentary time was accumulated may be of importance

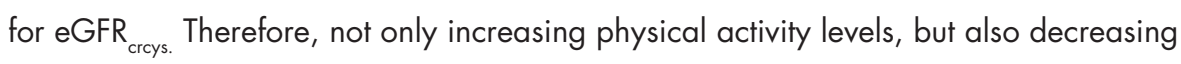
amount of sedentary time may be relevant targets in strategies aiming to prevent a reduced eGFR crys and albuminuria. However, future (longitudinal) accelerometry studies are needed to confirm our results and to further disentangle the associations of (patterns of) daily activity with kidney function. 


\section{REFERENCES}

1. National Kidney F. K/DOQI clinical practice guidelines for chronic kidney disease: Evaluation, classification, and stratification. Am J Kidney Dis. 2002;39(2 Suppl 1):S1-266.

2. Coresh J, Selvin E, Stevens LA, Manzi J, Kusek JW, Eggers $\mathrm{P}$, et al. Prevalence of chronic kidney disease in the United States. Jama. 2007;298(17):2038-47.

3. Chronic Kidney Disease Prognosis Consortium, Matsushita K, van der Velde M, Astor BC, Woodward M, Levey AS, et al. Association of estimated glomerular filtration rate and albuminuria with all-cause and cardiovascular mortality in general population cohorts: A collaborative meta-analysis. Lancet. 2010;375(9731):2073-81.

4. Ainsworth $B E$, Haskell WL, Whitt MC, Irwin ML, Swartz AM, Strath SJ, et al. Compendium of physical activities: An update of activity codes and MET intensities. Med Sci Sports Exerc. 2000;32(Suppl 9):S498-504.

5. Matthews CE, Chen KY, Freedson PS, Buchowski MS, Beech BM, Pate RR, et al. Amount of time spent in sedentary behaviors in the United States, 2003-2004. Am J Epidemiol. 2008;167(7):87581.

6. Davis MG, Fox KR, Hillsdon M, Sharp DJ, Coulson JC, Thompson JL. Objectively measured physical activity in a diverse sample of older urban UK adults. Med Sci Sports Exerc. $2011 ; 43(4): 647-54$.

7. Arnardottir NY, Koster A, Van Domelen DR, Brychta RJ, Caserotti P, Eiriksdottir G, et al. Objective measurements of daily physical activity patterns and sedentary behaviour in older adults: Age, Gene/Environment Susceptibility-Reykjavik Study. Age and ageing. 2013;42(2):222-9.

8. Finkelstein J, Joshi A, Hise MK. Association of physical activity and renal function in subjects with and without metabolic syndrome: A review of the Third National Health and Nutrition Examination Survey (NHANES III). Am J Kidney Dis. 2006;48(3):372-82.

9. Solbu MD, Kronborg J, Eriksen BO, Jenssen TG, Toft I. Cardiovascular risk-factors predict progression of urinary albumin-excretion in a general, non-diabetic population: A genderspecific follow-up study. Atherosclerosis. 2008;201 (2):398-406.

10. Robinson ES, Fisher ND, Forman JP, Curhan GC. Physical activity and albuminuria. Am J Epidemiol. 2010;171(5):515-21.

11. Bharakhada N, Yates T, Davies M, Wilmot EG, Edwardson C, Henson J, et al. Association of sitting time and physical activity with CKD: A cross-sectional study in family practices. Am J Kidney Dis. 2012;60(4):583-90.

12. Chang A, Van Horn L, Jacobs DR, Jr., Liu K, Muntner P, Newsome B, et al. Lifestyle-related factors, obesity, and incident microalbuminuria: The CARDIA (Coronary Artery Risk Development in Young Adults) study. Am J Kidney Dis. 2013;62(2):267-75.

13. Gutierrez-Repiso C, Rojo-Martinez G, Soriguer F, Garcia-Fuentes E, Vendrell J, Vazquez JA, et al. Factors affecting levels of urinary albumin excretion in the general population of Spain: The Di@betes study. Clin Sci. 2013;124(4):269-77.

14. Robinson-Cohen C, Littman AJ, Duncan GE, Weiss NS, Sachs MC, Ruzinski J, et al. Physical activity and change in estimated GFR among persons with CKD. J Am Soc Nephrol. 2014;25(2):399-406.

15. Herber-Gast $G C$, Hulsegge $G$, Hartman $L$, Verschuren WM, Stehouwer CD, Gansevoort RT, et al. Physical activity is not associated with estimated glomerular filtration rate among young and middle-aged adults: Results from the population-based longitudinal Doetinchem Study. PloS one. 2015;10(10):e0133864.

16. Lynch BM, White SL, Owen N, Healy GN, Chadban SJ, Atkins RC, et al. Television viewing time and risk of chronic kidney disease in adults: The AusDiab Study. Ann Behav Med. 2010;40(3):265-74.

17. Stengel B, Tarver-Carr ME, Powe NR, Eberhardt MS, Brancati FL. Lifestyle factors, obesity and the risk of chronic kidney disease. Epidemiology. 2003; 14(4):479-87.

18. Hallan $S$, de Mutsert R, Carlsen S, Dekker FW, Aasarod K, Holmen J. Obesity, smoking, and physical inactivity as risk factors for CKD: Are men more vulnerable? Am J Kidney Dis. 2006;47(3):396-405. 
19. White SL, Dunstan DW, Polkinghorne KR, Atkins RC, Cass A, Chadban SJ. Physical inactivity and chronic kidney disease in Australian adults: The AusDiab study. Nutr Metab Cardiovasc Dis. 2011;21 (2):104-12.

20. Hawkins MS, Sevick MA, Richardson CR, Fried LF, Arena VC, Kriska AM. Association between physical activity and kidney function: National Health and Nutrition Examination Survey. Med Sci Sports Exerc. $2011 ; 43(8)$ :1457-64.

21. Guo VY, Brage S, Ekelund U, Griffin SJ, Simmons RK. Objectively measured sedentary time, physical activity and kidney function in people with recently diagnosed type 2 diabetes: $A$ prospective cohort analysis. Diabet Med. 2015. Epub ahead of print.

22. Healy GN, Dunstan DW, Salmon J, Cerin E, Shaw JE, Zimmet PZ, et al. Breaks in sedentary time: Beneficial associations with metabolic risk. Diabetes care. 2008;31(4):661-6.

23. Healy GN, Winkler EA, Brakenridge $C L$, Reeves MM, Eakin EG. Accelerometer-derived sedentary and physical activity time in overweight/obese adults with type 2 diabetes: Cross-sectional associations with cardiometabolic biomarkers. PloS one. 2015;10(3):e0119140.

24. Bankoski A, Harris TB, McClain JJ, Brychta RJ, Caserotti P, Chen KY, et al. Sedentary activity associated with metabolic syndrome independent of physical activity. Diabetes care. $2011 ; 34(2): 497-503$.

25. Benatti FB, Ried-Larsen M. The effects of breaking up prolonged sitting time: A review of experimental studies. Med Sci Sports Exerc. 2015;47(10):2053-61.

26. Van der Berg JD, Stehouwer CD, Bosma $H$, van der Velde JH, Willems PJ, Savelberg HH, et al. Associations of total amount and patterns of sedentary behaviour with type 2 diabetes and the metabolic syndrome: The Maastricht Study. Diabetologia. 2016;59(4):709-18.

27. Schram MT, Sep SJ, van der Kallen CJ, Dagnelie PC, Koster A, Schaper N, et al. The Maastricht Study: An extensive phenotyping study on determinants of type 2 diabetes, its complications and its comorbidities. Eur J Epidemiol. 2014;29(6):439-51.

28. Inker LA, Schmid CH, Tighiouart $H$, Eckfeldt $\mathrm{JH}$, Feldman $\mathrm{HI}$, Greene T, et al. Estimating glomerular filtration rate from serum creatinine and cystatin C. N Engl J Med. 2012;367(1):209.

29. Hillege HL, Janssen WM, Bak AA, Diercks GF, Grobbee DE, Crijns HJ, et al. Microalbuminuria is common, also in a nondiabetic, nonhypertensive population, and an independent indicator of cardiovascular risk factors and cardiovascular morbidity. J Intern Med. 2001 ;249(6):519-26.

30. Van der Berg JD, Willems PJ, van der Velde JH, Savelberg HH, Schaper NC, Schram MT, et al. Identifying waking time in 24-h accelerometry data in adults using an automated algorithm. J Sports Sci. 2016;2:1-7.

31. Tudor-Locke C, Craig CL, Brown WJ, Clemes SA, De Cocker K, Giles-Corti B, et al. How many steps/day are enough? For adults. Int J Behav Nutr Phys Act. $2011 ; 8: 79$.

32. Tudor-Locke C, Rowe DA. Using cadence to study free-living ambulatory behaviour. Sports Med. 2012 May 1;42(5):381-98.

33. World Health Organization. Definition and Diagnosis of Diabetes Mellitus and Intermediate Hyperglycemia. World Health Organization 2006.

34. Altman DG. Practical statistics for medical research. London: Chapman and Hall/CRC; 1990. p. 143-5.

35. Levey AS, Coresh J. Chronic kidney disease. Lancet. 2012;379(9811):165-80.

36. Stehouwer CD, Smulders YM. Microalbuminuria and risk for cardiovascular disease: Analysis of potential mechanisms. J Am Soc Nephrol. 2006;17(8):2106-11.

37. Thosar SS, Bielko SL, Mather KJ, Johnston JD, Wallace JP. Effect of prolonged sitting and breaks in sitting time on endothelial function. Med Sci Sports Exerc. 2015;47(4):843-9.

38. Restaino RM, Holwerda SW, Credeur DP, Fadel PJ, Padilla J. Impact of prolonged sitting on lower and upper limb micro- and macrovascular dilator function. Exp Physiol. 2015;100(7):829-38.

39. Wilmot EG, Edwardson CL, Achana FA, Davies M, Gorely T, Gray W, et al. Sedentary time in adults and the association with diabetes, cardiovascular disease and death: Systematic review and meta-analysis. Diabetologia. 2012;55(11):2895-905. 
40. Biswas A, Oh PI, Faulkner GE, Bajaj RR, Silver MA, Mitchell MS, et al. Sedentary time and its association with risk for disease incidence, mortality, and hospitalization in adults: A systematic review and meta-analysis. Ann Intern Med. 2015;162(2):123-32.

41. Aune D, Norat T, Leitzmann M, Tonstad S, Vatten L. Physical activity and the risk of type 2 diabetes: A systematic review and dose-response meta-analysis. Eur J Epidemiol. 2015;30(7):529_ 42.

42. Huai $P$, Xun H, Reilly KH, Wang Y, Ma W, Xi $B$. Physical activity and risk of hypertension: A meta-analysis of prospective cohort studies. Hypertension. 2013;62(6): 1021-6.

43. Kelley GA, Kelley KS. Aerobic exercise and lipids and lipoproteins in men: A meta-analysis of randomized controlled trials. J Mens Health Gend. 2006;3(1):61-70.

44. Kelley GA, Kelley KS, Tran ZV. Aerobic exercise and lipids and lipoproteins in women: A metaanalysis of randomized controlled trials. J Womens Health (Larchmt). 2004;13(10):114864.

45. Cooper AR, Sebire S, Montgomery AA, Peters TJ, Sharp DJ, Jackson N, et al. Sedentary time, breaks in sedentary time and metabolic variables in people with newly diagnosed type 2 diabetes. Diabetologia. 2012;55(3):589-99.

46. Healy GN, Matthews CE, Dunstan DW, Winkler EA, Owen N. Sedentary time and cardiometabolic biomarkers in US adults: NHANES 2003-06. Eur Heart J. $2011 ; 32(5): 590-7$.

47. Healy GN, Wiindaele K, Dunstan DW, Shaw JE, Salmon J, Zimmet PZ, et al. Objectively measured sedentary time, physical activity, and metabolic risk: The Australian Diabetes, Obesity and Lifestyle Study (AusDiab). Diabetes care. 2008;31 (2):369-71.

48. Brocklebank LA, Falconer CL, Page AS, Perry R, Cooper AR. Accelerometer-measured sedentary time and cardiometabolic biomarkers: A systematic review. Prev Med. 2015;76:92-102.

49. Schisterman EF, Cole SR, Platt RW.

Overadjustment bias and unnecessary adjustment in epidemiologic studies. Epidemiology.

2009;20(4):488-95
50. Henson J, Yates T, Edwardson CL, Khunti K, Talbot D, Gray W, et al. Sedentary time and markers of chronic low-grade inflammation in a high risk population. PloS one. 2013;8(10):e78350.

51. Padilla J, Simmons GH, Bender SB, Arce-Esquive AA, Whyte JJ, Laughlin MH. Vascular effects of exercise: Endothelial adaptations beyond active muscle beds. Physiology. $2011 ; 26(3): 132-45$.

52. Goessler K, Polito M, Cornelissen VA. Effect of exercise training on the renin-angiotensinaldosterone system in healthy individuals: A systematic review and meta-analysis. Hypertens Res. 2016;39(3): 119-26.

53. Atkin AN, Gorely T, Clemes SA, Yates T, Edwardson C, Brage S, et al. Methods of measurement in epidemiology: Sedentary behaviour. Int J Epidemiol. 2012;41(5):146071

54. Godfrey A, Culhane KM, Lyons GM. Comparison of the performance of the activPAL Professional physical activity logger to a discrete accelerometer-based activity monitor. Med Eng Phys. $2007 ; 29(8): 930-4$

55. Kozey-Keadle S, Libertine A, Lyden K, Staudenmayer J, Freedson PS. Validation of wearable monitors for assessing sedentary behavior. Med Sci Sports Exerc. $2011 ; 43(8)$ :1561-7.

56. Edwardson $\mathrm{CL}$, Rowlands $\mathrm{AV}$, Bunnewell $\mathrm{S}$, Sanders J, Esliger DW, Gorely T, et al. Accuracy of posture allocation algorithms for thigh- and waist-worn accelerometers. Med Sci Sports Exerc. 2016. Epub ahead of print. 

Chapt

照 

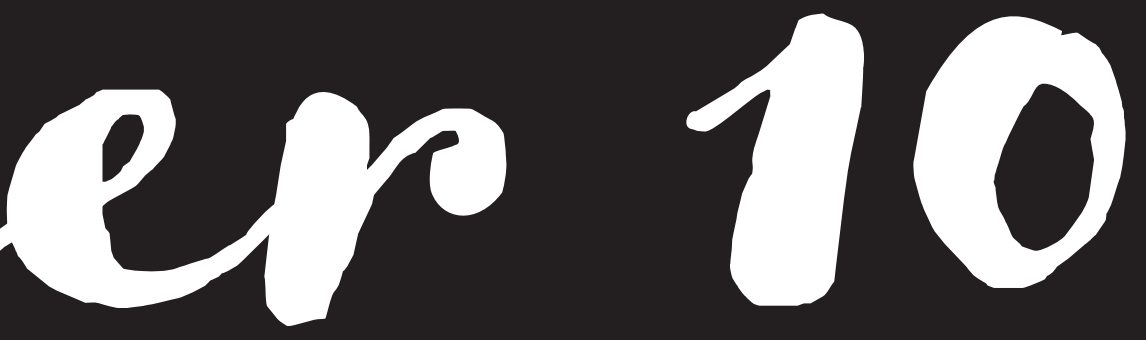

General discussion 
Type 2 diabetes mellitus (T2DM) is one of the most prevalent diseases worldwide. ${ }^{1}$

With its complications and comorbidities T2DM imposes an enormous burden on patients, healthcare systems and society, so the need for preventing T2DM is beyond dispute. For prevention strategies it is essential to identify targets that not only play a role in the development of T2DM and its complications and comorbidities, but are modifiable and amenable for intervention as well.

Sedentary behaviour, which is defined as any waking behaviour that is characterized by a low energy expenditure ( $\leq 1.5$ metabolic equivalents $(M E T s)$ ) while in a sitting or reclining position, ${ }^{2}$ for example using the computer or driving, may be such a target. Previous studies have demonstrated that large amounts of sedentary time are associated with several detrimental cardio-metabolic outcomes such as adiposity, ${ }^{3-6}$ dyslipidaemia, ${ }^{3-6}$ high blood glucose, ${ }^{3-8}$ the metabolic syndrome, ${ }^{3,4,6}$ and T2DM.9-11,8 However, most of these studies have used self-reporting measures, which could have been subject to report and recall bias. ${ }^{12-14}$ Apart from that, as not all sedentary behaviour is bad, the pattern in which sedentary time is accumulated should be taken into consideration, but studies on sedentary behaviour patterns are limited. To overcome these limitations this dissertation includes studies that have objectively measured several constructs of sedentary behaviour using an accelerometer, and have examined their associations with cardio-metabolic outcomes.

This chapter summarizes the main findings of the studies in this dissertation and discusses their methodological considerations. This is followed by an overview of the biological mechanisms that may explain the findings. Finally, implications for public health and clinical practice are provided as well as directions for future research.

\section{MAIN FINDINGS}

\section{Methods}

For researchers of studies that use an accelerometer with a 24 hour/day wear time protocol, a method to identify waking time is necessary in order to correctly determine the amount of sedentary time and physical activity accumulated during waking time. Therefore, we developed an algorithm for activPAL accelerometry data that automatically identifies wake and bed times on an individual level (i.e. different wake and bed times on each day for each participant). In chapter 2 we have demonstrated that this algorithm 
was highly associated with self-reported wake and bed times. So, using an accelerometer with a 24 hour/day wear time protocol was demonstrated to be feasible in large-scale epidemiological studies, such as The Maastricht Study.

In chapter 3 we have demonstrated, in an experimental setting, that a hip-worn tri-axial accelerometer records significant amounts of movement in the back-to-front and side-to-side directions during sedentary activities. So, tri-axial data can be used to distinguish individuals with more movement during sedentary activities from those with less movement. Since this movement may be relevant for health, we recommend the use of tri-axial accelerometry data in future sedentary behaviour studies.

\section{Determinants}

Previous studies have shown that large amounts of sedentary behaviour are associated with adverse cardio-metabolic outcomes. ${ }^{3,4,10,11,15-17}$ To limit the adverse effects of sedentary behaviour, it is necessary to study its determinants in order to identify individuals at increased risk for a (highly) sedentary lifestyle. In chapter 4 we have demonstrated that several factors measured in midlife were associated with larger amounts of sedentary time in old age. More specifically, marital status (not being married), level of education (primary), housing type (living in a duplex or an apartment), obesity, and heart disease were associated with up to 39 minutes more sedentary time per day.

\section{Cardio-metabolic health}

In the chapters 5-9 we examined several constructs of sedentary behaviour with cardio-metabolic health. In chapter 3 we have demonstrated that movement during sedentary activities can be measured using a hip-worn tri-axial accelerometer and we have recommended examining associations of this movement with health. In chapter 5 we used a hip-worn tri-axial accelerometer to measure movement during sedentary activities and introduced the term 'active sedentariness' to denote this movement. Subsequently, we have examined active sedentariness in relation to health and have demonstrated that larger amounts of active sedentariness were associated with improved cardio-metabolic outcomes including a lower body mass index, a smaller waist circumference, improved levels of HDL cholesterol and triglycerides, and a lower odds for the metabolic syndrome.

In chapter 6 we examined associations of theoretical reallocations of sedentary time to standing or stepping with cardio-metabolic outcomes, the metabolic syndrome, and T2DM. The results show that replacing 30 minutes of sedentary time with 30 minutes 
of standing or stepping was associated with a lower body mass index, a smaller waist circumference, and improved cholesterol, triglycerides, glucose and insulin levels. In addition, similar reallocations were favourably associated with the metabolic syndrome and T2DM.

Chapter 7 describes the associations of total amount and patterns of sedentary behaviour with the metabolic syndrome and T2DM. The results show that an extra hour of sedentary time was associated with a $39 \%$ increased odds for the metabolic syndrome and a $22 \%$ increased odds for T2DM. Sedentary behaviour patterns were only weakly associated with the metabolic syndrome.

In chapters 8 and 9 associations of total amount and patterns of physical activity and sedentary behaviour with risk factors for cardio-metabolic diseases were examined. These risk factors included stiffness of the aorta, which was determined by carotid-femoral pulse wave velocity (cfPWV), and kidney function, which was expressed by reduced estimated glomerular filtration rate (eGFR) and albuminuria. In chapter 8 , we found no significant associations of total amount and patterns of sedentary behaviour with cFPWV. In contrast, larger amounts of physical activity were associated with lower cFPWV. In chapter 9, adverse associations of sedentary behaviour with both eGFR and albuminuria were demonstrated. Patterns of sedentary behaviour were only associated with eGFR. In addition, physical activity was beneficially associated with eGFR as well as albuminuria.

\section{METHODOLOGY}

The results of the studies in this dissertation need to be discussed with due consideration of methodological limitations of the studies. These relate to the measurement of sedentary behaviour, the study design, and the statistical analyses of the studies.

\section{Measurement}

In all studies of this dissertation accelerometry was used to measure sedentary behaviour. As mentioned in chapter 1, accelerometers measure the frequency, duration and intensity of activity, so objective data on the amount of daily activity is provided. ${ }^{12,18}$ Consequently, accelerometry data overcomes important limitations of self-reported data such as report and recall bias. ${ }^{12-14}$ Nevertheless, issues that arise when using accelerometry relate to the identification of sedentary behaviour and its patterns, the reliability of estimations, and missing information on type and context of activity. 
Identification of sedentary behaviour and its patterns In the studies of chapters 6-9 the activPAL physical activity monitor was used. Since this device classifies activity based on posture-based data into sitting/lying, standing and stepping, accurate estimations of the amount of sedentary time were obtained. 14,19-21 However, in these studies acceleration data was not available, so some (high intensity) activities such as weight lifting may have been misclassified as sedentary time. ${ }^{14,21}$ In addition, the lack of acceleration data hampered identification of light, moderate and vigorous activity. As an alternative we used step frequency to distinguish higher and lower intensity physical activity. This method is less precise than using acceleration data to determine intensity of physical activity. However, it has been demonstrated that a step frequency of $>\sim 100$ steps/minute equals a MET score of $\geq 3.0,{ }^{22}$ which has been classified as moderate physical activity. ${ }^{23}$ We used a cut-off point of $>110$ steps/minute to identify higher intensity physical activity.

In the studies of chapters 7-9 we used sedentary breaks, prolonged sedentary bouts (accumulated in a consecutive period of 30 minutes), and average sedentary bout duration as constructs to quantify sedentary behaviour patterns. Due to the relatively recent interest in patterns of sedentary behaviour, a variety of definitions have been used for its constructs. For example, definitions of a sedentary break vary in duration (e.g. either any interruption or an interruption of at least 1 minute) and in the amount of sedentary time which precedes the break (e.g. either any duration or a duration of at least 1 minute). To overcome this issue to some extent, we have conducted additional analyses in our studies using sedentary breaks of any duration and sedentary breaks with a duration of at least 1 minute, which resulted in similar findings. In addition to inconsistent definitions, the determination of sedentary breaks differs between methods using posture data (transition in posture) and methods using acceleration data (exceeding $100 \mathrm{cpm}$ ). In our studies we have used posture data to determine breaks and this method has shown to be highly accurate. ${ }^{24}$ However, in other studies acceleration data have been used to determine sedentary breaks, although it has been shown that this method overestimates the number of breaks due to misclassification of standing time as sedentary time. ${ }^{24}$ The use of these different methods resulted in inconsistent findings on associations between sedentary breaks and several health outcomes. ${ }^{25-30}$ Furthermore, the meaning of sedentary breaks remains uncertain, as it is unclear what a sedentary break should reflect and what it does reflect, for example information on the frequency of sedentary bouts or on prolonged sedentary time. Similar issues are seen for prolonged sedentary bouts and average 
sedentary bout duration; associations with health outcomes have been inconsistent and uncertainties about their meaning exist. Clearly, fundamental work is needed to standardize and validate constructs of sedentary behaviour patterns. Apart from that, in our studies several constructs of sedentary behaviour patterns were not (or only weakly) associated with health outcomes. Therefore, the relevance of sedentary behaviour patterns for health needs further examination.

In the studies of chapters 4 and 5 the Actigraph accelerometer was used. This device provided tri-axial acceleration data, so a comprehensive measurement of activity was obtained. To identify sedentary time, a cut-off point of $<100$ counts per minute $(\mathrm{cpm})^{31}$ in the vertical axis was used. Although this cut-off point has been widely used and has been associated with health outcomes consistently, 4,11,16,17 the evidence on its validity is limited. Furthermore, we have demonstrated in chapter 2 that $\geq 100 \mathrm{cpm}$ in the vertical axis could be recorded while being in a sedentary position. So in chapters 4 and 5 , sedentary time may have been misclassified as physical activity and activities in an upright position may have been misclassified as sedentary time when counts were below 100 per minute.

\section{Reliability of estimations}

The reliability of the estimations of both sedentary behaviour and physical activity depends on the wear time of the accelerometer. ${ }^{14,32}$ In contrast to the activPAL accelerometer, the Actigraph accelerometer is usually worn during waking hours only and the device is removed during water-based activities. ${ }^{33}$ Therefore, not all daily activity will be assessed when a wear time protocol such as that of the Actigraph accelerometer is used. In order to obtain accurate estimations of daily activity levels, an accelerometer should be worn a considerable amount of the day. However, accelerometer wear time varies highly among participants; some provide 18 hours of data, while others provide only 4 . Wear time guidelines are currently not available, but a minimum wear time of 10 hours per day has been widely used. However, studies have shown that a wear time criterion of at least 10 hours per day resulted in an underestimation of daily sedentary time compared to a criterion of at least 14 hours per day. ${ }^{14,34,35}$ Two studies of this dissertation (chapters 4 and 5) have used the Actigraph accelerometer and a wear time criterion of at least 10 hours per day, thus the amount of sedentary time in these studies may have been underestimated. Four studies (chapters 6-9) have used the activPAL accelerometer, which had to be worn 24 hour per day. When participants removed the device on the final wear day, data was only included in analyses when the device had been worn at least 
14 hours after wake time. Consequently, in these studies accurate estimations of the amount of sedentary time were obtained.

For reliable estimations not only the wear time hours per day are important, the number of wear days is important as well. It has been shown that accelerometers should be worn at least 4 days to reliably estimate sedentary behaviour and at least 7 days for moderate to vigorous physical activity. ${ }^{32,36} \mathrm{~A}$ minimum number of wear days may overcome adaptations in activity levels due to awareness of participants of wearing an accelerometer, and may balance out irregularities in activity levels. Therefore, most studies, including those in this dissertation, have used a wear time protocol of 7 or 8 consecutive days. Compliance to this protocol was high in our studies, since the accelerometer was worn on average 6 days in both the cohorts of the AGES-Reykjavik Study and The Maastricht Study.

\section{Missing information}

The representation of sedentary behaviour using constructs such as duration, frequency and patterns as provided by accelerometers, grants valuable information about this behaviour. However, accelerometers do not provide information on other relevant constructs including the type of behaviour (e.g. watching TV, doing desk work or driving) and the social (individual or in groups) or environmental (leisure, work or transportation) context in which it occurs. ${ }^{12-14}$ The studies in this dissertation do therefore not provide this information. To determine whether detrimental health effects were due to sedentary behaviour itself, or to clustered other behaviours (e.g. snacking), or both, information on the type of behaviour and context is warranted. Therefore, subjective measures that provide insight in constructs such as type of behaviour and context should be added to objective sedentary behaviour data in future accelerometry studies.

\section{Study design}

In the studies of chapters 6-9 we used data of The Maastricht Study. ${ }^{37}$ Two main advantages of the study design of The Maastricht Study were its large study population and its extensive phenotyping approach, which enabled us to examine sedentary behaviour in relation to cardio-metabolic outcomes such as body mass index, lipids and blood pressure, the metabolic syndrome, T2DM, aortic stiffness and kidney function. Unfortunately, only data of the baseline survey were available, so our analyses were cross-sectional in nature. Therefore, (strong) causal relationships could not be determined 
and reverse causality could not be excluded. In addition, associations between sedentary behaviour and health might even be bidirectional. For example, a large amount of sedentary time may result in obesity, but being obese may also result in more sedentary time. For these reasons, conclusions about the direction of the associations have been made with caution.

In the studies of chapters 4 and 5 data of the AGES-Reykjavik Study ${ }^{38}$ were used. Although longitudinal data of several measures were available, longitudinal accelerometry data were not collected. Therefore, exposure and outcome variables in these studies were measured once, which has hampered drawing conclusions about the direction of the associations in these studies as well.

To overcome this limitation, longitudinal studies in which both exposure and outcome variables are repeatedly measured over time are needed. To date, no accelerometry studies have reported on longitudinal associations of sedentary behaviour with health, but longitudinal accelerometry data will probably become available in the near future.

\section{Statistical analysis}

The observational studies in this dissertation aimed to examine and quantify associations between sedentary behaviour and cardio-metabolic health. These associations cannot be examined by simply comparing the outcome variable (cardio-metabolic health) in groups with a different exposure variable (sedentary behaviour), because a variety of factors (the so-called confounders) may interact with both variables, which leads to biased results. To limit the possibility of biased results, the reported associations in this dissertation were adjusted for potential confounders. Although the potential confounders for each association differed to a certain extent, a common confounder in all associations was physical activity. To examine whether sedentary behaviour is associated with health independent of the amount of higher intensity physical activity, it is important to adjust the analyses for this factor. Therefore, all analyses in this dissertation were adjusted for higher intensity physical activity. The (statistically significant) results show that sedentary behaviour was independently associated with several health outcomes, which suggests that sedentary behaviour is a distinct type of behaviour with its own physiology that differs from a lack of higher intensity physical activity. Of note, in our analyses we did not adjust for light intensity physical activity, such as casual walking and household work (>1.5-<3.0 METs). ${ }^{23}$ Sedentary behaviour and light physical activity have shown to be highly correlated, 14,39,40 so due to issues of collinearity, adjustment for light physical activity in sedentary behaviour 
analysis is not possible. Because of the high correlation of sedentary behaviour and light physical activity, insight in the interaction between these types of behaviour in relation to health is of interest and this can be provided by experimental studies on effects of reallocating sedentary behaviour to light physical activity.

Further, although the datasets of the AGES-Reykjavik Study and The Maastricht Study included extensive data of the participants, which enabled us to adjust for a series of potential confounders, it was impossible to exclude residual confounding.

In addition, some potential confounding factors could also be a mediating factor, i.e. a factor that lies in the pathway between exposure and outcome measure. Adjustment for mediating factors leads to an underestimation of the association; the analyses are subject to overadjustment. ${ }^{41}$ In complex and multifactorial networks of conditions and diseases, such as cardio-metabolic health, it is difficult to determine whether a factor acts as a confounder, or as a mediator, or both. Therefore, our adjustment for a series of potential confounders to limit confounding bias may have introduced overadjustment bias. Nevertheless, to increase the credibility of the observed associations, adjustment for potential confounders was warranted, but the possibility of overadjustment should be kept in mind when interpreting the results.

\section{BIOLOGICAL MECHANISMS}

Several observational studies have demonstrated unfavourable associations between large amounts of sedentary time and cardio-metabolic health. 4,11,16,17 In addition, effects of reducing sedentary time by (inevitably) replacing it with non-sedentary time during waking hours have been examined in recent observational and experimental studies. Theoretical reallocations of sedentary time to light or high intensity physical activity, as examined in chapter 6 and other observational studies, were associated with favourable outcomes of body mass index, ${ }^{42-45}$ waist circumference, ${ }^{42,44-47}$ levels of cholesterol, triglyceride and glucose, ${ }^{43,44,46-48}$ the metabolic syndrome ${ }^{47}$ and T2DM. In experimental studies, the replacement of sedentary time varied from two minutes of light intensity physical activity to one hour of vigorous activity. A review showed that, overall, reducing sedentary time had a positive effect on metabolic outcomes including (postprandial responses of) glucose, insulin, HDL cholesterol, LDL cholesterol, triglycerides, and blood pressure. ${ }^{49}$ Taken together, a large body of evidence consistently links sedentary behaviour to adverse 
health outcomes. However, due to the relatively recent interest in sedentary behaviour as a risk factor for health, mechanisms that could explain how sedentary behaviour affects health are largely unknown. Bed rest studies have been used as a model to examine harmful effects of inactivity. Although these models do not accurately reflect daily patterns of sedentary behaviour, the studies do provide leads regarding physiological mechanisms of inactivity. A possible mechanism may be a reduction of lipoprotein lipase (LPL) due to inactivity of muscle cells, which has been seen in animal studies. ${ }^{50-52}$ Since LPL is an essential enzyme that contributes to the metabolism and transport of lipids, it can be hypothesized that a change in activity of this enzyme has a variety of effects on metabolism. ${ }^{50,51}$ Also reductions in lipid phosphate phosphatase-1 (LPP1) and decreasing adenosine monophosphate-activate protein kinase (AMPK) activity due to inactive muscle cells may be underlying mechanisms as both are involved in glucose metabolism. ${ }^{51,53}$ Other possible mechanisms may be changes in vascular function due to the absence of muscular contractions and increased blood flow. For example, it has been suggested that sedentary behaviour causes low mean shear stress which may affect endothelial function. ${ }^{54}$ In addition, sedentary behaviour may influence activity of the renin-angiotensin system, which regulates blood pressure and extracellular fluid. ${ }^{55}$ Lastly, it has been suggested that low-grade inflammation is a pathway through which sedentary behaviour could unfavourably affect health. ${ }^{56,57}$ Clearly, physiological studies are warranted to unravel the mechanisms and pathways through which sedentary behaviour affects health.

\section{IMPLICATIONS FOR PUBLIC HEALTH AND CLINICAL PRACTICE}

The studies in this dissertation have, in line with others, consistently shown that large amounts of sedentary time are associated with several adverse health outcomes. Still some associations were small and due to the aforementioned limitations of our studies, true effect sizes could not be determined. However, experimental studies support the hypothesis of a relation between sedentary behaviour and adverse health outcomes. ${ }^{49}$ Furthermore, even if true effect sizes would be small, the potential impact on population level may still be relevant as sedentary behaviour is highly prevalent on both an inter- and intra-individual level: the majority of individuals has been shown to spend on average more than half of the waking day in sedentary positions. ${ }^{58-61}$ Therefore, recommendations on reducing daily 
sedentary time to prevent its adverse effects on health are needed.

In some countries, guidelines aiming to reduce sedentary time have already been developed by public health organisations and scientific institutions. ${ }^{62-64}$ However, reducing sedentary time during waking time inevitably results in more non-sedentary time. So, the health effects of reducing sedentary time may depend on the amount of time that is replaced and on the activity with which it is replaced. So, specific recommendations on the amount of time and the type of activity with which sedentary time should be replaced are required. We demonstrated that, theoretically, replacement of even small amounts of sedentary time (i.e. 30 minutes) with non-sedentary time was associated with beneficial metabolic outcomes, the metabolic syndrome and T2DM (chapter 6). Future experimental and dose-response studies could use this information to study the effects of replacing sedentary time with non-sedentary time. Subsequently, effective guidelines on reducing sedentary behaviour could be developed.

Further, as sedentary time has been shown to be associated with several risk factors for T2DM and T2DM itself (chapter 7), consideration should be given to including strategies to reduce the amount of sedentary behaviour in diabetes prevention programs. Of note, prevention programs as well as guidelines on sedentary behaviour should be an addition to those of physical activity, as undoubtedly physical activity is an important factor in remaining healthy and preventing disease. ${ }^{65-68}$ Yet, sedentary behaviour guidelines could be of importance, particularly for individuals that cannot participate in (high intensity) physical activity, such as (frail) older adults and individuals with chronic diseases.

\section{DIRECTIONS FOR FUTURE RESEARCH}

As mentioned earlier, the field of sedentary behaviour research is relatively young. We were the first to use the activPAL accelerometer in a large study population, and the first who examined associations of objectively measured sedentary behaviour and its patterns with T2DM, cfPWV, eGFR and albuminuria. Although a new research field such as that of sedentary behaviour provides a plethora of research opportunities, it requires fundamental work in terms of validation and standardization.

To move the field of sedentary behaviour research forward, it is essential to validate and standardize constructs of sedentary behaviour, in particular those reflecting 
sedentary behaviour patterns. For example, standardization of a sedentary break (what should a sedentary break reflect and what measure should be used) increases comparability between studies and advances knowledge about its effect on health. In addition, comparability between studies is currently hampered due to the use of different accelerometers (e.g. activPAL or Actigraph) and different wear locations (e.g. thigh, hip, waist or wrist). Therefore, consideration should be given to standardization of the measurement method as well.

Another important aspect to improve sedentary behaviour research is combining different objective measurement methods, i.e. combining posture- and acceleration based data. Together these data provide even more accurate assessment of the total activity spectrum ranging from sedentary behaviour to high intensity physical activity. In addition, subjective data (type of behaviour, context) should be collected parallel to objective data (frequency, duration, intensity). This information helps to understand the nature of sedentary behaviour better and can be used to develop specific strategies aiming to prevent prolonged sedentary time, for example in the work environment or leisure domain.

To advance the biological understanding of sedentary behaviour physiological studies examining underlying mechanisms are needed. In addition, dose-response and longitudinal studies should be conducted to provide insight in harmful amounts of sedentary time and causality. Further, experimental and intervention studies examining effects of reducing sedentary time are warranted to gain information on frequency, duration and type of activity with which sedentary time should be replaced. Finally, feasibility studies are needed to examine whether and how reductions in sedentary behaviour can be achieved. 


\section{REFERENCES}

1. International Diabetes Federation. IDF Diabetes Atlas. 2015.

2. Sedentary Behaviour Research Network. Standardized use of the terms "sedentary" and "sedentary behaviours." Appl Physiol Nutr Metab. 2012;37:540-2.

3. Ford ES, Kohl HW, Mokdad AH, Ajani UA Sedentary behavior, physical activity, and the metabolic syndrome among U.S. adults. Obes Res. 2005; 13(3):608-14

4. Edwardson CL, Gorely T, Davies M, Gray L, Khunti K, Wilmot EG, et al. Association of sedentary behaviour with metabolic syndrome: $A$ meta-analysis. PLoS One. 2012;7(4):e34916.

5. Healy GN, Dunstan DW, Salmon J, Shaw JE, Zimmet PZ, Owen N. Television time and continuous metabolic risk in physically active adults. Med Sci Sports Exerc. 2008;40(4):639_ 45 .

6. Dunstan DW, Salmon J, Owen N, Armstrong T, Zimmet PZ, Welborn TA, et al. Associations of TV viewing and physical activity with the metabolic syndrome in Australian adults. Diabetologia. 2005;48(1 1):2254-61.

7. Dunstan DW, Salmon J, Healy GN, Shaw JE, Jolley D, Zimmet PZ, et al. Association of television viewing with fasting and $2-h$ postchallenge plasma glucose levels in adults without diagnosed diabetes. Diabetes Care. 2007;30(3):516-22.

8. Dunstan DW, Salmon J, Owen N, Armstrong T, Zimmet PZ, Welborn, Timothy A Camerson AJ, et al. Physical activity and television viewing in relation torisk of undiagnosed abnormal glucose metabolism in adults. Diabetes Care. 2004;27(1 1):2603-9.

9. Hu FB, Leitzmann MF, Stampfer M, Colditz GA, Willett WC, Rimm EB. Physical activity and television watching in relation to risk for type 2 diabetes mellitus in men. Arch Intern Med. $2001 ; 161: 1542-8$.

10. Grøntved A. Television viewing and risk of type 2 diabetes, cardiovascular disease, and all-cause mortality. JAMA. $2011 ; 305(23)$ :2448-55.
11. Wilmot EG, Edwardson CL, Achana FA, Davies M, Gorely T, Gray L, et al. Sedentary time in adults and the association with diabetes, cardiovascular disease and death: Systematic review and meta-analysis. Diabetologia. 2012;55(1 1):2895-905.

12. Atkin AJ, Gorely T, Clemes SA, Yates T, Edwardson C, Brage S, et al. Methods of measurement in epidemiology: Sedentary behaviour. Int J Epidemiol. 2012;41 (5):146071 .

13. Healy GN, Clark BK, Winkler EAH, Gardiner PA Brown WJ, Matthews CE. Measurement of adults' sedentary time in population-based studies. Am J Prev Med. $2011 ; 41$ (2):216-27.

14. Kang M, Rowe DA. Issues and challenges in sedentary behavior measurement. Meas Phys Educ Exerc Sci. 2015;19(3):105-15.

15. Ford ES, Caspersen CJ. Sedentary behaviour and cardiovascular disease: A review of prospective studies. Int J Epidemiol. 2012;41(5):1338-53.

16. Brocklebank LA, Falconer CL, Page AS, Perry R Cooper AR. Accelerometer-measured sedentary time and cardiometabolic biomarkers: A systematic review. Prev Med. 2015;76:92-102.

17. Biswas A, Oh PI, Faulkner GE, Bajaj RR, Silver MA, Mitchell MS, et al. Sedentary time and its association with risk for disease incidence, mortality, and hospitalization in adults. Ann Intern Med. 2015;162(10):123-32.

18. Butte NF, Ekelund U, Westerterp KR. Assessing physical activity using wearable monitors: Measures of physical activity. Med Sci Sports Exerc. 2012;44(1 Suppl 1):S5-12.

19. Kozey-Keadle S, Libertine A, Lyden K, Staudenmayer J, Freedson PS. Validation of wearable monitors for assessing sedentary behavior. Med Sci Sport Exerc. $2011 ; 43(8): 1561-7$.

20. Godfrey A, Culhane KM, Lyons GM. Comparison of the performance of the activPAL ${ }^{T M}$ Professional physical activity logger to a discrete accelerometer-based activity monitor. Med Eng Phys. 2007;29(8):930-4.

21. Janssen X, Cliff DP. Issues related to measuring and interpreting objectively measured sedentary behavior data. Meas Phys Educ Exerc Sci. 2015;19(3): 116-24. 
22. Tudor-Locke C, Craig CL, Brown WJ, Clemes SA, De Cocker K, Giles-Corti B, et al. How many steps/day are enough? For adults. Int J Behav Nutr Phys Act. $2011 ; 8(1): 79$.

23. Ainsworth BE, Haskell WL, Whitt MC, Irwin ML, Swartz ANNM, Strath SJ, et al. Compendium of physical activities: An update of activity codes and MET intensities. Med Sci Sports Exerc. 2000;32(Suppl 9):S498-504.

24. Lyden K, Kozey Keadle SL, Staudenmayer JW, Freedson PS. Validity of two wearable monitors to estimate breaks from sedentary time. Med Sci Sports Exerc. 2012;44(1 1):2243-52.

25. Healy G, Dunstan D, Salmon J, Cerin E, Shaw J, Zimmet $P$, et al. Breaks in sedentary time. Diabetes Care. 2008;31(4):661-3.

26. Bankoski A, Harris TB, McClain JJ, Brychta RJ, Caserotti P, Chen KY, et al. Sedentary activity associated with metabolic syndrome independent of physical activity. Diabetes Care. $2011 ; 34(2): 497-503$.

27. Healy GN, Matthews CE, Dunstan DW, Winkler EAH, Owen N. Sedentary time and cardiometabolic biomarkers in US adults: NHANES 2003-06. Eur Heart J. 2011 ;32(5):590-7.

28. Cooper AR, Sebire S, Montgomery AA, Peters TJ, Sharp DJ, Jackson N, et al. Sedentary time, breaks in sedentary time and metabolic variables in people with newly diagnosed type 2 diabetes. Diabetologia. 2012;55(3):589-99.

29. Gennuso KP, Gangnon RE, Thraen-Borowski KM Colbert LH. Dose-response relationships between sedentary behaviour and the metabolic syndrome and its components. Diabetologia. 2014;58:485-92.

30. García-Hermoso A, Martínez-Vizcaíno V, Recio-Rodríguez Jl, Sánchez-López M, GómezMarcos MÁ, García-Ortiz L. Sedentary behaviour patterns and carotid intima-media thickness in Spanish healthy adult population. Atherosclerosis. 2015;239(2):571-6.

31. Freedson PS, Melanson E, Sirard J. Calibration of the computer science and applications, inc. accelerometer. Med Sci Sport Exerc. 1998;30(5):777-81.

32. Edwardson $\mathrm{CL}$, Winkler EAH, Bodicoat $\mathrm{DH}$, Yates T, Davies M, Dunstan DW, et al. Considerations when using the activPAL monitor in field based research with adult populations. J Sport Heal Sci. 2016. Epub ahead of print.
33. Tudor-Locke C, Camhi SM, Troiano RP. A catalog of rules, variables, and definitions applied to accelerometer data in the National Health and Nutrition Examination Survey, 2003-2006. Prev Chronic Dis. 2012;9:E113.

34. Herrmann SD, Barreira TV, Kang $M$, Ainsworth BE. Impact of accelerometer wear time on physical activity data: A NHANES semisimulation data approach. Br J Sports Med. 2014;48(3):278-82.

35. Herrmann SD, Barreira TV, Kang M, Ainsworth BE. How many hours are enough? Accelerometer wear time may provide bias in daily activity estimates. J Phys Act Health. 2013;10(5):742-9.

36. Barreira TV, Hamilton MT, Craft LL, Gapstur SM, Siddique J, Zderic TW. Intra-individual and interindividual variability in daily sitting time and MVPA. J Sci Med Sport. 2015. Epub ahead of print.

37. Schram MT, Sep SJS, van der Kallen CJ, Dagnelie PC, Koster A, Schaper N, et al. The Maastricht Study: An extensive phenotyping study on determinants of type 2 diabetes, its complications and its comorbidities. Eur J Epidemiol. 2014;29(6):439-51.

38. Harris $T B$, Launer $L$, Eiriksdottir $G$, Kjartansson O, Jonsson P, Sigurdsson G, et al. Age, Gene/ Environment Susceptibility-Reykjavik Study: Multidisciplinary applied phenomics. Am J Epidemiol. 2007;165(9):1076-87.

39. Healy G, Wijndaele K, Dunstan D, Shaw J, Salmon J, Zimmet $P$, et al. Objectively measured sedentary time, physical activity, and metabolic risk. Diabetes Care. 2008;31(2):369-71.

40. Maher C, Olds T, Mire E, Katzmarzyk PT. Reconsidering the sedentary behaviour paradigm. PLoS One. 2014;9(1):e86403.

41. Schisterman EF, Cole SR, Platt RW.

Overadjustment bias and unnecessary adjustment in epidemiologic studies. Epidemiology. 2009;20(4):488-95.

42. Falconer $\mathrm{CL}$, Page AS, Andrews RC, Cooper AR. The potential impact of displacing sedentary time in adults with type 2 diabetes. Med Sci Sport Exerc. 2015;47(10):2070-5.

43. Hamer M, Stamatakis E, Steptoe A. Effects of substituting sedentary time with physical activity on metabolicrisk. Med Sci Sport Exerc. 2014;46(10): 1946-50. 
44. Healy GN, Winkler EAH, Owen N, Anuradha $S$, Dunstan DW. Replacing sitting time with standing or stepping : Associations with cardio-metabolic risk biomarkers. Eur Heart J. $2015 ; 14(36): 2643-9$.

45. Healy $\mathrm{GN}$, Winkler $\mathrm{EAH}$, Brakenridge $\mathrm{CL}$, Reeves MM, Eakin EG. Accelerometer-derived sedentary and physical activity time in overweight/obese adults with type 2 diabetes: Cross-sectional associations with cardiometabolic biomarkers. PLoS One. 2015;10:e0119140.

46. Buman MP, Winkler EAH, Kurka JM, Hekler EB, Baldwin CM, Owen N, et al. Reallocating time to sleep, sedentary behaviors, or active behaviors: Associations with cardiovascular disease risk biomarkers, NHANES 2005-2006. Am J Epidemiol. 2013;179(3):323-34.

47. Ekblom-Bak E, Ekblom $O$, Bergstro $m \mathrm{G}$, Bo riesson $M$. Isotemporal substitution of sedentary time by physical activity of different intensities and bout lengths, and its associations with metabolic risk. Eur J Prev Cardiol. 2015. Epub ahead of print.

48. Yates T, Henson J, Edwardson C, Dunstan $D$, Bodicoat DH, Khunti K, et al. Objectively measured sedentary time and associations with insulin sensitivity: Importance of reallocating sedentary time to physical activity. Prev Med. 2015;76:79-83

49. Benatti F, Ried-Larsen M. The effects of breaking up prolonged sitting time. Med Sci Sport Exerc. 2015;47(10):2053-61.

50. Hamilton MT, Hamilton DG, Zderic TW. Exercise physiology versus inactivity physiology: An essential concept for understanding lipoprotein lipase regulation. Exerc Sport Sci Rev. 2004;32(4):161-6.

51. Hamilton MT, Hamilton DG, Zderic TW. Sedentary Behavior as a mediator of type 2 diabetes. Diabetes and Physical Activity. 2014. p. 11-26.

52. Hamilton M, Hamilton D, Zderic T. Role of low energy expenditure and sitting in obesity, metabolic syndrome, type 2 diabetes, and cardiovascular disease. Diabetes. 2007;56:2655-67.

53. Duvivier BMFM, Schaper NC, Bremers MA van Crombrugge G, Menheere PPCA, Kars $M$, et al. Minimal intensity physical activity (standing and walking) of longer duration improves insulin action and plasma lipids more than shorter periods of moderate to vigorous exercise (cycling) in sedentary subjects when energy expenditure is comparable. PLoS One. 2013;8(2):e55542.

54. Thosar SS, Johnson BD, Johnston JD, Wallace JP. Sitting and endothelial dysfunction: The role of shear stress. Med Sci Monit. 2012;18(12):RA173-80.

55. Goessler K, Polito M, Cornelissen VA. Effect of exercise training on the renin-angiotensinaldosterone system in healthy individuals: A systematic review and meta-analysis. Hypertens Res. 2016;39(3):119-26.

56. Falconer $\mathrm{CL}$, Cooper $\mathrm{AR}$, Walhin JP, Thompson $D$, Page AS, Peters TJ, et al. Sedentary time and markers of inflammation in people with newly diagnosed type 2 diabetes. Nutr Metab Cardiovasc Dis. 2014;24(9):956-62.

57. Henson J, Yates T, Edwardson CL, Khunti K, Talbot D, Gray L, et al. Sedentary time and markers of chronic low-grade inflammation in a high risk population. PLoS One. 2013;8(10):e78350.

58. World Health Organization. Global status report on noncommunicable diseases 2014. 2014.

59. Matthews CE, Chen KY, Freedson PS, Buchowski MS, Beech M, Pate RR, et al. Amount of time spent in sedentary behaviors in the United States, 2003-2004. Am J Epidemiol. 2008;167(7):875-81.

60. Davis MG, Fox KR, Hillsdon M, Sharp DJ, Coulson JC, Thompson JL. Objectively measured physical activity in a diverse sample of older urban UK adults. Med Sci Sports Exerc. $2011 ; 43(4): 647-54$.

61. Arnardottir NY, Koster A, Van Domelen DR, Brychta RJ, Caserotti P, Eiriksdottir G, et al. Objective measurements of daily physical activity patterns and sedentary behaviour in older adults: Age, Gene/Environment Susceptibility-Reykjavik Study. Age Ageing. 2012;0:1-7.

62. Australian Government Department of Health Australia's Physical Activity and Sedentary Behaviour Guidelines For Adults (18-64 years). 2014. Available from: http://www.health.gov. au/internet/main/publishing.nsf/Content/phyactivity 
63. Department of Health Physical Activity Health Improvement and Protection. Start Active, Stay Active. Report. 2011 . Available from: https:// www.gov.uk/government/publications/startactive-stay-active-a-report-on-physical-activity-fromthe-four-home-countries-chief-medical-officers

64. Canadian Society of Exercise Physiology. Canadian Physical Activity and Sedentary Behaviour Guidelines. 2012. Available from: www.csep.ca/guidelines

65. Lee I-M, Shiroma EJ, Lobelo F, Puska P, Blair SN, Katzmarzyk PT. Effect of physical inactivity on major non-communicable diseases worldwide: An analysis of burden of disease and life expectancy. Lancet. 2012;380(9838):219-29.

66. US Department of Health and Human. 2008 Physical Activity Guidelines for Americans. 2008.

67. World Health Organization. Global recommendations on physical activity for health. 2010.

68. Warburton DE, Charlesworth S, Ivey A, Nettlefold L, Bredin SS. A systematic review of the evidence for Canada's Physical Activity Guidelines for adults. Int J Behav Nutr Phys Act. 2010;7:39. 



\title{
Summary
}

\section{Samenvatting}

\section{Valorisation addendum}

\author{
Dankwoord
}




\section{SUMMARY}

Since type 2 diabetes mellitus (T2DM), its complications and comorbidities pose an enormous burden on patients, healthcare systems, and society, the need for preventing T2DM is beyond dispute. A potential target for prevention strategies may be sedentary behaviour. Sedentary behaviour, such as watching TV, using the computer, or driving, has been identified as a risk factor for health during the early 2000s. Since then sedentary behaviour has been associated with cardio-metabolic outcomes in several studies. However, in most of these studies the measurement of sedentary behaviour was based on self-reporting methods that may have been subject to report and recall bias. Apart from that, as not all sedentary behaviour is bad (certain amounts are needed for rest and recovery), the pattern in which sedentary time is accumulated (e.g. many short sedentary periods versus one prolonged period) should be taken into consideration. Studies on health effects of sedentary behaviour patterns are limited. To overcome the limitations of self-reporting measures and expand knowledge on sedentary behaviour and its patterns, the studies in this dissertation have used accelerometry for the objective measurement of several sedentary behaviour constructs. Subsequently, their associations with cardiometabolic outcomes including adiposity, blood glucose and lipids levels, blood pressure, the metabolic syndrome, T2DM, aortic stiffness, and kidney function were examined. An overview of the main findings of each study (chapters 2-9) and concluding remarks are provided below.

\section{Chapter 2: Waking time in 24 hour accelerometry data}

In order to correctly determine the amount of sedentary time and physical activity executed during waking time, we developed an algorithm that automatically identifies wake and bed times on an individual level (i.e. different wake and bed times on each day for each participant). Subsequently, we demonstrated that this algorithm was highly associated with self-reported wake and bed times. So, using an accelerometer with a 24 hour/day wear time protocol was demonstrated to be feasible in large-scale epidemiological studies.

\section{Chapter 3: Movement during sedentary behaviour}

Sedentary behaviour is characterized by low levels of activity in the up-and-down (vertical) direction. However, during sedentary behaviour back-to-front and side-to-side movement is likely to occur, for example when doing desk work. We have demonstrated that a hip-worn 
tri-axial accelerometer indeed records significant amounts of movement in the back-to-front and side-to-side directions during sedentary activities such as playing cards or preparing food. Since this movement may be relevant for health, we recommend the use of tri-axial accelerometry data to examine associations of movement during sedentary activities and health outcomes.

\section{Chapter 4: Determinants of sedentary behaviour}

Since large amounts of sedentary behaviour have been associated with adverse metabolic and cardiovascular outcomes, insight into determinants of sedentary behaviour is warranted in order to prevent this behaviour and its adverse effects on health. Therefore, we have examined associations between a wide range of midlife determinants and objectively measured sedentary time in old age. The results show that in midlife, not being married, having lower educational level, living in poorer housing, being obese and having a heart disease were associated with considerably more sedentary time per day in old age. This information could be used to identify target groups for strategies aiming to prevent a highly sedentary lifestyle.

\section{Chapter 5: Movement during sedentary behaviour and cardio-metabolic outcomes}

Sedentary behaviour is characterized by no or low levels of activity in the up-and-down direction, but movement in the back-to-front and side-to-side directions during sedentary behaviour is likely to occur, for example when playing cards (chapter 3). Given the large amounts of time individuals spend being sedentary, examining associations of movement during sedentary behaviour ('active sedentariness') with health outcomes is relevant. We studied these associations and the results show that larger amounts of active sedentariness were associated with improved cardio-metabolic outcomes including a lower body mass index, a smaller waist circumference, improved levels of HDL cholesterol and triglycerides, and a lower odds for the metabolic syndrome. These findings suggest that even limited movement may affect physiologic mechanisms, which is an important finding for the development of sedentary behaviour guidelines aiming to prevent disease.

\section{Chapter 6: Reallocating sedentary time and cardio-metabolic outcomes}

Since large amounts of sedentary time have been associated with detrimental health effects, recommendations for reducing sedentary time have been developed. Reducing sedentary time inevitably results in more non-sedentary time during waking time. 
The health effects of these reductions may depend on the activity with which it is replaced, so we examined associations of a theoretical reallocation of sedentary time to standing or stepping with cardio-metabolic outcomes. The results show that replacing 30 minutes of sedentary time with 30 minutes of standing or stepping was associated with a lower body mass index, a smaller waist circumference, and improved cholesterol, triglycerides, glucose and insulin levels. In addition, similar reallocations were favourably associated with the metabolic syndrome and T2DM. These findings could be important for the general population, for those who cannot participate in high intensity activities, and for those who cannot meet the physical activity guidelines, because they suggest that small replacements of sedentary time may have favourable effects on health even if sedentary time was replaced by standing.

\section{Chapter 7: Sedentary behaviour, the metabolic syndrome and T2DM}

Given the high prevalence of T2DM, it is important to gain insight into the associations of sedentary behaviour with (risk factors for) T2DM in order to prevent it. Therefore, we conducted a study that examined associations of total amount and patterns of sedentary behaviour with the metabolic syndrome and T2DM. The results show that larger amounts of sedentary time were associated with increased odds for the metabolic syndrome as well as T2DM. The pattern in which sedentary time was accumulated was weakly associated with the presence of the metabolic syndrome. These results suggest that sedentary behaviour may play a significant role in the development of T2DM. Therefore, consideration should be given to including strategies aiming to reduce the amount of sedentary time in diabetes prevention programmes.

\section{Chapter 8: Sedentary behaviour and aortic stiffness}

Aortic stiffness has been related to cardiovascular events and mortality. In order to prevent cardiovascular disease, prevention programs should focus on modifiable lifestyle factors that relate to aortic stiffness. Since sedentary behaviour may be such a factor, we examined associations of total amount and patterns of sedentary behaviour with aortic stiffness. We found no significant associations of both amount and patterns of sedentary behaviour with aortic stiffness, possibly due to relatively homogeneous study population. Since this study was one of the first in which amount and patterns of objectively measured sedentary behaviour were examined in relation to aortic stiffness, more accelerometry studies are needed in order to confirm and compare our results. 


\section{Chapter 9: Sedentary behaviour and kidney function}

A reduced estimated glomerular filtration rate (eGFR) and albuminuria, which together define chronic kidney disease, are associated with end-stage renal disease and cardiovascular disease. To prevent these diseases, it is important to identify modifiable risk factors for reduced eGFR and albuminuria, for example sedentary behaviour. Therefore, we examined associations of total amount and patterns of sedentary behaviour with eGFR and albuminuria. The results show adverse associations of total amount of sedentary time with both eGFR and albuminuria. In addition, associations between constructs of sedentary behaviour patterns and eGFR were demonstrated. Given these results, reducing the amount of sedentary time may be a relevant target in programs aiming to prevent a reduced eGFR and albuminuria.

\section{Concluding remarks}

The studies in this dissertation have consistently shown that large amounts of sedentary time are associated with detrimental cardio-metabolic outcomes. Since the majority of individuals has shown to spend on average more than half of the waking day in sedentary positions, the potential impact of our findings on a population level may be relevant. Therefore, recommendations on reducing sedentary time to prevent its adverse effects on health are needed. For the development of effective recommendations and sedentary behaviour guidelines, experimental studies, dose-response studies, and feasibility studies are warranted. In addition, longitudinal studies should be conducted to advance knowledge on causality and physiological studies are needed to unravel the mechanisms through which sedentary behaviour affects health. 


\section{SAMENVATTING}

Type 2 diabetes, ook wel suikerziekte genoemd, is een chronische stofwisselingsziekte die gekenmerkt wordt door verhoogde glucosewaardes in het bloed. Op de lange termijn kunnen deze verhoogde glucosewaardes leiden tot chronische schade aan nieren (nefropathie), zenuwen (neuropathie) en ogen (retinopathie). Daarnaast gaat type 2 diabetes vaak gepaard met ongunstige cardio-metabole uitkomsten zoals overgewicht, hoge bloeddruk en afwijkende waardes van cholesterol en triglyceriden in het bloed, waardoor er een veel grotere kans is op cardiovasculaire ziektes.

Naar schatting zijn er wereldwijd 415 miljoen mensen die lijden aan een vorm van diabetes en naar verwachting zullen dit er 642 miljoen zijn in 2040 (10\% van de volwassen wereldbevolking). Vanwege de chronische complicaties, comorbiditeit en hoge prevalentie zijn de gevolgen van type 2 diabetes groot voor de patiënt, het zorgsysteem en de samenleving. Preventie van type 2 diabetes is daarom erg belangrijk.

Een mogelijk richtpunt in preventiestrategieën is sedentair gedrag. Sedentair gedrag wordt gekenmerkt door activiteiten waarbij het energieverbruik niet of nauwelijks boven het energieverbruik in rust uitkomt, zoals televisie kijken, computeren of autorijden. Uit verschillende studies is gebleken dat het vertonen van veel sedentair gedrag samenhangt met onder andere een hoog gewicht, verhoogde glucosewaardes, het hebben van metabool syndroom en het hebben van type 2 diabetes. In deze studies werd sedentair gedrag gemeten met behulp van vragenlijsten die (erg) gevoelig zijn voor onbetrouwbare metingen. Daarnaast is er in voorgaande studies niet gekeken naar patronen van sedentair gedrag. Hiermee wordt de manier bedoeld waarop sedentaire tijd over de dag is verspreid, bijvoorbeeld weinig sedentaire periodes van lange duur of veel sedentaire periodes van korte duur. Het is relevant om ook naar deze patronen te kijken, omdat niet alle sedentaire periodes nadelig zijn voor de gezondheid en zelfs een zekere hoeveelheid sedentair gedrag noodzakelijk is voor rust en herstel.

Vanwege de beperkingen van vragenlijsten die ervoor zorgen dat sedentair gedrag niet accuraat gemeten wordt en het ontbreken van onderzoek naar sedentaire patronen, hebben de studies in dit proefschrift gebruikt gemaakt van accelerometrie. Accelerometers zijn kleine draagbare apparaatjes die zowel frequentie, als intensiteit en duur van alle dagelijkse activiteiten meten. Hiermee kan op een objectieve manier gemeten worden hoeveel tijd sedentaire tijd is en wat het patroon van het sedentaire gedrag is. In dit proefschrift is onderzocht hoe zowel sedentair gedrag als patronen van sedentair gedrag 
samenhangen met cardio-metabole vitkomsten (zoals gewicht, bloeddruk en cholesterol), het metabool syndroom, type 2 diabetes, vaatstijfheid en nierfunctie. Hieronder wordt een overzicht gegeven van de belangrijkste bevindingen en conclusies.

\section{Hoofdstuk 2: Wakkertijd in 24 uurs accelerometrie data}

Om de hoeveelheid sedentaire tijd te bepalen, is het belangrijk om te bepalen wanneer iemand wakker is geweest. Dit voorkomt dat slapen als sedentair gedrag wordt geclassificeerd of andersom. Een methode om de wakkere tijd automatisch te bepalen bestond nog niet. We hebben daarom een algoritme ontwikkeld dat voor elke proefpersoon, op elke dag de wakkere tijd bepaalt. Vervolgens hebben we dit algoritme getest en aangetoond dat de tijden die door het algoritme bepaald werden vergelijkbaar waren met de zelfgerapporteerde tijden van proefpersonen. Het algoritme kan daarom gebruikt worden om op een accurate wijze wakkertijd te identificeren, waardoor het haalbaar is geworden om grootschalige epidemiologische studies uit te voeren waarin accelerometers 24 uur per dag gedragen worden.

\section{Hoofdstuk 3: Bewegen tijdens sedentair gedrag}

Sedentair gedrag wordt gekenmerkt door weinig activiteit in de verticale richting (onder-boven). Echter, tijdens sedentair gedrag beweegt het bovenlichaam waarschijnlijk wel van links naar rechts (zijwaarts) of van voor naar achter (voorwaarts), bijvoorbeeld tijdens het lezen van de krant. Het was vooralsnog onbekend of een accelerometer die wordt gedragen op de heup, deze zij- en voorwaartse beweging kan meten. Om dit uit te zoeken hebben wij een experiment uitgevoerd. Daaruit bleek dat een accelerometer die wordt gedragen op de heup inderdaad voor- en zijwaartse beweging kan meten tijdens sedentaire activiteiten zoals het lezen van de krant en het spelen van yahtzee. Het is mogelijk dat deze beweging relevant is voor de (cardio-metabole) gezondheid, dus studies naar de samenhang tussen bewegen tijdens sedentair gedrag en gezondheid worden aanbevolen.

\section{Hoofdstuk 4: Determinanten van sedentair gedrag}

Veel sedentair gedrag hangt samen met een slechtere cardio-metabole gezondheid. Om nadelige effecten van sedentair gedrag op de gezondheid te voorkomen, is het belangrijk om te bepalen welke factoren bijdragen aan sedentair gedrag. Daarom hebben wii onderzocht welke factoren op middelbare leeftijd (gemiddeld 48 jaar) samenhangen met 
sedentair gedrag op hoge leeftijd (gemiddeld 80 jaar). De resultaten laten zien dat de aanwezigheid van (één van de) volgende factoren op middelbare leeftijd samenhangt met maximaal 39 minuten meer sedentaire tijd op hoge leeftijd: ongehuwd zijn, een laag opleidingsniveau hebben, wonen in minder goede huisvesting, het hebben van obesitas en het hebben van een hartziekte. Deze informatie kan gebruikt worden om risicogroepen voor een overwegend sedentaire leefstijl vroegtijdig te identificeren.

\section{Hoofdstuk 5: Bewegen tijdens sedentair gedrag en cardio-metabole vitkomsten}

Sedentair gedrag wordt gekenmerkt door weinig activiteit in de verticale richting (onder-boven), maar zij- en voorwaartse beweging tijdens sedentaire activiteiten is wel waarschijnlijk, bijvoorbeeld bij het spelen van yahtzee (hoofdstuk 3). Het onderzoeken van de samenhang tussen deze bewegingen en gezondheid is relevant, omdat de meerderheid van de bevolking het grootste gedeelte van de tijd sedentair doorbrengt. Wii hebben daarom associaties tussen bewegen tijdens sedentair gedrag en cardio-metabole vitkomsten onderzocht. Veel sedentaire tijd met beweging bleek samen te hangen met een lagere 'body mass index' (BMI), een kleinere buikomvang, betere waardes van HDL-cholesterol en triglyceriden in het bloed en een lagere kans op het hebben van het metabool syndroom. Deze bevindingen suggereren dat zelfs minimale beweging invloed heeft op fysiologische mechanismen in het lichaam en dat is belangrijke informatie voor de ontwikkeling van richtlijnen voor sedentair gedrag.

\section{Hoofdstuk 6: Vervangen van sedentair gedrag door staan of lopen en cardio-metabole vitkomsten}

Sedentair gedrag hangt samen met gezondheid en daarom zijn er richtlijnen ontwikkeld die gericht zijn op het verminderen van sedentaire tijd. Het verminderen van sedentaire tijd tijdens de wakkere tijd leidt onherroepelijk tot meer niet-sedentaire tijd. De effecten van het verminderen van sedentaire tijd op de gezondheid, hangen daarom mogelijk af van het gedrag waarmee sedentair gedrag wordt vervangen. Om deze effecten te onderzoeken hebben wii gekeken naar veranderingen in cardio-metabole vitkomsten wanneer sedentair gedrag vervangen wordt door staan of lopen. Het vervangen van 30 minuten sedentair gedrag door 30 minuten staan of lopen was geassocieerd met een lagere 'body mass index' (BMI), een kleinere buikomvang en betere waardes van cholesterol, triglyceriden, glucose en insuline in het bloed. Daarnaast bleek het vervangen samen te hangen met een lagere kans op het hebben van het metabool syndroom en 
type 2 diabetes. Deze bevindingen zijn belangrijk voor de algemene bevolking en in het bijzonder voor hen die niet aan de richtlijnen voor bewegen kunnen voldoen (ouderen, zieken), omdat het vervangen van een korte sedentaire periode door lopen en zelfs door staan een gunstig effect kan hebben op gezondheid.

\section{Hoofdstuk 7: Sedentair gedrag, het metabool syndroom en type 2 diabetes}

Type 2 diabetes is een veelvoorkomende ziekte. Om een verdere toename van type 2 diabetes te voorkomen is het belangrijk om inzicht te krijgen in de samenhang tussen sedentair gedrag en (risicofactoren van) type 2 diabetes. Daarom hebben wij associaties tussen enerzijds sedentaire tijd en sedentaire patronen en anderzijds het metabool syndroom en type 2 diabetes onderzocht. De resultaten laten zien dat elk extra uur sedentaire tijd geassocieerd was met een $39 \%$ grotere kans op het hebben van het metabool syndroom en een $22 \%$ grotere kans op het hebben van type 2 diabetes. Sedentaire patronen waren alleen zwak geassocieerd met het hebben van het metabool syndroom. Deze resultaten suggereren dat sedentair gedrag een belangrijke rol speelt in het ontstaan van type 2 diabetes. Het verminderen van sedentair gedrag zou daarom onderdeel moeten worden van preventieprogramma's die gericht zijn op het voorkomen van type 2 diabetes.

\section{Hoofdstuk 8: Sedentair gedrag en vaatstijfheid}

Vaatstijfheid verwijst naar de mate waarin de wanden van de (grote) slagaders elastisch zijn. Hoe minder elastisch, hoe stijver de slagaders. Een verhoogde vaatstijfheid hangt samen met cardiovasculaire ziektes en mortaliteit. Om die te voorkomen zouden preventieprogramma's gericht moeten zijn op factoren die samenhangen met verhoogde vaatstijfheid en die te veranderen zijn. Sedentair gedrag zou een dergelijke factor kunnen zijn en daarom hebben we onderzocht wat de samenhang is tussen enerzijds sedentaire tijd en sedentaire patronen en anderzijds vaatstijfheid. Er werden geen statistisch significante associaties gevonden tussen 1) sedentaire tijd en vaatstijfheid en 2) sedentaire patronen en vaatstijfheid. Dit werd mogelijk veroorzaakt door de homogene studiepopulatie. Er zijn daarom meer accelerometriestudies nodig die de samenhang van sedentair gedrag, sedentaire patronen met vaatstijfheid onderzoeken. 


\section{Hoofdstuk 9: Sedentair gedrag, nierfunctie en -schade}

Een verminderde nierfunctie en nierschade hangen samen met chronische nierziekten en cardiovasculaire ziekten. Om deze ziektes te voorkomen zouden preventieprogramma's gericht moeten zijn op factoren die te veranderen zijn en die samenhangen met een verminderde nierfunctie en nierschade. Sedentair gedrag zou een dergelijke factor kunnen zijn en daarom hebben we onderzocht wat de samenhang is tussen enerzijds sedentaire tijd en sedentaire patronen en anderzijds nierfunctie en nierschade. Het hebben van meer sedentaire tijd bleek samen te hangen met een verminderde nierfunctie en tekenen van nierschade. Sedentaire patronen waren alleen geassocieerd met een verminderde nierfunctie. Het reduceren van sedentaire tijd zou daarom opgenomen moeten worden in preventieprogramma's die gericht zijn op het voorkomen van een verminderde nierfunctie en nierschade.

\section{Conclusies}

De studies in dit proefschrift hebben aangetoond dat grote hoeveelheden sedentair gedrag samenhangen met nadelige cardio-metabole vitkomsten, het metabool syndroom en type 2 diabetes. De gevolgen hiervan zouden op bevolkingsniveau aanzienlijk kunnen zijn, omdat het merendeel van de bevolking het grootste gedeelte van de dag sedentair doorbrengt. Het is daarom belangrijk om effectieve richtlijnen voor het verminderen van de hoeveelheid sedentair gedrag te ontwikkelen. Daarvoor is het van belang om door middel van experimentele studies, dosis-respons studies en haalbaarheidsstudies inzicht te krijgen in de mate waarin sedentair gedrag verminderd moet worden en de manier waarop dat bereikt kan worden. Daarnaast zijn lange-termijnstudies nodig om oorzaak en gevolg vast te stellen en kunnen fysiologische studies inzicht geven in de mechanismen die ertoe leiden dat sedentair gedrag de gezondheid beïnvloedt. 


\section{VALORISATION ADDENDUM}

An important aspect of research is to ensure its results will impact society by making it suitable or available for social or economic use and by making it suitable for translation into products, services, processes or new commercial activities. This valorisation addendum will describe how our results impact society.

Type 2 diabetes mellitus (T2DM) is one of the most prevalent diseases worldwide. With its complications and comorbidities T2DM imposes an enormous burden on not only patients, but on healthcare systems and society as well. So the need to prevent T2DM, its complications and comorbidities is beyond dispute. In order to do this, it should be examined which factors play a role in the development of T2DM, its complications and comorbidities.

Further, such factors should be modifiable and should occur frequently.

The studies in this dissertation have consistently shown that sedentary behaviour (such as sitting, using the computer or driving) was associated with cardio-metabolic health. For example, large amounts of sedentary behaviour were strongly associated with a larger waist circumference, a higher body mass index, dyslipidaemia, the metabolic syndrome, and T2DM. So, sedentary behaviour seems to be an important risk factor for health and seems to be a relevant factor in the development of T2DM, its complications and comorbidities. In addition, sedentary behaviour is a factor which can be modified and which occurs frequently as the majority of individuals has been shown to spend on average more than half of the waking day in sedentary positions. Given this and the results presented in this dissertation, sufficient evidence is provided for the development of sedentary behaviour guidelines by governmental health departments, public health organizations or scientific institutions. These guidelines should focus on strategies to reduce the daily amounts of sedentary behaviour. In the United Kingdom, Canada and Australia, such guidelines already have been introduced. For clinical practice the method to measure sedentary behaviour as well as the results as described in this dissertation form a first step towards personalized care. Accelerometers could be used by general practitioners to identify individuals with a highly sedentary lifestyle. Subsequently, this could be used to tailor interventions at an individual level.

In addition to its applicability in public health settings and clinical practice, the results of this dissertation give rise to intervene in the public domain. The built environment should be designed in a way it discourages sedentary behaviour and facilitates physical activity, 
for example by providing green and public spaces in neighbourhood and schools and increasing the level of walkability in cities. Further, it may also encourage employers to reduce the amount of sedentary behaviour of their employees by providing stand-desks and organizing walking meetings. This may contribute not only to the health status of employees, but may improve work performance and productivity as well. Finally, this dissertation may have convinced individuals to reduce their sedentary behaviour, which can be achieved by simple, personal interventions such as standing up during commercial breaks while watching TV, standing or walking during phone calls, or making a walk during lunch break or after dinner.

\section{Future research}

In order to reduce sedentary behaviour on a population as well as an individual level, recommendations should be developed that specify the amount of time and the type of activity with which sedentary time should be replaced. In this dissertation it was demonstrated that, theoretically, replacement of small amounts of sedentary time (i.e. 30 minutes) with non-sedentary time (i.e. standing and stepping) was associated with beneficial metabolic outcomes, the metabolic syndrome and T2DM. This information provides directions for future dose-response studies that should be conducted in order to obtain insight into harmful amounts of sedentary time. Our results also provide directions for experimental and intervention studies that should be conducted to examine the effects of replacing sedentary time with non-sedentary time on health outcomes.

In addition, the results presented in this dissertation strongly suggest that sedentary behaviour is an important risk factor for health. However, the studies were cross-sectional in nature which hampers the determination of causal relationships. Therefore, longitudinal research in which sedentary behaviour and health outcomes will repeatedly be measured over time are needed. Ideally, in such studies sedentary behaviour will be measured objectively during 24 hour per day on multiple days in a large study population. With the algorithm we have developed, it has become feasible to conduct such studies. 


\section{$\underline{\text { DANKWOORD }}$}

Dit proefschrift was er niet geweest zonder de hulp van velen. $\mathrm{Zij}$ die op enigerlei wijze geholpen hebben, familie, vrienden, teamgenoten in binnen- en buitenland, collega's, coauteurs en de leden van de beoordelingscommissie, wil ik hierbij dan ook hartelijk danken. Zes van hen dank ik in het bijzonder:

Hans, dank voor de altijd razendsnelle en positieve feedback die je hebt gegeven op mijn manuscripten, abstracts, posters en presentaties. Daarnaast ben je alle jaren heel positief en enthousiast geweest over mijn project, soms nog enthousiaster dan ik, en dat werkte aanstekelijk. Hartelijk dank daarvoor.

Annemarie, vanaf dag 1 heb je me vrijgelaten om de dingen op mijn eigen manier te doen en dat ik waardeer ik zeer. Ook was je altijd heel enthousiast en positief, gaf je snelle en waardevolle feedback en kon ik op elk moment bij je binnenlopen voor allerhande vragen. Veel dank daarvoor.
Coen, in de afgelopen 4 jaar heb je me naar allerlei cursussen en congressen gestuurd zodat ik me verder kon ontwikkelen en daar ben ik je dankbaar voor. Waar ik je daarnaast graag voor wil bedanken is de tijd die je hebt genomen om naar mijn verhalen te luisteren, je hulp en je support.

Jeroen, vanaf het begin zijn we samen opgetrokken, wat ertoe geleid heeft dat je de enige bent die ál mijn teksten gelezen heeft. Maar niet alleen met mijn teksten, ook met mijn frustraties, geklaag en vragen kon ik bij je terecht en daar ben ik je zeer dankbaar voor. Ik heb lang met je samen mogen werken, waarbij ik altijd op je kon rekenen en vertrouwen en wat bovendien altijd leuk was. Heel veel dank!

Jeroen, wat heb ik een ongelofelijk geluk gehad dat jii de andere 'activPAL aio' bent. Daardoor heb ik veel met je samen mogen werken, wat niet alleen heel prettig was, maar ook heel erg leuk. En dankzii jou werden de cursussen en congressen waar we samen naar toe mochten fantastische dagen die ik nooit zal vergeten. Ook kon ik altijd bij je terecht, altijd op je rekenen en je blind vertrouwen, en daar ben ik je ontzettend dankbaar voor. Je bent mijn allerbeste vriend, en die vriendschap is het mooiste en beste dat 4 jaar Maastricht me heeft opgeleverd. 
Huib, hoewel je er helemaal niet op zat te wachten dat ik naar Maastricht zou vertrekken, heb je altijd achter mijn keuze gestaan en ben je me zelfs achterna gekomen. Daar ben ik ontzettend gelukkig mee en dat heeft ook zeker positief bijgedragen aan de inhoud en kwaliteit van dit proefschrift. Wie anders kan me oneindig vaak en met engelengeduld iets over gemiddeldes vitleggen? Gelukkig kon ik ook altijd rekenen op je scherpe kritiek, je sterke taal- en rekenkundige inzicht en je onvoorwaardelijke steun. Tot en met de laatste punt en komma sta je achter mij en mijn keuzes en dat is onmisbaar. DANK! 
The Maastricht Study is an ongoing observational, population-based cohort study that focuses on the aetiology, pathophysiology, complications, and comorbidities of type 2 diabetes mellitus and which is characterised by an extensive phenotyping approach.

In this dissertation data is used from the first 3,451 participants who completed the baseline survey between November 2010 and September 2013. It was used to examine associations of sedentary behaviour with cardio-metabolic health, including the metabolic syndrome, type 2 diabetes, vascular function and kidney function. 